\title{
EFFECT OF VEHICLE CONNECTIVITY ON THE SAFETY PERFORMANCE OF FREEWAY ACCELERATION SPEED CHANGE LANES
}

\author{
by \\ Naga Haneesha Kanteti \\ B.E., Andhra University, India
}

A thesis submitted to the Faculty of Graduate and Postdoctoral Affairs in partial fulfillment of the requirements for the degree of

\author{
Master of Applied Science \\ in
}

Civil Engineering

Carleton University

Ottawa, Ontario

(C) 2019, Naga Haneesha Kanteti 


\begin{abstract}
One of the major concerns at the freeway entrance ramps is merging conflicts at the entrance ramp areas, which are highly prone to crashes according to past research. Ample length of speed change lanes (SCL) could provide enough time to SCL drivers, to search for a suitable gap and to adjust their speed to perform the merging action. Connected vehicle technology (CVT) enables the collection of real time and detailed vehicular data with the help of wireless communication sensors.

In this research, two merging maneuvers were examined by considering the capabilities of connected vehicles (CVs) to make the merging process more comfortable, and thus safer. Probability of non-compliance (PNC) which is a measure of probability of SCL driver's uncomfortable merging was calculated considering the gap searching and acceleration behaviour in a connected vehicle environment. Variations in the PNC values when compared with the regular vehicles were examined and safety benefits of CVs in terms of reducing the collision frequency on entrance ramps were examined. Sensitivity analysis was performed to investigate the effect of change in each input parameter on the output PNC measures.
\end{abstract}




\section{ACKNOWLEDGEMENTS}

I would first like to express sincere thanks to my thesis advisor Dr. Yasser Hassan, for his valuable guidance and continuous support throughout the completion of this research. The door to Prof. Yasser Hassan office was always open whenever I ran into a problem or had a question about my research or writing. His patience, valuable suggestions, and time are highly appreciable.

I would also like to thank Dr. Bashar Dhahir for his time and valuable feedback on my thesis. I am grateful to Louis Vachon (MTO - Communications Centre Supervisor, Ottawa) for his efforts to provide me the traffic volume data. I would like to express my gratitude to Ryan Taylor (Research Computing Services, Carleton University) for providing me access to the virtual server and assisting me in submitting the programming jobs. Access to virtual server from Prof. Siva Sivathayalan (Carleton University) was also acknowledged.

I would like to express my gratitude to Mr. Cuckoo Kochar for offering me the Kochar Family scholarship and for encouraging me with his inspiring words throughout my research.

I am always grateful to my godfather for his blessings through out my academic journey.

I would like to thank my dearest friends Luckson Kamisa, Keeshia Wee Eng for spending their valuable time on reading my thesis and providing the feedback. I would also like to thank Sandeep Kumar Jampani for his constant support and encouragement. All the 
encouragement and continuous support from each friend and well wisher of mine was greatly acknowledged.

I must express my very profound gratitude to my husband, my in-laws, my brother-in-law, and to my parents for providing me with unfailing support and continuous encouragement throughout my years of study and through the process of researching and writing this thesis. This accomplishment would not have been possible without them.

Finally, I would like to dedicate this thesis to my belated father-in-law, who had believed in me and inspired me all the time to become successful in life.

Naga Haneesha Kanteti 


\section{Table of Contents}

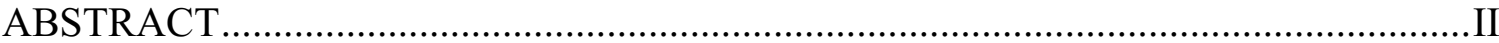

ACKNOWLEDGEMENTS ..................................................................................... III

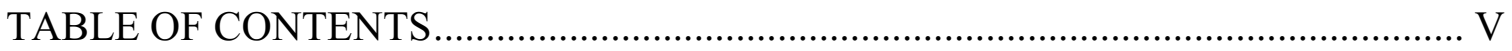

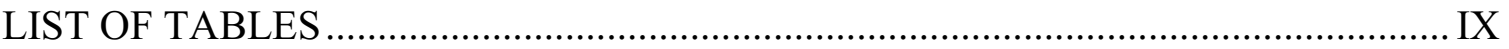

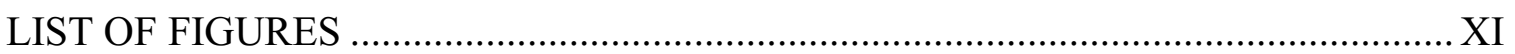

LIST OF ACRONYMS .................................................................................

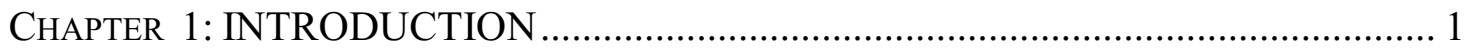

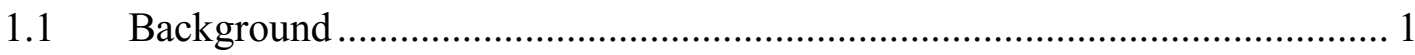

$1.2 \quad$ Problem Definition................................................................................. 3

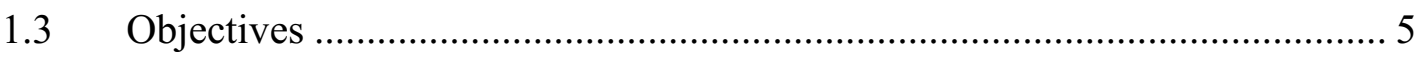

1.4 Scope

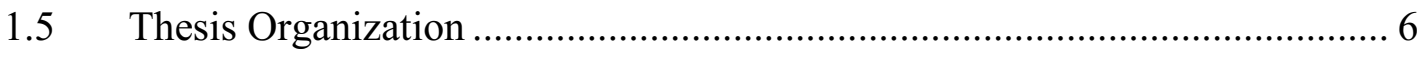

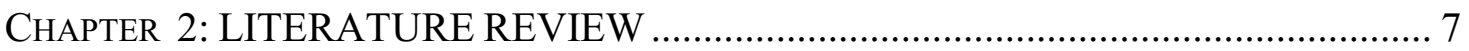

2.1 Review of SCL Design from the Guides AASHTO (2011) and TAC (2017) 7

2.1.1 Definitions of Design Elements ............................................................. 7

2.1.2 Design Guidelines for Acceleration Lane Length .................................. 14

2.2 Previous Research on Acceleration Length Design ....................................... 16

2.3 Reliability Concept in Geometric Design ................................................... 19

2.4 Previous Research on Freeway Merging Behaviour..................................... 22

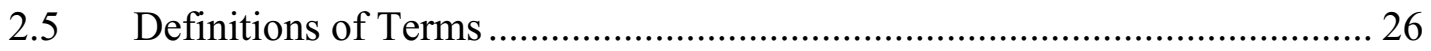




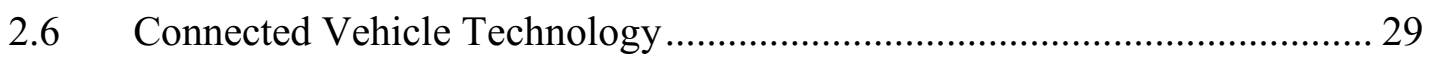

2.6.1 Benefits and Applications of Connected Vehicles.................................. 31

2.6.2 Connected Vehicle Deployment Program .................................................. 35

2.6.3 Existing Research on Freeway Merging in Connected Vehicle

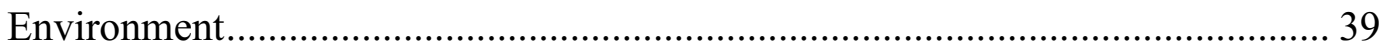

CHAPTER 3: DATA COLLECTION AND PRELIMINARY ANALYSIS .................... 43

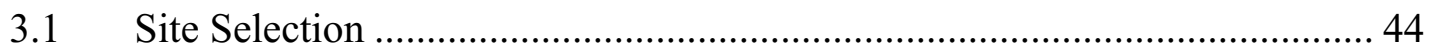

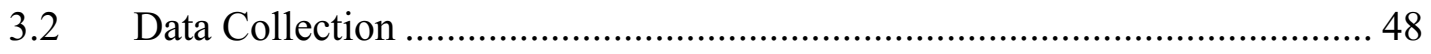

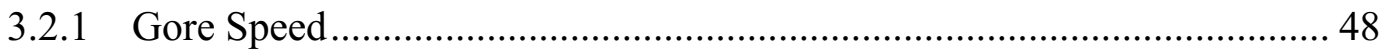

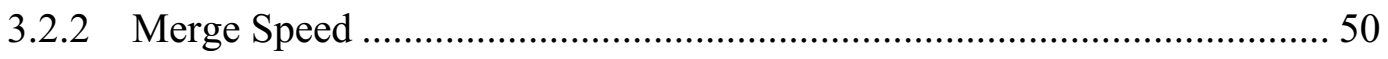

3.2.3 FRL Vehicle Speed ……………………........................................... 51

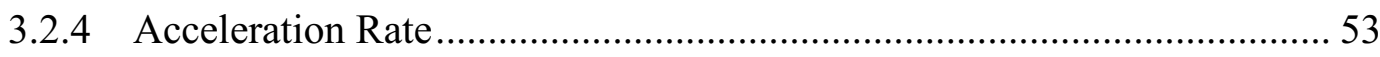

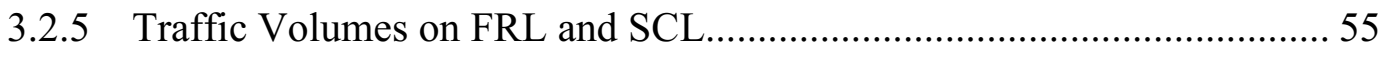

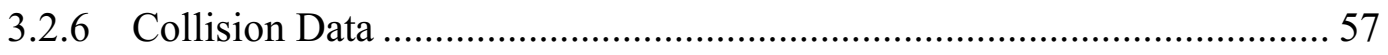

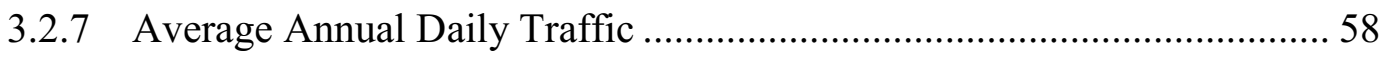

3.3 Traffic Volume on F2L and Lane Ratio ................................................... 59

3.4 Normality Tests for Parameters ................................................................... 62

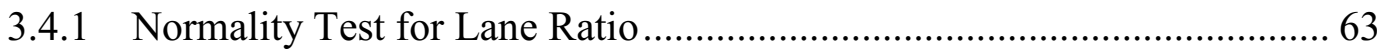

3.4.2 Normality Tests for Percentage of HV on F2L......................................... 63

CHAPTER 4: REGRESSION ANALYSIS OF SPEED PARAMETERS........................ 64

4.1 Model for $85^{\text {th }}$ Percentile Gore Speed........................................................... 64

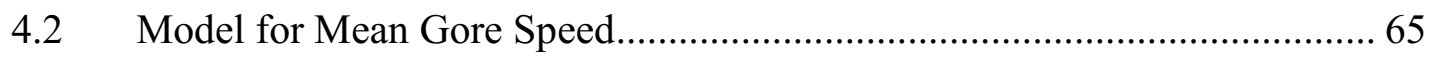

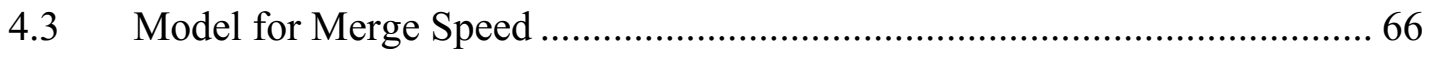




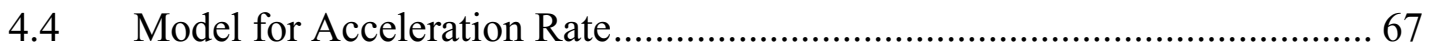

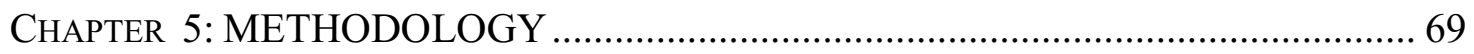

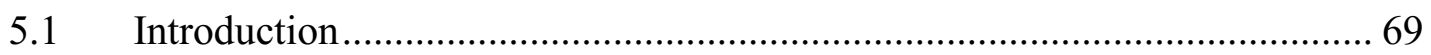

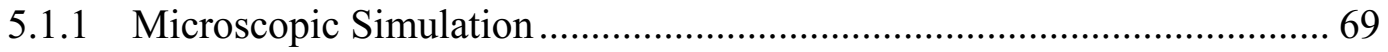

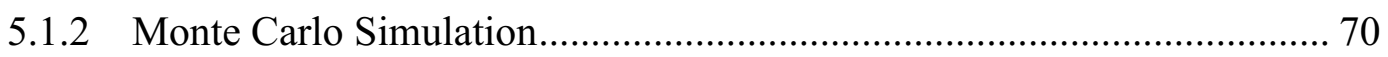

5.2 Modeling the Merging Process ………………........................................ 70

5.2.1 Framework of the Simulation Models ................................................... 75

5.2.2 SCL Vehicle Characteristics and Input Parameters ................................. 76

5.2.3 Freeway Vehicle Characteristics and Input Parameters............................ 79

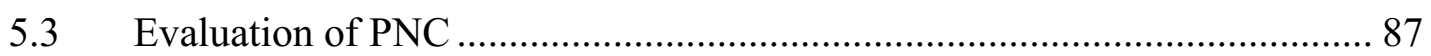

5.3.1 Calculation of Available Gaps in Freeway Lanes.................................... 87

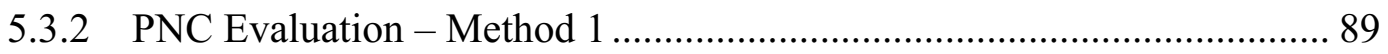

5.3.3 PNC Evaluation - Method 2 ……………......................................... 92

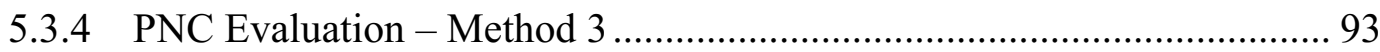

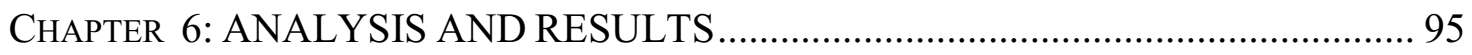

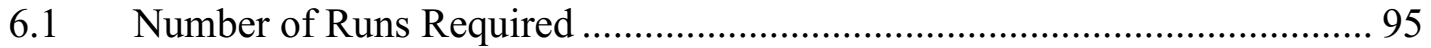

6.2 PNC at the Study SCL Locations.............................................................. 98

6.3 Relationship Between PNC and Collision Frequency ……........................ 103

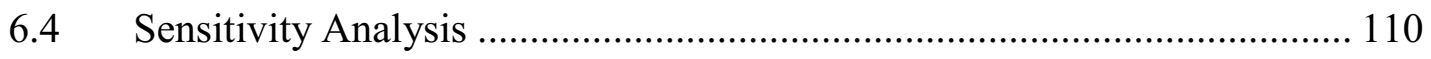

6.4.1 Effect of Change in Gore Speed ........................................................ 111

6.4.2 Effect of Change in FRL Speed ........................................................ 113

6.4.3 Effect of Change in F2L Speed.......................................................... 116

6.4.4 Effect of Change in Percentage of HV at FRL .................................... 118 
6.4.5 Effect of Change in Percentage of HV at F2L ................................... 120

6.4.6 Effect of Change in FRL Traffic Volume........................................... 122

6.4.7 Effect of Change in Lane Ratio ..................................................... 124

6.4.8 Effect of Change in SCL Length .................................................. 126

6.4.9 Effect of Change in SCL Traffic Volume......................................... 128

6.5 Application of Proposed Methodologies to the Design Guides................. 130

CHAPTER 7: CONCLUSIONS ........................................................................ 135

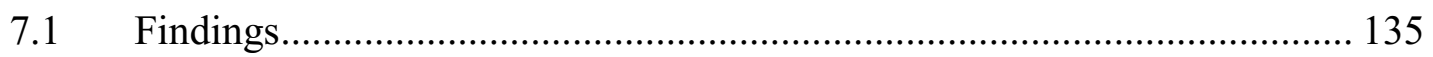

7.2 Further Research Recommendations ..................................................... 136

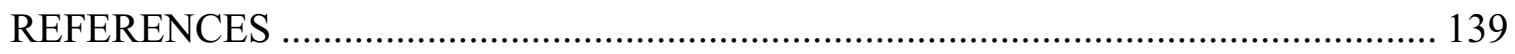

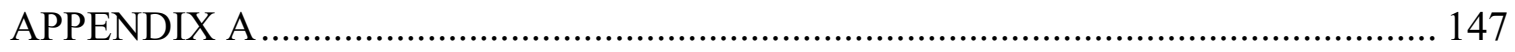

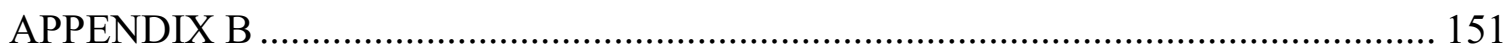




\section{List of Tables}

Table 2.1: Ramp Design Speed (TAC, 2017) .......................................................... 10

Table 2.2: Design Length for Acceleration Lane (TAC, 2017)........................................ 14

Table 2.3: Minimum Acceleration Lengths for Entrance Terminals (AASHTO, 2011).. 15

Table 2.4: Potential Benefits of CV Safety Applications (Hill et al., 2013)..................... 32

Table 3.1: List of SCL Sites (Ahammed, 2005). .............................................................. 46

Table 3.2: Geometry of the SCL Sites (Ahammed, 2005).............................................. 47

Table 3.3: Gore Speeds at Study SCL Sites.................................................................. 49

Table 3.4: Merge Speeds at Study SCL Sites. .............................................................. 50

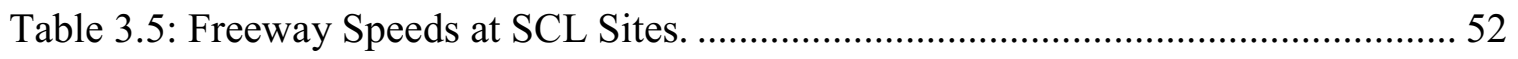

Table 3.6: Acceleration Rates at SCL Sites.................................................................. 54

Table 3.7: Traffic Volumes on FRL and SCL. ............................................................ 55

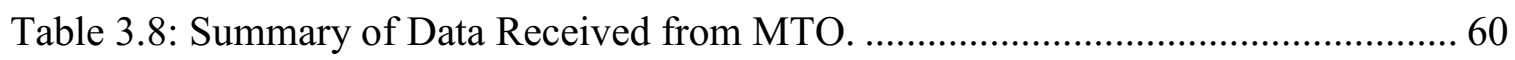

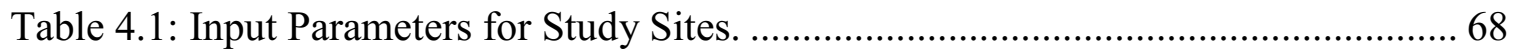

Table 6.1: Mean PNC Values for Different Number of Model Runs................................ 97

Table 6.2: PNC Values at the Study SCL Sites............................................................ 99

Table 6.3: Percentage Ranges of PNC Values - Method 1.......................................... 100

Table 6.4: Percentage Ranges of PNC Values - Method 2............................................. 101

Table 6.5: Percentage Ranges of PNC Values - Method 3.......................................... 102

Table 6.6: Collision Prediction Models with AADT and SCL Length........................... 104

Table 6.7: Collision Prediction Models with AADT, SCL Length and Mean PNC....... 106

Table 6.8: Predicted Collision Frequencies from the CPM........................................... 109

Table 6.9: Input Parameters for Sensitivity Analysis. ................................................ 110 
Table 6.10: Mean PNC and Change in Mean PNC for the Change in Gore Speed....... 111

Table 6.11: Mean PNC and Change in Mean PNC for the Change in Gore Speed........ 113

Table 6.12: Mean PNC and Change in Mean PNC for the Change in FRL Speed. ....... 114

Table 6.13: Mean PNC and Change in Mean PNC for the Change in F2L Speed........ 116

Table 6.14: Mean PNC and Change in Mean PNC with Change in Percentage of HV on

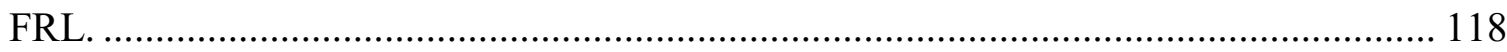

Table 6.15: Mean PNC and change in mean PNC with change in percentage of HV on

F2L

Table 6.16: Mean PNC and Change in Mean PNC for the Change in FRL Traffic

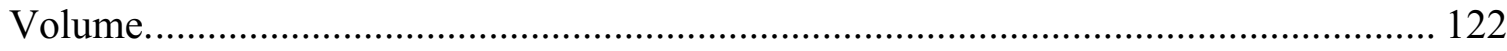

Table 6.17: Mean PNC and Change in Mean PNC for the Change in Lane Ratio........ 124

Table 6.18: Mean PNC and Change in Mean PNC with the Change in SCL Length. ... 126

Table 6.19: Mean PNC and Change in Mean PNC with the Change in SCL Traffic

Volume. 128

Table 6.20: PNC Values for the Recommended SCL Lengths by AASHTO (2011) Guide. 132

Table 6.21: PNC Values for the Recommended SCL Lengths by TAC (2017) Guide. . 133 


\section{List of Figures}

Figure 2.1: Basic Interchange Configurations (Figure 10-1, AASHTO, 2011)................. 8

Figure 2.2: General Types of Interchange Ramps (AASHTO, 2011). .............................. 9

Figure 2.3: Typical Entrance Gore Area (AASHTO, 2011)........................................... 11

Figure 2.4: Taper and Parallel Type Entrance Ramps (AASHTO, 2011)........................ 13

Figure 2.5: Basic Components of Performance Function.............................................. 20

Figure 2.6: Illustration of Lead gap, Lag gap, Total gap, Lead vehicle and Lag vehicle. 29

Figure 2.7: Connected Vehicles (Chong, 2016)............................................................... 30

Figure 2.8: Automated Vehicles (Chong, 2016)......................................................... 30

Figure 2.9: Summary of Safety Applications of CVs (Xiaosi et al., 2012)...................... 31

Figure 2.10: Summary of Mobility Applications (Xiaosi et al., 2012).............................. 34

Figure 2.11: CV Pilot Deployment Program Goals (USDOT, 2018)............................... 36

Figure 3.1: Image of the Study Road (Google Maps)...................................................... 44

Figure 3.2: Distribution of Gore Speeds...................................................................... 49

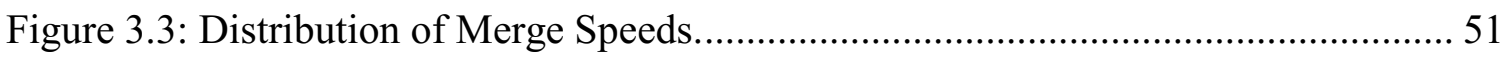

Figure 3.4: Distribution of Freeway Speeds. ........................................................... 52

Figure 3.5: Distribution of Overall Acceleration Rates................................................... 54

Figure 3.6: Traffic Volume on FRL and SCL............................................................. 56

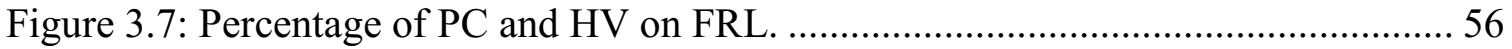

Figure 3.8: Percentage of PC and HV on SCL. ........................................................... 57

Figure 3.9: Total Collisions on Acceleration Lane during 1998 - 2002 ......................... 58

Figure 3.10: Average Annual Daily Traffic at Study Sites............................................ 59

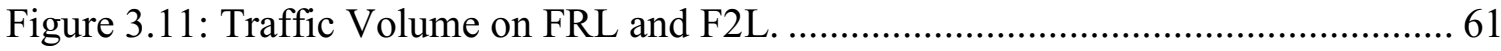




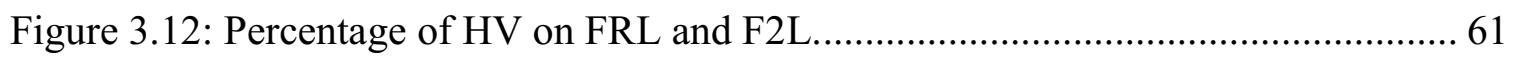

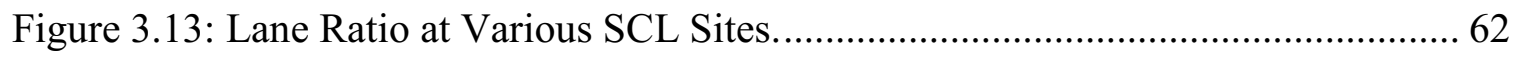

Figure 5.1: Flow Chart of the Overall Simulation Process ............................................... 73

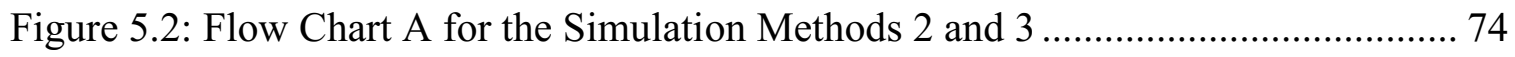

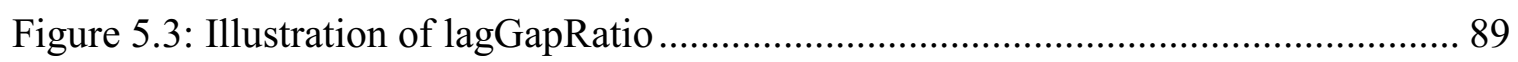

Figure 6.1: Mean PNC vs Change in Gore Speed. ……………………................. 112

Figure 6.2: Change in Mean PNC vs Change in Gore Speed....................................... 112

Figure 6.3: Mean PNC vs Change in FRL Speed. ................................................... 115

Figure 6.4: Change in Mean PNC vs Change in FRL Speed.......................................... 115

Figure 6.5: Mean PNC vs Change in F2L Speed..................................................... 117

Figure 6.6: Change in Mean PNC vs Change in F2L Speed. ......................................... 117

Figure 6.7: Mean PNC vs Change in Percentage of HV on FRL.................................. 119

Figure 6.8: Change in Mean PNC vs Change in Percentage of HV on FRL................. 119

Figure 6.9: Mean PNC vs Change in Percentage of HV on F2L.................................... 121

Figure 6.10: Change in Mean PNC vs Change in Percentage of HV on F2L................. 121

Figure 6.11: Mean PNC vs Change in FRL Traffic Volume.......................................... 123

Figure 6.12: Change in Mean PNC vs Change in FRL Traffic Volume.......................... 123

Figure 6.13: Mean PNC vs Change in Lane Ratio..................................................... 125

Figure 6.14: Change in Mean PNC vs Change in Lane Ratio. ....................................... 125

Figure 6.15: Mean PNC vs Change in SCL Length...................................................... 127

Figure 6.16: Change in Mean PNC vs Change in SCL Length..................................... 127

Figure 6.17: Mean PNC vs Change in SCL Traffic Volume.......................................... 129

Figure 6.18: Change in Mean PNC vs Change in SCL Traffic Volume........................ 129 


\section{LIST OF ACRONYMS}

$\mathrm{AADT}=$ Average annual daily traffic

AASHTO $=$ American Association of State Highway and Transportation Officials

$\mathrm{AVs}=$ Autonomous vehicles

$\mathrm{CPM}=$ Collision prediction model

$\mathrm{CVs}=$ Connected vehicles

$\mathrm{CVT}=$ Connected vehicle technology

DLC $=$ Discretionary lane change

DSRC $=$ Dedicated short-range communications

FARS $=$ Fatality analysis reporting system

$\mathrm{F} 2 \mathrm{~L}=$ Freeway second lane

FORM $=$ First order reliability method

FOSM $=$ First order second moment reliability method

$\mathrm{FRL}=$ Freeway right lane

$\mathrm{HV}=$ Heavy vehicles

ITS $=$ Intelligent transportation systems

K-S Test $=$ Kolmogorov-Smirnov test

MLC $=$ Mandatory lane change 


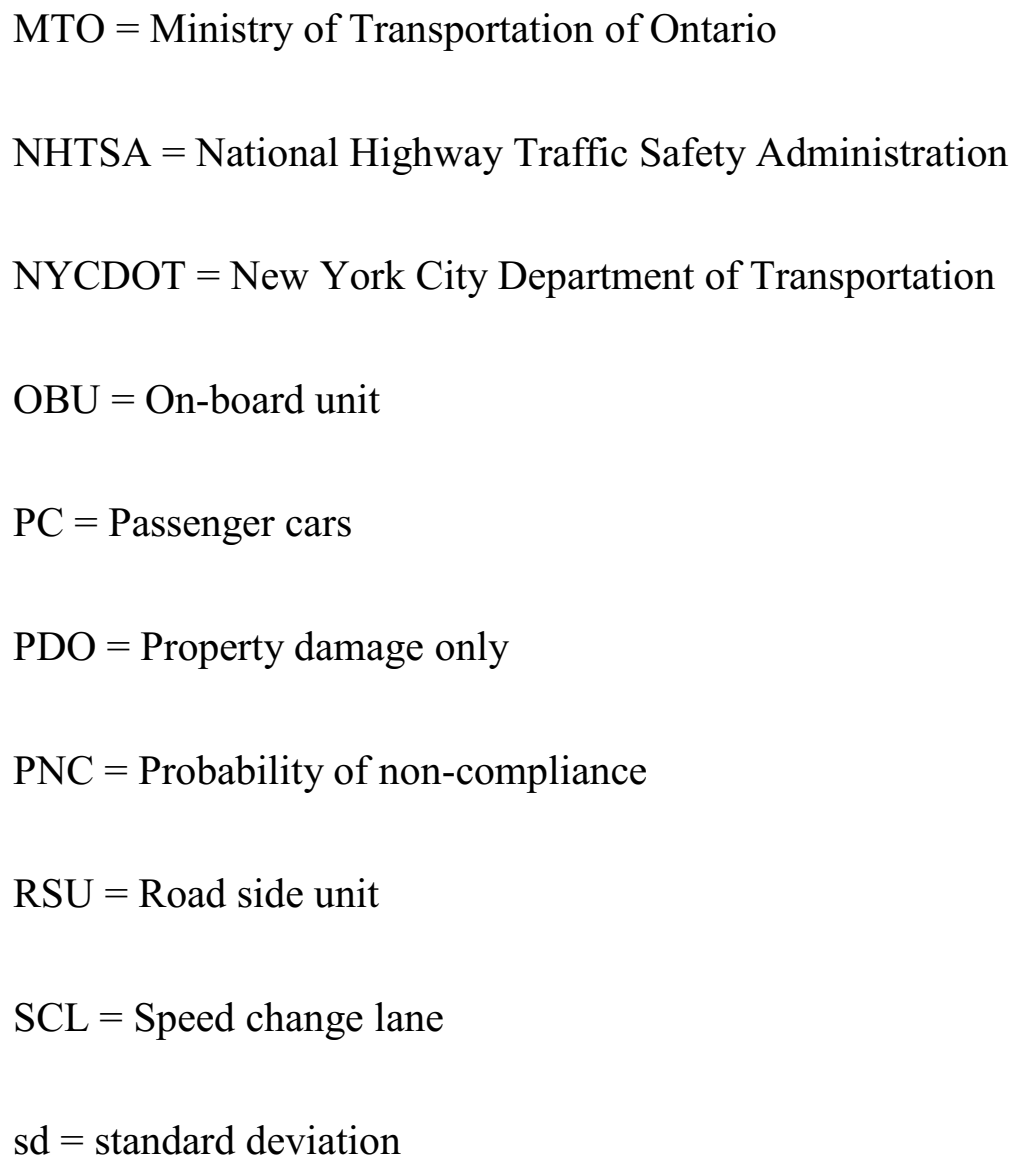




\section{Chapter 1: INTRODUCTION}

\subsection{Background}

The main function of the road network is to provide mobility to its users in a safe, convenient and efficient manner. Increasing demand for the fast and convenient means of travel resulted in severe congestion and longer waiting periods. On the other hand, high number of collisions on the road network is becoming a challenging task to the transportation engineers as they cause great loss of human lives and properties. According to Traffic Safety Facts, 2018, more than 7.27 million police-reported motor vehicle collisions occurred on roadways, about 37,461 people lost their lives in motor vehicle collisions and another 3.14 million people were injured in the United States in 2016 (National Highway Traffic Safety Administration (NHSTA), 2018). As per Canadian Motor Vehicle Traffic Collision Statistics, 2018, there were 1,717 motor vehicle fatal collisions and 115,956 personal injury collisions on roadways in 2016 (Transport Canada, 2018). Therefore, high priority is to be given to identify the causes of crashes and to find the best possible remedial methods to minimize the number of collisions. Santos et al. (2002) stated that the primary cause of vehicle crashes on the road network is driving errors, and those errors can be minimized with the proper understanding of driver's behaviour. Design of road network, high speeds, weather conditions also play a role in the occurrence of crashes along with the driver's behaviour (Santos et al., 2002).

Roadways can be classified as locals, collectors, arterials, freeways and expressways. Freeways, expressways and major arterials fall in the upper range of street classification system as per the Transport Association of Canada (TAC, 2017). According to TAC 
(2017), freeways are intended to provide high mobility and should be designed for highest practical speed in order to promote safety and efficiency. To maintain these objectives, entrance to and exit from freeways are provided at necessary intersecting roads through interchanges, and traffic flows are separated at those interchanges. Interchanges consist of a system of ramps and loops which could impact the safety of merging and diverging operations.

One of the major and very frequent problems is the merging conflicts at the freeway ramp areas which contribute a considerable portion of the vehicle crashes. Several studies stated that interchanges are highly prone to crashes in terms of both number and severity. For example, McCartt et al. (2004) stated that around 82,609 reported crashes occurred at interchanges on interstate freeways in United States (US) in the year 2001. Major portion $(83 \%)$ of interchange related crashes were observed specifically on the entrance or exit ramps of the freeways.

Exchange of traffic information has been made possible by using intelligent transportation systems (ITS). One of the recent innovations developed as a part of ITS is the connected vehicle technology (CVT). Collection of real time and detailed individual vehicular data is made possible by the advanced data collection devices in the connected vehicle (CV) environment. CVT is a technology which can allow cars to communicate with other cars, traffic signals and the other electronic devices such as mobile phones of road users, and roads can communicate with the cars in future. Vehicles equipped with the wireless communication devices, can allow communication between vehicles and their external and internal environments (Campbell, Alexiadis, \& Krechmer, 2016). 
CVT allows for the interactions of vehicle-to-vehicle (V2V) or vehicle-to-infrastructure (V2I) or vehicles to other road users (V2X) to attain greater knowledge of surroundings of the vehicle (Campbell et al., 2016). CVT is one of the promising techniques which can reduce the number of collisions and traffic congestion with the use of intelligent traffic management and control along with improved road safety and warning messages systems. CVs can possibly change the way people travel by the creation of a safe communication system between traffic signals, mobile phones of the road users and cars. Communication channels like dedicated short range communication (DSRC) facilitate the increase in the amount of information that can be exchanged. Road side units (RSU), on -road sensors like loop detectors, cameras and on-board units (OBU) would play a key role in information exchange (Guériau et al., 2016). Real-world data collected from CVs will be useful in analyzing the changes in driver behaviour and monitoring the performances of any newly installed safety devices such as pedestrian countdown signals (Jadaan, Zeater, \& Abukhalil, 2017). CVT can improve the present situation by providing the public with tools like safety warnings and information about the merging roads, dangerous curves, icy roads, and impending collisions to keep drivers and passengers away from the accidents. Research found that one of the important benefits of CVs is their capability of increasing the capacity of uninterrupted traffic flow facilities like freeways (Jadaan et al., 2017).

\subsection{Problem Definition}

There are many general factors affecting the safety performance of freeways. Traffic operation and safety performance of merging and diverging areas of a freeway can be affected by factors such as distances available for weaving, acceleration and deceleration 
(Park et al., 2001). If the length of the acceleration lane is not sufficient, drivers may not be able to complete the merging operation comfortably before reaching the end of the acceleration lane. In cases where the acceleration lane length is inadequate, drivers might have to merge with the main traffic irrespective of the available gaps for merging. These scenarios would cause main lane traffic congestion and would increase the crash probability with-in the merging area. Ample length of speed change lane (SCL) might reduce the intricacy of the problem by giving the drivers enough time to find suitable gaps and to adjust the speeds (Park et al., 2001).

Although the development and deployment of CVs is very much beneficial to the world of transportation, most of the currently available tools are limited in their capability to analyse the benefits of CVs and their applications (Campbell et al., 2016). As the penetration rate of CVs is expected to increase in the near future, demand for the development of new methodologies for traffic control and management is also being increased. A study by Hayat et al. (2016) found that the efficacy of mobility applications depends on the driver's responses to the received warning messages and understanding of the advisory messages. It was found that the perception reaction time was decreased when the advisory messages become more direct and clearer. With a lot of research going on the deployment of CVs and benefits of $\mathrm{CV}$ applications, it is also essential to identify the effective use of research data collected. CVs can collect huge amount of real-world data and that should be processed and utilised in an efficient manner to experience the real benefits of CVT.

If the acceleration lane length is short and is not enough to perform the merging process, SCL drivers can try to interact with freeway right lane (FRL) drivers to request them to 
create the gap required for the merging. FRL drivers can create the adequate gap either by adjusting their speeds or by changing their lane. But the direct interaction of SCL drivers with FRL drivers might not be possible all the time due to high speeds on FRL. However, this would be possible in a safe way by transferring the information related to SCL traffic conditions to the FRL drivers in the form of alerts through CV applications. Advisory messages can be sent to the FRL drivers to request them to adjust their speeds or to change the lanes, if it is safe to do so according to the traffic conditions on the target lane. FRL drivers can create the gaps required for SCL drivers either by adjusting the speeds or by changing the lane. In the same way, traffic conditions on the FRL can be informed to SCL drivers in order to adjust their speeds for an easy merging process. Despite having the potential to address this issue, there is no definite method to prove that this is possible through CVT and to what extent problem of merging conflicts at on-ramps can be solved by deploying the CVs. Focus of this study is to verify if the safety and efficiency of onramp merging can be improved by the complete deployment of CVs.

\subsection{Objectives}

Based on the discussions in the earlier sections, the objectives of this research were set as listed below:

- To develop a realistic simulation model representing the merging process on freeway entrance lanes considering the variations in the field data.

- To develop simulation models to assess the benefits of CVs in improving the safety at freeway on-ramps.

- To evaluate the probability of uncomfortable merging of the SCL vehicles using all 
the developed models.

- To observe the variations in the output probabilities for regular vehicles and CVs.

- To identify the relation between the probability of uncomfortable merging and the collision frequency on acceleration lanes.

- To conduct the sensitivity analysis of various input parameters

\subsection{Scope}

Scope of this study is limited to locations along the Highway 417 in and around the City of Ottawa. Due to unavailability of real-life data on how CVs behave during freeway merging process after their deployment, the scope of this study was limited to use the same data available from past researchers at the Carleton University. Even though it would take many years for the deployment of full range CVs, this study considered $100 \%$ penetration rate for CVs to evaluate the ultimate end benefit of CVs in improving the safety at freeway on-ramps.

\subsection{Thesis Organization}

An introduction to this study along with the objectives and scope have been already provided in this chapter. In Chapter 2, literature review of the previous research on freeway merging behaviour, CVT and SCL design is provided. Information about the site selection, data collection and primary data analysis are provided in Chapter 3. Regression models of the speed and acceleration parameters are presented in Chapter 4. In Chapter 5, detailed description of the methodologies and developed simulation models is presented. Results and analysis of the model outputs are discussed in Chapter 6. Conclusions and recommendations for further research are furnished in Chapter 7. 


\section{Chapter 2: LITERATURE REVIEW}

This chapter provides the literature review related to the SCL design practices according to the current design guides, freeway merging behaviour of the SCL drivers and research studies related to CVs and their benefits.

\subsection{Review of SCL Design from the Guides AASHTO (2011) and TAC (2017)}

\subsubsection{Definitions of Design Elements}

\section{(a) Interchange}

A system that interconnects roadways with one or more grade separators providing the traffic movements between roads at different levels is referred to as an interchange according to TAC (2017). Depending on the configuration, an interchange can eliminate the crossing conflicts by grade separation. It can also minimize the turning conflicts and can be effective in reducing the collisions at intersections.

As mentioned in AASHTO (2011), selecting the suitable type of interchange and its design depends on several factors such as traffic composition and traffic volume, highway classification, design speed, land use patterns, right-of-way requirements and topography of the area . Essential elements of an interchange include freeway or expressway, ramps, auxiliary lanes and cross streets. An interchange can be a single ramp connecting local road or complex layout connecting two or more highways. Figure 2.1 shows the basic interchange configurations. They can create a greater number of interchange combinations based on the scope and shape. 


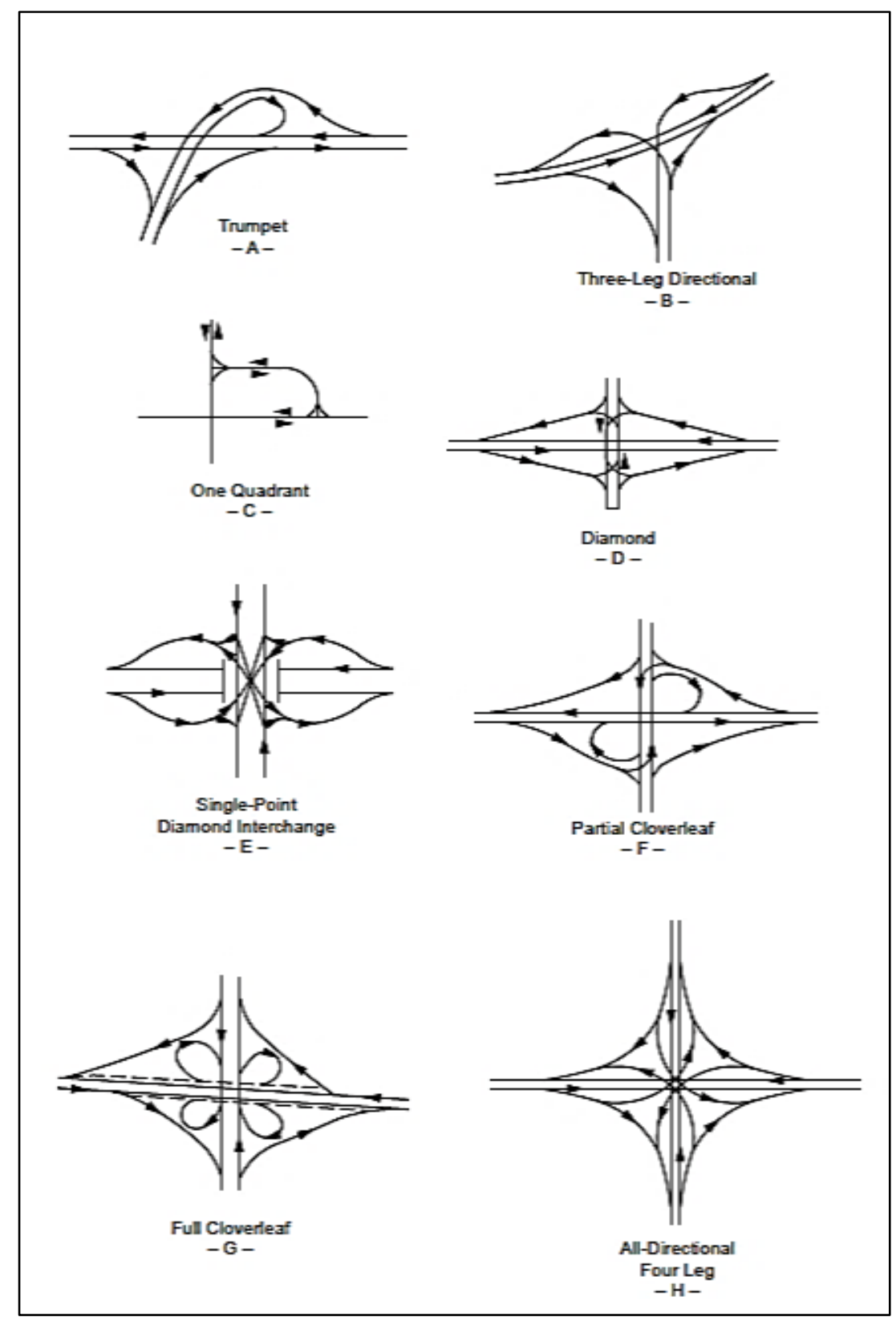

Figure 2.1: Basic Interchange Configurations (Figure 10-1, AASHTO, 2011).

\section{(b) Ramp}

According to TAC (2017), a ramp is usually one-way and connects two grade separated through roadways. An interchange ramp consists of an entrance terminal, connecting roadway and an exit terminal. Based on configuration of an interchange, the configuration 
of ramp is determined and is influenced by design considerations such as traffic volume and composition, traffic control devices installed, geometric characteristics of adjacent roads, terrain and driver expectations.

Figure 2.2 illustrates various types of ramps and their shapes, and most of the ramp configurations can broadly be classified as one of the types shown.

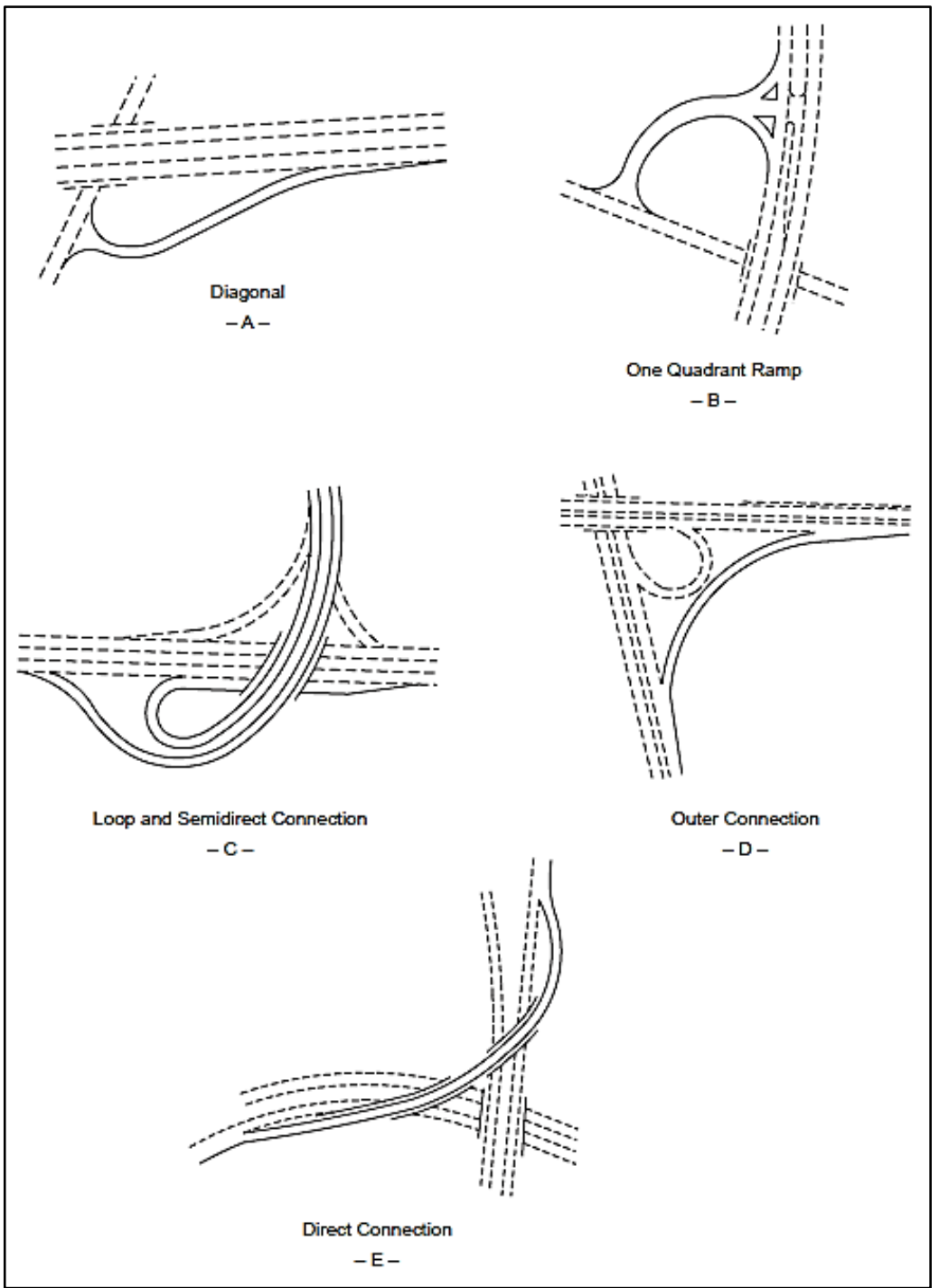

Figure 2.2: General Types of Interchange Ramps (AASHTO, 2011). 
Diagonal ramps are generally one-way, but they have a left and right turning movement on the minor intersecting roadway. Diamond interchanges usually have four diagonal ramps. A loop ramp may have both single turning movements or double turning movements at one end or at both ends. This type of ramp generally involves more indirect travel path compared with the other type of ramps. Semidirect ramp connection can provide less travel distance when compared with the direct and loop type ramp connections. In semidirect ramp connection, drivers exit and travel away from the travel path and gradually pass other interchange ramps before entering into the desired road.

It is not often feasible to provide ramp design speeds closer to the design speed of through roadways, but design speed of the ramps depends on the design speed of interesting roads. Due to site limitations, lower design speeds compared to freeway design speed are generally used for ramps. Table 2.1 shows the TAC (2017) recommendations for design speed of ramps based on the through roadway design speed.

Table 2.1: Ramp Design Speed (TAC, 2017).

\begin{tabular}{|c|c|}
\hline Through Roadway Design Speed $(\mathrm{km} / \mathrm{h})$ & Ramp Design Speed $(\mathrm{km} / \mathrm{h})$ \\
\hline 60 & $50-40$ \\
\hline 70 & $60-40$ \\
\hline 80 & $70-40$ \\
\hline 90 & $80-50$ \\
\hline 100 & $90-50$ \\
\hline 110 & $100-60$ \\
\hline 120 & $110-60$ \\
\hline 130 & $110-70$ \\
\hline
\end{tabular}


Depending on the site controls, configuration of interchange, traffic composition and environmental characteristics, a ramp design speed value between lower and upper limits will be applicable.

\section{(c) Gore}

The area between a through roadway and an entrance ramp is referred to as gore (TAC, 2017). The point separating the roadways having some dimensional width upstream from the gore is called as physical nose whereas a painted nose is a point with no dimensional width present at the separation of roadways. The triangular area between the gore nose and the painted nose is called as neutral area. Figure 2.3 shows the layout of a typical entrance gore area components, which was reproduced from the Figure 10-61 of AASHTO (2011).

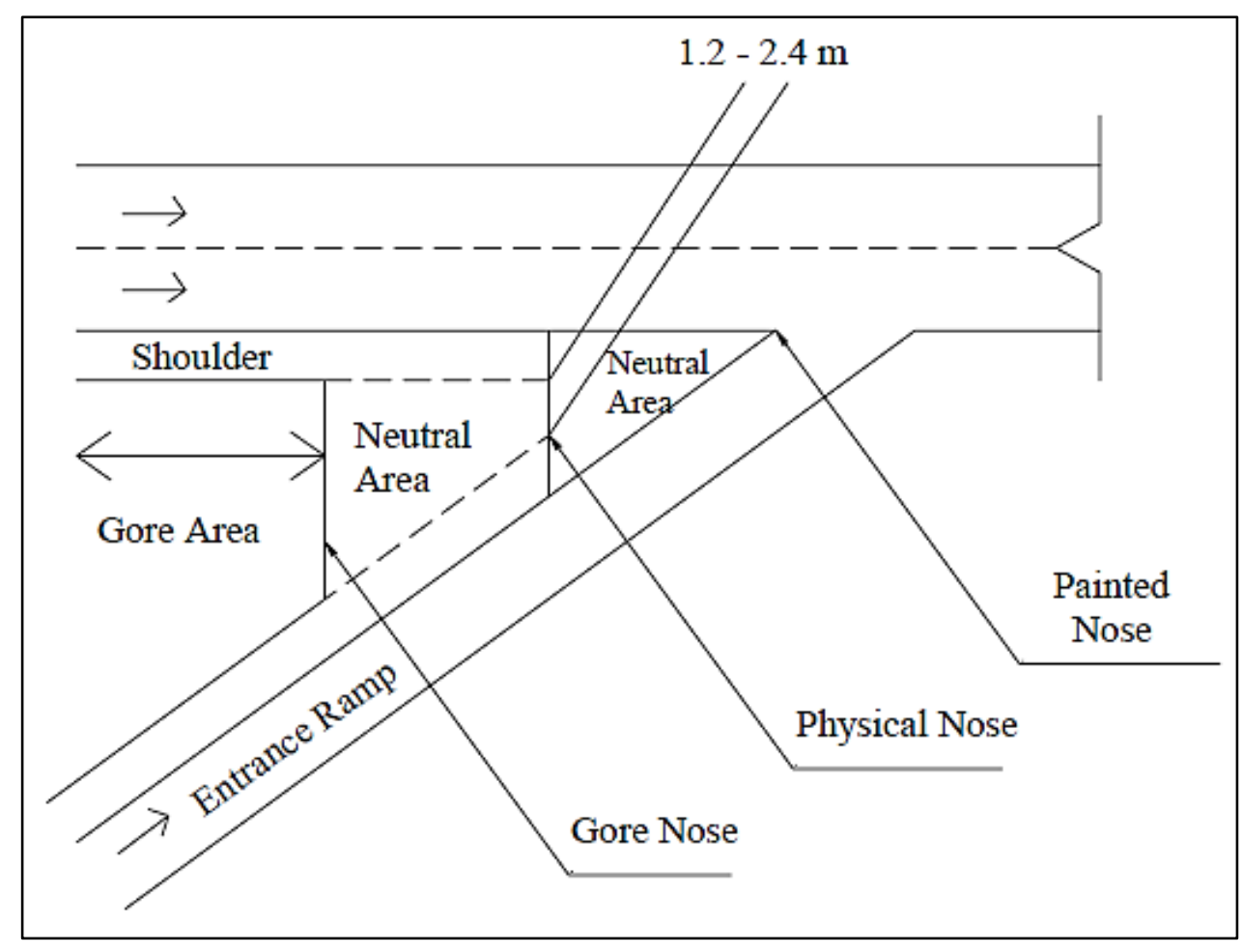

Figure 2.3: Typical Entrance Gore Area (AASHTO, 2011). 
Geometric layout of these components is an important part and should be clearly visible to drivers. In a series of interchanges along a freeway, the gores should provide similar appearance to drivers and should be uniform (TAC, 2017).

\section{(d) Speed Change Lane}

At an interchange, if drivers are leaving the highway, it is necessary to slow down as they exit from a ramp. Similarly, if the drivers are entering a freeway, they are required to increase the speed until they reach the desired highway speed. Because the speed difference between ramp and highway is considerably large in most cases, it is necessary to provide some auxiliary lanes to accelerate or decelerate. These auxiliary lanes can also reduce conflicts with through traffic and crash frequency. Such an auxiliary lane is referred to as a speed change lane (SCL) (AASHTO, 2011). A SCL, either an acceleration lane or a deceleration lane, should offer enough length to the drivers to make the required change in speed between highway and ramp. According to TAC (2017), length of a SCL depends on three factors: highway speed, speed of the ramp and manner of accelerating or decelerating.

Two common forms of SCL are there: the taper type and the parallel type (AASHTO, 2011). Taper type offers a direct exit or entry at a flat angle, whereas the parallel type SCL has an additional lane for the speed change. Some agencies prefer parallel type for entering and taper type for exiting. Figure 2.4 shows the illustration of typical single lane entrance ramps of taper type and parallel type. 


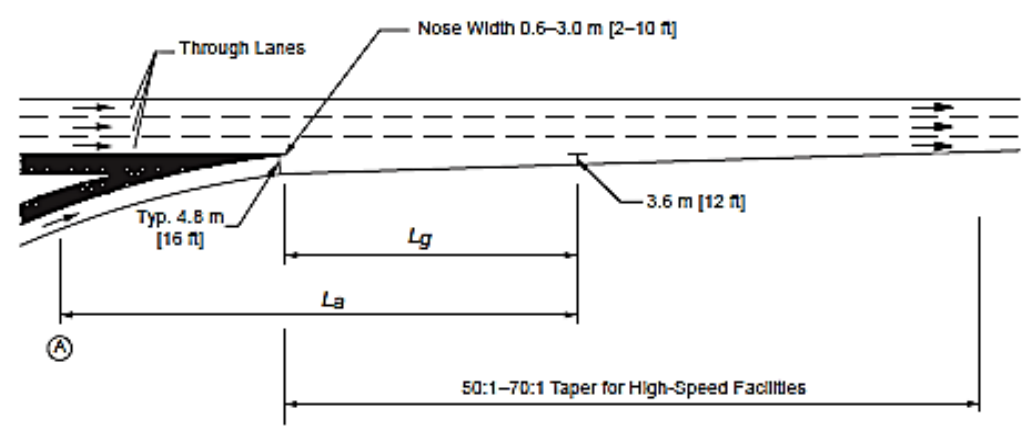

Tapered Design

$-\mathrm{A}-$

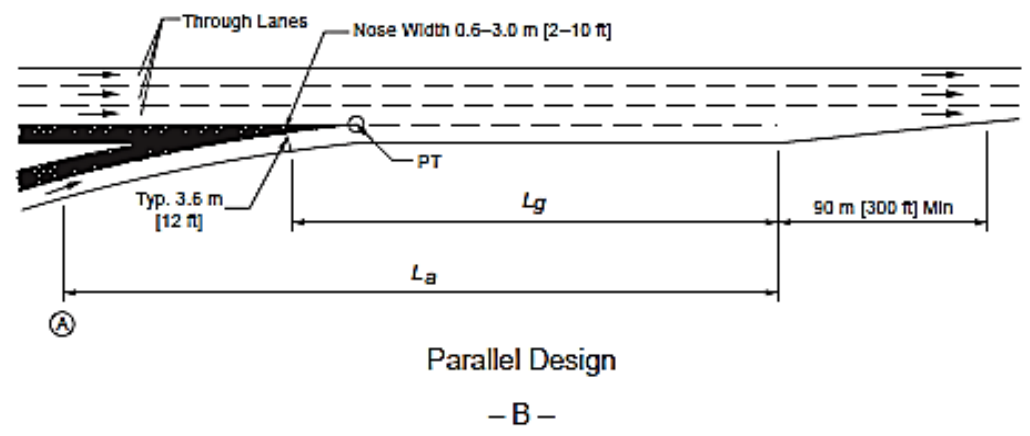

Figure 2.4: Taper and Parallel Type Entrance Ramps (AASHTO, 2011).

As mentioned in TAC (2017), the process of merging into freeway traffic is similar to making a lane change to left. The parallel design allows longer lengths of acceleration lanes thereby providing more flexibility and time for drivers to find a suitable gap to merge into the through traffic. This safety and operational benefit of the parallel type SCL is more relevant in the case of shorter headways and infrequent gaps between heavy traffic volumes. On the other hand, taper type design has lesser property requirements and provides a direct path for merging. However, it offers shorter acceleration lengths compared to the parallel type design, which is a problem in high traffic volume conditions (TAC, 2017). 


\subsubsection{Design Guidelines for Acceleration Lane Length}

According to the design guide TAC (2017), the length of an acceleration lane depends on several factors such as manner of acceleration, through traffic details and speed of ramp controlling curve. Also, the length of an acceleration lane depends on the distance required to increase speed to the desired highway speed and the distance required to complete safe merging action. For the high traffic volumes of through lanes, longer entrance terminals are desirable to perform a safe and convenient merging operation. Buses and trucks require longer lengths for accelerating compared to those required for passenger cars. Table 2.2 shows the design recommendations by TAC (2017) for acceleration lane lengths.

Table 2.2: Design Length for Acceleration Lane (TAC, 2017).

\begin{tabular}{|c|c|c|c|c|c|c|c|c|c|c|}
\hline \multicolumn{2}{|c|}{ Speed of Roadway } & \multirow{3}{*}{$\begin{array}{l}\text { Length } \\
\text { of } \\
\text { Taper } \\
\text { (m) }\end{array}$} & \multirow{2}{*}{\multicolumn{8}{|c|}{$\begin{array}{l}\text { Length of Acceleration Lane Excluding Taper (m) } \\
\text { Design Speed of Turning Roadway Curve }(\mathrm{km} / \mathrm{h})\end{array}$}} \\
\hline \multirow{2}{*}{$\begin{array}{l}\text { Design } \\
(\mathrm{km} / \mathrm{h})\end{array}$} & \multirow{2}{*}{$\begin{array}{l}\text { Assumed } \\
\text { Operating } \\
(\mathrm{km} / \mathrm{h})\end{array}$} & & & & & & & & & \\
\hline & & & $\begin{array}{c}\text { Stop } \\
\text { Condition } \\
\end{array}$ & 20 & 30 & 40 & 50 & 60 & 70 & 80 \\
\hline 60 & $55-60$ & 55 & $85-115$ & $70-100$ & $60-80$ & $45-60$ & $20-35$ & - & - & - \\
\hline 70 & $63-70$ & 65 & $120-60$ & $115-150$ & $100-135$ & $80-115$ & $50-85$ & $15-40$ & - & - \\
\hline 80 & $70-80$ & 70 & $160-225$ & $150-215$ & $130-200$ & $115-185$ & $85-160$ & $40-100$ & - & - \\
\hline 90 & $77-90$ & 80 & $215-325$ & $200-310$ & $180-300$ & $160-285$ & $140-250$ & $50-200$ & $40-145$ & \\
\hline 100 & $85-100$ & 85 & $275-450$ & $250-440$ & $210-420$ & $225-405$ & $200-375$ & $140-325$ & $100-285$ & $40-230$ \\
\hline 110 & $91-100$ & 90 & $330-650$ & $320-645$ & $305-630$ & $290-600$ & $260-575$ & $210-525$ & $150-475$ & $100-410$ \\
\hline 120 & $98-120$ & 95 & $410-730$ & $400-725$ & $375-710$ & $370-690$ & $340-660$ & $285-590$ & $250-515$ & $135-430$ \\
\hline 130 & $105-130$ & 100 & $550-885$ & $540-880$ & $510-870$ & $500-850$ & $470-820$ & $400-745$ & $340-655$ & $300-550$ \\
\hline
\end{tabular}


Minimum lengths of acceleration lanes recommended by AASHTO (2011), which are based on the design speed and speed of ramp controlling curve, are shown in Table 2.3. For parallel type ramp, length of acceleration lane is measured from the end of ramp controlling curve to the beginning of the taper. For the direct ramp type, the length is measured from the end of the ramp controlling curve to the point where the width of auxiliary lane is $3.6 \mathrm{~m}$ (AASHTO, 2011). An acceleration lane length of minimum $360 \mathrm{~m}$ in addition to the taper is required wherever it is estimated that the freeway and ramp will frequently carry traffic volumes close to the design capacity of the merging area. A taper length of approximately $90 \mathrm{~m}$ is appropriate for design speeds up to $110 \mathrm{~km} / \mathrm{h}$ to guide the vehicles onto the freeway through lane.

Table 2.3: Minimum Acceleration Lengths for Entrance Terminals (AASHTO, 2011).

\begin{tabular}{|c|c|c|c|c|c|c|c|c|c|c|}
\hline \multicolumn{10}{|c|}{ Acceleration Length (m) for Entrance Curve Design Speed (km/h) } \\
\hline \multicolumn{2}{|c|}{ Highway } & $\begin{array}{c}\text { Stop } \\
\text { Condition }\end{array}$ & 20 & 30 & 40 & 50 & 60 & 70 & 80 \\
\hline $\begin{array}{c}\text { Design } \\
\text { Speed } \\
(\mathrm{km} / \mathrm{h})\end{array}$ & $\begin{array}{c}\text { Speed } \\
\text { Reached } \\
(\mathrm{km} / \mathrm{h})\end{array}$ & 0 & 20 & 28 & 35 & 42 & 51 & 63 & 70 \\
\hline 50 & 37 & 60 & 50 & 30 & - & - & - & - & - \\
\hline 60 & 45 & 95 & 80 & 65 & 45 & - & - & - & - \\
\hline 70 & 53 & 150 & 130 & 110 & 90 & 65 & - & - & - \\
\hline 80 & 60 & 200 & 180 & 165 & 145 & 115 & 65 & - & - \\
\hline 90 & 67 & 260 & 245 & 225 & 205 & 175 & 125 & 35 & - \\
\hline 100 & 74 & 345 & 325 & 305 & 285 & 255 & 205 & 110 & 40 \\
\hline 110 & 81 & 430 & 410 & 390 & 370 & 340 & 290 & 200 & 125 \\
\hline 120 & 88 & 545 & 530 & 515 & 490 & 460 & 410 & 325 & 245 \\
\hline
\end{tabular}

Note: Uniform 50:1 to 70:1 tapers are recommended where the length of acceleration lanes exceed $400 \mathrm{~m}$. 
As shown in Table 2.3, speed reached by entering vehicles at the end of recommended length is 13 to $32 \mathrm{~km} / \mathrm{h}$ lower than the design speeds of highway, which varies between 50 and $120 \mathrm{~km} / \mathrm{h}$. The guide also recommends that the geometry of taper type entrance ramps should allow drivers to reach a speed within $10 \mathrm{~km} / \mathrm{h}$ range from the design speed of the freeway by the time they reach the location where edges of the freeway through lane and ramp are $3.6 \mathrm{~m}$ apart. This inconsistency in the recommendations of the guide may lead to differences between the operating speed of freeways and merging speeds of SCL vehicles which might also impact crash frequency at the merging areas.

\subsection{Previous Research on Acceleration Length Design}

AASHTO (2011) assumes operating speed of freeway which is 13 to $32 \mathrm{~km} / \mathrm{h}$ lower than the design speed of the freeway and TAC (2017) assumes operating speed which is 5 to 25 $\mathrm{km} / \mathrm{h}$ lower than the design speed. However, Seneviratne and Islam (1992) found that the operating freeway speed sometimes exceeds the design speed of freeway. Similarly, Ahammed et al. (2008) noticed that some merge speeds of passenger cars are higher than the posted speed limit of the freeway. AASHTO (1965) stated that the length of the acceleration lane was based on the speed at the end of ramp controlling curve, the manner of drivers' acceleration and the merge speed of drivers. Although, AASHTO (2004) AASHTO (2011) did not explicitly explain how the recommended SCL length values were obtained, but these values closely matches with the values in AASHTO (1965) guide. In general, the acceleration lanes were used not only for increasing the speed but also for searching a suitable gap for merging into freeway traffic. Thus, the assumptions used in the current design guides would not lead to the accurate estimation of acceleration lane 
lengths and do not consider the gap searching behaviour of SCL drivers in design SCL length recommendations.

As per Fitzpatrick and Zimmerman (2007), AASHTO (1965) design guide used Equation 2.2 to calculate the acceleration lane length.

$$
L=\frac{\left(0.278 V_{1}\right)^{2}-\left(0.278 V_{2}\right)^{2}}{2 a}
$$

Where

$L=$ acceleration lane length $(\mathrm{m})$

$V_{l}=$ operating speed of the highway $(\mathrm{km} / \mathrm{h})$

$V_{2}=$ operating speed on entrance curve of the ramp $(\mathrm{km} / \mathrm{h})$

$a=$ acceleration rate $\left(\mathrm{m} / \mathrm{s}^{2}\right)$

Fitzpatrick and Zimmerman (2007) also mentioned that the current North American design guides consider the acceleration rate extracted from the studies conducted between 1930 and 1940 for the design purposes. Fitzpatrick and Zimmerman (2007) suggested acceleration lengths that are based on the realistic acceleration distances and speed assumptions. Note that the suggested lengths of acceleration lanes were longer than those recommended by AASHTO (2011) for corresponding design and operating speed values.

Hassan et al. (2012) presented a probabilistic approach for determining the length of acceleration lane lengths. A reliability-based model was developed to determine the distribution of drivers' acceleration distances on SCLs. This distribution could be used to 
calculate the appropriate SCL length for a new design or could be used for evaluating an existing design. Drivers gap searching and acceptance behaviour was not explicitly considered in the analysis of acceleration distances using analytical method and simulation technique. Analytical method suggested Equation 2.2 for estimating the acceleration distance on SCLs. It was also recommended that the design SCL length can be considered as $85^{\text {th }}$ or $95^{\text {th }}$ percentile value of acceleration distance calculated from Equation 2.2.

$$
E(L)=\frac{\left(0.278 \mu_{V M}\right)^{2}-\left(0.278 \mu_{V G}\right)^{2}}{2 \mu_{a}}
$$

Where

$E(L)=$ estimated acceleration distance $(\mathrm{m})$

$\mu_{V M}=$ mean merge speed $(\mathrm{km} / \mathrm{h})$

$\mu_{V G}=$ mean gore speed $(\mathrm{km} / \mathrm{h})$

$\mu_{a}=$ mean acceleration rate $\left(\mathrm{m} / \mathrm{s}^{2}\right)$

Yang et al. (2016) developed a method to determine the acceleration lane lengths at metered entrance ramps. Vehicles location and time data were collected at seven metered on-ramps and regression models were developed to estimate the acceleration lane lengths at a given merge speed. The $85^{\text {th }}$ percentile value of the estimated lengths was recommended as a minimum acceleration SCL length in order to accommodate the drivers in reaching a safe merge speed. It was found that the recommended SCL lengths for PC were 10 to $35 \%$ shorter than those recommended by AASHTO (2011) guide. It was also 
found that the minimum acceleration lane lengths required for heavy trucks were approximately $60 \%$ greater than those recommended by AASHTO (2011) guide.

\subsection{Reliability Concept in Geometric Design}

As stated by Ismail and Sayed (2009), the North American design guides adopt a deterministic approach to address the uncertainty in input design parameters by considering single values. However, the highway geometric design is mainly based on human factors, road conditions, vehicle properties and performance of a roadway system, which are not constant. Thus, consideration of a probabilistic approach for geometric design is essential for adequate safety level of a highway network. This approach involves the probabilistic assessment of a system performance in all possible conditions. Unlike the deterministic approach where each variable has only single value, the probabilistic approach considers the statistical distribution of the variables used in the design. As said by Dhahir (2018), the probabilistic approach could provide quantitative evaluation of safety in terms of probability of failure or reliability index.

Reliability analysis has gained a great attention in various engineering fields. In transportation engineering the application of reliability theory was comparatively limited. However, recent research studies such as Easa (2000), Richl and Sayed (2006), Khoury \& Hobeika (2007), Sarhan (2008), Dhahir (2018) have shown the potential benefits of reliability analysis in geometric design of the road elements. According to Singh et al. (2007), a system's reliability can be practically measured in terms of probability and it is defined as the probability of nonfailure. The failure of a system occurs when the demand (D) exceeds the supply (S), by which the system fails to perform its intended task. 
As stated by Ismail and Sayed (2009), in reliability analysis a performance function is constructed in such a way that it results positive output when the system is safe and negative output when system performance is unfavorable. The performance function commonly indicates the difference between the supply (S) and demand (D). In highway geometric design, supply means the set of input design parameters accounting for comfortable and safe driving conditions. Demand denotes the driver and vehicle requirements that need to be fulfilled by the design. Demand and supply are uncertain in nature and the hatched portion $\left[1-F_{D}(x)\right]$ in Figure 2.5 represents the probability that the demand is exceeding a given value of $x$, where $f_{s}(x)$ is the density function of supply (Sarhan, 2008).

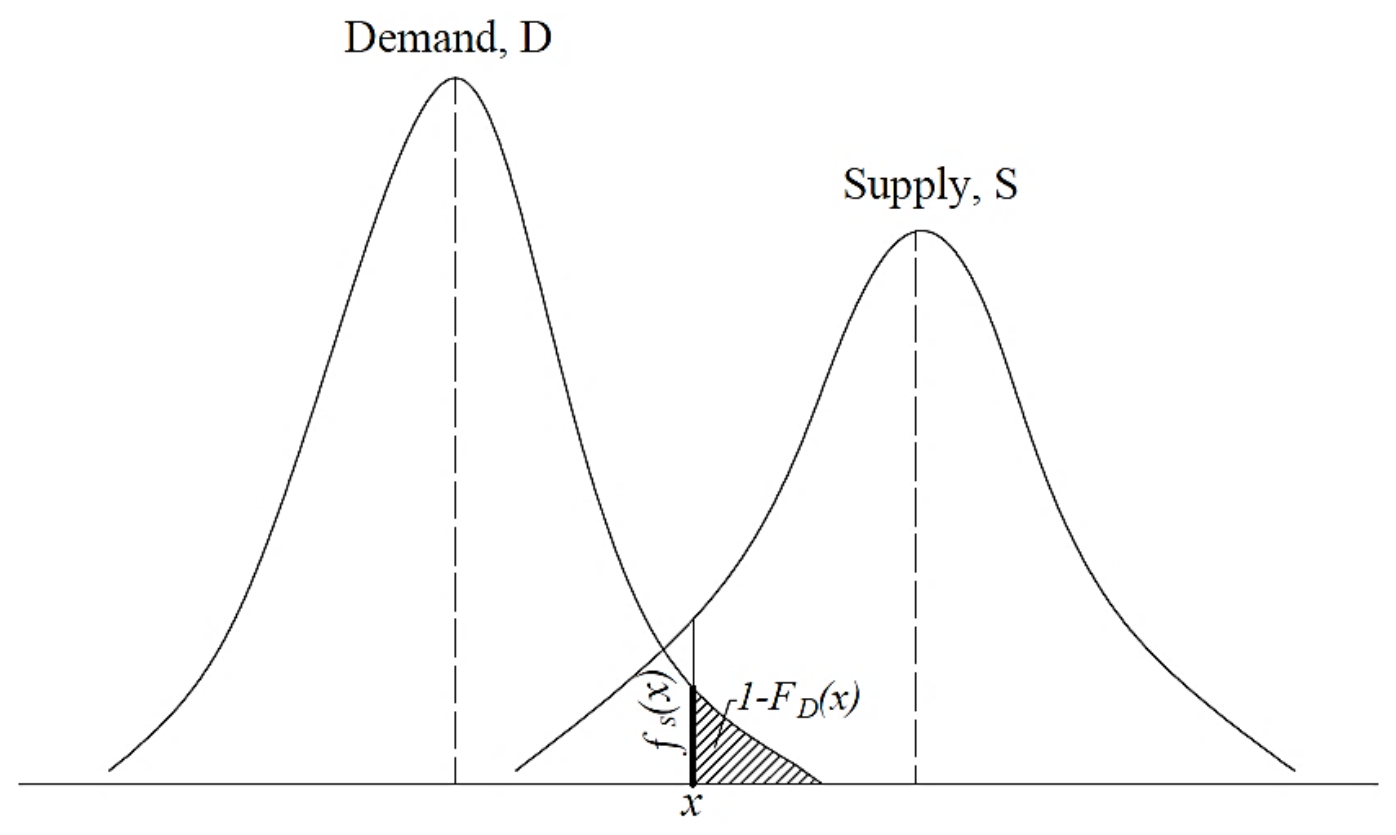

Figure 2.5: Basic Components of Performance Function. 
According to Sarhan (2008), probability of non-compliance (PNC) at a given value of $x$ is the product of $f_{s}(x)$ and $\left[1-F_{D}(x)\right]$. By integrating this product from zero to infinity, the overall PNC can be estimated as following:

$$
P N C=\int_{0}^{\infty} f_{S}(x)\left[1-F_{D}(x)\right] d x
$$

The term PNC has been suggested in the literature related to highway geometric design for being more meaningful indicator of safety because of the absence of a physical failure in real-world. PNC is a distinct indicator of risk involving only the uncertainty in the design components (Ismail \& Sayed, 2009).

The probability from Equation 2.3 can be calculated by several methods such as first order reliability method (FORM), first order second moment reliability method (FOSM) or by exact integration using numerical methods. The exact method considers the shape of distribution of design variables in the performance function and this method uses numerical methods such as Monte Carlo Simulation technique. In the FORM method, only the mean and variance of the distribution of parameters are considered instead of shape of the distribution function. This method is relatively simple in calculations as it uses the truncated Taylor series expansion. In FOSM method, each variable is assumed to follow a normal distribution. FORM attempts to transform the distribution of all the design variables to a standard normal distribution (Singh et. al., 2007).

After having discussed the design guidelines for SCL design, existing studies on acceleration lane length design and reliability analysis in highway geometric design, the next section presents some of the existing research studies on the freeway merging 
behaviour of SCL drivers. This includes the studies on gap acceptance and acceleration behaviour of SCL drivers at freeway acceleration lanes, lane changing behaviour of freeway main lane drivers and variations in SCL drivers speed parameters, in vehicles without any connectivity.

\subsection{Previous Research on Freeway Merging Behaviour}

AASHTO (2011) stated that "a speed-change lane should have sufficient length to enable a driver to make the appropriate change in speed between the highway and the turning roadway. Moreover, in the case of an acceleration lane, there should be additional length to permit adjustments in speeds of both through and entering vehicles so that the entering driver can position the vehicle opposite a gap in the through-traffic stream and then maneuver into the stream before the acceleration lane ends." Even though, the design guide mentioned the gap in the through lane of the freeway, the same not been considered as a quantitative measure in the process of designing the SCL length. In real world merging scenario, the merging process is based on the driver's acceleration and gap acceptance behaviour. Polus et al. (1985) found that most of the SCL drivers on the acceleration lanes use the SCL length not only for acceleration purpose, but for searching the suitable gap and making decisions on accepting the available gaps. Freeway merging process is mainly associated with the gore speed, acceleration rate, merge speed, available gaps in the FRL, speed of FRL vehicles and FRL traffic volume. Ben-Akiva et al. (1985) introduced a probabilistic approach in highway geometric design in which distributions of design values have been considered instead of a single value for each design parameter. The designers can select an appropriate design from the set of output values based on the safety, economy and other factors. 
Gipps (1986) developed a lane changing decision model, which could be used in the traffic simulation. Various urban driving behaviours where the lane changing action is affected by the circumstances like the presence of traffic signal, heavy vehicle or any other sort of obstruction were covered. It was observed that factors like desirability, safety influence, and necessity might influence the decisions of lane change. Variations in the freeway driver's behaviour were not considered in this model.

A probabilistic lane changing framework was developed by Yang Qi and Haris (1996), which was applicable to freeways. In this framework, both mandatory lane change (MLC) and discretionary lane change (DLC) were analysed. Two conditions were inspected in this model for DLC scenario: whether existing speed of the adjacent lanes is high enough to increase the speed or the speed of the lead vehicle is less than the required speed. Also, a model for gap acceptance was developed and it was found that the critical gap size was smaller for MLC scenario when compared with DLC scenario.

Ahmed (1999) developed a lane changing model for different lane changing scenarios for both MLC and DLC. The developed models involved four stages namely decision of lane changing, selecting a target lane, choosing a target gap, and performing the lane change action. Maximum likelihood estimation method was used to estimate the parameters of MLC and DLC. It was observed that the driver's behaviour is more aggressive in MLC scenario.

Kita (1999) framed a merging model considering the two-way interactions between through and merging vehicles. Conditions like driver merging into the mainstream or staying in the acceleration lane were observed. Also, two options were considered for 
through vehicle such as whether it is co-operative for merging or not. Using the maximum likelihood method, the probability of merging and the probability of changing the lane were estimated.

Hunter et al. (2001) investigated the speed-distance relationship with the collected data from six ramps in Texas, which have the SCL lengths varying from $55 \mathrm{~m}$ to $241 \mathrm{~m}$. Distance traveled by vehicle and time taken to travel the distance were used to calculate vehicle speed. It was noticed that the geometry of the freeway ramp has a considerable impact on the acceleration and deceleration rates of the merging vehicle. It was also observed that 85 percent of the drivers were driving at $70 \%$ to $80 \%$ of the freeway design speed.

Hwang and Park (2005) modeled the factors affecting the gap acceptance behaviour by utility function. Real field data was used to estimate the variables and it was observed that the lag gap is a more significant parameter than the lead gap for merging. Also, it was noticed that the drivers accept smaller gap sizes when there was a traffic congestion.

Li et al. (2007) examined the drivers merging behaviour on the acceleration lanes of the freeway using the field data. It was noticed that the majority of the vehicles merge at the midway of the acceleration lane. The input parameters considered for deriving the probability equations were critical gap of merge vehicle, speed and acceleration rate of the vehicle, traffic volume, and length of the acceleration lane. However, driver's behaviour on the acceleration lanes was not considered. It was also observed that the traffic volume also has a noteworthy influence on the merging distance. 
Using the speed data collected from fifteen interchanges, Ahammed et al. (2008) observed the variations in driver's merge speed, gore speed, merging distance and the acceleration rate. It was found that $85^{\text {th }}$ percentile of the merge speed was ranging between $90.6 \mathrm{~km} / \mathrm{h}$ and $115.1 \mathrm{~km} / \mathrm{h}$ whereas the speed limit on the freeway was $100 \mathrm{~km} / \mathrm{h}$. It was also noticed that $85^{\text {th }}$ percentile of the acceleration rate ranged between $0.235 \mathrm{~m} / \mathrm{s}^{2}$ and $1.110 \mathrm{~m} / \mathrm{s}^{2}$.

Brewer et al. (2011) analyzed the merging behaviour of the drivers on the acceleration lanes with the data collected from nine entrance ramps. It was noticed that a minimum 50\% of the acceleration lane length was used for performing the merging action under low traffic congestion conditions.

Fatema and Hassan (2013) used microsimulation method to capture vehicles movement from freeway entrance SCL to through lanes of the freeway. Monte Carlo simulation method was used to examine the variations in SCL driver's acceleration and gap acceptance approaches. In this research, the probability of non-compliance (PNC) was estimated, which is the probability of a forced merge for each vehicle on the SCL. It was also noticed that the traffic volume on the freeway right lane has a considerable impact on the process of merging. From the sensitivity analysis, it was found that the mean PNC value was more sensitive with the acceleration rates and the length of SCL as compared with the other parameters.

Fatema, Ismail and Hassan (2014) analysed characteristics of merging behaviour based on the actual gap selection approaches of SCL drivers. A probabilistic model for examining the merging process, in which the gap search process is either continuous or the merging occurs at random points along the SCL, was introduced in this study. Various probability 
distributions were examined to understand the SCL driver's decisions on acceptable gap for merging. Collision frequency was related to different traffic volumes and driver's characteristics during the merging process and then it was related to the PNC for on-ramp merging.

This section summarizes the importance of driver's gap acceptance and acceleration behaviour in the design of SCL. Also, this section emphasizes the importance of parameters like merge speed, traffic volume, acceleration rate and the SCL length for the safer merging process.

\subsection{Definitions of Terms}

Various types of gaps and types of freeway vehicles used in this study were defined in this section.

\section{Relative Distance}

The ratio of SCL vehicle merging location to the SCL length is referred to as relative distance in this study.

Accepted Gap

In the present study, accepted gap is the gap which was accepted by the SCL driver to complete the merging action.

Acceptable Gap

Acceptable gap is the gap which can offer the comfortable merging to each SCL driver to merge into the freeway traffic. 


\section{Lane Changing}

The vehicle movement from one lane to another lane of the freeway is referred to as a lane change action. Based on the implementation of the lane change action, it can be categorized into two types: mandatory lane change (MLC) and discretionary lane change (DLC). If the driver is necessitated to perform a lane change in order to be in a constant travel path, it is referred to as MLC whereas DLC is optional for the driver. If the driver is willing to change the lane to create necessary gap for SCL drivers or changed the lane due to any unfavorable situations ahead, it is referred to as DLC (Zhao et al., 2014).

\section{Headway and Gap}

The time interval between two successive vehicles which are moving in the same direction, is referred to as headway. Headway is measured from the front of the lead vehicle to the front of the next arriving vehicle, whereas the gap is measured from the rear of the lead vehicle to the front of the next following vehicle.

\section{Critical Headway}

The headway of the freeway lag vehicle at which it has to start decelerating in order to create sufficient gap for the SCL vehicle.

\section{Subject Vehicle}

A vehicle on the SCL, which is trying to merge into the freeway through traffic and is searching for a suitable gap, is referred to as subject vehicle. 


\section{Freeway Lag Vehicle}

A freeway vehicle which is behind the subject vehicle, when viewing from the subject vehicle is referred to as freeway lag vehicle.

Lag Gap

The gap measured between the rear end of the subject vehicle and the front end of the lag vehicle is referred to as the lag gap.

Freeway Lead Vehicle

A freeway vehicle which is right in front of the subject vehicle when viewing from the subject vehicle is termed as freeway lead vehicle.

\section{Lead Gap}

The gap measured between the rear end of the lead vehicle and front end of the subject vehicle is termed as lead gap.

\section{Total Gap}

The gap measured between the rear end of the lead vehicle and front end of the lag vehicle is termed as total gap in this study.

An illustration of lead gap, lag gap, total gap, lead vehicle and lag vehicle is provided in Figure 2.6. 


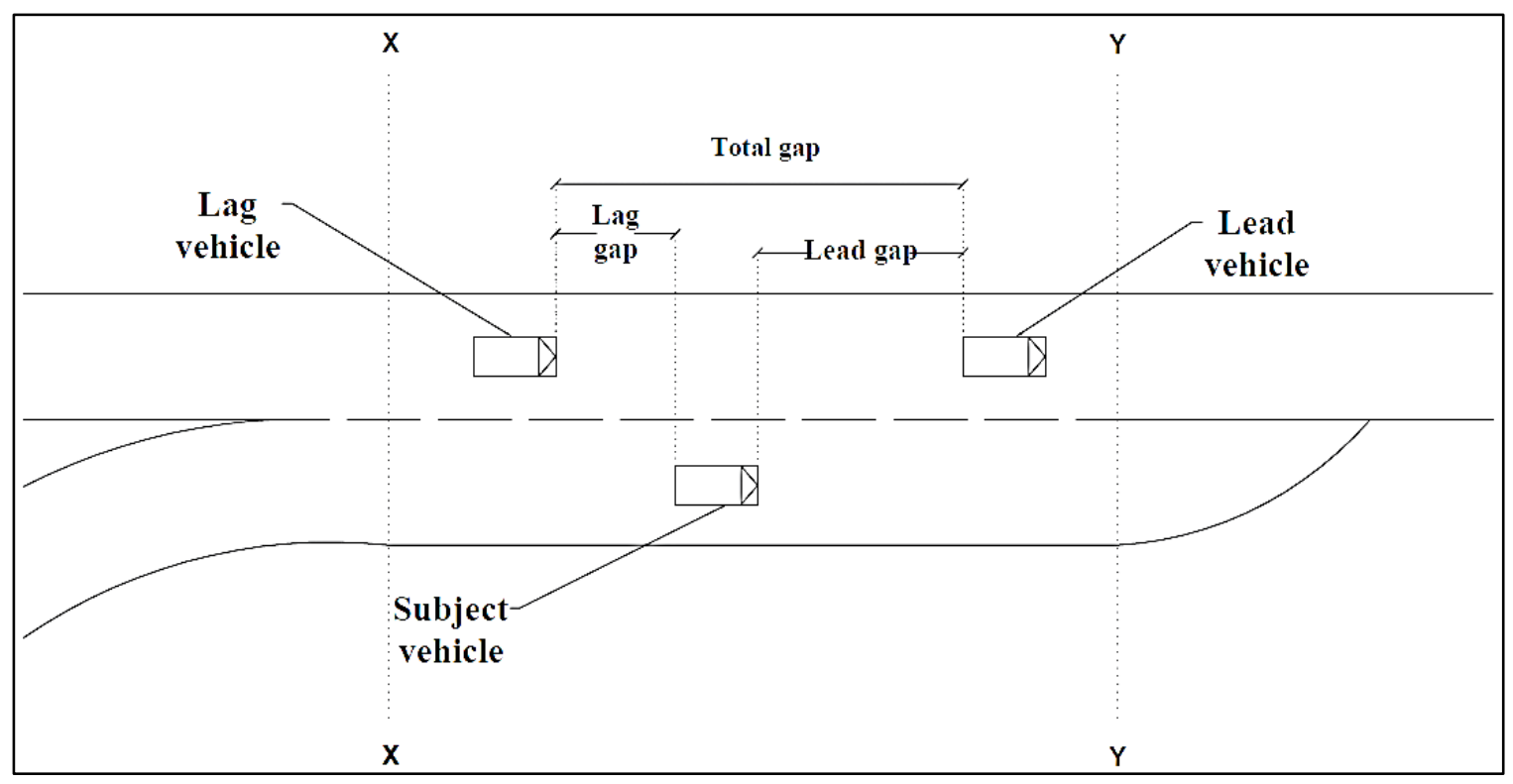

Figure 2.6: Illustration of Lead gap, Lag gap, Total gap, Lead vehicle and Lag vehicle.

\subsection{Connected Vehicle Technology}

As mentioned in Section 1.1, CVs use wireless communication technology to connect with the surrounding vehicles (V2V), infrastructure (V2I) and other mobile devices (V2X) as shown in Figure 2.7. Automated Vehicles (AVs) use sensing technologies onboard such as cameras, radars instead of vehicle communications to provide the information about the surroundings as shown in Figure 2.8 (Campbell et al., 2016). As stated by Chong (2016), CVs and AVs use some of the same technologies which can be combined in the same vehicle, however these terms are not synonymous. CVT allows vehicles to share the information about their speed, location, direction, brake status and other details to the other CVs through V2V connectivity. Exchange of information about traffic signals, rail grade crossings, and roadway signs can be through V2I connectivity. This information can be shared to the drivers and alert then about any dangerous situations ahead. In the case of 
AVs, there are varying levels of automation based on the performance of the automated driving system in the vehicle. While CVT is not necessary for the operation of AVs at every moment, it will help AVs in achieving some of the intended tasks.

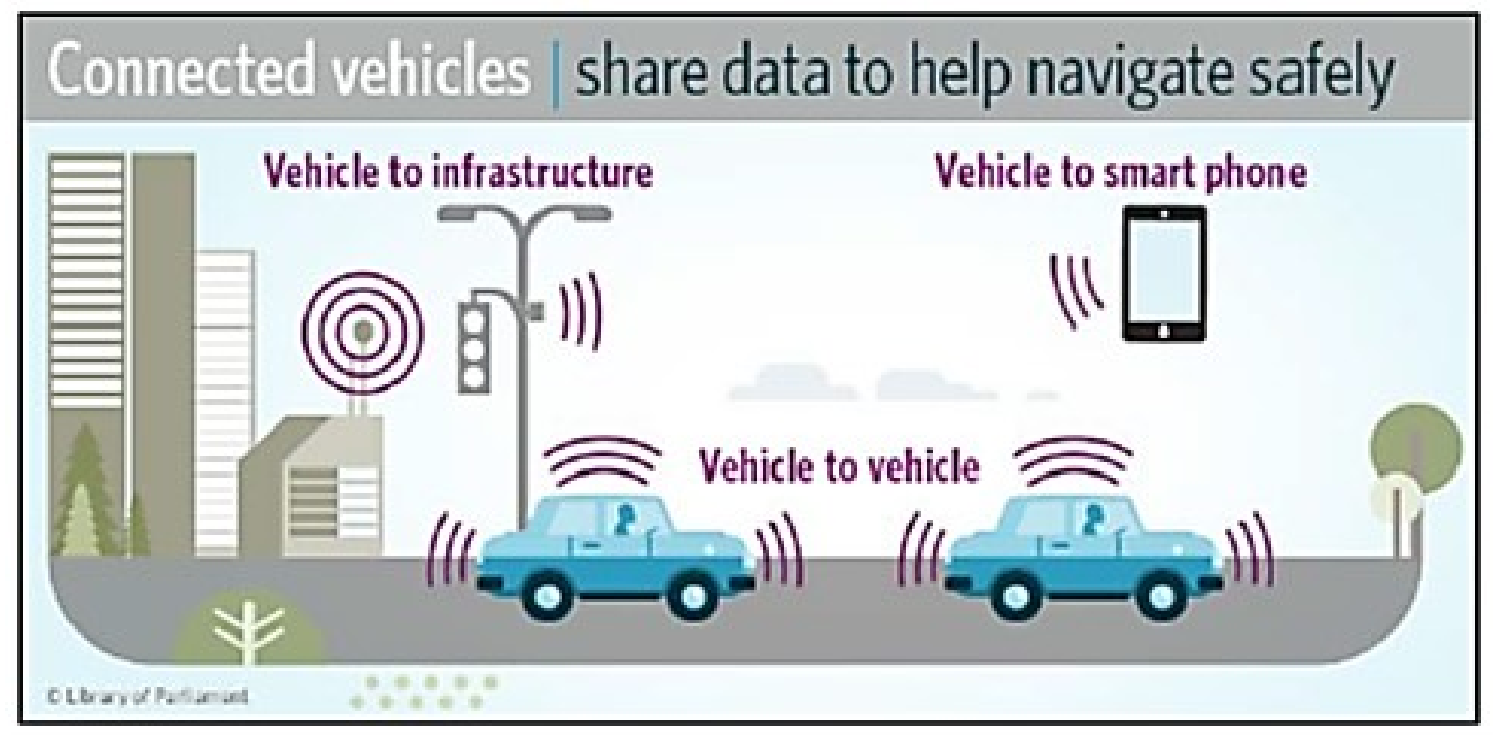

Figure 2.7: Connected Vehicles (Chong, 2016).

\section{Automated vehicles / navigate without human input}

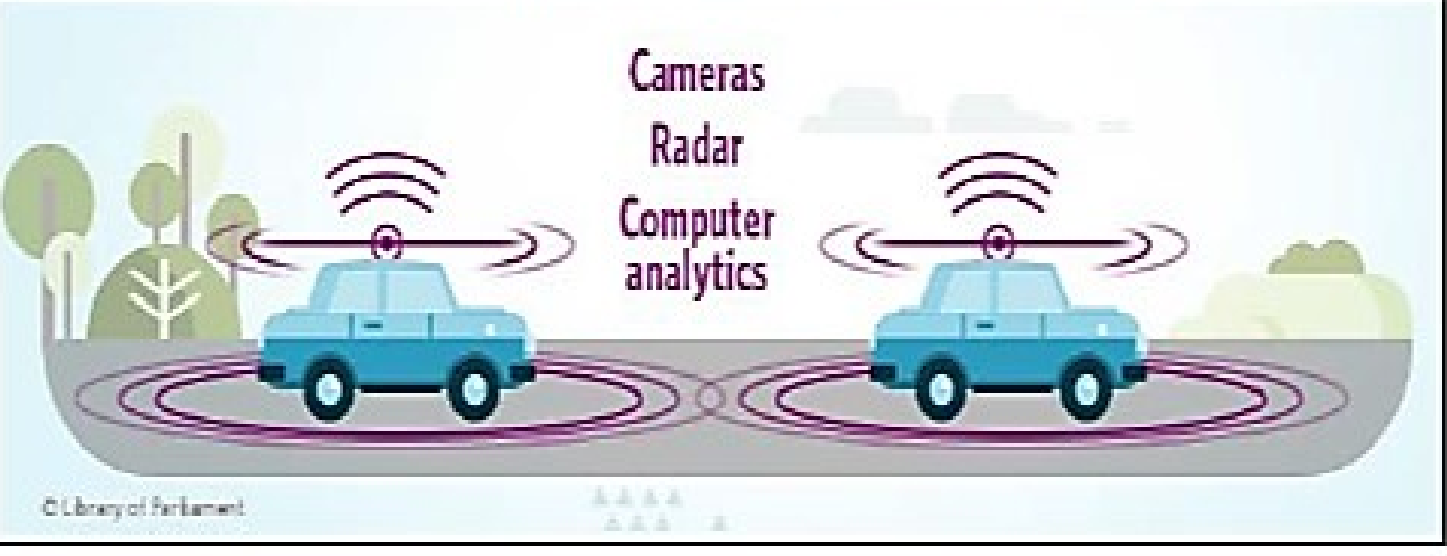

Figure 2.8: Automated Vehicles (Chong, 2016). 


\subsubsection{Benefits and Applications of Connected Vehicles}

The efforts of government, research organizations, and private sector companies to undertake the development requirements and investments for deploying the CVs are significant. Potential benefits of CV applications to make this initiative more important are safety, mobility, and environmental benefits.

\subsection{1.a Safety Benefits}

CVT applications can send warning messages to drivers about an impending collision with a vehicle travelling ahead or about a vehicle that is in the blind spot of the driver. Hill et al. (2013) stated that the CVT applications are expected to reduce the crash frequency. Figure 2.9 provides a summary of safety benefits of V2V and V2I applications such as curve speed warnings, incident warning, red-light violation warning.

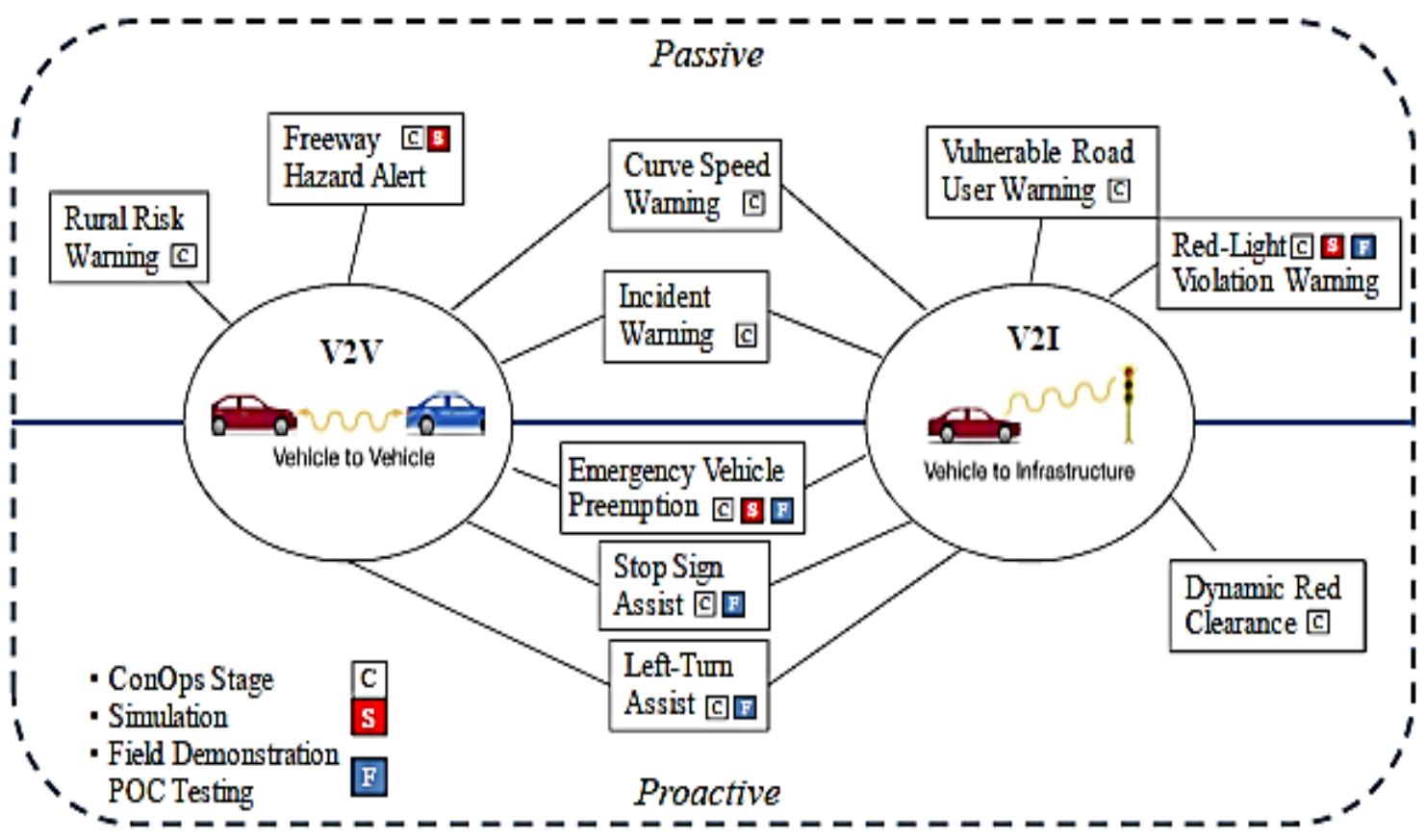

Figure 2.9: Summary of Safety Applications of CVs (Xiaosi et al., 2012). 
Where, $\mathrm{C}=$ Concepts of operations stage; $\mathrm{S}=$ Simulation; $\mathrm{F}=$ Field demonstration and proof of concept testing

The deployment of CVs and using the V2V and V2I applications either individually or together have potential to deal with 81 percentage of unimpaired driver crashes. Table 2.4 provides the details of crash frequencies that would potentially be addressed by CVT applications annually (Hill et al., 2013).

Table 2.4: Potential Benefits of CV Safety Applications (Hill et al., 2013).

\begin{tabular}{|c|c|}
\hline V2V Technology & $\begin{array}{l}\text { - } 79 \text { percentage of all vehicle crashes or } 4,409,000 \text { police- } \\
\text { reported crashes } \\
\text { - } 81 \text { percentage of light-vehicle crashes or } 4,336,000 \\
\text { police-reported crashes } \\
\text { - } 71 \text { percentage of heavy-vehicle crashes or } 267,000 \\
\text { police-reported crashes }\end{array}$ \\
\hline V2I Technology & $\begin{array}{l}\text { - } 26 \text { percentage of all vehicle crashes or } 1,465,000 \text { police- } \\
\text { reported crashes } \\
\text { - } 27 \text { percentage of light-vehicle crashes or } 1,431,000 \\
\text { police-reported crashes } \\
\text { - } 15 \text { percentage of heavy-vehicle crashes or } 55,000 \\
\text { police-reported crashes }\end{array}$ \\
\hline $\begin{array}{l}\text { Combined } \mathrm{V} 2 \mathrm{~V} \text { and } \\
\text { V2I Technologies }\end{array}$ & $\begin{array}{l}\text { - } 81 \text { percentage of all vehicle crashes or } 4,503,000 \text { police- } \\
\text { reported crashes } \\
\text { - } 83 \text { percentage of light-vehicle crashes or } 4,417,000 \\
\text { police-reported crashes } \\
\text { - } 72 \text { percentage of heavy-vehicle crashes or } 272,000 \\
\text { police-reported crashes }\end{array}$ \\
\hline
\end{tabular}




\subsection{1.b Mobility Benefits}

CV mobility applications could help drivers in efficiently navigating the roads and thus improving the function of overall transportation network. According to the Urban Mobility Report by the Texas A\&M Transportation Institute, congestion during the year 2010 in 439 American urban areas recorded 4.8 billion hours of extra time and 1.9 billion gallons of fuel wastage (Schrank, Lomax, \& Eisele, 2011). Even though there is no comprehensive analysis that provides the impacts of CVs on urban congestion, real-world data collected by CVs could be used in developing new applications to address the traffic congestion. According to Xiaosi et al. (2012), the main segments under the CV mobility application research are real-time data capture management (DCM) and dynamic mobility applications (DMA). The objective of developing DCM applications is to capture the data systematically from multiple resources for performance measurement and transportation management, whereas DMA applications are designed for the optimization of traffic and transportation operations. Figure 2.10 presents a few of the major DCM and DMA applications. 


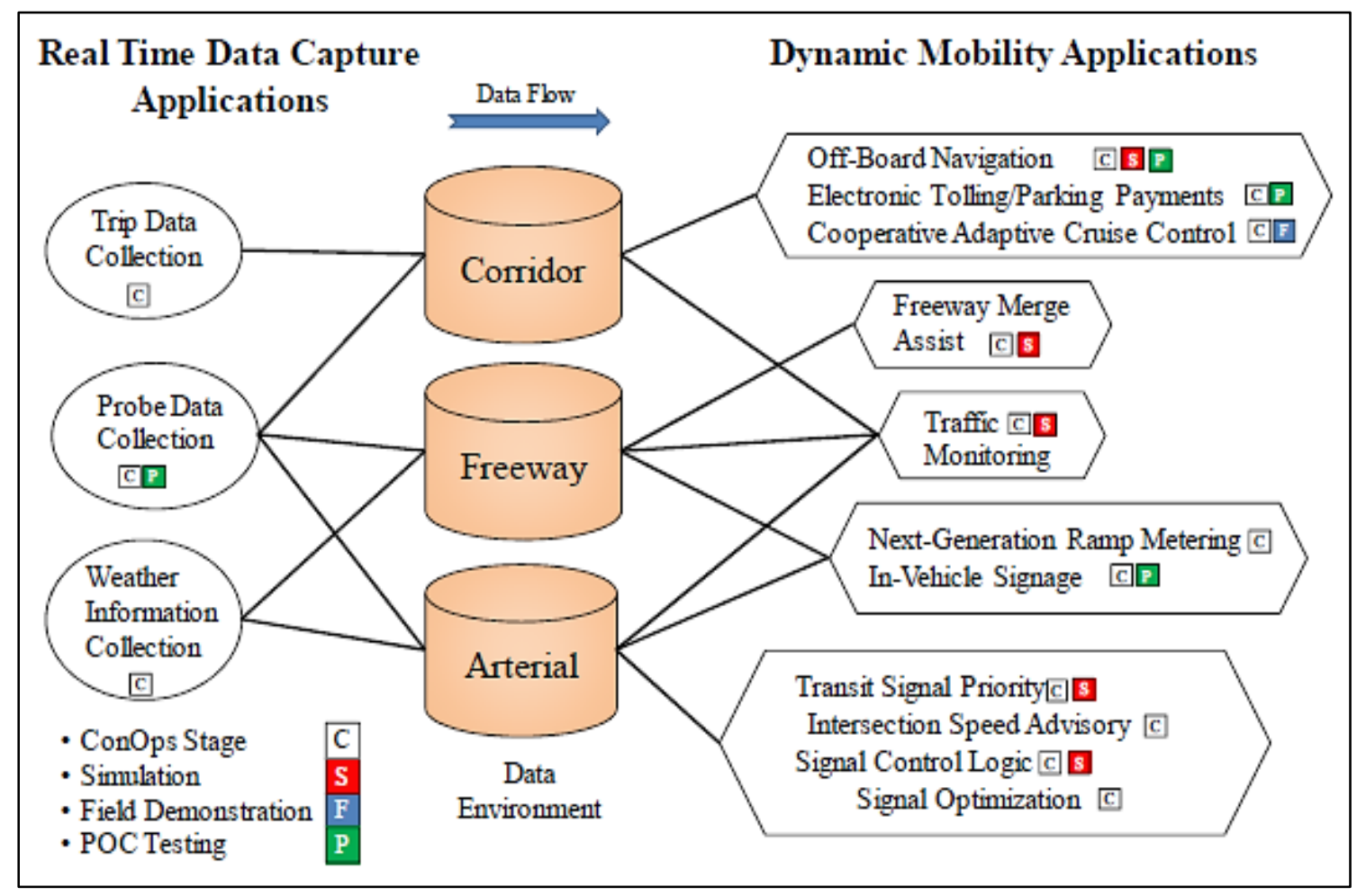

$\mathrm{C}=$ Concepts of operations stage $\mathrm{S}=$ Simulation; $\mathrm{F}=$ Field demonstration; $\mathrm{P}=$ proof of concept testing

Figure 2.10: Summary of Mobility Applications (Xiaosi et al., 2012).

\subsection{1.c Environmental Benefits}

CVs, particularly V2I applications could reduce the fuel consumption and pollutant gas emissions by reducing the traffic congestion and by improving the lane management. Since changes in weather generally have considerable impacts on traffic mobility and safety, the main focus of the road weather applications is to improve the ability to forecast weather conditions and pavement conditions more accurately. Relatively there is less research on 
the environmental applications of CVs than on safety and mobility applications (Xiaosi et al., 2012).

The City of Ottawa had partnered with Transport Canada and the Ministry of Transportation of Ontario (MTO) to demonstrate the benefits of V2I technology. The project was a one-year initiative and included a six kilometer stretch of Hunt Club Road in City of Ottawa. For this pilot project, the city provided a tablet with a system application that communicates the signal timings to the drivers and with the help of that information drivers determined the optimum speeds. After collecting the data over few months, Carleton University had analysed the economic and environmental benefits of V2I technology. It was found that there was $8.53 \%$ reduction in fuel consumption and $16.2 \%$ reduction in fuel cost, $9.47 \%$ reduction in $\mathrm{CO}_{2}$ emissions (Thompson \& Kent, 2018).

\subsubsection{Connected Vehicle Deployment Program}

The expectations of CV pilot deployment programs in the USA are to integrate the CV research concepts into practical elements and to enhance the existing operational capabilities. The objective of these programs is to encourage transit agencies, government authorities, private companies and other agencies to deploy the applications across all elements of transportation system to improve the performance (USDOT, 2018). Goals of this program are to encourage the early deployment of CVs not only through the vehicles, but also through the mobile devices and infrastructure. Also, to improve the safety, mobility and environmental benefits of CVs and to measure these benefits. Another goal of this program is to resolve the technical, institutional and financial issues of $\mathrm{CVs}$ deployment. Figure 2.11 presents the outline of the goals of CV deployment program. 


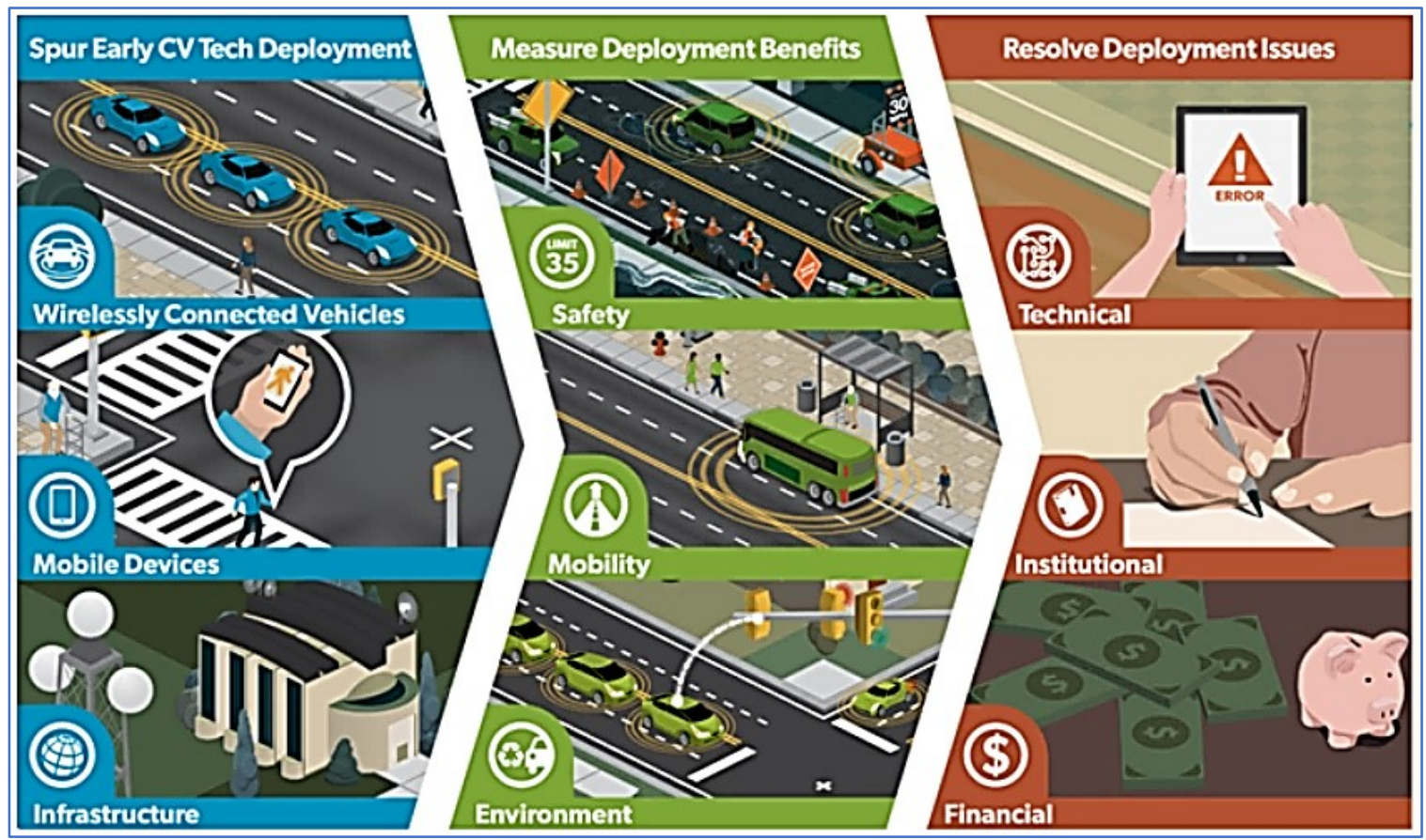

Figure 2.11: CV Pilot Deployment Program Goals (USDOT, 2018).

\subsection{2.a Safety Pilot Model Deployment - Ann Arbor}

The focus of this program was to test the CV safety applications based on V2V and V2I systems using DSRC technology to determine their effectiveness in crash frequency reduction and to ensure that the safety of the devices in not distracting the drivers. In August 2012, the deployment was launched in Ann Arbor, Michigan in over 2,800 vehicles and 29 infrastructure sites costing over \$ $\$ 50$ million. Key lessons learnt from this deployment were to develop a focused method to reach the stakeholders, to perform thorough analysis of project location, to clearly communicate the requirements to device developers and to conduct a pilot data collection test to validate the data collection, processing and assessment processes (Liz, Janet, Drennan, Mike, \& Kathy, 2018). 
The United States Department of Transportation (USDOT) is working on the regional pilot deployment programs to not only accelerate the $\mathrm{CV}$ deployment program, but also to identify the barriers in the process and how to address them. In September 2015, USDOT assigned funding of 42 million dollars to New York City Department of Transportation (NYCDOT), Wyoming Department of Transportation (WYDOT), and Tampa Hillsborough Expressway Authority (THEA) for implementing CV applications to meet their transportation needs. The selected three authorities are executing the program in three Phases and spent 12 months to prepare the deployment concept suitable for further design, testing and operation as Phase 1. In Phase 2 which is a 20-month period, sites focused on building and testing of the wireless in-vehicle, mobile devices, and roadside technologies. In Phase 3, the tested applications will be in operational practice and important performance measures will be examined and reported (Liz et al., 2018).

\subsection{2.b CV Pilot Deployment Program - New York City}

The NYCDOT aims at improving the safety of pedestrians and travelers in the city with the help of V2V and V2I applications. This pilot deployment program is anticipated to be the largest CVT deployment to date which involves approximately 5,800 cabs, 1,250 buses, 400 commercial fleet trucks, and 500 city vehicles. Using DSRC, V2I technology will be deployed at approximately 310 signalized intersections. The city will also focus on addressing vehicle-pedestrian conflicts and will include 100 pedestrians with safety devices that can assist pedestrians in safely crossing the streets. A major issue raised by stakeholders was about the privacy of the data collected. To address this issue, the program team came up with the idea of converting the latitude and longitude data to an undefined 
Cartesian coordinate system for storage and later evaluation purposes. However, other data about the vehicle movements and warning messages to drivers and their actions will still be preserved (Liz et al., 2018).

\subsection{2.c CV Pilot Deployment Program - Wyoming}

Wyoming plays an important role in moving the goods between the United states, Canada, and Mexico. Interstate 80, at 6000 feet above sea level, is a major corridor in Wyoming which moves more than 32 million tons of goods per year. During winter seasons, due to high speed winds, crash rates have been found to be 3 to 5 times higher than those during summer. WYDOT pilot program focuses on the requirements of the commercial vehicle operators and will develop applications for V2I and V2V connectivity. This program is anticipated to lower the number of truck blowover crashes and severe weather-related incidents in the Interstate 80 corridor. WYDOT will deploy approximately 75 RSUs along various sections of Interstate 80 to receive the broadcast messages. It will also equip around 400 vehicles combining fleet vehicles and heavy trucks with OBUs. Additionally, 100 WYDOT vehicles, highway patrol vehicles and snow plows will have OBUs and mobile sensors. Lessons learnt from this program so far are about including dual radios in each vehicle to listen to $\mathrm{CV}$ communication updates without the need of switching the channel (Liz et al., 2018).

\subsection{2.d CV Pilot Deployment Program - Tampa}

THEA operates the Selmon reversible express lanes which experiences high traffic volume during the peak hours resulting in urban traffic congestion. This causes considerable delay in the travel times and also a high number of rear-end and red-light running collisions. 
THEA pilot program aims at deploying V2V and V2I technologies to address the traffic congestion, collisions, and wrong entry at the REL exit. It also plans to enhance the pedestrian safety, to speed up the bus operations, and to reduce the conflicts with pedestrians at locations with high volumes of mixed traffic, using CVT. Along with the 40 RSUs, this pilot program will deploy 1,600 privately owned vehicles, 10 buses, 500 pedestrians with smartphones, and 10 trolleys. Few findings from this program so far are that the traffic signal controllers can reach their full potential only when the location and speeds of more than 90 percent of the vehicles approaching an intersection are known and the number of vehicles equipped with V2I applications would provide only a smaller percentage of vehicle coverage. After further research, THEA is installing 40 video traffic detectors at 12 intersections and will provide ten Bluetooth detectors to find out the travel time between the selected points (Liz et al., 2018).

\subsubsection{Existing Research on Freeway Merging in Connected Vehicle Environment}

Smith et al. (2012) evaluated different concepts such as lane-level variable speed limit, lane changing advisory, gap responsive on-ramp signal, and merging control in order to develop a freeway merging assistance system for CV environment. In lane-level variable speed limit task, an algorithm was developed to implement lower speed limit on the rightmost lane of the freeway based on the traffic volume densities on the left lane and the rightmost lane. It was done to create a safer merging situation by creating enough gaps for SCL vehicles to merge. It was found that the proposed algorithm could result in $6.5 \%$ increase in travel speeds, $1.1 \%$ increase in vehicle miles traveled, and $5.6 \%$ decrease in vehicle hours traveled at a network level. 
In lane changing advisory concept, an enhanced algorithm was developed to calculate the anticipated lead and lag gaps in the target lane and to provide the lane change advisory messages to the freeway through lane drivers. It creates more gaps in the freeway right most lane allowing safer merging of SCL vehicles. It was noticed that the proposed algorithm can address the freeway merging conflicts and resulted in $6.4 \%$ increase in average speed of the freeway vehicles and $5.2 \%$ decrease in the emissions. Sensitivity analysis suggested that a driver compliance rate of $90 \%$ or more for $\mathrm{CVs}$ is required to obtain the intended results from algorithm (Smith et al., 2012).

In the next task of gap responsive on-ramp signal, various algorithms were developed to estimate the time when a SCL vehicle reaches the signal at a merging point and, to determine the gap patterns available by predicting the locations of freeway main lane vehicles. An algorithm was also developed to adjust the color of the on-ramp signal based on the available gaps in freeway right lane. Results were found to be not effective due to difficulties in estimating the target time of a SCL vehicle to reach the merging point and in predicting the freeway right lane vehicle locations. However, it was found that there are some environmental benefits from this task which are $8.4 \%$ decrease in fuel consumption and $15.6 \%$ reduction in CO emissions (Smith et al., 2012).

In the merging control task, an algorithm was designed to directly control the leading and lagging vehicles on freeway mainline and the merging vehicle on SCL by completely utilizing the detailed data collected using CVT applications. Under certain assumed conditions, the results have shown $70.2 \%$ reduction in the rear-end conflicts, and $28.1 \%$ 
decrease in the lane changing conflicts and a $44.5 \%$ decrease in the total number of conflicts (Smith et al., 2012).

Smith et al. (2014) developed a prototype freeway merging control algorithm to estimate the available lead and lag gaps in freeway through lane and to select suitable strategy for controlling lead, lag and ramp vehicles to create a sufficient gap for safe merging process. In this study, microscopic traffic simulations of a freeway network in Virginia state were conducted using PTV Vissim. It was found that the proposed freeway merging algorithm can improve the road network performance. With the assumed conditions, the proposed algorithm shows a $6.3 \%$ increase in the vehicle miles travelled, $25.9 \%$ reduction in total travel times, $54.6 \%$ reduction in total delay times, $42.2 \%$ increase in average speeds, $12.0 \%$ decrease in fuel consumption, and $12.7 \%$ reduction in $\mathrm{CO}_{2}$ emissions. Sensitivity analysis indicated that a minimum $50 \%$ penetration rate of $\mathrm{CVs}$ is necessary to generate considerable benefits using the proposed algorithm and the results indicated that CVT has potential to improve the freeway operations (Smith et al., 2014).

Talebpour \& Hani (2014) conducted preliminary traffic simulations on a four-lane highway on the eastbound of Interstate-290 near Chicago, the United States, which has four onramps and three off-ramps. All the on-ramps have different merging lengths and different geometric characteristics. It was assumed that the driver's reaction time will decrease by $50 \%$ in the presence of $\mathrm{V} 2 \mathrm{~V}$ communication applications and it was observed that higher penetration rate of CVs yields in higher breakdown of traffic density. It was concluded that increase in the penetration rate of CVs increases the efficiency of freeways. 
Goodall et al. (2016) proposed an algorithm to estimate the locations of regular or noncommunicating vehicles based on the location data received from the CVs or communicating vehicles. Using car-following model the acceleration behaviour of CV's was examined and used to predict the locations of vehicles. With an assumption of $10 \%$ penetration rate for CVs, real world field data were analyzed and found that the $30 \%$ of the vehicle locations have been predicted with 9 meters accuracy in the same lane. The same algorithm was combined with an existing ramp metering algorithm and observed a significant improvement in predicting the vehicle locations.

From all the above sections, it could be noticed that there were many studies on freeway merging process considering the acceleration manners of drivers, but there were less with a focus on gap searching and acceptance behaviour at freeway entrance ramps. In the case of merging behaviour in $\mathrm{CV}$ environment, most of the studies were focused on mobility benefits of CVs. Even though some studies focused on safety benefits of CVs, the results did not quantify the safety benefits in terms of reduction in collision frequency. Those studies found that safety applications of CVs could reduce the conflicts in the merging area. Hence, the current study attempts to determine the changes in PNC in the CV environment and thus relating the $\mathrm{PNC}$ to the reduction in collision frequency on acceleration lanes with the advantages of CVT. 


\section{Chapter 3: DATA COLLECTION AND PRELIMINARY ANALYSIS}

This study is an extension of the past research projects that were executed at Carleton University. Sarhan (2004) studied safety performance of 59 merge and 39 diverge areas on Highway 417 along the City of Ottawa. Speed data on 23 acceleration SCL sites of the Highway 417 were collected by Ahammed et al. (2008) and driver's behaviour at the merge areas was examined. Salehi (2010) performed a study using reliability-based approaches to analyze the sufficiency of the SCL length for the acceleration lanes. Fatema \& Hassan (2013) presented a reliability-based design method for the SCL length by studying the acceleration and gap acceptance behaviour of drivers. The present study considers these previous studies as a reference to develop new methodologies for improving the safety of the entrance freeway ramps by considering the benefits of vehicle connectivity. Field data collected by Ahammed et al., (2008) were received along with the processed data of acceleration and merging distance, which were used as base data to achieve the objectives of this study.

This chapter provides the overview of selecting the input parameters, statistics of the parameters and the primary analysis of the input parameters. Section 3.1 explains the process of site selection and geometrical properties of the selected sites. Section 3.2 provides the description of the data collected by Ahammed (2005). Section 3.3 describes the traffic volume data on freeway second lane (F2L) whereas Section 3.4 provides the description of the normality tests on the input parameters. 


\subsection{Site Selection}

Highway 417 is the major highway passing through the heart of the City of Ottawa and has a posted speed limit of $100 \mathrm{~km} / \mathrm{h}$. It is an important part of the Trans-Canada highway across the eastern Ontario with a total length of $186 \mathrm{~km}$. In the City of Ottawa, the number of lanes varies from six to eight between Highway 174 to Highway 416. The selected section of Highway 417 consists of acceleration lanes with varying geometric characteristics. Figure 3.1 shows the image of the study road, and the chosen SCL sites were marked with a star symbol.

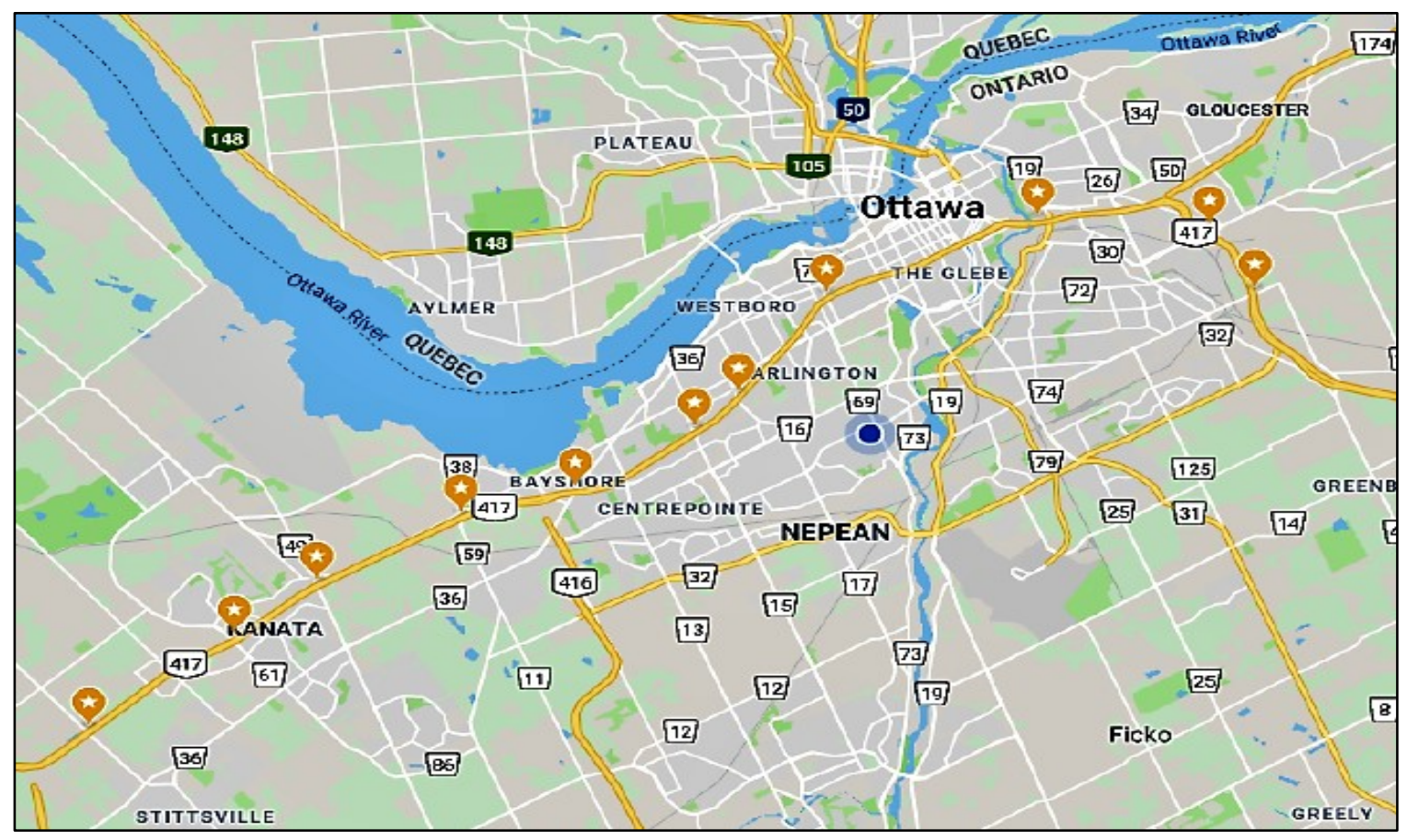

Figure 3.1: Image of the Study Road (Google Maps).

The selected study area contains 66 entrance SCLs and 27 exit SCLs from which only entrance SCLs were considered in this study. Ahammed et al., (2008) collected the speed 
data of SCL vehicles and FRL vehicles using laser speed guns. After an initial assessment, only 40 acceleration SCLs were considered for the data collection. Because of the refusals from the MTO and other limitations to the process, data collection was limited to 23 SCLs. The equipment was setup by Ahammed et al., (2008), at various available positions like upper floors of buildings, roofs or overpasses. Amongst the final 23 SCLs, 14 were located in the eastbound direction and 9 were located in the westbound direction. Based on the location of data collection, SCLs were classified as full profile or merge speed only sites. For the full profile sites, speed data was collected from the gore location to the merging point. Partial speed profiles were collected for the merge speed only sites from which only merge speed can be obtained. Among 23 sites, 16 were limited-length type and seven were extended type. For an extended type SCL, the length of acceleration lane was measured from a point where the freeway right lane and the edges of the ramp pavement at the entrance gore are $1.25 \mathrm{~m}$ apart to the point where the exit gore and the freeway lane are $1.25 \mathrm{~m}$ apart and to the end of the taper for a limited-length type SCL. The lengths of extended type SCLs varied from $673 \mathrm{~m}$ to $1366 \mathrm{~m}$ whereas for limited-length type, lengths varied from $188 \mathrm{~m}$ to $468 \mathrm{~m}$. Table 3.1 presents the list of SCL sites. 
Table 3.1: List of SCL Sites (Ahammed, 2005).

\begin{tabular}{|c|c|c|c|c|}
\hline $\begin{array}{c}\text { Interchange } \\
\text { (IC) } \\
\text { Number }\end{array}$ & Direction & Site Name & Profile Type & SCL Type \\
\hline 110 & West & Walkley W-W & Merge Speed Only & Limited \\
\hline 112 & East & Innes E-E & Merge Speed Only & Limited \\
\hline 115 & West & St. Laurent Blvd N-W & Full Profile & Extended \\
\hline 117 & West & Vanier N-W & Full Profile & Extended \\
\hline 117 & East & Vanier N-E & Merge Speed Only & Limited \\
\hline 118 & East & Nicholas N-E & Merge Speed Only & Extended \\
\hline 122 & West & Parkdale NS-W & Full Profile & Limited \\
\hline 126 & East & Maitland NS-W & Full Profile & Limited \\
\hline 127 & West & Woodroffe NS-W & Full Profile & Limited \\
\hline 127 & East & Woodroffe N-E & Merge Speed Only & Limited \\
\hline 127 & East & Woodroffe S-E & Merge Speed Only & Extended \\
\hline 129 & East & Greenbank S-E & Merge Speed Only & Extended \\
\hline 130 & East & Richmond S-E & Full Profile & Limited \\
\hline 134 & West & Moodie N-W & Full Profile & Limited \\
\hline 138 & West & Eagleson N-W & Merge Speed Only & Extended \\
\hline 138 & East & Eagleson S-E & Full Profile & Limited \\
\hline 139 & East & Castlefrank NS-E & Full Profile & Extended \\
\hline 140 & West & Terryfox N-W & Full Profile & Limited \\
\hline 140 & West & Terryfox S-W & Merge Speed Only & Limited \\
\hline 140 & East & Terryfox N-E & Merge Speed Only & Limited \\
\hline 140 & East & Terryfox S-E & Merge Speed Only & Limited \\
\hline 144 & East & Carp N-E & Merge Speed Only & Limited \\
\hline 144 & East & Carp S-E & Full Profile & Limited \\
\hline
\end{tabular}

Due to the tapered end of a limited-length type SCL, drivers would have less time and lower acceleration lane lengths to complete the merging action when compared with an extended type SCL. Extended type SCL provides more length for acceleration and more time to perform the merge operation which felicitates a relatively easy merging process compared to a limited-length type SCL. To get a clear understanding of the typical merging behaviour of SCL vehicles when they have limited time and distance for accelerating from gore to merging point, only the 16 limited-length type sites were considered for further 
process, and the extended type SCL's were excluded from this study. Geometric features of the selected 16 sites are listed in Table 3.2.

Table 3.2: Geometry of the SCL Sites (Ahammed, 2005).

\begin{tabular}{|l|c|c|c|c|c|c|}
\hline \multicolumn{1}{|c|}{ Site name } & $\begin{array}{c}\text { Ramp } \\
\text { Type }\end{array}$ & $\begin{array}{c}\text { SCL } \\
\text { Type }\end{array}$ & $\begin{array}{c}\text { Ramp } \\
\text { Grade }\end{array}$ & $\begin{array}{c}\text { SCL } \\
\text { Grade }\end{array}$ & $\begin{array}{c}\text { SCL } \\
\text { Length (m) }\end{array}$ & $\begin{array}{c}\text { Number of } \\
\text { Lanes of } \\
\text { Freeway }\end{array}$ \\
\hline Carp N-E & Loop & Parallel & Down & Flat & 425 & 2 \\
\hline Carp S-E & Outer & Parallel & Down & Flat & 430 & 2 \\
\hline Eagleson S-E & Outer & Parallel & Down & Up & 327 & 2 \\
\hline Innes E-E & Loop & Parallel & Down & Flat & 354 & 2 \\
\hline Maitland NS-W & Outer & Parallel & Down & Flat & 468 & 3 \\
\hline Moodie N-W & Outer & Parallel & Down & Up & 323 & 2 \\
\hline Parkdale NS-W & Outer & Taper & Up & Up & 188 & 4 \\
\hline Richmond S-E & Outer & Parallel & Down & Flat & 321 & 4 \\
\hline Terryfox N-E & Loop & Parallel & Down & Flat & 418 & 2 \\
\hline Terryfox N-W & Outer & Parallel & Down & Flat & 419 & 2 \\
\hline Terryfox S-E & Outer & Parallel & Down & Flat & 437 & 2 \\
\hline Terryfox S-W & Loop & Parallel & Down & Flat & 395 & 2 \\
\hline Vanier N-E & Loop & Parallel & Down & Flat & 363 & 3 \\
\hline Walkley W_W & Loop & Parallel & Down & Flat & 422 & 2 \\
\hline Woodroffe N-E & Loop & Parallel & Down & Flat & 248 & 3 \\
\hline Woodroffe NS-W & Outer & Parallel & Down & Flat & 346 & 3 \\
\hline
\end{tabular}

From Table 3.2, it can also be noticed that, seven out of 16 sites are loop type ramps and the rest are outer type ramps. There is only one site with the tapered SCL and the remaining are parallel type SCLs. All ramps are on down grade except for one site with an up-grade. The grade of all SCLs is flat except for three SCLs with an up-grade. The length of SCL of the 16 sites varied from $188 \mathrm{~m}$ to $468 \mathrm{~m}$. Eight of the 16 sites have their lengths between $200 \mathrm{~m}$ and $400 \mathrm{~m}$. There is only one site with the length less than $200 \mathrm{~m}$ and there are seven sites with lengths above $400 \mathrm{~m}$. Number of lanes of the freeway varies from two to four in 
each travel direction. While most of the sites have two lanes, there are two sites with four lanes and there are four sites with three lanes in each direction of travel.

\subsection{Data Collection}

This section provides the description of different field data like gore speed, merge speed, acceleration rate, traffic volumes on FRL and SCL, and collision data which were received from Ahammed et al., (2008). It also provides the details of traffic videos received by the author of this study from the MTO.

\subsubsection{Gore Speed}

The initial speed of SCL vehicle at the gore location is referred to as gore speed in this study. Gore speeds of SCL vehicles were collected for only full profile sites. For the merge speed only profile sites, gore speed was calculated by Fatema \& Hassan (2013) using the recorded merge speeds, which was explained in the Chapter 4. Gore speed data for the eight full profile sites includes: mean gore speed, standard deviation of gore speed and $85^{\text {th }}$ percentile gore speed. As shown in Table 3.3, the mean gore speed of the SCL drivers varied from 62.51 to $100.05 \mathrm{~km} / \mathrm{h}$ whereas the standard deviation varied from 5.0 to 9.48 $\mathrm{km} / \mathrm{h}$. The $85^{\text {th }}$ percentile gore speed varied from 67.09 to $109.06 \mathrm{~km} / \mathrm{h}$. A graphical distribution of the mean, $85^{\text {th }}$ percentile and standard deviation of gore speeds at the full profile sites are presented in Figure 3.2. 
Table 3.3: Gore Speeds at Study SCL Sites.

\begin{tabular}{|l|c|c|c|}
\hline \multirow{2}{*}{\multicolumn{1}{c|}{ SCL Site }} & \multicolumn{3}{|c|}{ Gore Speed (km/h) } \\
\cline { 2 - 4 } & Mean & Standard Deviation & 85th Percentile \\
\hline Carp S-E & 99.85 & 7.62 & 108.05 \\
\hline Eagleson S-E & 95.71 & 9.43 & 105.72 \\
\hline Maitland NS-W & 62.51 & 5.03 & 67.09 \\
\hline Moodie N-W & 91.77 & 8.69 & 101.03 \\
\hline Parkdale NS-W & 69.73 & 9.12 & 77.98 \\
\hline Richmond S-E & 83.28 & 7.85 & 91.72 \\
\hline Terryfox N-W & 100.05 & 9.48 & 109.06 \\
\hline Woodroffe NS-W & 78.85 & 8.00 & 86.55 \\
\hline
\end{tabular}

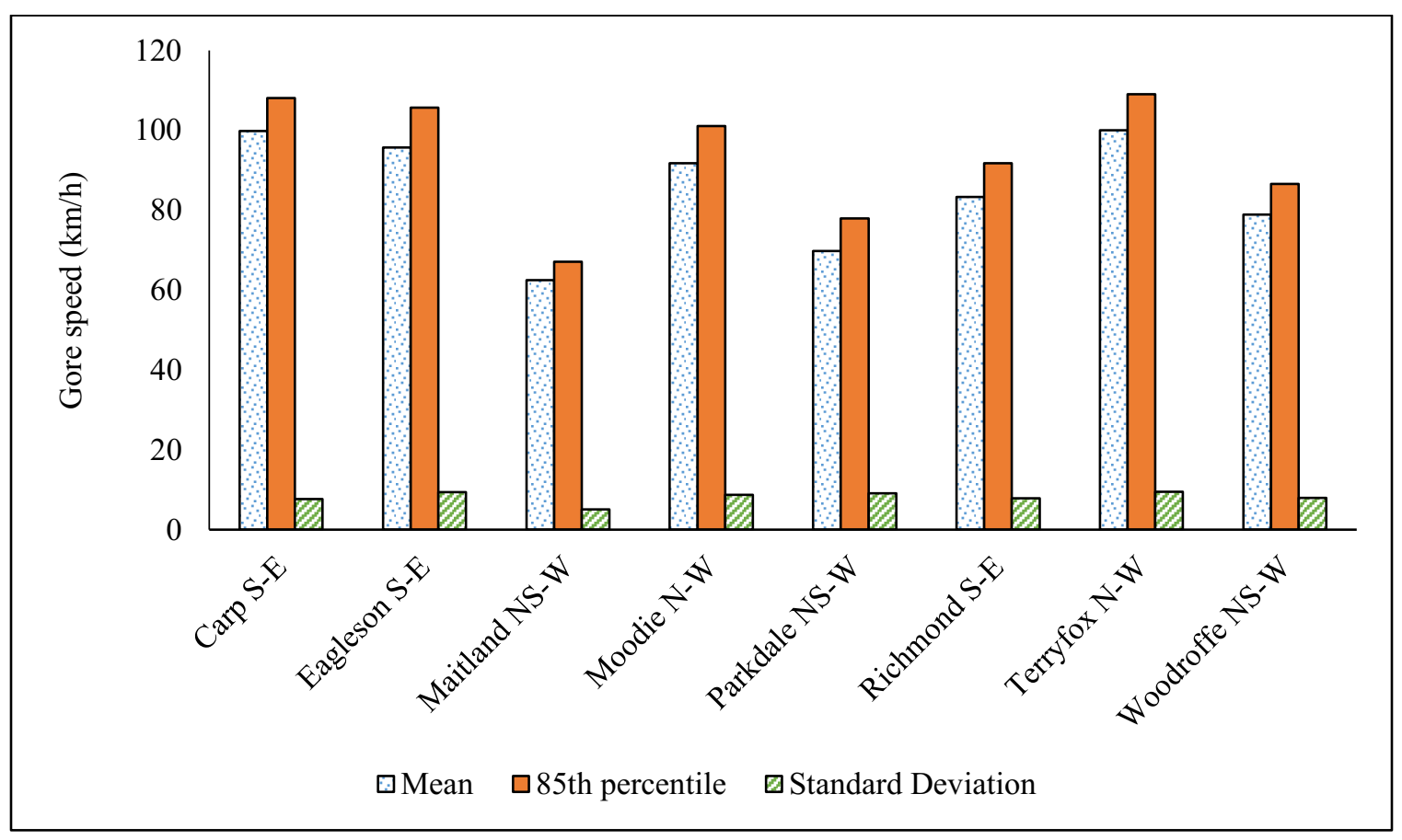

Figure 3.2: Distribution of Gore Speeds. 


\subsubsection{Merge Speed}

Speed of the SCL vehicles at the point of merge is termed as merge speed and this data was available for all 16 SCL sites. As shown in Table 3.4, mean merge speed varies from 80.12 to $105.95 \mathrm{~km} / \mathrm{h}$ and the standard deviation varies from 6.96 to $11.16 \mathrm{~km} / \mathrm{h}$. The range of $85^{\text {th }}$ percentile of merge speed was from 93.40 to $114.76 \mathrm{~km} / \mathrm{h}$. Graphical distributions of the mean, $85^{\text {th }}$ percentile and standard deviation of merge speed at all the selected SCL sites are displayed in Figure 3.3.

Table 3.4: Merge Speeds at Study SCL Sites.

\begin{tabular}{|l|c|c|c|}
\hline \multirow{2}{*}{\multicolumn{1}{|c|}{ SCL Site }} & \multicolumn{3}{|c|}{ Merge Speed (km/h) } \\
\cline { 2 - 4 } & Mean & Standard Deviation & 85th Percentile \\
\hline Carp N-E & 91.26 & 11.16 & 102.60 \\
\hline Carp S-E & 105.95 & 6.96 & 112.30 \\
\hline Eagleson S-E & 98.54 & 9.32 & 108.31 \\
\hline Innes E-E & 90.87 & 10.03 & 100.26 \\
\hline Maitland NS-W & 86.57 & 9.74 & 96.56 \\
\hline Moodie N-W & 93.16 & 8.78 & 102.21 \\
\hline Parkdale NS-W & 80.12 & 10.09 & 90.51 \\
\hline Richmond S-E & 87.68 & 8.44 & 96.41 \\
\hline Terryfox N-E & 89.93 & 10.36 & 100.00 \\
\hline Terryfox N-W & 104.71 & 9.94 & 114.76 \\
\hline Terryfox S-E & 102.6 & 10.31 & 114.6 \\
\hline Terryfox S-W & 92.6 & 10.80 & 102.0 \\
\hline Vanier N-E & 86.2 & 7.12 & 93.40 \\
\hline Walkley W-W & 83.9 & 10.64 & 95.20 \\
\hline Woodroffe N-E & 87.5 & 7.40 & 94.90 \\
\hline Woodroffe NS-W & 88.50 & 9.35 & 98.62 \\
\hline
\end{tabular}




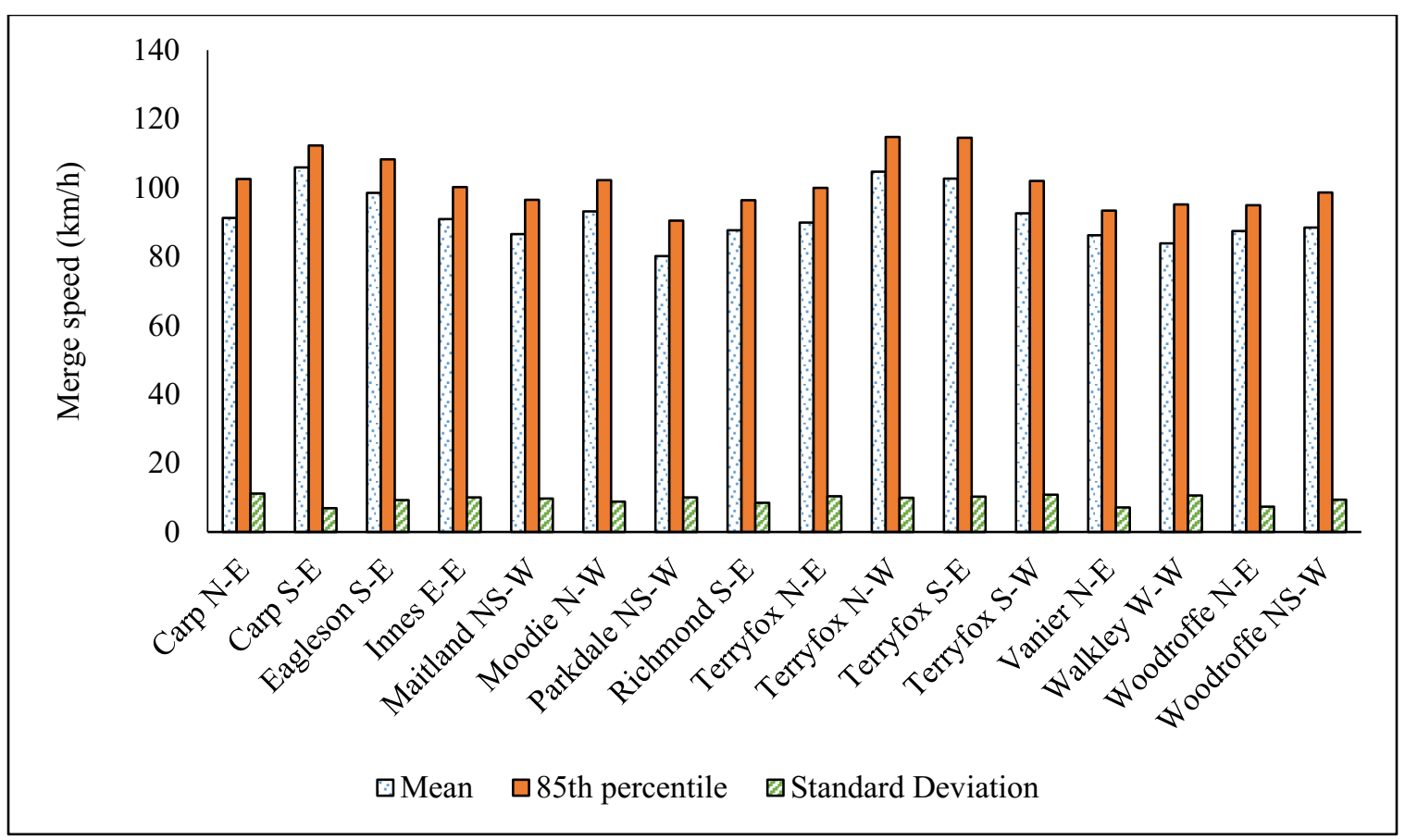

Figure 3.3: Distribution of Merge Speeds.

\subsubsection{FRL Vehicle Speed}

Speeds of the vehicles on the right most lane of the freeway at all $16 \mathrm{SCL}$ sites were collected downstream the gore. As shown in Table 3.5, mean speed of FRL vehicles varied from 90.4 to $109.29 \mathrm{~km} / \mathrm{h}$ and the range of standard deviation was between 7.29 and 11.75 $\mathrm{km} / \mathrm{h}$. The range of $85^{\text {th }}$ percentile speed of FRL vehicles was from 98.9 to $117.54 \mathrm{~km} / \mathrm{h}$. The variation of mean, $85^{\text {th }}$ percentile and standard deviation of FRL vehicle speeds among the SCL sites are represented by Figure 3.4. 
Table 3.5: Freeway Speeds at SCL Sites.

\begin{tabular}{|l|c|c|c|}
\hline \multirow{2}{*}{\multicolumn{1}{|c|}{ SCL Site }} & \multicolumn{3}{|c|}{ Freeway Speed (km/h) } \\
\cline { 2 - 4 } & Mean & Standard Deviation & 85th Percentile \\
\hline Carp N-E & 109.73 & 8.17 & 117.10 \\
\hline Carp S-E & 109.29 & 7.52 & 117.54 \\
\hline Eagleson S-E & 98.29 & 7.90 & 106.87 \\
\hline Innes E-E & 106.31 & 7.29 & 112.44 \\
\hline Maitland NS-W & 96.64 & 8.65 & 106.39 \\
\hline Moodie N-W & 97.80 & 7.85 & 106.52 \\
\hline Parkdale NS-W & 98.49 & 11.75 & 110.67 \\
\hline Richmond S-E & 98.99 & 10.30 & 109.78 \\
\hline Terryfox N-E & 103.43 & 8.18 & 111.92 \\
\hline Terryfox N-W & 108.27 & 7.51 & 116.23 \\
\hline Terryfox S-E & 104.20 & 8.91 & 113.60 \\
\hline Terryfox S-W & 107.60 & 8.40 & 115.50 \\
\hline Vanier N-E & 90.40 & 8.11 & 98.90 \\
\hline Walkley W-W & 106.10 & 10.84 & 116.50 \\
\hline Woodroffe N-E & 96.10 & 8.62 & 105.20 \\
\hline Woodroffe NS-W & 102.87 & 10.42 & 115.98 \\
\hline
\end{tabular}

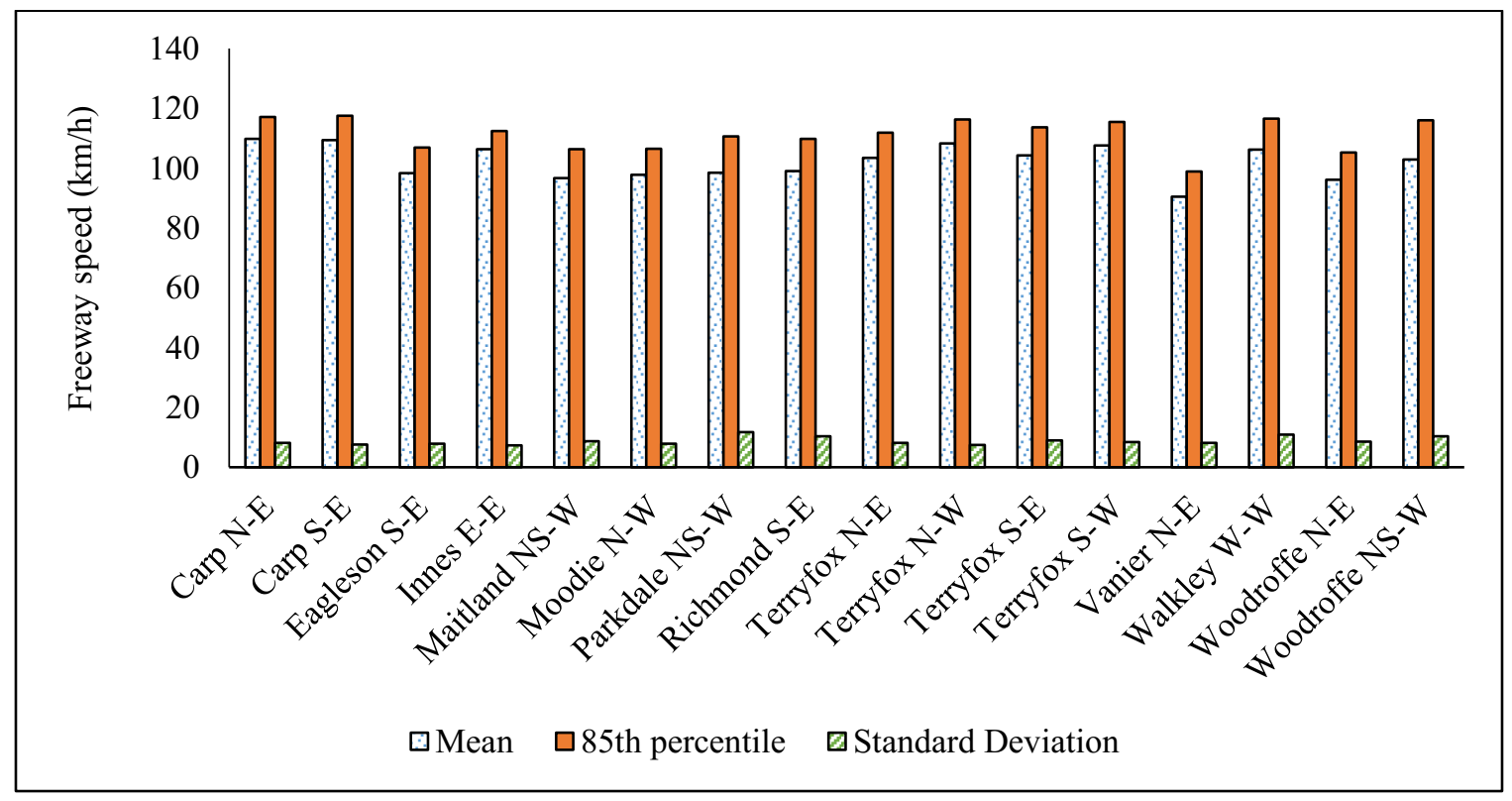

Figure 3.4: Distribution of Freeway Speeds. 


\subsubsection{Acceleration Rate}

Along with the speed data, Ahammed et al. (2008) recorded the time and distances data and calculated the mean acceleration rate, maximum acceleration rate and the overall acceleration rate of SCL vehicles. Mean acceleration rate was obtained by calculating the mean of all acceleration rates whereas the maximum acceleration rate refers to the maximum of the calculated acceleration values. On the other hand, overall acceleration rate was calculated using the time taken to accelerate from the gore speed to merge speed and speeds at the beginning of SCL length and merging point. After observing the mean and maximum acceleration rates recorded while varying the speed interval and time interval between the recordings, Ahammed (2005) considered overall acceleration rate as the accurate indicator for vehicle acceleration rates. In this study overall acceleration rate was considered with an understanding that it could capture the SCL vehicle's acceleration behaviour from the initial point to the merging point. Hassan et al., (2012) and Fatema \& Hassan (2013) also considered the overall acceleration rate in the past research works. In this study, overall acceleration rate is referred as acceleration rate in the further chapters.

Acceleration rates data was available for only full profile sites. As shown in Table 3.6, mean overall acceleration rate of the full profile sites varied between $0.100 \mathrm{~m} / \mathrm{s}^{2}$ and 0.843 $\mathrm{m} / \mathrm{s}^{2}$ and the standard deviation ranged from 0.199 to $0.291 \mathrm{~m} / \mathrm{s}^{2}$. The range of $85^{\text {th }}$ percentile of acceleration rate was between $0.316 \mathrm{~m} / \mathrm{s}^{2}$ and $1.120 \mathrm{~m} / \mathrm{s}^{2}$. Distributions of mean, $85^{\text {th }}$ percentile and standard deviation of acceleration rates are represented by Figure 3.5 . 
Table 3.6: Acceleration Rates at SCL Sites.

\begin{tabular}{|l|c|c|c|}
\hline \multirow{2}{*}{\multicolumn{1}{|c|}{ SCL Site }} & \multicolumn{3}{c|}{ Acceleration Rate $\left(\mathbf{m} / \mathbf{s}^{\mathbf{2}}\right)$} \\
\cline { 2 - 4 } & Mean & Standard Deviation & $\mathbf{8 5 t h}$ Percentile \\
\hline Carp S-E & 0.228 & 0.199 & 0.402 \\
\hline Eagleson S-E & 0.232 & 0.216 & 0.316 \\
\hline Maitland NS-W & 0.843 & 0.255 & 1.120 \\
\hline Moodie N-W & 0.100 & 0.224 & 0.327 \\
\hline Parkdale NS-W & 0.715 & 0.288 & 0.969 \\
\hline Richmond S-E & 0.284 & 0.226 & 0.505 \\
\hline Terryfox N-W & 0.221 & 0.206 & 0.416 \\
\hline Woodroffe NS-W & 0.649 & 0.291 & 0.860 \\
\hline
\end{tabular}

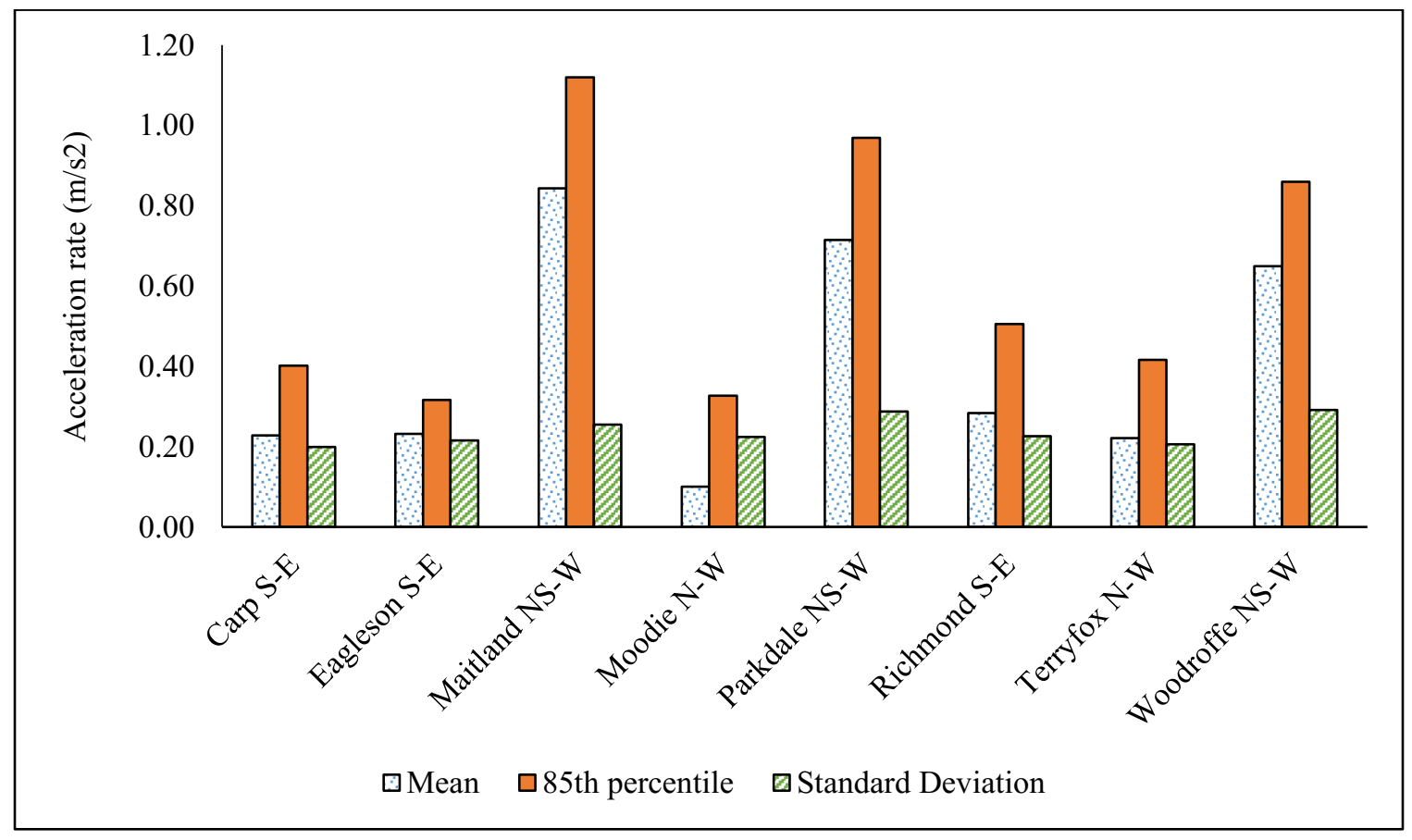

Figure 3.5: Distribution of Overall Acceleration Rates. 


\subsubsection{Traffic Volumes on FRL and SCL}

Traffic volumes on FRL and SCL were recorded by Ahammed (2005) using a video camera at each site and the captured videos were processed to obtain the hourly traffic volumes on FRL and SCL. Percentages of heavy vehicles (HV) and passenger cars (PC) on FRL were also obtained from the video recordings. As shown in Table 3.7, hourly traffic volumes at the sixteen SCL sites varied from $345 \mathrm{veh} / \mathrm{h}$ to $1257 \mathrm{veh} / \mathrm{h}$ on FRL and from $95 \mathrm{veh} / \mathrm{h}$ to $965 \mathrm{veh} / \mathrm{h}$ on SCL. Percentage of HV on FRL varied from $5.1 \%$ to $20.2 \%$ and from 0.0 to $8.8 \%$ on SCL. Hourly traffic volumes on FRL and SCL are shown in Figure 3.6 and the percentages of HV and PC are shown in Figure 3.7 and Figure 3.8.

Table 3.7: Traffic Volumes on FRL and SCL.

\begin{tabular}{|c|c|c|c|c|c|c|}
\hline SCL Site & $\begin{array}{c}\text { FRL } \\
\text { Volume } \\
\text { (veh/h) }\end{array}$ & $\begin{array}{c}\text { HV on } \\
\text { FRL } \\
(\%)\end{array}$ & $\begin{array}{c}\text { PC on } \\
\text { FRL } \\
(\%)\end{array}$ & $\begin{array}{c}\text { SCL } \\
\text { Volume } \\
\text { (veh/h) }\end{array}$ & $\begin{array}{l}\text { HV on } \\
\text { SCL } \\
(\%)\end{array}$ & $\begin{array}{l}\text { PC on } \\
\text { SCL } \\
(\%)\end{array}$ \\
\hline Carp N-E & 521 & 7.4 & 92.6 & 193 & 4.8 & 95.2 \\
\hline Carp S-E & 709 & 16.3 & 83.7 & 271 & 6.1 & 93.9 \\
\hline Eagleson S-E & 1257 & 11.0 & 89.0 & 429 & 3.2 & 96.8 \\
\hline Innes E-E & 848 & 18.8 & 81.2 & 237 & 5.8 & 94.2 \\
\hline Maitland NS-W & 1229 & 11.1 & 88.9 & 594 & 2.0 & 98.0 \\
\hline Moodie N-W & 1244 & 11.8 & 88.2 & 111 & 4.9 & 95.1 \\
\hline Parkdale NS-W & 588 & 5.1 & 94.9 & 422 & 3.5 & 96.5 \\
\hline Richmond S-E & 492 & 6.1 & 93.9 & 437 & 6.0 & 94.0 \\
\hline Terryfox N-E & 685 & 18.7 & 81.3 & 188 & 4.3 & 95.7 \\
\hline Terryfox N-W & 661 & 20.2 & 79.8 & 161 & 6.0 & 94.0 \\
\hline Terryfox S-E & 785 & 15.0 & 85.0 & 351 & 6.7 & 93.3 \\
\hline Terryfox S-W & 704 & 20.0 & 80.0 & 95 & 8.8 & 91.2 \\
\hline Vanier N-E & 1195 & 13.4 & 86.6 & 294 & 1.8 & 98.2 \\
\hline Walkley W-W & 345 & 13.6 & 86.4 & 965 & 6.3 & 93.7 \\
\hline Woodroffe N-E & 966 & 11.0 & 89.0 & 191 & 0.0 & 100.0 \\
\hline Woodroffe NS-W & 886 & 11.5 & 88.5 & 354 & 4.0 & 96.0 \\
\hline \multicolumn{7}{|c|}{$\begin{array}{l}\mathrm{SCL}=\text { Speed Change Lane; FRL }=\text { Freeway Right Lane; } \mathrm{HV}=\text { Heavy Vehicles; } \mathrm{PC}=\text { Passenge } \\
\text { Cars }\end{array}$} \\
\hline
\end{tabular}




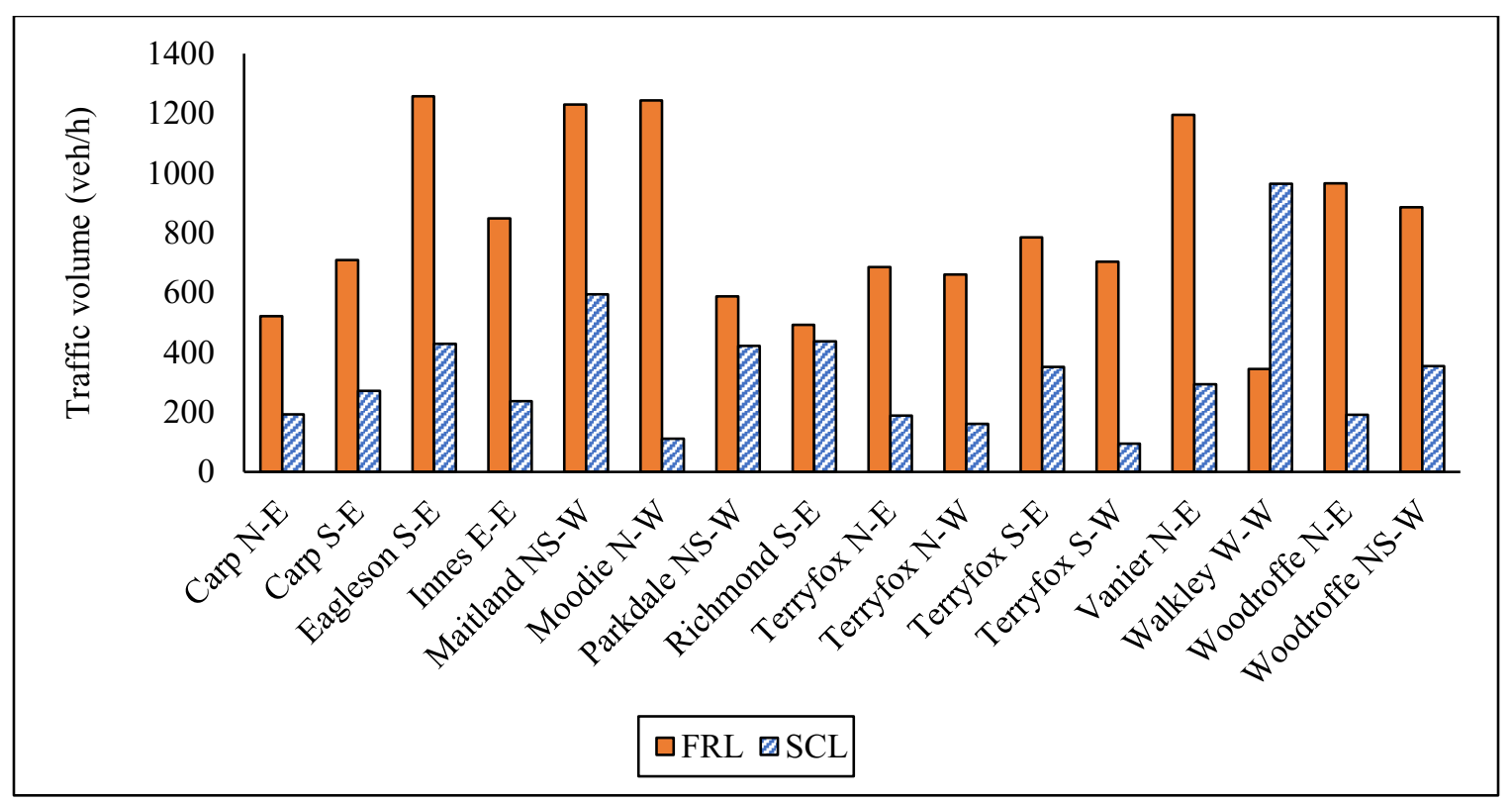

Figure 3.6: Traffic Volume on FRL and SCL.

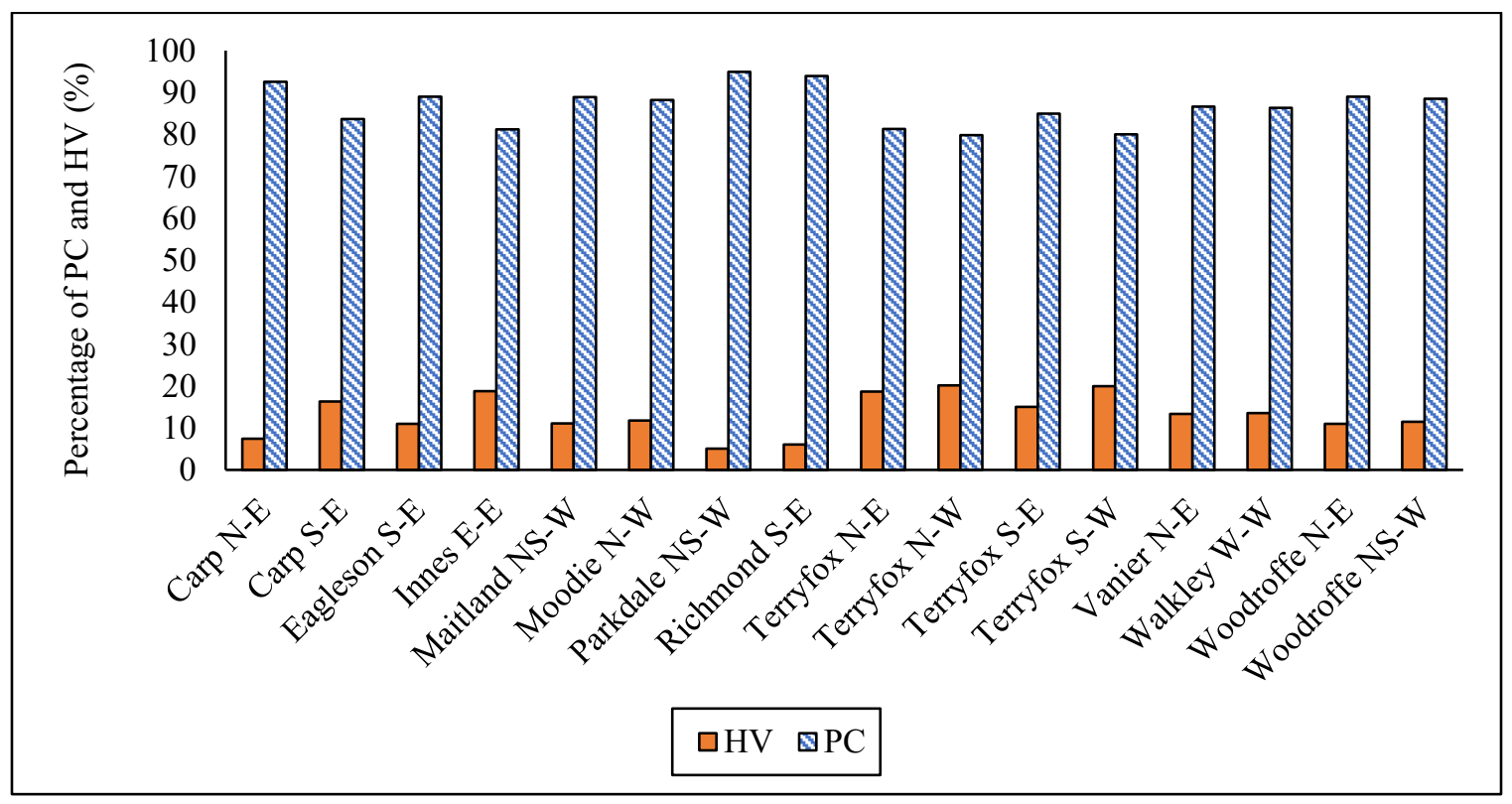

Figure 3.7: Percentage of PC and HV on FRL. 


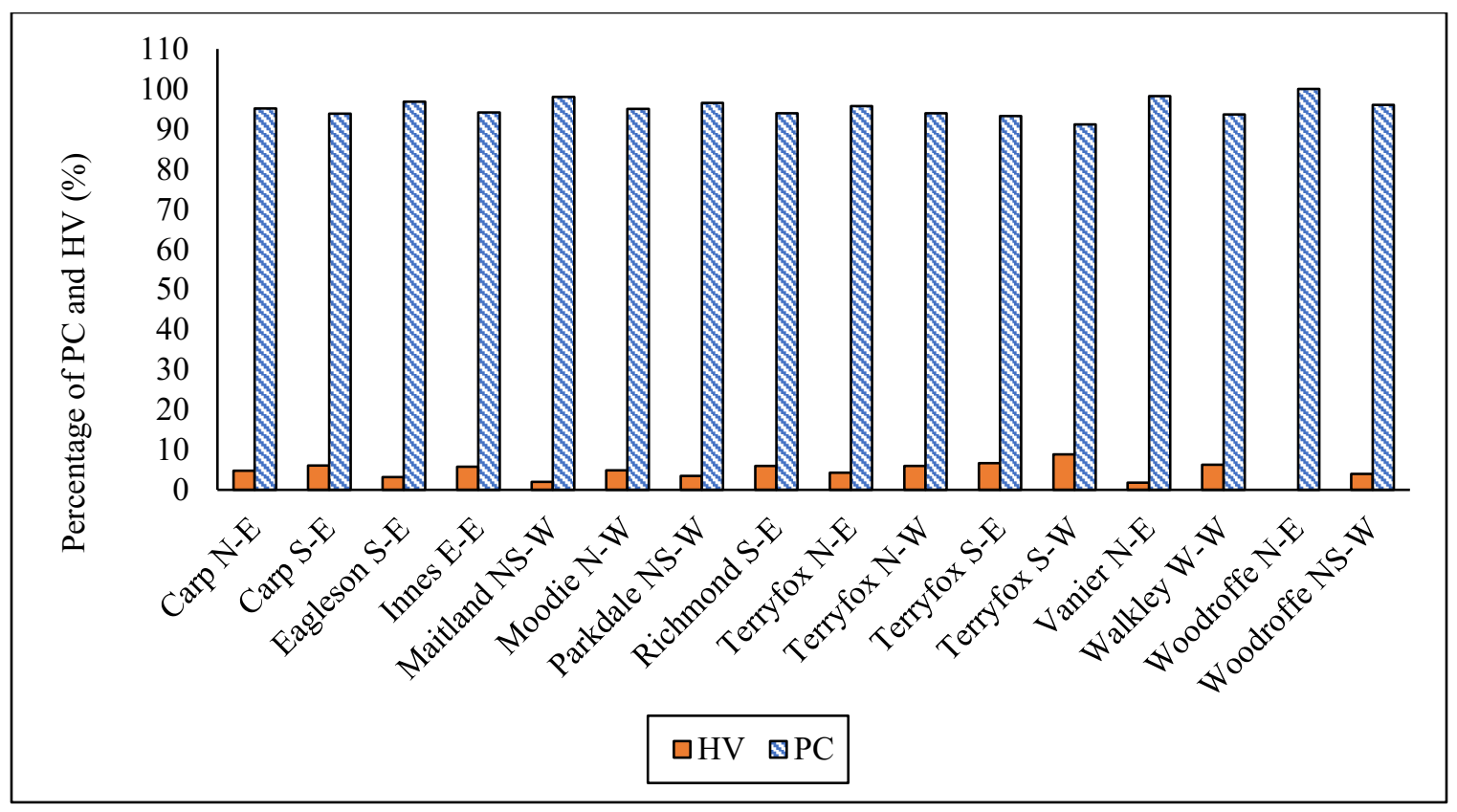

Figure 3.8: Percentage of PC and HV on SCL.

\subsubsection{Collision Data}

The author of this study received the collision data from Ahammed (2005) in the form of a MS Excel file. This file has the count of total collisions for a period of five years from 1998 to 2002 on Highway 417 within City of Ottawa. Based on severity, collisions were categorized as fatal, injury and property damage only (PDO). If at least one person died within the first of month of the collision date, it was referred to as fatal collision (Transport Canada, 2018). Injuries refer to those collisions which did not cause death of any person, but which resulted in body injuries for at least one person. All fatal and injury collisions were reported, whereas PDO collisions were reported only if the damage exceeds some specific threshold. 
Sarhan (2004) calculated the number of total collisions on acceleration lanes based on the location of collision, which are referred to as total collisions in further sections. The number of total collisions ranged from zero to eight. Four out of 16 study sites had zero collisions on acceleration lane. Variation of total collisions at $16 \mathrm{SCL}$ sites is shown in Figure 3.9.

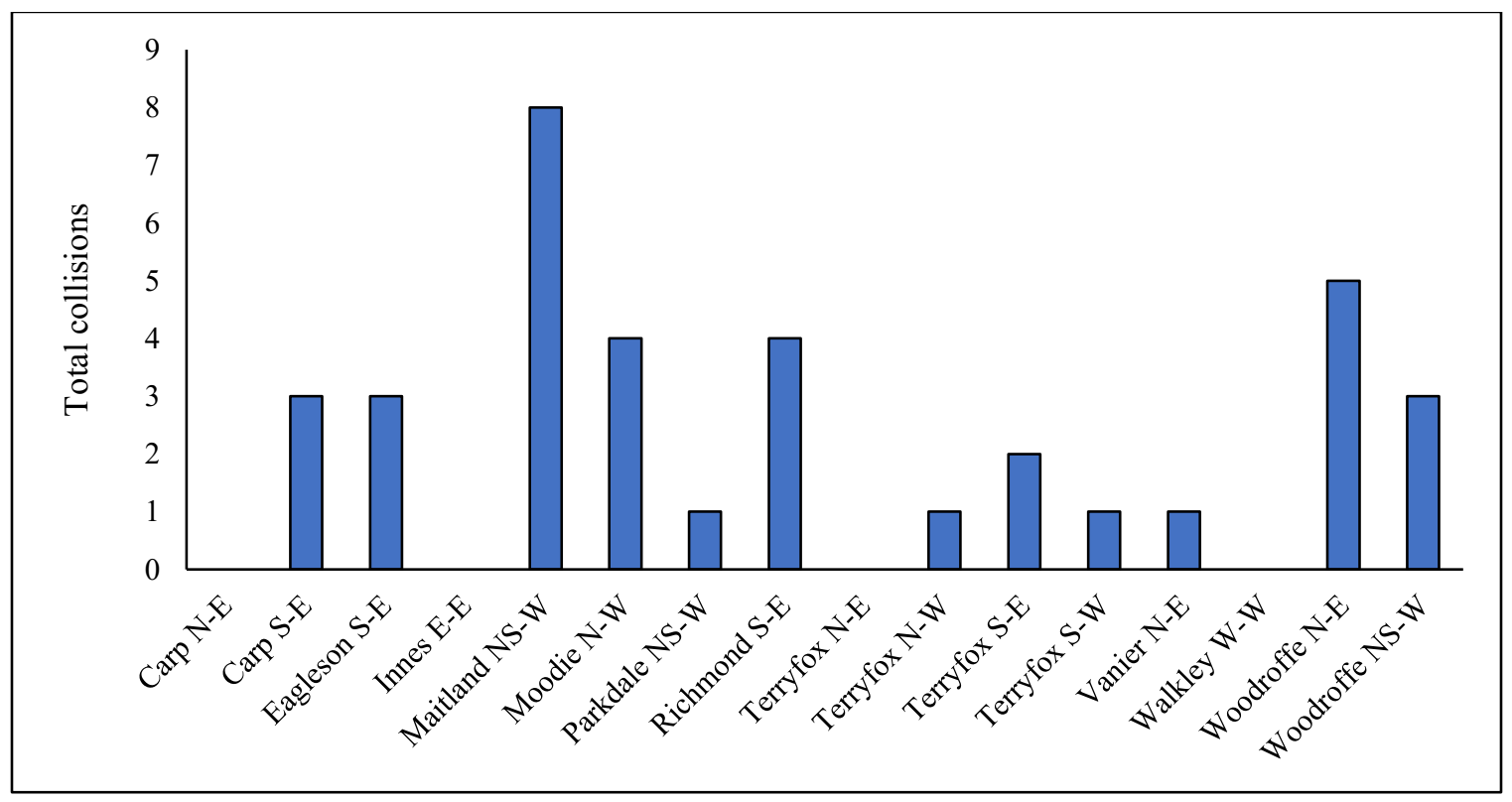

Figure 3.9: Total Collisions on Acceleration Lane during 1998 - 2002.

\subsubsection{Average Annual Daily Traffic}

Average annual daily traffic (AADT) data on freeway through lanes, entrance SCL and exit SCL at the study sites was received from Ahammed (2005). Entrance SCL volume varied from 1,263 veh/day to $14,811 \mathrm{veh} /$ day whereas AADT on the through freeway lanes varied from 11,705 veh/day to $66,600 \mathrm{veh} /$ day. Total AADT which is the sum of AADT at 
entrance SCL, through lanes and exit SCL varied between $16,316 \mathrm{veh}$ /day to 77,526 veh/day. All these numbers are presented in the Figure 3.10.

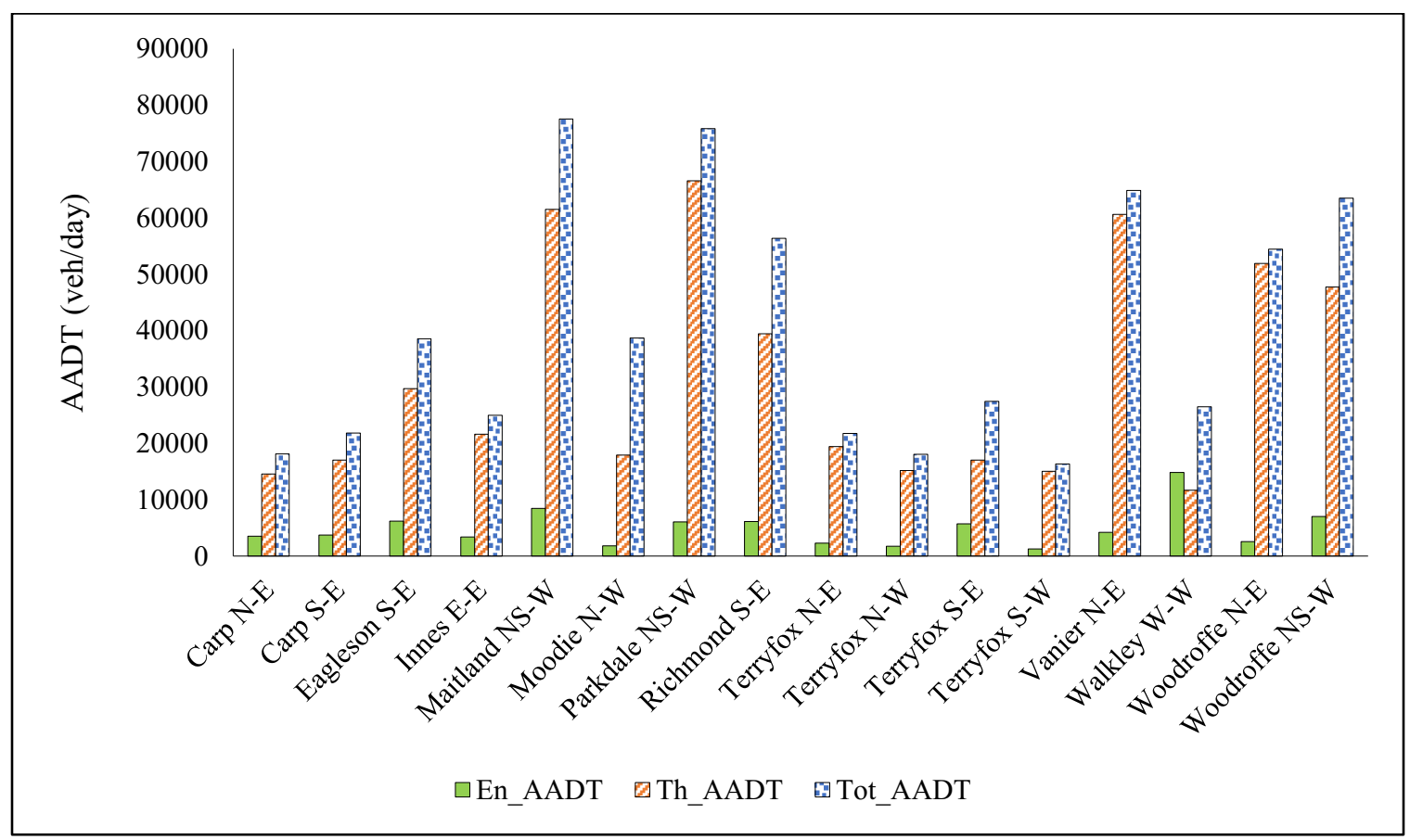

Figure 3.10: Average Annual Daily Traffic at Study Sites.

\subsection{Traffic Volume on F2L and Lane Ratio}

In this study, the ratio of F2L volume to FRL volume is referred to as lane ratio. Since the traffic volume data corresponding to F2L was not available from the past studies, the author of this study had requested Ministry of Transportation (MTO) of Ontario for the traffic volume data at the study sites. Due to lack of traffic monitoring cameras at some of the requested locations, the author received the 1-hour recordings of traffic movements for only twelve of the sixteen study sites. Hourly traffic volumes of FRL and F2L were counted from those videos in order to calculate the lane ratio. The percentage of HV on FRL and 
F2L were also obtained from the videos. As shown in Table 3.8, the traffic volume on F2L ranged from $643 \mathrm{veh} / \mathrm{h}$ to $1633 \mathrm{veh} / \mathrm{h}$. The value of lane ratio ranged from 0.81 to 2.42 and the percentages of $\mathrm{HV}$ on F2L varied from $7.3 \%$ to $12.7 \%$.

Table 3.8: Summary of Data Received from MTO.

\begin{tabular}{|l|c|c|c|c|c|}
\hline \multicolumn{1}{|c|}{ SCL Site } & $\begin{array}{c}\text { FRL } \\
\text { Volume } \\
\text { (veh/h) }\end{array}$ & $\begin{array}{c}\text { Percentage } \\
\text { of HV on } \\
\text { FRL (\%) }\end{array}$ & $\begin{array}{c}\text { F2L } \\
\text { Volume } \\
\text { (veh/h) }\end{array}$ & $\begin{array}{c}\text { Percentage } \\
\text { of HV on } \\
\text { F2L (\%) }\end{array}$ & $\begin{array}{c}\text { Lane Ratio = } \\
\text { F2L volume/ } \\
\text { FRL volume }\end{array}$ \\
\hline Carp N-E & 374 & 16.6 & 643 & 11.4 & 1.72 \\
\hline Eagleson S-E & 860 & 10.9 & 1397 & 10.7 & 1.62 \\
\hline Innes E-E & 916 & 19.1 & 741 & 7.3 & 0.81 \\
\hline Maitland NS-W & 1258 & 10.5 & 1633 & 10.8 & 1.30 \\
\hline Moodie N-W & 643 & 15.2 & 1255 & 11.2 & 1.95 \\
\hline Parkdale NS-W & 1320 & 9.7 & 1576 & 9.8 & 1.19 \\
\hline Terryfox N-E & 324 & 27.5 & 783 & 12.4 & 2.42 \\
\hline Terryfox S-E & 465 & 13.5 & 823 & 11.1 & 1.77 \\
\hline Vanier N-E & 1112 & 22.2 & 1284 & 12.7 & 1.15 \\
\hline Walkley W-W & 751 & 17.4 & 755 & 10.1 & 1.01 \\
\hline Woodroffe N-E & 956 & 11.5 & 1170 & 13.1 & 1.22 \\
\hline Woodroffe NS-W & 1061 & 10.9 & 1552 & 10.3 & 1.46 \\
\hline
\end{tabular}

Hourly traffic volumes on FRL and F2L and percentage of $\mathrm{HV}$ on FRL and F2L are displayed in Figure 3.11 and Figure 3.12, whereas Figure 3.13 shows the variation of lane ratio among various SCL sites. 


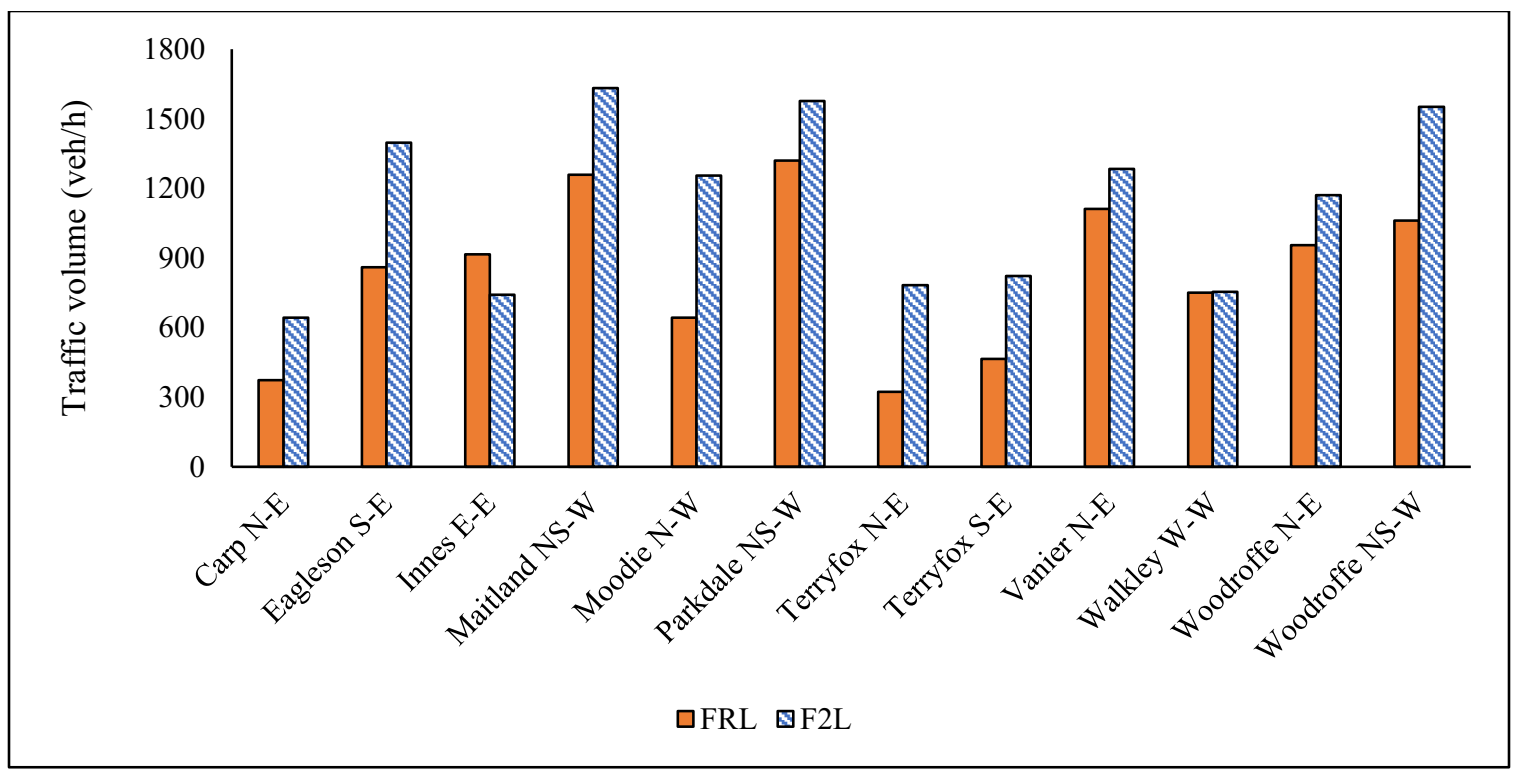

Figure 3.11: Traffic Volume on FRL and F2L.

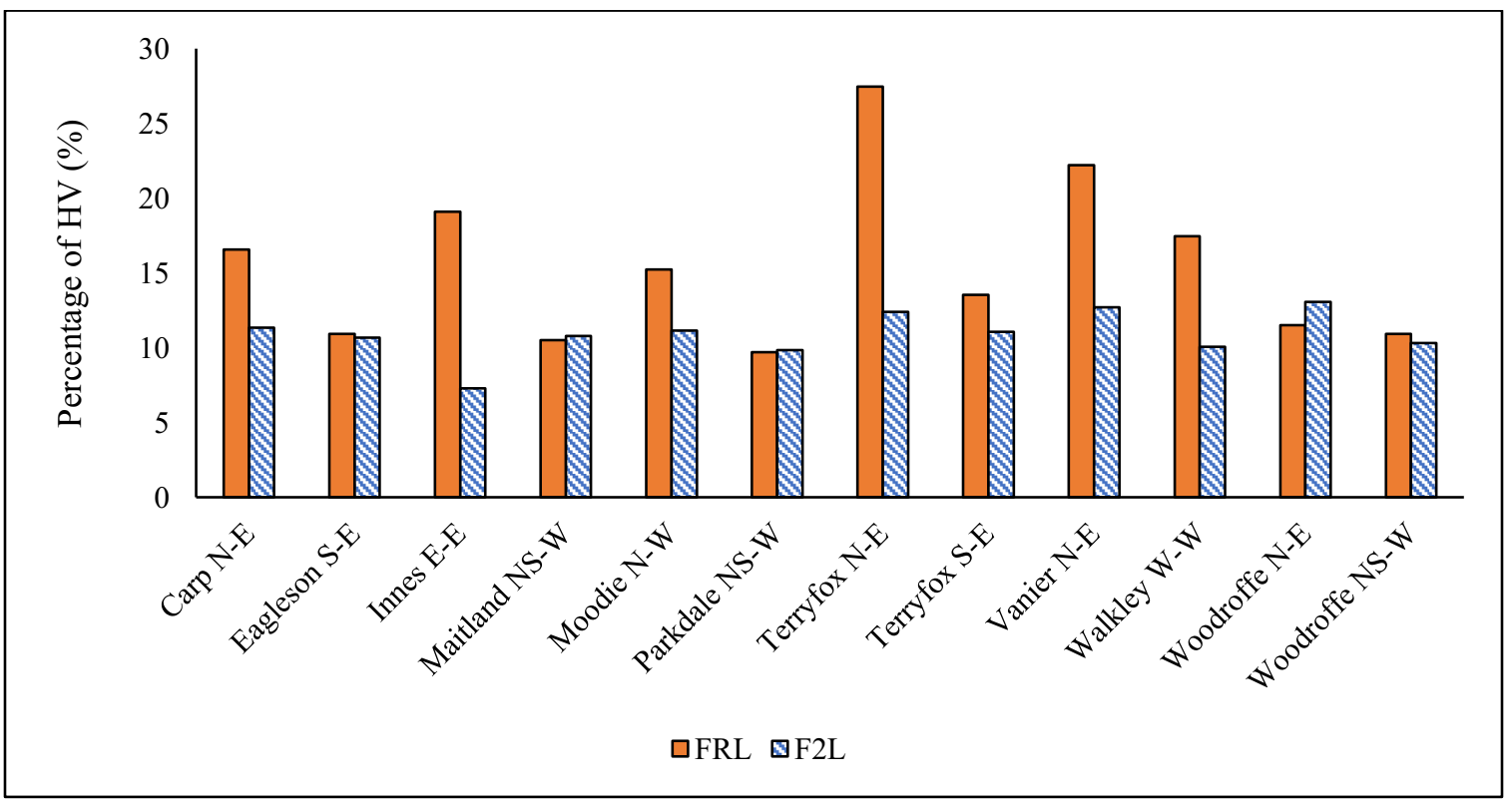

Figure 3.12: Percentage of HV on FRL and F2L. 


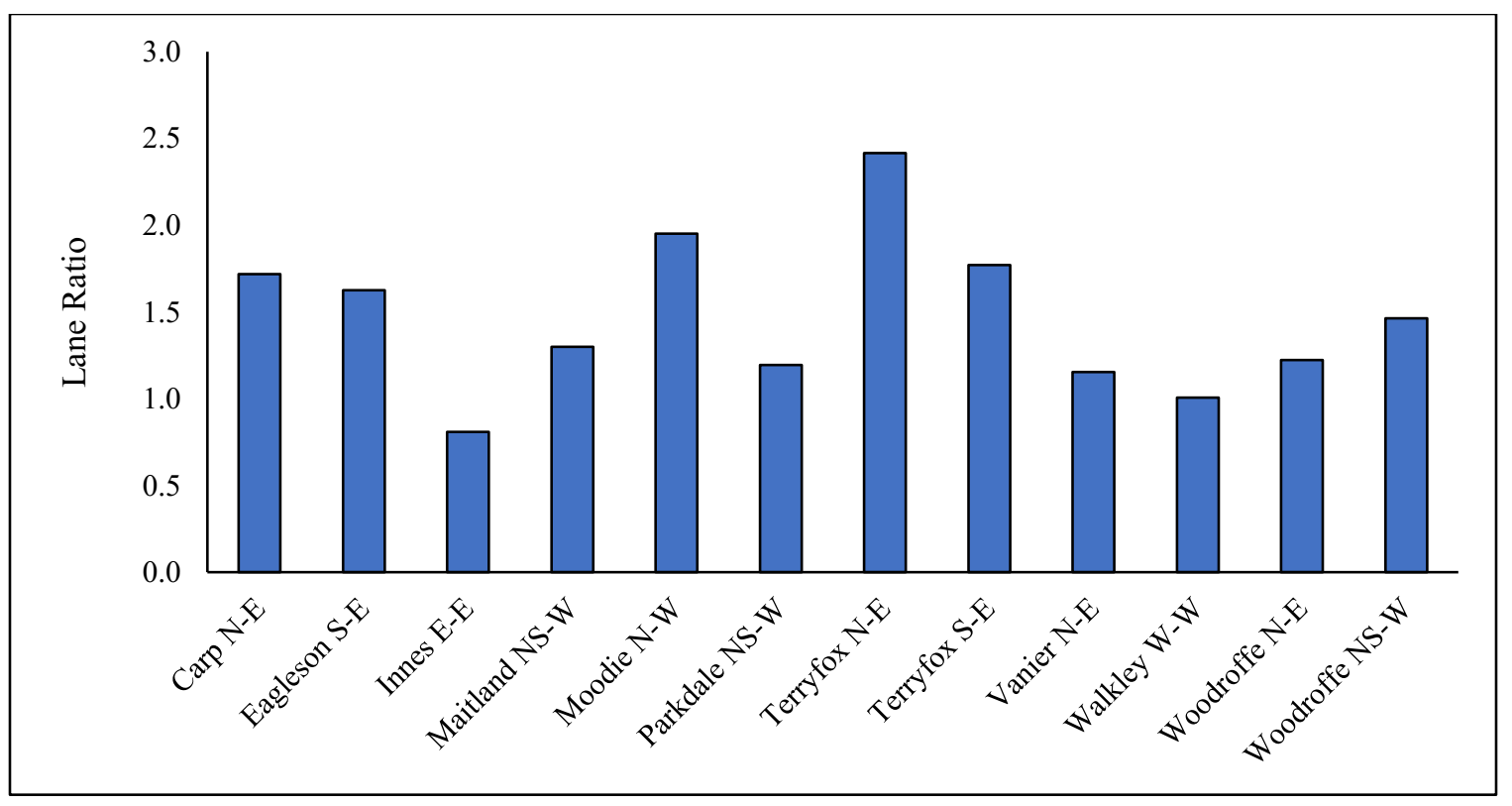

Figure 3.13: Lane Ratio at Various SCL Sites.

\subsection{Normality Tests for Parameters}

As an initial step before developing the simulation models, normality tests such as Kolmogorov-Smirnov (K-S) test and Shapiro-Wilk (S-W) were conducted on different parameters of study sites by Fatema (2012). Statistical distribution of each parameter was verified by using the available data at all study sites. It was found that the parameters gore speed, merge speed, FRL vehicle speed and the acceleration rate follow a normal distribution at $5 \%$ level of significance. Other parameters, lane ratio and percentage of $\mathrm{HV}$ on F2L were not examined for normality in the previous studies. Hence, normality tests for these two parameters were conducted in the present study. 


\subsubsection{Normality Test for Lane Ratio}

SPSS software was used to test the normality of lane ratio at $5 \%$ level of significance using K-S and S-W tests and the results are given below:

Null Hypothesis $\left(\mathrm{H}_{0}\right)$ : Sample data follows a normal distribution.

Alternate Hypothesis $\left(\mathrm{H}_{\mathrm{a}}\right)$ : Sample data does not follow a normal distribution.

The test statistics of K-S test and S-W test were, $0.148(p$-value $=0.200)$ and $0.965(p$ value $=0.853$ ) respectively.

In both the tests, $p$-value is greater than $5 \%$. Hence, the null hypothesis could not be rejected which indicates that the sample data for the lane ratio follows a normal distribution.

\subsubsection{Normality Tests for Percentage of HV on F2L}

Normality of percentage of $\mathrm{HV}$ on F2L was tested at 5\% level of significance using K-S and S-W tests and the results are mentioned below:

Null Hypothesis $\left(\mathrm{H}_{0}\right)$ : Sample data follows a normal distribution

Alternate Hypothesis $\left(\mathrm{H}_{\mathrm{a}}\right)$ : Sample data does not follow a normal distribution

The test statistics of K-S test and S-W test were, $0.169(p$-value $=0.200)$ and $0.925(p$ value $=0.332$ ) respectively.

In both the tests, $p$-value is greater than $5 \%$. Hence, the null hypothesis could not be rejected, and the sample data for percentage of HV on F2L follows a normal distribution. 


\section{Chapter 4: REGRESSION ANALYSIS OF SPEED PARAMETERS}

Input parameters required for simulation have been listed and explained in Chapter 3. It was also mentioned that the complete field data were not available for all the 16 SCL sites and is only available for the full-profile sites. This chapter provides the relation between various parameters and equations that were used to determine the gore speed, merge speed and acceleration rate of simulated vehicles. Regression analysis was carried out to relate the input parameters and the missing data for merge-speed-only sites were calculated using the developed regression models. It was assumed that the design speed of ramp controlling curve is equal to the $85^{\text {th }}$ percentile gore speed. These modelling attempts were done to predict the input parameters such as merge speed and acceleration rates of vehicles using the gore speed. Since the gore speed data were not available at all the SCL sites, the available field data of merge speed were used to develop a regression model for calculating the $85^{\text {th }}$ percentile gore speed. After determining the $85^{\text {th }}$ percentile gore speed, a regression model was developed to predict the gore speed of the SCL vehicles. And finally, regression models were developed to predict merge speed and acceleration rates of SCL vehicles using the calculated gore speed.

\subsection{Model for $85^{\text {th }}$ Percentile Gore Speed}

Since the merge speed data was available at all study SCL sites, Fatema et al. (2014) developed a regression model relating $85^{\text {th }}$ percentile gore speed to the merge speed as following:

$$
\text { gore85thSpeed }=-10.627+1.414 * \text { MergeSpeed }\left(R^{2}=0.74\right)
$$

where, 
gore 85 thSpeed $=85^{\text {th }}$ percentile gore speed $(\mathrm{m} / \mathrm{s})$

MergeSpeed $=$ merge speed at the study site $(\mathrm{m} / \mathrm{s})$

\subsection{Model for Mean Gore Speed}

Gore speed data were available only for full-profile sites, and for merge-speed-only sites mean gore speed needed to be calculated from the available data. Fatema et al. (2014) developed a model relating the mean gore speed to the $85^{\text {th }}$ percentile gore speed. Regression analysis was performed using the existing field data and the following relationship was found:

meangoreSpeed $=-0.287+0.922 *$ gore 85 thSpeed $\left(R^{2}=0.99\right)$

where,

meangoreSpeed $=$ gore speed at the study site $(\mathrm{m} / \mathrm{s})$

gore85thSpeed $=85^{\text {th }}$ percentile of the gore speed $(\mathrm{m} / \mathrm{s})$

Another equation for standard deviation of the gore speed was developed using the regression analysis as following:

sdGoreSpeed $=0.446+0.069 *$ gore 85 thSpeed $\left(R^{2}=0.65\right)$

where,

sdGoreSpeed $=$ standard deviation of gore speed $(\mathrm{m} / \mathrm{s})$

gore 85 thSpeed $=85^{\text {th }}$ percentile of gore speed $(\mathrm{m} / \mathrm{s})$ 
While the Equations 4.1, 4.2, 4.3 were adopted from Fatema et al. (2014), Equations 4.4 and 4.5 were developed by the author of this study using individual vehicle data received from Ahammed (2005).

\subsection{Model for Merge Speed}

After having calculated the gore speed for all the sites, an attempted was made to relate the merge speed of each vehicle to its gore speed. The type of vehicle whether it is a PC or a $\mathrm{HV}$ was also included as an independent variable in the regression analysis and was found to be significant at 5\% level of significance. For the variable vehType, a value of ' 1 ' was assigned for a HV and ' 0 ' for a PC. The equation to predict the merge speed considering gore speed and vehicle type as independent variables is given below:

$$
\begin{aligned}
& \text { MergeSpeed }=17.42+0.014 *\left(\text { goreSpeed }^{2}\right)-0.95 * \text { vehType } \\
& \left(R^{2}=0.74\right),(S E E=1.842)
\end{aligned}
$$

where,

MergeSpeed $=$ merge speed of SCL vehicle $(\mathrm{m} / \mathrm{s})$

goreSpeed $=$ gore speed of SCL vehicle $(\mathrm{m} / \mathrm{s})$

vehType $=$ type of vehicle

A SCL vehicle with a specific gore speed will have a merge speed value, which follows a normal distribution with the mean as calculated from Equation 4.4 and SD equal to the SEE of the model. 


\subsection{Model for Acceleration Rate}

In a similar way to the merge speed, regression analysis was performed to relate the acceleration rate of each SCL vehicle to its gore speed and the vehicle type as shown in equation 4.5. For the variable vehType, a value of ' 1 ' was assigned for a $\mathrm{HV}$ and ' 0 ' for a PC.

$$
\text { AccRate }=1.53-0.05 * \text { goreSpeed }-0.19 * \text { vehType }
$$

$\left(R^{2}=0.35\right),(S E E=0.288)$

where,

AccRate $=$ acceleration rate of each SCL vehicle $\left(\mathrm{m} / \mathrm{s}^{2}\right)$

goreSpeed $=$ gore speed each SCL vehicle $(\mathrm{m} / \mathrm{s})$

vehType $=$ type of vehicle

A SCL vehicle with a specific gore speed will have an acceleration rate value, which follows a normal distribution with the mean as calculated from Equation 4.5 and SD equal to the SEE of the model.

Using the models mentioned above, missing input parameters for the merge-speed-only SCL sites were calculated. Input parameters considered for simulating the vehicles are shown in Table 4.1. 
Table 4.1: Input Parameters for Study Sites.

\begin{tabular}{|c|c|c|c|c|c|c|c|}
\hline \multirow{2}{*}{ Study site } & \multirow{2}{*}{$\begin{array}{c}\text { SCL } \\
\text { length } \\
(\mathbf{m})\end{array}$} & \multirow{2}{*}{$\begin{array}{c}\text { 85th } \\
\text { percentile } \\
\text { Gore speed } \\
(\mathbf{k m} / \mathbf{h})\end{array}$} & \multicolumn{2}{|c|}{$\begin{array}{l}\text { Freeway speed } \\
\qquad(\mathrm{km} / \mathrm{h})\end{array}$} & \multirow{2}{*}{$\begin{array}{c}\text { FRL } \\
\text { volume } \\
\text { (veh/h) }\end{array}$} & \multirow{2}{*}{$\begin{array}{l}\text { Percentage } \\
\text { of HV on } \\
\text { FRL (\%) }\end{array}$} & \multirow{2}{*}{$\begin{array}{c}\text { SCL } \\
\text { volume } \\
\text { (veh/h) }\end{array}$} \\
\hline & & & mean & $\begin{array}{l}\text { standard } \\
\text { deviation }\end{array}$ & & & \\
\hline Carp N-E & 425 & 90.72 & 109.73 & 8.17 & 521 & 7.4 & 193 \\
\hline Carp S-E & 430 & 99.85 & 109.29 & 7.52 & 709 & 16.3 & 271 \\
\hline $\begin{array}{l}\text { Eagleson } \\
\text { S-E }\end{array}$ & 327 & 95.71 & 98.29 & 7.90 & 1257 & 11.0 & 429 \\
\hline Innes E-E & 354 & 90.16 & 106.31 & 7.29 & 848 & 18.8 & 237 \\
\hline $\begin{array}{l}\text { Maitland } \\
\text { NS-W }\end{array}$ & 468 & 62.51 & 96.64 & 8.65 & 1229 & 11.1 & 594 \\
\hline $\begin{array}{l}\text { Moodie } \\
\text { N-W }\end{array}$ & 323 & 91.77 & 97.80 & 7.85 & 1244 & 11.8 & 111 \\
\hline $\begin{array}{l}\text { Parkdale } \\
\text { NS-W }\end{array}$ & 188 & 69.73 & 98.49 & 11.75 & 588 & 5.1 & 422 \\
\hline $\begin{array}{l}\text { Richmond } \\
\text { S-E }\end{array}$ & 321 & 83.28 & 98.99 & 10.30 & 492 & 6.1 & 437 \\
\hline $\begin{array}{l}\text { Terryfox } \\
\text { N-E }\end{array}$ & 418 & 88.83 & 103.43 & 8.18 & 685 & 18.7 & 188 \\
\hline $\begin{array}{l}\text { Terryfox } \\
\text { N-W }\end{array}$ & 419 & 100.05 & 108.27 & 7.51 & 661 & 20.2 & 161 \\
\hline $\begin{array}{l}\text { Terryfox } \\
\text { S-E }\end{array}$ & 437 & 106.78 & 104.21 & 8.91 & 785 & 15.0 & 351 \\
\hline $\begin{array}{l}\text { Terryfox } \\
\text { S-W }\end{array}$ & 395 & 92.56 & 107.61 & 8.40 & 704 & 20.0 & 95 \\
\hline Vanier N-E & 363 & 83.61 & 90.41 & 8.11 & 1195 & 13.4 & 294 \\
\hline $\begin{array}{l}\text { Walkley } \\
\text { W-W }\end{array}$ & 422 & 80.28 & 106.14 & 10.84 & 345 & 13.6 & 965 \\
\hline $\begin{array}{l}\text { Woodroffe } \\
\text { N-E }\end{array}$ & 248 & 85.38 & 96.13 & 8.62 & 966 & 11.0 & 191 \\
\hline $\begin{array}{l}\text { Woodroffe } \\
\text { NS-W }\end{array}$ & 346 & 78.85 & 102.87 & 10.42 & 886 & 11.5 & 354 \\
\hline
\end{tabular}




\section{Chapter 5: METHODOLOGY}

In this study, various merging maneuvers are examined to study the variations in probability of forced merging of SCL drivers in the regular vehicles and CV environment. Vehicles without the connectivity are referred to as regular vehicles in this study. The simulation logic, methodologies used for modelling the freeway merging process, assumptions considered in the modelling and details of input parameters are explained in this chapter.

\subsection{Introduction}

In this study, microscopic simulation method is used to model the merging process considering the gap searching and acceleration behaviour of SCL drivers. The model output provides the probability of uncomfortable or forced merging based on the gap availability and required acceleration behaviour. The output PNC quantifies the combined probability of forced merging of each SCL vehicle when the available gap is not enough or when the required acceleration rate to reach the merge location is not within the acceptable acceleration limits. The combined PNC is calculated both for vehicles without connectivity and with connectivity and the details are explained in further sections.

\subsubsection{Microscopic Simulation}

In general, microscopic simulation models are well-suited for detailed analysis of individual vehicle interactions. Macroscopic simulation models are more appropriate for analysis of larger networks and mesoscopic models combine the characteristics of both microscopic and macroscopic models. Microscopic simulation models use car-following and lane-changing models to simulate the driver behaviour and vehicle positions in the 
required time steps. These models provide detailed performance measures to examine the capacity, demand or facility operations and the outputs are based on the stochastic behaviour of drivers (Campbell, Alexiadis, \& Krechmer, 2015).

\subsubsection{Monte Carlo Simulation}

As stated by (Singh et al., 2007), Monte Carlo Simulation is a "technique to obtain statistical properties of the output of a system given the properties of inputs and the system." In this method, inputs are transformed by means of a mathematical model of the system which is developed according to the important characteristic of the system. The major steps in this simulation process are gathering the input data, developing a model of the system, performing the simulation using the input data and the model and finally analyzing the output. In Monte Carlo method, random numbers are generated for each input variable following probability distribution of the parameter, and the accuracy of the output depends on the number of experiments performed using the input data (Singh et al., 2007).

\subsection{Modeling the Merging Process}

In the current study, three simulation methods were developed to examine the various merging maneuvers for regular vehicles and CV's. Vehicles without the connectivity are referred to as regular vehicles in this study.

Method 1: In the first modelling technique, driver behaviour in regular vehicles was modelled based on the following steps and will be referred to as Method 1 in further discussions:

Step 1: Each SCL vehicle enters onto the SCL with an initial speed which is the gore speed. 
Step 2: All the available total gaps in FRL and the distances and times required for the SCL vehicle to merge at the available gaps are calculated.

Step 3: PNC due to gap failure is calculated at all the available gaps in FRL.

Step 4: Mean acceleration rates required to reach the merging locations are calculated and PNC due to acceleration failure is calculated.

Step 5: Combined PNC due to total gap failure and acceleration failure is calculated using the PNC values due to gap failure and acceleration failure.

Step 6: Mean combined PNC of all simulated SCL vehicles is the output from this method.

Method 2: This modelling technique is similar to Method 1, but the simulation is developed for vehicles with connectivity and is termed as cooperative merging process. This is based on the below mentioned steps and will be referred to as Method 2 in further sections:

Step 1: Each SCL vehicle enters onto the SCL with an initial speed which is the gore speed.

Step 2: All the available total gaps in FRL are calculated.

Step 3: Targeting the gap to be reached based on the mean merge speed and mean acceleration rate, the mean time to merge and the mean location to merge are calculated.

Step 4: If the targeted total gap is not acceptable by SCL driver, lag vehicle which is behind the FRL vehicle in the simulation limits, is requested to decelerate to create the sufficient gap. 
Step 5: Combined PNC due to gap failure and acceleration failure is calculated in order to merge at the gap created by lag vehicle.

Step 6: Mean combined PNC of all simulated SCL vehicles is the output from this method

Method 3: This modelling technique is similar to Method 2, but the lag vehicle will try to create the required gap in an alternative approach which is termed as lane changing advisory method. This is based on the below mentioned steps and will be referred to as Method 3 in further sections:

Step 1: Each SCL vehicle enters onto the SCL with an initial speed which is the gore speed.

Step 2: All the available total gaps in FRL are calculated.

Step 3: Targeting the gap to be reached based on the mean merge speed and mean acceleration rate, the mean time to merge and the mean location to merge are calculated.

Step 4: If the targeted total gap is not acceptable by SCL driver, lag vehicle which is behind the FRL vehicle in the simulation limits, is requested to make a lane change to the left freeway lane to create the sufficient gap.

Step 5: Combined PNC due to gap failure and acceleration failure is calculated in order to merge at the gap created by lag vehicle.

Step 6: Mean combined PNC of all simulated SCL vehicles is the output from this method.

The flowcharts of modelling the merging process are provided by Figure 5.1 and Figure 5.2 . 


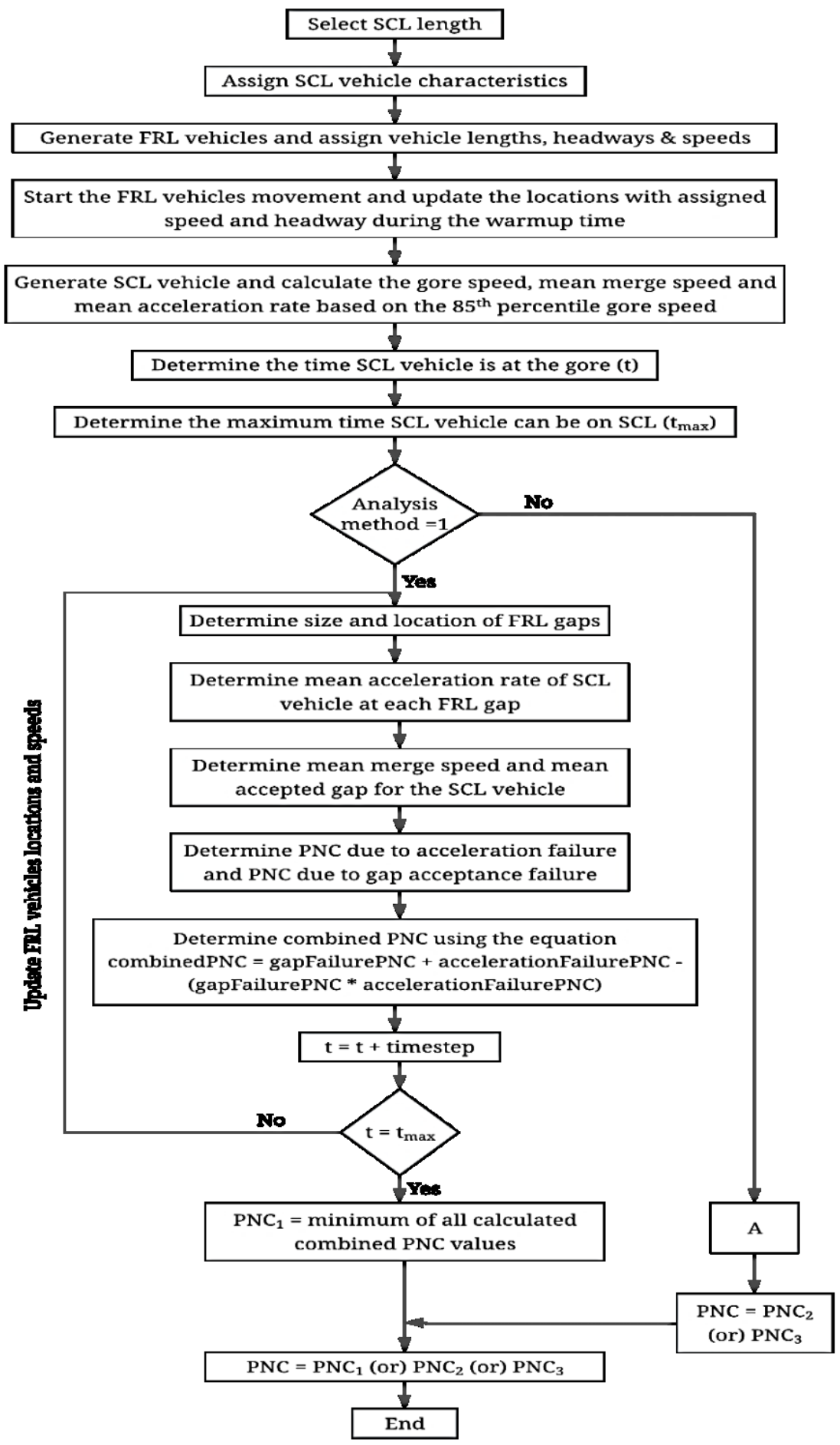

Figure 5.1: Flow Chart of the Overall Simulation Process 


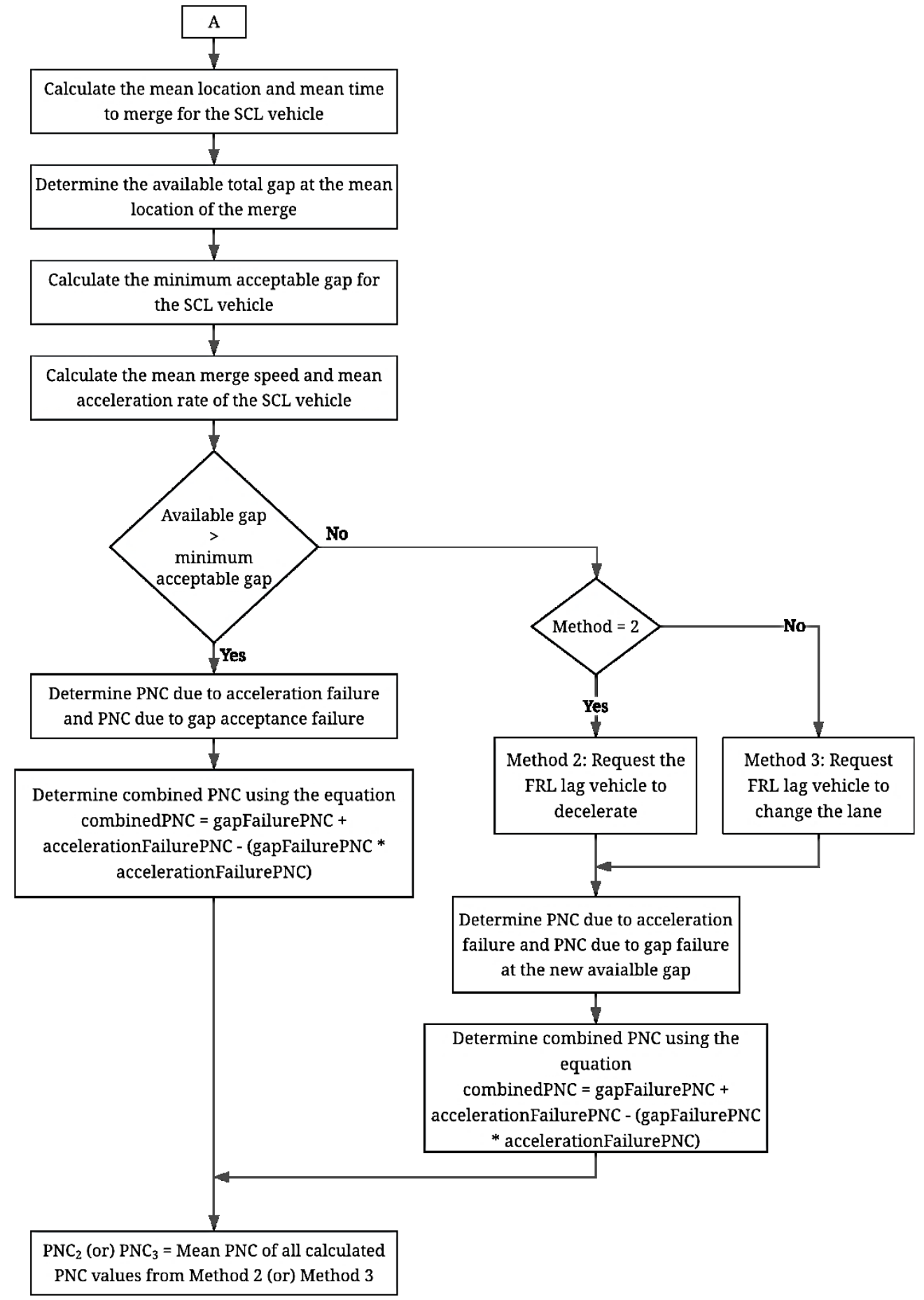

Figure 5.2: Flow Chart A for the Simulation Methods 2 and 3 
To simulate the three merging processes, the input parameters considered are FRL traffic volume, F2L traffic volume, percentages of PC and HV on FRL, speed of FRL vehicles, percentages of $\mathrm{PC}$ and $\mathrm{HV}$ on F2L, speed of F2L vehicles, SCL vehicles' gore speed, merge speed, acceleration rate, traffic volume on SCL and the SCL length.

\subsubsection{Framework of the Simulation Models}

In the three simulation techniques of this study, two lanes were considered on the freeway which are freeway right most lane and freeway second lane and one lane on the SCL. MATLAB R2018a version was used for simulating the merging process. At the beginning of simulation, SCL length was assigned and location of gore, distance considered behind the gore location for positioning the SCL vehicles and the additional distance after end of SCL length were also assigned. Then SCL volume, minimum headway of SCL vehicles, design speed of ramp controlling curve were decided and SCL vehicles were positioned according to their headways. The gore speed of SCL vehicles and vehicle lengths were assigned and the SCL vehicles were positioned according to the headways. Characteristics of freeway lanes such as traffic volume, vehicle speeds and headways were assigned. Simulation was run for five minutes which is considered as warmup time to create the platoon of SCL vehicles and PNC was not calculated during the warmup time. The position of vehicles has been updated for every timestep which is 0.1 second in this study. The simulation process was done for a certain number of runs for each method details of which will be explained in further sections. In each run of the simulation process, SCL vehicles were generated and combined PNC due to gap failure and acceleration failure was calculated for each SCL vehicle according to the steps described for Method 1, Method 2 and Method 3. Failure to find an acceptable total gap in the FRL for comfortable merging 
is referred to as gap failure and if the required acceleration rate to complete the merging is above the acceptable acceleration rates, then it is referred as acceleration failure. The output of the model in each simulation technique is the mean PNC and standard deviation of PNC of all the runs for which the simulation was performed. Detailed explanation of generating SCL vehicles, generating FRL vehicles and calculating PNC for the developed three approaches are provided in the following sections.

\subsubsection{SCL Vehicle Characteristics and Input Parameters}

In the simulation process, driving characteristics were assigned to each SCL vehicle based on the field data using the Monte Carlo Simulation technique. Assumptions made for simulating the SCL vehicles are listed below following the details of input parameters:

- SCL length was measured from the end of ramp controlling curve to the end of acceleration lane. The distance from the gore to the end of ramp controlling curve was assumed as $50 \mathrm{~m}$.

- Zhang et al. (2007) collected headway data on an urban freeway during different times of the day using the advanced loop event data analyzer and observed a minimum headway between 0.375 seconds to 0.453 seconds. In this study, the minimum headway on both SCL and FRL was assumed as 0.5 seconds.

- The design speed of ramp controlling curve was assumed to be equal to the $85^{\text {th }}$ percentile gore speed.

- The simulation of each SCL vehicle begins at the end of ramp controlling curve and ends at the end of SCL length. 
- Each SCL vehicle accelerates until it reaches the merge speed from the gore speed and then travels with a constant speed while searching for a suitable gap.

- All vehicles on the SCL were assumed to be passenger cars as the percentages of heavy vehicles on SCL were relatively low when compared with the percentages on FRL.

- Kim et al. (2008) conducted a study on the gap acceptance behaviour of SCL drivers during the merging process on to a freeway. It was observed that the drivers do not interact with the FRL traffic when the available gap is more than 6 seconds. Therefore, based on the literature it was assumed that maximum required gap which can be accepted by all the SCL drivers is 6 seconds in this study. If the available gap is greater than 6 seconds, then that vehicle was considered to be merged comfortably and the PNC was assigned as zero.

\subsection{2.a Vehicle Lengths}

The lengths of vehicles on SCL and FRL were required for converting the headways to gaps between the vehicles. Headway is measured from the front of the lead vehicle to the front of the lag vehicle whereas gap is measured from the rear of the lead vehicle to the front of the lag vehicle. Fatema, (2012) examined the distribution of different PC lengths such as small size, mid-size, luxury, sports and SUV from the General Motors cars catalogue. It was observed that the PC lengths follow a uniform distribution and vary between $4.399 \mathrm{~m}$ to $5.207 \mathrm{~m}$. According to US Census Bureau (2004), 98 percentile length of the trucks was found to be $12.5 \mathrm{~m}$. Since the percentage of HV on FRL was very less when compared to the percentage of $\mathrm{PC}$, a constant length of $12.5 \mathrm{~m}$ was assigned to all 
$\mathrm{HV}$ in the simulation. If a vehicle is a passenger car, length was assigned randomly from the uniform distribution of PC lengths.

\subsection{2.b Gore Speed of SCL Vehicles}

The mean gore speed and standard deviation of gore speed were calculated using the Equations 4.2 and 4.3. As mentioned in the Section 3.4, gore speed of SCL vehicles follows a normal distribution. Random gore speeds were generated following a normal distribution and were assigned to the SCL vehicles based on the traffic volume on SCL. Maximum gore speed and minimum gore speed were calculated to fall within the limits of $95 \%$ confidence interval using the mean and standard deviation of gore speed. Any gore speed value that is greater than the maximum limit and less than the minimum limit or less than zero was

eliminated. If the gore speed of lag vehicle is greater than gore speed of lead vehicle and if the headway between the lag and lead vehicles is equal to the minimum headway, then the gore speed of the lag vehicle was adjusted to be equal to the gore speed of the lead vehicle.

\subsection{2.c Headway of SCL Vehicles}

Observing the general trend of vehicle movements, the probability of arriving of any number of vehicles within a specified time period is assumed to follow a Poisson's distribution. For a specific traffic volume, set of probabilities in the range $[0,1]$ can be converted to time headways using the following equation (Garber \& Hoel, 2009) and (Fatema, 2012).

$$
h=-\frac{1}{\lambda} \ln \left(R_{N}\right)
$$

Where 
$h=$ headway $(\mathrm{s})$

$\lambda=$ hourly traffic volume $(\mathrm{veh} / \mathrm{h})$

$R_{N}=$ set of random numbers in the range [0 1]

Headway for the first SCL vehicle in the simulation was set as warmup time and the headways for other SCL vehicles in the simulation were assigned randomly which were calculated using the traffic volume on SCL.

\subsection{2.d Merge Speed of SCL Vehicles}

Using the gore speed, mean merge speed value was calculated from Equation 4.4 and assigned to each SCL vehicle in the simulation. To avoid negative acceleration rates, it was made sure that no SCL vehicle has its mean merge speed less than its gore speed.

\subsection{2.e Acceleration Rate of SCL Vehicles}

In a similar way to the merge speed calculation, the mean acceleration rate was calculated using Equation 4.5 and assigned to SCL vehicle in the simulation. From the regression analysis mentioned in the Section 4.4, standard deviation of acceleration rate was obtained as $0.288 \mathrm{~m} / \mathrm{s}^{2}$.

\subsubsection{Freeway Vehicle Characteristics and Input Parameters}

The available field data about freeway vehicles has been already explained in Chapter 3:. Few assumptions made for simulating the freeway vehicles are listed below following the details of input parameters: 
- An additional distance of $500 \mathrm{~m}$ was considered after the end of SCL to capture the effect of FRL vehicles on the vehicles in SCL area. This was considered as the end point after which FRL vehicle exits the simulation.

- In a study on deceleration behaviour of different types of vehicles, Maurya \& Bokare (2012) noticed that the maximum deceleration rate of different type of vehicles varies from $0.719 \mathrm{~m} / \mathrm{s}^{2}$ to $1.925 \mathrm{~m} / \mathrm{s}^{2}$. Based on the literature and own judgment, the maximum deceleration of freeway vehicles was assumed as $2.0 \mathrm{~m} / \mathrm{s}^{2}$ in this study. It was also assumed that the deceleration rates of all FRL vehicles fall within the $\pm 10 \%$ of maximum deceleration rate. This deceleration rate was used when the FRL lag vehicle requested to slow down to create a sufficient gap for SCL vehicle in Method 2.

\subsection{3.a Headway and Speed Adjustments of FRL Vehicles}

FRL vehicles headways were calculated using Equation 5.1 as explained in Section 5.2.2.c. In order to create the realistic gap patterns between the FRL vehicles, the speeds and headways of lead and lag vehicles were adjusted based on parameters such as minimum headway in FRL, critical headway and deceleration rate of lag vehicle. Headway and speed adjustments were done based on the following conditions:

- No FRL vehicle can have a headway less than the minimum FRL headway.

- A vehicle with minimum headway cannot have a speed higher than its lead vehicle. In this case, the speed of lag vehicle was made equal to the speed of its lead vehicle.

- A lag vehicle with higher speed than its lead vehicle, is required to slow down until it reaches the minimum headway and its speed is equal to lead vehicle's speed. 
- If the initial headway of a lag vehicle is close to minimum headway, the speed of the lag vehicle was reduced to maintain the headway equal to minimum headway and speed equal to lead vehicle's speed.

- In the case of a lag vehicle having higher speed than its lead vehicle, then it will have to start slowing down once it reaches the critical headway.

The equations used to make the above-mentioned adjustments for headways and speeds of FRL vehicles are as following:

\section{Minimum Headway on FRL}

As mentioned in the Section 5.2.2, the minimum headway on FRL considered in this study as per the literature is 0.5 seconds. Minimum headway was also calculated for FRL vehicles in simulation based on the vehicle length, speed of the vehicle and minimum headway as per literature using Equation 2.2.

$$
h_{\text {min }}=\max \left(\left[h_{\text {minFRL }},\left(\frac{\text { vehLengt }_{\text {lead }}}{\text { speed }_{\text {lead }}}+0.5 * h_{\text {minFRL }}\right)\right]\right)
$$

Where

$h_{\min }=$ minimum headway of lag vehicle (s)

$h_{\text {minFRL }}=$ minimum headway on FRL (s)

vehLength $h_{\text {lead }}=$ vehicle length of the lead vehicle $(\mathrm{m})$

speed lead $=$ speed of the lead vehicle $(\mathrm{m} / \mathrm{s})$ 
The minimum headway considered for further calculations was the maximum of minimum headway from the literature and the minimum headway calculated as per characteristics of lead vehicle.

\section{Critical Headway for Lag Vehicle:}

The lag vehicle moving with a higher speed than its lead vehicle has to start slowing down when it reaches the critical headway calculated using Equation 5.3. This equation was derived based on the equations of motion.

$$
h_{\text {crit }}=\frac{\left(\text { speed }_{\text {lag }}-\text { speed }_{\text {lead }}\right)^{2}}{2 * \text { decRate }_{\text {lag }} \text { speed }_{\text {lag }}}+\frac{h_{\mathrm{min} * \text { speed }_{\text {lead }}}}{\text { speed }_{\text {lag }}}
$$

Where

$h_{\text {crit }}=$ critical headway (s)

speed lag $=$ speed of the lag vehicle $(\mathrm{m} / \mathrm{s})$

speed $_{\text {lead }}=$ speed of the lead vehicle $(\mathrm{m} / \mathrm{s})$

$h_{\min }=$ minimum headway of lag vehicle (s)

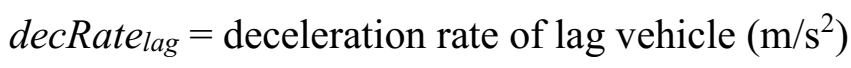

\section{Required Deceleration Rate of Lag Vehicle :}

If lag vehicle is faster than lead vehicle but it may not have to slow down at the beginning of simulation, then the deceleration rate required for the lag vehicle to maintain the minimum headway is as given by Equation 5.4. 


$$
\text { decRate }_{\text {lag }}=\frac{\left(\text { speed }_{\text {lag }}-\text { speed }_{\text {lead }}\right)^{2}}{2 * \operatorname{speed}_{\text {lag }} * h_{\text {lag }}-\operatorname{speed}_{\text {lead }} * h_{\text {min }}}
$$

Where

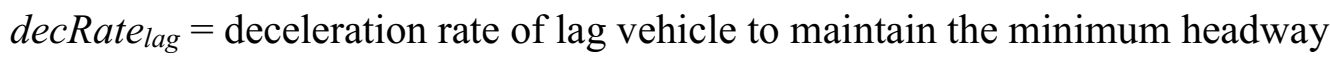

speed $_{\text {lag }}=$ speed of the lag vehicle $(\mathrm{m} / \mathrm{s})$

speed $_{\text {lead }}=$ speed of the lead vehicle $(\mathrm{m} / \mathrm{s})$

$h_{l a g}=$ headway of lag vehicle (s)

$h_{\min }=$ minimum headway of the lag vehicle (s)

If the deceleration rate calculated from Equation 5.4 is higher than the maximum deceleration rate, it indicates that the speed of the lag vehicle is too high at the beginning of simulation. Hence, the speed of the lag vehicle has to be adjusted at the start of the simulation using Equation 5.5:

$$
\text { Adjspeed }_{\text {lag }}=\frac{-b+\sqrt{b^{2}-4 a c}}{2 a}
$$

Substituting,

$$
\begin{aligned}
& a=1 \\
& b=-2 *\left(V_{\text {lead }}+h_{\text {lag }} * \text { decRate }_{\text {lag }}\right) \\
& c=\operatorname{speed}_{\text {lead }} *\left(V_{\text {lead }}+2 * h_{\text {min }} * \text { decRate }_{\text {lag }}\right)
\end{aligned}
$$

where

Adjspeed lag $=$ adjusted speed of the lag vehicle at the beginning of simulation $(\mathrm{m} / \mathrm{s})$ 
speed $_{\text {lead }}=$ speed of the lead vehicle $(\mathrm{m} / \mathrm{s})$

$h_{\text {lag }}=$ headway of lag vehicle (s)

$h_{\min }=$ minimum headway of the lag vehicle (s)

\subsection{3.b Updating Speed and Location of FRL Vehicles}

Speeds, locations and headways for FRL vehicles in the simulation were updated for timestep i.e., $0.1 \mathrm{~s}$. For the first vehicle the simulation, only speed and location were updated because there is no lead vehicle and hence there is no headway for the first vehicle. For all other vehicles, speed, location and headway were updated.

(i) If the speed of lag vehicle is less than speed of the lead vehicle and the headway is increasing, then the vehicle location and headways are updated using Equations 5.6 and 5.7 respectively:

Vehicle Location:

vehLoc $_{i}=$ vehLoc $_{i-1}+$ speed $*$ timestep

Where

$\operatorname{vehLoc}_{i}=$ updated vehicle location $(\mathrm{m})$

$v e h L o c_{i-1}=$ vehicle location before the update $(\mathrm{m})$

speed $=$ speed of the vehicle $(\mathrm{m} / \mathrm{s})$

timestep $=$ time step $(\mathrm{s})$

\section{Headway:}

$$
h_{\text {lag }}=\frac{\text { eehLoc }_{\text {lead }}-\text { vehLoc }_{\text {lag }}}{\text { speed }_{\text {lag }}}
$$


Where

$h_{\text {lag }}=$ headway of the lag vehicle $(\mathrm{s})$

vehLoclead $=$ location of the lead vehicle $(\mathrm{m})$

vehLoclag $=$ location of the lag vehicle $(\mathrm{m})$

speed $_{\text {lag }}=$ speed of the lag vehicle $(\mathrm{m} / \mathrm{s})$

(ii) If the lag vehicle is slower than its lead vehicle but the headway is greater than the critical headway, then the location and headway are updated using the equations 5.6 and 5.7. Along with the location and headway, the minimum headway for the lag vehicle also required to be updated to ensure that it is lower than the critical headway. Minimum headway is updated using Equation 5.8.

$h_{\text {min }}=\max \left(\left[h_{\text {minFRL }},\left(\frac{\text { vehLen }_{\text {lead }}}{\text { speed }_{\text {lead }}}+0.5 * h_{\text {minFRL }}\right)\right]\right)$

Where

$h_{\text {min }}=$ updated minimum headway for the lag vehicle (s)

$h_{\min F R L}=$ minimum headway on FRL (s)

$v^{\text {vehLen lead }}=$ length of the lead vehicle $(\mathrm{m})$

speed lead $=$ speed of the lead vehicle $(\mathrm{m})$

If the headway updated using Equation 5.7 is less than the critical headway of the lag vehicle, the headway is adjusted to be equal to the critical headway of the lag vehicle. 
(iii) If the lag vehicle is slower than its lead vehicle and the headway is smaller than the critical headway, then the lag vehicle has to slow down to maintain its headway greater than the critical headway. The headway and minimum headway of the lag vehicle are updated using Equations 5.7 and 5.8 respectively. The location and speed of the lag vehicle are updated using Equations 5.9 and 5.10 respectively.

\section{Location:}

$$
v_{e h L o c}=v_{\text {ehLoc }}+1+\left(\text { speed }^{*} \text { timestep }\right)-\left(0.5 * \text { decRate }^{*} \text { timestep }^{2}\right)
$$

Where

vehLoc $_{i}=$ updated vehicle location $(\mathrm{m})$

$\operatorname{vehLoc}_{i-1}=$ vehicle location before the update $(\mathrm{m})$

speed $=$ speed of the vehicle $(\mathrm{m} / \mathrm{s})$

timestep $=$ time step in this study $(\mathrm{s})$

decRate $=$ deceleration arete of the vehicle $\left(\mathrm{m} / \mathrm{s}^{2}\right)$

\section{Speed:}

$$
\text { speed }_{i}=\text { speed }_{i-1}-\left(\text { decRate }^{*} \text { timestep }\right)
$$

Where

speed $_{i}=$ updated speed of the vehicle $(\mathrm{m} / \mathrm{s})$

speed $_{i-1}=$ speed of the vehicle before updating $(\mathrm{m} / \mathrm{s})$

timestep $=$ time step in this study $(\mathrm{s})$

decRate $=$ deceleration arete of the vehicle $\left(\mathrm{m} / \mathrm{s}^{2}\right)$ 
If the updated headway of the lag vehicle is lower than the minimum headway of the lag vehicle, then the headway is made equal to the minimum headway of the lag vehicle.

\subsection{Evaluation of PNC}

After assigning the characteristics of SCL vehicles and FRL vehicles, the simulation of FRL vehicles will begin. After running the simulation of FRL vehicles until the warmup time, each SCL vehicle will be released and the combined PNC due to gap failure and acceleration failure will be evaluated using the three simulation techniques developed.

\subsubsection{Calculation of Available Gaps in Freeway Lanes}

In FRL, total gaps were calculated from the gore location to the end point of the SCL. In F2L, gaps were recorded from the beginning of the simulation area which includes the additional distance behind the gore to the end of SCL. Different scenarios considered for effective calculation of available gaps are

(i) There are no FRL vehicles in the SCL area or both the last vehicle downstream the SCL and first vehicle upstream the SCL are not within the simulation limits. This might happen when the FRL traffic volume is very low.

(ii) The last vehicle downstream SCL is beyond the simulation limits. Merging location for the last available gap required to be adjusted if the lag vehicle is upstream the gore.

(iii) The first vehicle upstream the SCL vehicle is not within the simulation limits. This vehicle needs to be removed and first and last gaps need to be adjusted. 
In all the above-mentioned scenarios, available space gaps were calculated based on the location of lead vehicle, length of lead vehicle, location of lag vehicle using Equation 5.11. The space gaps were converted into time gaps using the speed of lag vehicle using Equation 5.12 .

availableGap $_{\text {space }}=$ vehLoclead - vehLoclag - vehLen lead

Where

availableGap $_{\text {space }}=$ available space gap $(\mathrm{m})$

vehLoclead $=$ location of the lead vehicle $(\mathrm{m})$

$v_{\text {ehLoclag }}=$ location of the lag vehicle $(\mathrm{m})$

$v_{\text {ehLen }}$ lead $=$ length of the lead vehicle $(\mathrm{m})$

$$
\text { availableGap }_{\text {time }}=\frac{\text { avaialbleGap }_{\text {space }}}{\text { speed }_{\text {lag }}}
$$

Where

availableGap $_{\text {space }}=$ available space gap $(\mathrm{m})$

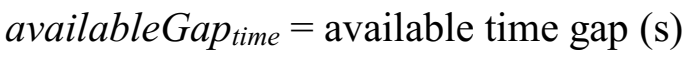

speed $_{\text {lag }}=$ speed of the lag vehicle $(\mathrm{m} / \mathrm{s})$ 


\subsubsection{PNC Evaluation - Method 1}

The start time and final time for possible merging by the SCL vehicle were calculated based on the speed and acceleration characteristics. After calculating the available gaps in FRL, the distances required for the SCL vehicle to merge at the all the available gaps were calculated. For each gap, three merging distances were calculated i.e., at the beginning, at the lagGapRatio and at the end of the gap assuming that the SCL vehicle can merge at any point in the available gap. As shown in Figure 5.3, the proportion of total available gap between the SCL vehicle and lag FRL vehicle is referred as lagGapRatio in this study. The author of this study had observed the variation in mean PNC values by dividing the total gap with different proportions of lead and lag gaps. It was found that the mean PNC was lowest when the total gap was distributed as $40 \%$ lag gap and $60 \%$ lead gap. Hence, the lagGapRatio was considered as 0.4 in this study. Relative distances were calculated corresponding to the merging locations of the SCL vehicle. Estimated times required for the SCL vehicle to reach the merging locations were also determined.

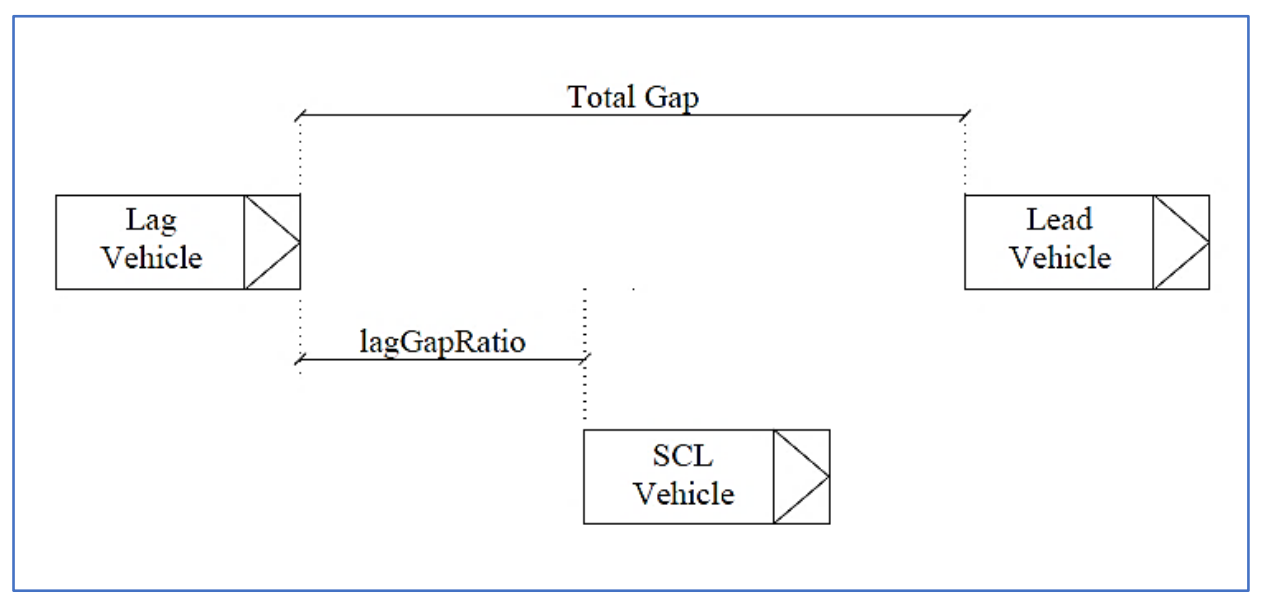

Figure 5.3: Illustration of lagGapRatio 
PNC Due to Acceleration Failure:

Acceleration rates of SCL vehicle corresponding to all distances, times to reach the merging locations and gore speed of the SCL vehicle were calculated using Equation 5.13. Using the Normal cumulative distribution function of the MATLAB 2018a, PNC due to acceleration failure for all the acceleration rates of SCL vehicle were calculated based on the mean and standard deviation of the acceleration rates.

$$
\text { accRate }_{S C L}=\frac{2 *(\text { distToMerge } / \text { timeToMerge }- \text { goreSpeed })}{\text { timeToMerge }}
$$

Where

accRatesCL acceleration rate of the SCL vehicle $\left(\mathrm{m} / \mathrm{s}^{2}\right)$

distToMerge $=$ distance from the merging location $(\mathrm{m})$

timeToMerge $=$ estimated time to reach the merging location (s)

goreSpeed $=$ gore speed of the SCL vehicle $(\mathrm{m} / \mathrm{s})$

PNC Due to Total Gap Failure:

Fatema et al. (2014) developed a model by studying the gap acceptance behaviour of the SCL drivers for predicting the total gap using the merge speed and relative distance of the merging location. The model was given by Equation 5.14 and the standard deviation of the model is 0.963 .

$$
\text { AcceptedGap }=9.563-(0.216 * \text { MergeSpeed })-(1.322 * \text { relDistance })
$$

where 
AcceptedGap $=$ accepted total gap of the SCL vehicle (s)

MergeSpeed $=$ merge speed of the SCL vehicle $(\mathrm{m} / \mathrm{s})$

relDistance $=$ relative distance of the merging location

Minimum accepted gaps for the SCL vehicle were also calculated using the SCL vehicle length and speeds of the FRL vehicles in the simulation limits and the minimum headway on FRL. Using the Normal cumulative distribution function of the MATLAB 2018a, PNC due to total gap failure at all the available time gaps was calculated based on the mean and standard deviation of the accepted total gap.

The combined PNC due to both gap failure and acceleration failure was calculated using the equation

$$
c o m b P N C=\text { gapFailPNC }+ \text { accFailPNC }-(\text { gapFailPNC*accFailPNC })
$$

Where

$c o m b P N C=$ combined PNC due to both acceleration and gap failure

gapFailPNC $=$ PNC due to total gap failure

accFailPNC $=$ PNC due to acceleration failure

Both failures due to acceleration and gap acceptance depend on the driver behaviour in the real world. A driver may sometimes decide to accept the smaller gaps or may decide to increase the acceleration beyond the allowable limits or both. Driver's decision to accept the smaller gaps might not imply that the driver has to increase the acceleration beyond the limits and vice versa. For the same reason, acceleration failure and gap acceptance failure 
can be considered as independent of each other, which may not be true all the time in real world. But in the simulation methodologies developed in this study, both the failures were considered as independent and the probability of one failure would not affect the probability of the other failure.

Final combined PNC of the SCL vehicle is the minimum of all the PNC values due to gap failure and acceleration failure. Output from the Method 1 is the mean PNC of all the SCL vehicles generated in the simulation which represents the mean probability of uncomfortable merging of all the SCL vehicles simulated in the absence of vehicle connectivity.

\subsubsection{PNC Evaluation - Method 2}

The second simulation technique, Method 2 is similar to the Method 1 till the calculation of available gaps on FRL as mentioned in Section 5.2. After calculating the available gaps in FRL, a single gap was targeted based on the mean merge speed and mean acceleration rate of the SCL vehicle. Based on the mean location of merging, lead vehicle which is ahead of SCL vehicle was selected. Depending on if the lead vehicle is within the simulation limits or not and the presence of lag vehicle within the simulation limits, the available time gap was calculated. If the available gap is greater than the acceptable maximum required gap which is 6 seconds in this study or if there is no lag vehicle present within the simulation limits, then the deceleration was not requested for lag vehicle. If the lag vehicle is the last vehicle on FRL within the simulation limits, then the lag vehicle can decelerate without any conditions. If the lag vehicle is not the last vehicle on FRL within the simulation limits, then the lag vehicle will decelerate only if the vehicle behind it has 
at least twice the minimum headway of the lag vehicle and has not reached the critical headway. After deciding about the cooperative merging options of the lag vehicle, final combined PNC due to gap failure and acceleration failure of the SCL vehicle at the targeted gap was calculated similar to the Method 1 procedure. Final output, mean combined PNC from this method quantifies the probability of forced merging of SCL vehicles in the presence of vehicle connectivity and cooperative merging was attempted for the lag vehicle on FRL.

\subsubsection{PNC Evaluation - Method 3}

PNC evaluation by Method 3 is similar to Method 2 until the identification of lead and lag vehicles in the FRL. Similar to the FRL vehicles, characteristics of F2L vehicles such as vehicle lengths, speeds, headways and critical headways were assigned. In this method, a lane changing request was issued for the lag vehicle and depending on the presence of other vehicles in the target lane which is F2L. Minimum headways to the lag and lead vehicles in the F2L were estimated. Different conditions that were examined before changing the lane of the lag vehicle are:

(i) If there is no lag vehicle in the F2L, then set the new location of the vehicle as the location in the FRL. The new location cannot be less than the old vehicle location and should maintain the minimum headway from the lead vehicle. Speed of the vehicle in the new lane was not changed and critical headway for the lead vehicle was calculated. Then the vehicle was added to the F2L and combined PNC of the SCL vehicle was calculated to merge at the gap created by lag vehicle FRL. 
(ii) If there is no lead vehicle in the F2L, then set the new location of the vehicle as equal to the location in the FRL. The new location cannot be less than the old vehicle location and should maintain the minimum headway from the lag vehicle. Speed of the vehicle in the new lane was not changed and critical headway for the lag vehicle was calculated. Then the vehicle was added to the F2L and combined PNC of the SCL vehicle was calculated to merge at the gap created by lag vehicle in FRL.

(iii) If both the lead and lag vehicles were present in the destination lane, then the minimum headways were calculated for both lead and lag vehicles. New location of the vehicle in F2L was positioned such that it should maintain minimum headway from its lead and lag vehicles. The new location of the vehicle in the F2L was calculated such that its speed cannot be less than the speed of its lag vehicle and cannot be greater than the speed of its lead vehicle. The new speed of the vehicle was calculated using the interpolation technique between the speeds of lag vehicle and lead vehicle. Critical headways for lead vehicle and lag vehicle were calculated and then the vehicle was added to the F2L. Combined PNC was calculated for the SCL vehicle to merge at the gap created by lag vehicle in FRL.

Final output, mean combined PNC from this method quantifies the probability of forced merging of SCL vehicles in the presence of vehicle connectivity and lane change request process was attempted for the lag vehicle on FRL.

All the results obtained from the application of above explained methodologies and the detailed analysis of the results are presented in the next chapter. 


\section{Chapter 6: ANALYSIS AND RESULTS}

Different simulation techniques to examine the effects of vehicle connectivity on improving the safety and efficiency of freeway on-ramps were explained in the previous chapter. Probability of non-compliance (PNC) is the outcome of all the methods, which measures the probability of uncomfortable or forced merging of speed change lane (SCL) drivers.

Section 6.1 presents the number of model runs required for all the methods to accurately represent the site conditions. Next section 6.2 reports the mean PNC and standard deviation of PNC at the selected 16 SCL sites on Highway 417 by applying the developed Methods 1, 2 and 3. Section 6.3 discusses the relationship between mean PNC and the collision frequency by means of a collision prediction model (CPM) using the statistical computing software package R version 3.5.1. Next, Section 6.4 explores the sensitivity of each selected input parameter in the application of developed methodologies. Proposed methodologies were applied to SCL lengths recommended by AASHTO (2011) and TAC (2017) to calculate the mean PNC and to check the consistency of the results, which will be explained in Section 6.5.

\subsection{Number of Runs Required}

It was required to establish the optimum number of model runs required to accurately represent the site conditions and vehicle volumes on freeway right lane (FRL) and SCL. A large number of vehicles needed to be simulated in order to maintain the consistency in the results and to account for the randomization effect of the numbers on the output. Therefore, a specific value was allocated to each input parameter based on the field data available and 
mean PNC was calculated for different number of runs. Based on the percentage change in mean PNC from different runs, the optimum number of runs was chosen. Each run represents that the vehicles were simulated for one hour.

Based on the minimum and maximum values, the distribution of input parameters as explained in Sections 3.2 and 3.3, values assumed for each input parameter are given below:

- SCL length: Minimum SCL length was $188 \mathrm{~m}$ and maximum SCL length was 468 $\mathrm{m}$. Almost half of the SCL lengths were more than $400 \mathrm{~m}$. Hence, the base value was chosen as $400 \mathrm{~m}$.

- SCL volume: Minimum SCL volume was $95 \mathrm{veh} / \mathrm{h}$ and maximum volume was 965 $\mathrm{veh} / \mathrm{h}$. Base value of SCL volume was chosen $400 \mathrm{veh} / \mathrm{h}$ to be close to average value.

- Mean FRL speed: Minimum FRL speed was $90.41 \mathrm{~km} / \mathrm{h}$ and maximum speed was $109.73 \mathrm{~km} / \mathrm{h}$. Average FRL speed $102.16 \mathrm{~km} / \mathrm{h}$ was taken as base value.

- Standard Deviation of FRL speed: Minimum SD of FRL speed was $7.29 \mathrm{~km} / \mathrm{h}$ and maximum was $11.75 \mathrm{~km} / \mathrm{h}$. Average SD of FRL speed $8.78 \mathrm{~km} / \mathrm{h}$ was taken as base value.

- FRL volume: Minimum FRL volume was $345 \mathrm{veh} / \mathrm{h}$ and maximum volume was $1244 \mathrm{veh} / \mathrm{h}$. Base value of SCL volume was chosen $800 \mathrm{veh} / \mathrm{h}$ to be close to average value.

- $85^{\text {th }}$ percentile gore speed: Minimum speed was $62.51 \mathrm{~km} / \mathrm{h}$ and maximum speed was $106.78 \mathrm{~km} / \mathrm{h}$. Base value of $85^{\text {th }}$ percentile gore speed was taken as $70 \mathrm{~km} / \mathrm{h}$. 
- Percentage of HV on FRL: Minimum percentage of HV on FRL was $5.1 \%$ and $20.2 \%$. The base value was considered as $10 \%$.

Output mean PNC values after running the simulations with above given values were given in the Table 6.1:

Table 6.1: Mean PNC Values for Different Number of Model Runs.

\begin{tabular}{|c|c|c|c|c|c|c|c|}
\hline \multirow{2}{*}{$\begin{array}{c}\text { Number } \\
\text { of Runs }\end{array}$} & \multirow{2}{*}{$\begin{array}{c}\text { Number } \\
\text { of SCL } \\
\text { Vehicles }\end{array}$} & $\begin{array}{c}\text { Mean } \\
\text { PNC }\end{array}$ & $\begin{array}{c}\text { Method 1 } \\
\text { Mean } \\
\text { PNC } \\
\text { i\%) }\end{array}$ & $\begin{array}{c}\text { Mean } \\
\text { PNC }\end{array}$ & $\begin{array}{c}\text { Change } \\
\text { in } \\
\text { Mean } \\
\text { PNC } \\
\text { (\%) }\end{array}$ & $\begin{array}{c}\text { Mean } \\
\text { PNC }\end{array}$ & $\begin{array}{c}\text { Change } \\
\text { in } \\
\text { Mean } \\
\text { PNC } \\
\mathbf{( \% )}\end{array}$ \\
\hline 10 & 4000 & 0.0595 & & 0.0558 & & 0.0535 & \multicolumn{2}{|c|}{} \\
\hline 25 & 10000 & 0.0561 & -5.74 & 0.0546 & -2.21 & 0.0527 & -1.37 \\
\hline 35 & 14000 & 0.0575 & 2.39 & 0.0531 & -2.66 & 0.0523 & -0.83 \\
\hline 50 & 20000 & 0.0577 & 1.44 & 0.0535 & 0.63 & 0.0543 & 3.78 \\
\hline 60 & 24000 & 0.0574 & -0.50 & 0.0516 & -3.47 & 0.0537 & -1.00 \\
\hline 65 & 26000 & 0.0579 & 0.79 & 0.0523 & 1.43 & 0.0515 & -4.08 \\
\hline 70 & 28000 & 0.0585 & 0.97 & 0.0533 & 1.80 & 0.0529 & 2.63 \\
\hline 75 & 30000 & 0.0583 & -0.27 & 0.0546 & 2.49 & 0.0546 & 3.23 \\
\hline 80 & 32000 & 0.0567 & -2.82 & 0.0533 & -2.39 & 0.0532 & -2.51 \\
\hline 85 & 34000 & 0.0574 & 1.25 & 0.0515 & -3.34 & 0.0527 & -0.94 \\
\hline 90 & 36000 & 0.0587 & 2.37 & 0.0526 & 2.02 & 0.0526 & -0.27 \\
\hline 95 & 38000 & 0.0595 & 1.29 & 0.0530 & 0.86 & 0.0532 & 1.06 \\
\hline 100 & 40000 & 0.0590 & -0.89 & 0.0525 & -0.98 & 0.0511 & -3.88 \\
\hline
\end{tabular}

At 60 simulation runs, the percentage change in the mean PNC was less than $1 \%$ in Method 1. At 50 simulation runs, the percentage change in the mean PNC was less than $1 \%$ in Method 2. At 35 simulation runs, the percentage change in the mean PNC was less than 
$1 \%$ in Method 3. To increase the accuracy of the simulation, maximum number of runs was considered from all methods. Hence, the optimum number of model runs was decided as 60 in this study.

\subsection{PNC at the Study SCL Locations}

Input parameters of all the 16 study sites were assigned and the model was simulated for 60 runs in all methods. The final output of the model indicates the mean and standard deviation of PNC for all simulated SCL vehicles. The mean and standard deviation values of PNC at the 16 sites evaluated using the developed three simulation techniques are shown in the Table 6.2. Percentage decrease in the mean PNC from method 1 to 2 and from method 1 to 3 is also listed in the same table. A few observations from the table are:

- Highest mean PNC values in Methods 1,2 and 3 were found at the site Innes (E-E) and the lowest values were found at the site Walkley (W-W).

- The lowest mean PNC at Walkley (W-W) could be because of the lowest FRL volume which is $345 \mathrm{veh} / \mathrm{h}$.

- The highest mean PNC at Innes (E-E) could not be attributed to a single parameter but can be attributed to the combination of several parameters. The site has relatively high freeway speed and low merge speed values when compared with the other sites and also relatively high FRL volume.

- Eagleson (S-E) has a mean PNC close to the highest mean PNC, which has the highest FRL volume of $1257 \mathrm{veh} / \mathrm{h}$.

- Maitland (NS-W) also got a relatively low PNC which is close to the lowest mean PNC for all methods which could be attributed to the longest SCL of $468 \mathrm{~m}$. 
Table 6.2: PNC Values at the Study SCL Sites.

\begin{tabular}{|l|c|c|c|c|c|c|c|c|}
\hline \multicolumn{1}{|c|}{ Study site } & \multicolumn{2}{|c|}{ Method 1 } & \multicolumn{2}{c|}{ Method 2 } & \multicolumn{2}{c|}{ Method 3 } & \multicolumn{2}{c|}{$\begin{array}{c}\text { Percentage } \\
\text { Decrease in Mean } \\
\text { PNC (\%) }\end{array}$} \\
\hline & $\begin{array}{c}\text { Mean } \\
\text { PNC }\end{array}$ & $\begin{array}{c}\text { sd } \\
\text { PNC }\end{array}$ & $\begin{array}{c}\text { Mean } \\
\text { PNC }\end{array}$ & $\begin{array}{c}\text { sd } \\
\text { PNC }\end{array}$ & $\begin{array}{c}\text { Mean } \\
\text { PNC }\end{array}$ & $\begin{array}{c}\text { sd } \\
\text { PNC }\end{array}$ & $\begin{array}{c}\text { Method } \\
\text { 1 to 2 }\end{array}$ & $\begin{array}{c}\text { Method } \\
\text { 1 to 3 }\end{array}$ \\
\hline Carp N-E & 0.151 & 0.141 & 0.148 & 0.135 & 0.146 & 0.134 & 2.03 & 3.26 \\
\hline Carp S-E & 0.387 & 0.198 & 0.380 & 0.193 & 0.373 & 0.187 & 1.99 & 3.69 \\
\hline Eagleson S-E & 0.481 & 0.261 & 0.479 & 0.261 & 0.466 & 0.258 & 0.57 & 3.24 \\
\hline Innes E-E & 0.489 & 0.214 & 0.480 & 0.208 & 0.473 & 0.207 & 1.78 & 3.14 \\
\hline Maitland NS-W & 0.066 & 0.161 & 0.058 & 0.145 & 0.063 & 0.154 & 12.52 & 4.50 \\
\hline Moodie N-W & 0.438 & 0.284 & 0.416 & 0.275 & 0.427 & 0.276 & 4.91 & 2.50 \\
\hline Parkdale NS-W & 0.203 & 0.263 & 0.189 & 0.247 & 0.184 & 0.246 & 6.78 & 9.59 \\
\hline Richmond S-E & 0.179 & 0.171 & 0.170 & 0.158 & 0.169 & 0.153 & 5.01 & 5.74 \\
\hline Terryfox N-E & 0.165 & 0.177 & 0.156 & 0.167 & 0.152 & 0.158 & 5.61 & 8.10 \\
\hline Terryfox N-W & 0.397 & 0.193 & 0.387 & 0.186 & 0.382 & 0.181 & 2.49 & 3.73 \\
\hline Terryfox S-E & 0.379 & 0.201 & 0.367 & 0.195 & 0.360 & 0.189 & 3.14 & 4.95 \\
\hline Terryfox S-W & 0.209 & 0.198 & 0.201 & 0.184 & 0.198 & 0.184 & 4.09 & 5.24 \\
\hline Vanier N-E & 0.231 & 0.271 & 0.216 & 0.257 & 0.208 & 0.253 & 6.64 & 10.06 \\
\hline Walkley W-W & 0.058 & 0.071 & 0.058 & 0.071 & 0.058 & 0.071 & 1.03 & 1.32 \\
\hline Woodroffe N-E & 0.296 & 0.318 & 0.268 & 0.297 & 0.260 & 0.295 & 9.18 & 12.14 \\
\hline Woodroffe NS-W & 0.211 & 0.247 & 0.192 & 0.227 & 0.187 & 0.224 & 8.91 & 10.98 \\
\hline
\end{tabular}

Along with the mean PNC from each method, PNC values of each simulated SCL vehicle were also obtained. From the detailed output of PNC values of each simulated SCL vehicle, the percentages of SCL vehicles with different ranges of PNC values were also determined. Table 6.3, Table 6.4, Table 6.5 display the percentages of SCL vehicles between specific 
ranges such as $0,>0.0$ to $0.2,>0.2$ to $0.4,>0.4$ to $0.6,>0.6$ to $0.8,>0.8$ to 1.0 from Methods 1, 2 and 3.

Table 6.3: Percentage Ranges of PNC Values - Method 1.

\begin{tabular}{|c|c|c|c|c|c|c|c|}
\hline SCL Site & $\begin{array}{l}\text { PNC } \\
=0.0\end{array}$ & $\begin{array}{c}\text { PNC } \\
>0.0 \text { to } \\
0.2\end{array}$ & $\begin{array}{c}\text { PNC } \\
>0.2 \text { to } \\
0.4\end{array}$ & $\begin{array}{c}\text { PNC } \\
>0.4 \text { to } \\
0.6\end{array}$ & $\begin{array}{c}\text { PNC } \\
>0.6 \text { to } \\
0.8\end{array}$ & $\begin{array}{c}\text { PNC } \\
>0.8 \text { to } \\
<1.0\end{array}$ & $\begin{array}{l}\text { PNC } \\
=1.0\end{array}$ \\
\hline & $(\%)$ & (\%) & (\%) & (\%) & (\%) & (\%) & (\%) \\
\hline Carp N-E & 0.00 & 79.79 & 14.60 & 3.08 & 1.45 & 1.07 & 0.00 \\
\hline Carp S-E & 0.00 & 17.51 & 42.06 & 26.19 & 9.32 & 4.67 & 0.25 \\
\hline Eagleson S-E & 0.00 & 13.35 & 33.33 & 22.89 & 12.72 & 17.13 & 0.58 \\
\hline Innes E-E & 0.00 & 6.69 & 32.22 & 32.49 & 18.09 & 9.92 & 0.59 \\
\hline Maitland NS-W & 0.00 & 91.39 & 3.37 & 2.01 & 1.71 & 1.52 & 0.00 \\
\hline Moodie N-W & 0.24 & 23.81 & 32.10 & 15.78 & 10.38 & 17.37 & 0.32 \\
\hline Parkdale NS-W & 0.00 & 74.36 & 10.36 & 3.98 & 3.31 & 7.95 & 0.04 \\
\hline Richmond S-E & 0.00 & 73.38 & 18.23 & 3.65 & 2.70 & 2.01 & 0.03 \\
\hline Terryfox N-E & 0.00 & 78.02 & 12.84 & 4.23 & 2.72 & 2.16 & 0.03 \\
\hline Terryfox N-W & 0.00 & 14.92 & 41.99 & 28.69 & 9.95 & 4.13 & 0.33 \\
\hline Terryfox S-E & 0.00 & 18.74 & 42.57 & 25.38 & 8.37 & 4.61 & 0.34 \\
\hline Terryfox S-W & 0.39 & 66.39 & 20.82 & 5.39 & 3.79 & 3.18 & 0.05 \\
\hline Vanier N-E & 0.00 & 67.38 & 12.03 & 7.28 & 5.48 & 7.82 & 0.01 \\
\hline Walkley W-W & 0.00 & 96.77 & 2.35 & 0.58 & 0.16 & 0.14 & 0.00 \\
\hline Woodroffe N-E & 0.00 & 61.13 & 11.43 & 6.67 & 6.48 & 14.26 & 0.03 \\
\hline Woodroffe NS-W & 0.00 & 71.68 & 11.59 & 5.75 & 4.84 & 6.13 & 0.01 \\
\hline
\end{tabular}

From the Table 6.3, it can be noticed that 10 out of the $16 \mathrm{SCL}$ sites have the major percentage of PNC values in the range of 0.0 to 0.2 which indicates the lower chances of uncomfortable merging for these percentages of vehicles. Rest of the SCL sites have the major percentage of PNC values in the range of $>0.2$ to 0.4 . The percentages of SCL vehicles having PNC equal to 1.0 are less than $1 \%$ at all the sites. 
Table 6.4: Percentage Ranges of PNC Values - Method 2.

\begin{tabular}{|c|c|c|c|c|c|c|c|}
\hline SCL Site & $\begin{array}{l}\text { PNC } \\
=0.0\end{array}$ & $\begin{array}{c}\text { PNC } \\
>0.0 \text { to } \\
0.2\end{array}$ & $\begin{array}{c}\text { PNC } \\
>0.2 \text { to } \\
0.4\end{array}$ & $\begin{array}{c}\text { PNC } \\
>0.4 \text { to } \\
0.6\end{array}$ & $\begin{array}{c}\text { PNC } \\
>0.6 \text { to } \\
0.8\end{array}$ & $\begin{array}{c}\text { PNC } \\
>0.8 \text { to } \\
<1.0\end{array}$ & $\begin{array}{l}\text { PNC } \\
=1.0\end{array}$ \\
\hline & $(\%)$ & $(\%)$ & $(\%)$ & $(\%)$ & $(\%)$ & $(\%)$ & $(\%)$ \\
\hline Carp N-E & 0.00 & 79.97 & 15.41 & 2.82 & 0.96 & 0.80 & 0.03 \\
\hline Carp S-E & 0.00 & 17.45 & 43.11 & 26.80 & 8.25 & 4.14 & 0.24 \\
\hline Eagleson S-E & 0.00 & 14.94 & 34.81 & 22.63 & 11.38 & 15.75 & 0.51 \\
\hline Innes E-E & 0.00 & 6.62 & 33.55 & 33.47 & 16.93 & 8.98 & 0.45 \\
\hline Maitland NS-W & 0.00 & 92.60 & 2.93 & 1.77 & 1.56 & 1.14 & 0.00 \\
\hline Moodie N-W & 0.02 & 24.86 & 34.86 & 15.36 & 9.58 & 14.91 & 0.40 \\
\hline Parkdale NS-W & 0.00 & 75.78 & 10.62 & 4.02 & 2.56 & 6.93 & 0.09 \\
\hline Richmond S-E & 0.00 & 74.71 & 18.39 & 3.43 & 1.69 & 1.75 & 0.02 \\
\hline Terryfox N-E & 0.00 & 79.26 & 13.16 & 3.64 & 2.19 & 1.74 & 0.00 \\
\hline Terryfox N-W & 0.00 & 15.18 & 44.18 & 27.85 & 8.77 & 3.77 & 0.26 \\
\hline Terryfox S-E & 0.00 & 20.16 & 43.82 & 24.37 & 7.31 & 4.01 & 0.33 \\
\hline Terryfox S-W & 0.00 & 67.07 & 22.11 & 5.32 & 2.79 & 2.70 & 0.02 \\
\hline Vanier N-E & 0.00 & 69.30 & 12.62 & 6.51 & 5.07 & 6.49 & 0.01 \\
\hline Walkley W-W & 0.00 & 96.88 & 2.23 & 0.56 & 0.20 & 0.13 & 0.00 \\
\hline Woodroffe N-E & 0.00 & 63.93 & 12.33 & 7.08 & 5.10 & 11.55 & 0.01 \\
\hline Woodroffe NS-W & 0.00 & 74.43 & 11.64 & 5.40 & 3.65 & 4.87 & 0.01 \\
\hline
\end{tabular}

From Table 6.4, it can be noticed that the 10 out of the 16 SCL sites have the major percentage of PNC values in the range of 0.0 to 0.2 which indicates the lower chances of uncomfortable merging for these percentages of vehicles. The rest of SCL sites have the major percentage of PNC values in the range of $>0.2$ to 0.4 . The percentages of SCL vehicles having PNC equal to 1.0 are less than $1 \%$ at all the sites. The same trend was observed in Method 1, but there is a slight increase in the percentages of vehicles with the PNC between 0.0 to 0.2 and there is also a slight decrease in the percentages of vehicles having the PNC between $>0.8$ to 1.0 and PNC equal to 1.0. This indicates a slight decrease in the number of vehicles with probability of uncomfortable merging. 
Table 6.5: Percentage Ranges of PNC Values - Method 3.

\begin{tabular}{|c|c|c|c|c|c|c|c|}
\hline SCL Site & $\begin{array}{l}\text { PNC } \\
=0.0\end{array}$ & $\begin{array}{c}\text { PNC } \\
>0.0 \text { to } \\
0.2\end{array}$ & $\begin{array}{c}\text { PNC } \\
>0.2 \text { to } \\
0.4\end{array}$ & $\begin{array}{c}\text { PNC } \\
>0.4 \text { to } \\
0.6\end{array}$ & $\begin{array}{c}\text { PNC } \\
>0.6 \text { to } \\
0.8\end{array}$ & $\begin{array}{c}\text { PNC } \\
>0.8 \text { to } \\
<1.0\end{array}$ & $\begin{array}{l}\text { PNC } \\
=1.0\end{array}$ \\
\hline & $(\%)$ & $(\%)$ & $(\%)$ & $(\%)$ & $(\%)$ & $(\%)$ & $(\%)$ \\
\hline Carp N-E & 0.07 & 80.70 & 14.40 & 2.75 & 1.13 & 0.94 & 0.02 \\
\hline Carp S-E & 0.00 & 17.68 & 44.34 & 26.56 & 7.66 & 3.59 & 0.16 \\
\hline Eagleson S-E & 0.00 & 14.44 & 33.87 & 23.08 & 12.37 & 15.66 & 0.57 \\
\hline Innes E-E & 0.00 & 7.08 & 34.32 & 33.23 & 16.55 & 8.38 & 0.42 \\
\hline Maitland NS-W & 0.00 & 92.07 & 3.04 & 1.98 & 1.52 & 1.38 & 0.00 \\
\hline Moodie N-W & 0.05 & 23.81 & 33.80 & 16.07 & 10.54 & 15.42 & 0.32 \\
\hline Parkdale NS-W & 0.00 & 78.59 & 9.91 & 3.35 & 2.24 & 5.84 & 0.07 \\
\hline Richmond S-E & 0.00 & 74.69 & 18.75 & 3.28 & 1.82 & 1.43 & 0.02 \\
\hline Terryfox N-E & 0.00 & 79.75 & 12.41 & 3.83 & 2.32 & 1.67 & 0.02 \\
\hline Terryfox N-W & 0.00 & 16.07 & 42.78 & 28.63 & 8.83 & 3.44 & 0.25 \\
\hline Terryfox S-E & 0.00 & 20.32 & 44.67 & 24.70 & 6.46 & 3.53 & 0.32 \\
\hline Terryfox S-W & 0.00 & 68.44 & 20.63 & 5.39 & 3.02 & 2.47 & 0.05 \\
\hline Vanier N-E & 0.00 & 71.02 & 11.85 & 6.42 & 4.33 & 6.37 & 0.01 \\
\hline Walkley W-W & 0.00 & 96.92 & 2.20 & 0.52 & 0.21 & 0.14 & 0.00 \\
\hline Woodroffe N-E & 0.00 & 65.53 & 12.07 & 5.99 & 5.47 & 10.90 & 0.04 \\
\hline Woodroffe NS-W & 0.00 & 75.23 & 11.19 & 5.24 & 3.76 & 4.56 & 0.01 \\
\hline
\end{tabular}

From Table 6.5, it can be observed that the 10 out of the 16 SCL sites have the major percentage of PNC values in the range of 0.0 to 0.2 which indicates the lower chances of uncomfortable merging for these percentages of vehicles. Rest of the SCL sites have the major percentage of PNC values in the range of $>0.2$ to 0.4 . The percentages of SCL vehicles having PNC equal to 1.0 are less than $1 \%$ at all the sites. The same trend was observed in Method 1, but there is a slight increase in the percentages of vehicles with the PNC between 0.0 to 0.2 and there is also a slight decrease in the percentages of vehicles having the PNC between $>0.8$ to 1.0 and PNC equal to 1.0 . This indicates a slight decrease in the number of vehicles with probability of uncomfortable merging which is a similar decrease from Method 1 to Method 2. 
From the above results, it can be noticed that there was not only a decrease in the mean PNC in Method 2 and 3, but also the percentages of vehicles with higher range of PNC were also decreased from Method 1 to Method 2 and 3.

\subsection{Relationship Between PNC and Collision Frequency}

In this section, an attempt was made to identify the relationship between mean PNC and the collision frequency. Safety performance of the freeways in influenced by the parameters like traffic volume and length of SCL. Regression models that relate these parameters to the collision frequency would serve as important tools to optimize the safety performance. Such models would be helpful in evaluating an existing design or designing a new roadway facility. Sayed and Leur (2008) mentioned that a foundation model for the collision prediction will have the traffic volume and the road length as independent variables and the collision frequency as the dependent variable. Collision data, AADT values for freeway through lanes and SCL were obtained as explained in Sections 3.2.6 and 3.2.7. Different collision prediction models (CPMs) were examined considering the traffic volumes on FRL and SCL, SCL length as independent variables. Exposure for the road segments is commonly expressed in million vehicle-kilometers for the period of collisions data collected and is calculated as given by Equation 6.1.

$$
\operatorname{Exp}=N * Q * 365 * L * 10^{-9}
$$

Where

Exp $=$ exposure in million vehicle-kilometers

$N=$ number of years of collisions data

$Q=$ average annual daily traffic (veh/day) 
$L=$ length of the SCL (m)

Table 6.6: Collision Prediction Models with AADT and SCL Length.

\begin{tabular}{|c|c|c|}
\hline Model No. & Model Equation & AIC \\
\hline $\mathrm{CPM}_{1}$ & $0.593^{\dagger}-0.000004 \times L^{\dagger}+0.00002 \times Q_{E n}^{\dagger}$ & 68.96 \\
\hline $\mathrm{CPM}_{2}$ & $-0.917^{\dagger}-0.0019 \times L^{\dagger}+0.00002 \times Q_{T h}^{\dagger}$ & 65.86 \\
\hline $\mathrm{CPM}_{3}$ & $-0.720^{\dagger}-0.0013 \times L^{\dagger}+0.00002 \times Q_{T h E n}^{\dagger}$ & 66.26 \\
\hline $\mathrm{CPM}_{4}$ & $-1.121^{\dagger}-0.0017 \times L^{\dagger}+0.00002 \times Q_{\text {Tot }}^{*}$ & 63.76 \\
\hline $\mathrm{CPM}_{5}$ & $0.520^{\dagger}+0.049 \times \operatorname{Exp}_{E n}^{\dagger}$ & 66.82 \\
\hline $\mathrm{CPM}_{6}$ & $-0.187^{\dagger}+0.038 \times E x p_{T h}^{*}$ & 63.04 \\
\hline $\mathrm{CPM}_{7}$ & $-0.171^{\dagger}+0.032 \times \operatorname{Exp}_{T h E n}^{\dagger}$ & 63.58 \\
\hline $\mathrm{CPM}_{8}$ & $-0.325^{*}+0.034 \times \operatorname{Exp}_{T o t}^{*}$ & 62.31 \\
\hline $\mathrm{CPM}_{9}$ & $-0.017^{\dagger}-0.152 \times \log (L)^{\dagger}+0.192 \times \log \left(Q_{E n}\right)^{\dagger}$ & 68.84 \\
\hline $\mathrm{CPM}_{10}$ & $-13.687^{\dagger}+0.811 \times \log (L)^{\dagger}+0.931 \times \log \left(Q_{T h}\right)^{*}$ & 65.35 \\
\hline $\mathrm{CPM}_{11}$ & $-11.484^{\dagger}+0.544 \times \log (L)^{\dagger}+0.857 \times \log \left(Q_{T h E n}\right)^{\dagger}$ & 66.35 \\
\hline $\mathrm{CPM}_{12}$ & $-16.849^{\dagger}+0.848 \times \log (L)^{\dagger}+1.184 \times \log \left(Q_{\text {Tot }}\right)^{*}$ & 64.09 \\
\hline $\mathrm{CPM}_{13}$ & $0.562^{\dagger}+0.129 \times \log \left(E x p_{E n}\right)^{\dagger}$ & 66.89 \\
\hline $\mathrm{CPM}_{14}$ & $-2.098^{\dagger}+0.933 \times \log \left(\operatorname{Exp}_{T h}\right)^{*}$ & 63.35 \\
\hline $\mathrm{CPM}_{15}$ & $-1.952^{\dagger}+0.841 \times \log \left(\operatorname{Exp}_{T h E n}\right)^{\dagger}$ & 64.42 \\
\hline $\mathrm{CPM}_{16}$ & $-3.132^{*}+1.171 \times \log \left(E_{x p} p_{T o t}\right)^{*}$ & 62.15 \\
\hline $\mathrm{CPM}_{17}$ & $-0.769^{\dagger}-0.0002 \times L^{\dagger}+0.184 \times \log \left(Q_{E n}\right)^{\dagger}$ & 68.85 \\
\hline $\mathrm{CPM}_{18}$ & $-9.613^{\dagger}+0.0024 \times L^{\dagger}+0.912 \times \log \left(Q_{T h}\right)^{*}$ & 65.36 \\
\hline $\mathrm{CPM}_{19}$ & $-8.745^{\dagger}+0.0016 \times L^{\dagger}+0.843 \times \log \left(Q_{T h E n}\right)^{\dagger}$ & 66.35 \\
\hline $\mathrm{CPM}_{20}$ & $-12.654^{*}+0.0026 \times L^{\dagger}+1.168 \times \log \left(Q_{\text {Tot }}\right)^{*}$ & 64.08 \\
\hline $\mathrm{CPM}_{21}$ & $1.204^{\dagger}+0.00002 \times Q_{E n}^{\dagger}+0.107 \times \log (L)^{\dagger}$ & 68.95 \\
\hline $\mathrm{CPM}_{22}$ & $-4.146^{\dagger}+0.00002 \times Q_{T h}^{\dagger}+0.663 \times \log (L)^{\dagger}$ & 65.83 \\
\hline $\mathrm{CPM}_{23}$ & $-3.055^{\dagger}+0.00002 \times Q_{T h E n}^{\dagger}+0.479 \times \log (L)^{\dagger}$ & 66.24 \\
\hline $\mathrm{CPM}_{24}$ & $-3.998^{\dagger}+0.00003 \times Q_{T o t}^{*}+5.939 \times \log (L)^{\dagger}$ & 64.79 \\
\hline
\end{tabular}


Where

$\mathrm{CPM}=$ collision prediction model

$L=$ length of the SCL (m)

$Q_{E n}=\mathrm{AADT}$ on entrance SCL (veh/day)

$Q_{T h}=\mathrm{AADT}$ on through lanes of the freeway (veh/day)

$Q_{T h E n}=$ sum of AADT on entrance SCL and through lanes (veh/day)

$Q_{T o t}=$ sum of AADT on entrance SCL, through lanes and exit SCL (veh/day)

$\operatorname{Exp}_{E n}=$ exposure term considering AADT for entrance SCL (million vehicle-kilometers)

$\operatorname{Exp}_{T h}=$ exposure term considering $\mathrm{AADT}$ for through lanes of the freeway (million vehicle-kilometers)

$\operatorname{Exp}_{T h E n}=$ exposure term considering the sum of AADT on entrance SCL and through lanes (million vehicle-kilometers)

$\operatorname{Exp}_{T o t}=$ exposure term considering sum of AADT on entrance SCL, through lanes and exit SCL (million vehicle-kilometers)

Table 6.6 shows various models that were developed relating the collision frequency to the AADT and SCL length. Based on the AIC value of the models, $\mathrm{CPM}_{16}$ was chosen as the appropriate model relating the input parameters to collision frequency.

Similar attempts were made to relate the collision frequency to the mean PNC along with traffic volume and SCL length. To all the CPMs mentioned in Table 6.6, mean PNC was 
also added as an independent variable and new CPMs were developed using negative binomial regression function of the software R (version 3.5.1) and presented by Table 6.7. It can be observed that including mean PNC as independent variable has decreased the AIC value indicating a better prediction of the collision frequency.

Table 6.7: Collision Prediction Models with AADT, SCL Length and Mean PNC.

\begin{tabular}{|c|l|c|}
\hline $\begin{array}{c}\text { Model } \\
\text { No. }\end{array}$ & \multicolumn{1}{|c|}{ Model Equation } & AIC \\
\hline $\mathrm{CPM}_{1}$ & $0.251^{\dagger}+0.00018 \times L^{\dagger}+0.000038 \times Q_{E n}^{\dagger}+0.673 \times P^{\dagger}$ & 70.89 \\
\hline $\mathrm{CPM}_{2}$ & $-5.413^{*}+0.00686 \times L^{\dagger}+0.000055 \times Q_{T h}^{* *}+5.719 \times P^{*}$ & 63.24 \\
\hline $\mathrm{CPM}_{3}$ & $-5.135^{*}+0.0060 \times L^{\dagger}+0.000049 \times Q_{T h E n}^{* *}+5.651 \times P^{*}$ & 63.75 \\
\hline $\mathrm{CPM}_{4}$ & $-5.919^{*}+0.0066 \times L^{*}+0.000054 \times Q_{T o t}^{* *}+6.007 \times P^{*}$ & 60.77 \\
\hline $\mathrm{CPM}_{5}$ & $0.043^{\dagger}+0.092 \times E x p_{E n}^{\dagger}+1.193 \times P^{\dagger}$ & 68.61 \\
\hline $\mathrm{CPM}_{6}$ & $-2.477^{*}+0.073 \times E x p_{T h}^{* *}+5.173 \times P^{*}$ & 60.16 \\
\hline $\mathrm{CPM}_{7}$ & $-2.539^{*}+0.066 \times E x p_{T h E n}^{* *}+5.218 \times P^{*}$ & 60.54 \\
\hline $\mathrm{CPM}_{8}$ & $-2.634^{*}+0.064 \times E x p_{T o t}^{* *}+4.984 \times P^{*}$ & 58.28 \\
\hline $\mathrm{CPM}_{9}$ & $-1.315^{\dagger}+0.751 \times P^{\dagger}-0.079 \times \log (L)^{\dagger}+0.272 \times \log \left(Q_{E n}\right)^{\dagger}$ & 70.74 \\
\hline $\mathrm{CPM}_{10}$ & $-29.868^{*}+4.486 \times P^{\dagger}+2.045 \times \log (L)^{\dagger}+1.673 \times \log \left(Q_{T h}\right)^{* *}$ & 63.85 \\
\hline $\mathrm{CPM}_{11}$ & $-28.432^{*}+4.479 \times P^{\dagger}+1.744 \times \log (L)^{\dagger}+1.681 \times \log \left(Q_{T h E n}\right)^{*}$ & 65.07 \\
\hline $\mathrm{CPM}_{12}$ & $-33.123^{* *}+4.267 \times P^{*}+1.985 \times \log (L)^{\dagger}+1.974 \times \log \left(Q_{T o t}\right)^{* *}$ & 61.99 \\
\hline $\mathrm{CPM}_{13}$ & $0.279^{\dagger}+0.750 \times P^{\dagger}+0.207 \times \log \left(\operatorname{Exp}_{E n}\right)^{\dagger}$ & 68.79 \\
\hline $\mathrm{CPM}_{14}$ & $-5.453^{*}+4.272 \times P^{\dagger}+1.651 \times \log \left(E x p_{T h}\right)^{* *}$ & 62.02 \\
\hline $\mathrm{CPM}_{15}$ & $-5.814^{*}+4.420 \times P^{\dagger}+1.677 \times \log \left(E x p_{T h E n}\right)^{*}$ & 63.44 \\
\hline $\mathrm{CPM}_{16}$ & $-6.972^{* *}+4.231 \times P^{*}+1.977 \times \log \left(E x p_{T o t}\right)^{* *}$ & \\
\hline $\mathrm{CPM}_{17}$ & $-1.764^{\dagger}+0.00012 \times L^{\dagger}+0.796 \times P^{\dagger}+0.263 \times \log \left(Q_{E n}\right)^{\dagger}$ & \\
\hline $\mathrm{CPM}_{18}$ & $-20.751^{* *}+0.007 \times L^{\dagger}+4.838 \times P^{*}+1.703 \times \log \left(Q_{T h}\right)^{* *}$ & \\
\hline $\mathrm{CPM}_{19}$ & $-20.647^{*}+0.006 \times L^{\dagger}+4.767 \times P^{*}+1.706 \times \log \left(Q_{T h E n}\right)^{*}$ & \\
\hline
\end{tabular}




\begin{tabular}{|c|c|c|}
\hline $\mathrm{CPM}_{20}$ & $-25.046^{* *}+0.007 \times L^{*}+4.759 \times P^{*}+2.059 \times \log \left(Q_{T o t}\right)^{* *}$ & 61.24 \\
\hline $\mathrm{CPM}_{21}$ & $0.713^{\dagger}+0.00004 \times Q_{E n}^{\dagger}+0.636 \times P^{\dagger}-0.068 \times \log (L)^{\dagger}$ & 70.89 \\
\hline $\mathrm{CPM}_{22}$ & $-15.520^{\dagger}+0.00005 \times Q_{T h}^{* *}+5.480 \times P^{*}+2.157 \times \log (L)^{\dagger}$ & 63.45 \\
\hline $\mathrm{CPM}_{23}$ & $-13.90^{\dagger}+0.00005 \times Q_{T h E n}^{* *}+5.444 \times P^{*}+1.874 \times \log (L)^{\dagger}$ & 63.94 \\
\hline $\mathrm{CPM}_{24}$ & $-14.850^{*}+0.00005 \times Q_{T o t}^{* *}+5.589 \times P^{*}+1.961 \times \log (L)^{\dagger}$ & 61.29 \\
\hline $\mathrm{CPM}_{25}$ & $0.596^{\dagger}-0.00014 \times L^{\dagger}+0.000011 \times Q_{E n}^{\dagger}-0.061 \times \log (P)^{\dagger}$ & 70.95 \\
\hline $\mathrm{CPM}_{26}$ & $-2.40^{\dagger}+0.0077 \times L^{\dagger}+0.000053 \times Q_{T h}^{* *}+1.132 \times \log (P)^{\dagger}$ & 64.89 \\
\hline $\mathrm{CPM}_{27}$ & $-2.201^{\dagger}+0.0069 \times L^{\dagger}+0.000049 \times Q_{T h E n}^{*}+1.146 \times \log (P)^{\dagger}$ & 65.20 \\
\hline $\mathrm{CPM}_{28}$ & $-2.890^{\dagger}+0.0081 \times L^{\dagger}+0.000056 \times Q_{T o t}^{* *}+1.348 \times \log (P)^{*}$ & 62.25 \\
\hline $\mathrm{CPM}_{29}$ & $0.603^{\dagger}+0.074 \times \operatorname{Exp}_{E n}^{\dagger}+0.113 \times \log (P)^{\dagger}$ & 68.79 \\
\hline $\mathrm{CPM}_{30}$ & $0.520^{\dagger}+0.078 \times E x p_{T h}^{* *}+1.149 \times \log (P)^{\dagger}$ & 61.26 \\
\hline $\mathrm{CPM}_{31}$ & $0.514^{\dagger}+0.074 \times \operatorname{Exp}_{T h E n}^{* *}+1.233 \times \log (P)^{*}$ & 61.25 \\
\hline $\mathrm{CPM}_{32}$ & $0.335^{\dagger}+0.073 \times \operatorname{Exp}_{T o t}^{* *}+1.280 \times \log (P)^{*}$ & 58.83 \\
\hline $\mathrm{CPM}_{33}$ & $0.061^{\dagger}-0.159 \times \log (L)^{\dagger}+0.187 \times \log \left(Q_{E n}\right)^{\dagger}-0.0099 \times \log (P)^{\dagger}$ & 70.84 \\
\hline $\mathrm{CPM}_{34}$ & $-27.33^{*}+2.186 \times \log (L)^{\dagger}+1.586 \times \log \left(Q_{T h}\right)^{*}+0.833 \times \log (P)^{\dagger}$ & 65.22 \\
\hline $\mathrm{CPM}_{35}$ & $-26.28^{\dagger}+1.931 \times \log (L)^{\dagger}+1.608 \times \log \left(Q_{T h E n}\right)^{*}+0.848 \times \log (P)^{\dagger}$ & 66.22 \\
\hline $\mathrm{CPM}_{36}$ & $-32.082^{*}+2.259 \times \log (L)^{\dagger}+1.959 \times \log \left(Q_{T o t}\right)^{* *}+0.884 \times \log (P)^{\dagger}$ & 63.08 \\
\hline $\mathrm{CPM}_{37}$ & $0.564^{\dagger}+0.131 \times \log \left(\operatorname{Exp}_{E n}\right)^{\dagger}+0.002 \times \log (P)^{\dagger}$ & 68.89 \\
\hline $\mathrm{CPM}_{38}$ & $-2.754^{\dagger}+1.487 \times \log \left(\operatorname{Exp}_{T h}\right)^{*}+0.691 \times \log (P)^{\dagger}$ & 63.53 \\
\hline $\mathrm{CPM}_{39}$ & $-3.097^{\dagger}+1.567 \times \log \left(\operatorname{Exp}_{T h E n}\right)^{*}+0.778 \times \log (P)^{\dagger}$ & 64.33 \\
\hline $\mathrm{CPM}_{40}$ & $-4.468^{*}+1.944 \times \log \left(\operatorname{Exp}_{\text {Tot }}\right)^{* *}+0.829 \times \log (P)^{\dagger}$ & 61.14 \\
\hline $\mathrm{CPM}_{41}$ & $-0.776^{\dagger}-0.00019 \times L^{\dagger}+0.185 \times \log \left(Q_{E n}\right)^{\dagger}+0.0015 \times \log (P)^{\dagger}$ & 70.85 \\
\hline $\mathrm{CPM}_{42}$ & $-17.561^{*}-0.007 \times L^{\dagger}+1.636 \times \log \left(Q_{T h}\right)^{*}+0.942 \times \log (P)^{\dagger}$ & 64.88 \\
\hline $\mathrm{CPM}_{43}$ & $-17.595^{*}-0.006 \times L^{\dagger}+1.647 \times \log \left(Q_{T h E n}\right)^{*}+0.937 \times \log (P)^{\dagger}$ & 65.93 \\
\hline
\end{tabular}




\begin{tabular}{|l|l|c|}
\hline $\mathrm{CPM}_{44}$ & $-22.716^{* *}-0.008 \times L^{\dagger}+2.070 \times \log \left(Q_{\text {Tot }}\right)^{* *}+1.035 \times \log (P)^{\dagger}$ & 62.37 \\
\hline $\mathrm{CPM}_{45}$ & $1.393^{\dagger}+0.000012 \times Q_{E n}^{\dagger}-1.476 \times \log (L)^{\dagger}-0.072 \times \log (P)^{\dagger}$ & 70.94 \\
\hline $\mathrm{CPM}_{46}$ & $-13.81^{\dagger}+0.000053 \times Q_{T h}^{* *}+2.403 \times \log (L)^{\dagger}+1.060 \times \log (P)^{\dagger}$ & 65.06 \\
\hline $\mathrm{CPM}_{47}$ & $-12.49^{\dagger}+0.000049 \times Q_{T h E n}^{*}+2.170 \times \log (L)^{\dagger}+1.080 \times \log (P)^{\dagger}$ & 65.36 \\
\hline $\mathrm{CPM}_{48}$ & $-13.94^{\dagger}+0.000054 \times Q_{T o t}^{* *}+2.368 \times \log (L)^{\dagger}+1.204 \times \log (P)^{\dagger}$ & 62.74 \\
\hline \multicolumn{2}{|l}{ Note: $\uparrow>0.05 ; *<0.05 ; * *<0.01 ; * * * 0.001 ;$} \\
\hline
\end{tabular}

After observing the AIC values of all the 48 CPMs from Table 6.7, the model $\mathrm{CPM}_{8}$ was chosen as a best possible model for collision prediction. All the input parameters of the model $\mathrm{CPM}_{8}$ were found to be significant at the $5 \%$ level of significance. Hence, the model relating the collision frequency to mean PNC was found as given by Equation 6.2.

$$
Y=e^{-2.634+0.064 \times E x p_{T o t}+4.984 \times P}
$$

Where

$\mathrm{Y}=$ collision frequency on acceleration lanes for the 5 years period

$P=$ mean PNC from Method 1

Having decided on the appropriate CPM, now an attempt was made to calculate the collision frequencies resulting from Methods 1,2 and 3 as shown in Table 6.8. It was observed that there is a decrease in the collision frequency at all the SCL sites from Method 1 to Method 2 and 3 except at one site. These results can be related to the percentage change in mean PNC from Method 1 to 2 and from Method 1 to 3 as mentioned in Table 6.2. SCL sites with relatively high percentage decrease in the mean PNC have resulted in a decrease in the collision frequency. Hence, it can be said that the proposed simulation methods are 
effective in reducing the collision frequencies if the percentage change in the mean PNC is high among the proposed methodologies.

Table 6.8: Predicted Collision Frequencies from the CPM.

\begin{tabular}{|c|c|c|c|c|c|c|c|}
\hline \multirow{2}{*}{ SCL Site } & \multirow{2}{*}{ Exposure } & \multicolumn{3}{|c|}{ Mean PNC } & \multicolumn{3}{c|}{ Collision Frequency } \\
\cline { 3 - 8 } & & $\mathbf{M}_{\mathbf{1}}$ & $\mathbf{M}_{\mathbf{2}}$ & $\mathbf{M}_{\mathbf{3}}$ & $\mathbf{M}_{\mathbf{1}}$ & $\mathbf{M}_{\mathbf{2}}$ & $\mathbf{M}_{\mathbf{3}}$ \\
\hline Carp N-E & 14.04 & 0.151 & 0.148 & 0.146 & 0.374 & 0.369 & 0.365 \\
\hline Carp S-E & 17.14 & 0.387 & 0.380 & 0.373 & 1.480 & 1.429 & 1.380 \\
\hline Eagleson S-E & 23.02 & 0.481 & 0.479 & 0.466 & 3.444 & 3.410 & 3.196 \\
\hline Innes E-E & 16.15 & 0.489 & 0.480 & 0.473 & 2.309 & 2.208 & 2.132 \\
\hline Maitland NS-W & 66.21 & 0.066 & 0.058 & 0.063 & 6.908 & 6.638 & 6.806 \\
\hline Moodie N-W & 22.80 & 0.438 & 0.416 & 0.427 & 2.741 & 2.457 & 2.595 \\
\hline Parkdale NS-W & 26.03 & 0.203 & 0.189 & 0.184 & 1.045 & 0.974 & 0.950 \\
\hline Richmond S-E & 33.00 & 0.179 & 0.170 & 0.169 & 1.448 & 1.385 & 1.378 \\
\hline Terryfox N-E & 16.58 & 0.165 & 0.156 & 0.152 & 0.472 & 0.451 & 0.443 \\
\hline Terryfox N-W & 13.80 & 0.397 & 0.387 & 0.382 & 1.256 & 1.195 & 1.166 \\
\hline Terryfox S-E & 21.91 & 0.379 & 0.367 & 0.360 & 1.929 & 1.817 & 1.755 \\
\hline Terryfox S-W & 11.76 & 0.209 & 0.201 & 0.198 & 0.432 & 0.415 & 0.409 \\
\hline Vanier N-E & 42.97 & 0.231 & 0.216 & 0.208 & 3.552 & 3.296 & 3.167 \\
\hline Walkley W_W & 20.42 & 0.058 & 0.058 & 0.058 & 0.354 & 0.354 & 0.354 \\
\hline Woodroffe N-E & 24.64 & 0.296 & 0.268 & 0.260 & 1.519 & 1.321 & 1.270 \\
\hline Woodroffe NS-W & 40.09 & 0.211 & 0.192 & 0.187 & 2.673 & 2.431 & 2.372 \\
\hline
\end{tabular}

After observing the reduction in collision frequency from Method 1 to Method 2 and 3, now it is required to identify which parameters were responsible for these results. Some of the parameters might significantly impact the output and some of them might not. It is important to know which parameters are to be given high importance in the design so that maximum benefits could be obtained from the designed facility. Next section conducts the sensitivity analysis of the selected input parameters to observe the effects of changes in the selected parameters on the output PNC results. 


\subsection{Sensitivity Analysis}

Effect of changes in the selected parameters was observed through the method of sensitivity analysis. One input parameter will be varied while keeping the other parameters as constants to examine the sensitivity of the varied parameter. The parameters selected for this process are gore speed, FRL speed, F2L speed, FRL traffic volume, lane ratio, SCL traffic volume, SCL length, percentage of HV on FRL and percentage of HV on F2L. Base values for these parameters were assumed based on the distributions of these parameters analysed from the field data and the range of minimum and maximum values for the same parameters as explained in the Section 6.1. These parameters were decreased and increased by $10 \%, 20 \%, 30 \%$ from the base value to verify the sensitivity of each parameter on the mean PNC from all the methods as presented in the Table 6.9.

Table 6.9: Input Parameters for Sensitivity Analysis.

\begin{tabular}{|l|c|c|c|c|c|c|c|}
\hline \multirow{2}{*}{\multicolumn{1}{|c|}{ Parameter }} & \multicolumn{7}{|c|}{ Change in Parameter } \\
\cline { 2 - 9 } & $\mathbf{- 3 0 \%}$ & $\mathbf{- 2 0 \%}$ & $\mathbf{- 1 0 \%}$ & Base & $\mathbf{+ 1 0 \%}$ & $\mathbf{+ 2 0 \%}$ & $\mathbf{+ 3 0 \%}$ \\
\hline Gore speed $(\mathrm{km} / \mathrm{h})$ & 49.0 & 56.0 & 63.0 & 70.0 & 77.0 & 84.0 & 91.0 \\
\hline FRL speed $(\mathrm{km} / \mathrm{h})$ & 71.51 & 81.73 & 91.94 & 102.16 & 112.38 & 122.59 & 132.81 \\
\hline FRL traffic volume $(\mathrm{veh} / \mathrm{h})$ & 560 & 640 & 720 & 800 & 880 & 960 & 1040 \\
\hline Lane ratio & 1.05 & 1.20 & 1.35 & 1.50 & 1.65 & 1.80 & 1.95 \\
\hline SCL traffic volume $(\mathrm{veh} / \mathrm{h})$ & 280 & 320 & 360 & 400 & 440 & 480 & 520 \\
\hline SCL length $(\mathrm{m})$ & 280 & 320 & 360 & 400 & 440 & 480 & 520 \\
\hline
\end{tabular}

Mean PNC values from Methods 1, 2 and 3 after using the input parameters from Table 6.9, are provided in the following sections along with the percentage changes in mean PNC and the key observations from the results. 


\subsubsection{Effect of Change in Gore Speed}

Table 6.10 displays the mean PNC values and percentage changes in the mean PNC values from Methods 1, 2 and 3 for different values of gore speed. These percentage changes were calculated from the base value. Figure 6.1 shows the mean PNC values for the change in gore speed and Figure 6.2 shows the changes in mean PNC for the change in gore speed. Mean PNC increased with increase in the gore speed and vice versa. The same trend was observed in all three methods and it should be noticed that the mean PNC was decreased from Method 1 to Method 2 and from Method 1 to Method 3. When the gore speed of the SCL drivers is high, they have less time and distance to complete merging action before they reach the end of SCL and they might tend to accept the shorter gaps which increases the probability of uncomfortable merging.

Table 6.10: Mean PNC and Change in Mean PNC for the Change in Gore Speed.

\begin{tabular}{|c|c|c|c|c|c|c|c|}
\hline \multicolumn{8}{|c|}{ Gore Speed (km/h) } \\
\hline \multirow{2}{*}{$\begin{array}{c}\text { Change in } \\
\text { Parameter } \\
(\%)\end{array}$} & \multirow[b]{2}{*}{$\begin{array}{l}\text { Input } \\
\text { Value }\end{array}$} & \multicolumn{2}{|c|}{ Method 1} & \multicolumn{2}{|c|}{ Method 2} & \multicolumn{2}{|c|}{ Method 3} \\
\hline & & $\begin{array}{l}\text { Mean } \\
\text { PNC }\end{array}$ & $\begin{array}{c}\text { Change in } \\
\text { Mean PNC } \\
(\%)\end{array}$ & $\begin{array}{c}\text { Mean } \\
\text { PNC }\end{array}$ & $\begin{array}{c}\text { Change in } \\
\text { Mean PNC } \\
(\%)\end{array}$ & $\begin{array}{c}\text { Mean } \\
\text { PNC }\end{array}$ & $\begin{array}{c}\text { Change in } \\
\text { Mean PNC } \\
(\%)\end{array}$ \\
\hline-30 & 49 & 0.0066 & -88.82 & 0.0056 & -89.25 & 0.0062 & -88.42 \\
\hline-20 & 56 & 0.0172 & -70.81 & 0.0147 & -71.71 & 0.0161 & -70.06 \\
\hline-10 & 63 & 0.0333 & -43.49 & 0.0303 & -41.81 & 0.0326 & -39.26 \\
\hline Base & 70 & 0.0589 & 0.00 & 0.0521 & 0.00 & 0.0536 & 0.00 \\
\hline 10 & 77 & 0.0935 & 58.79 & 0.0870 & 67.14 & 0.0886 & 65.27 \\
\hline 20 & 84 & 0.1419 & 140.92 & 0.1333 & 156.04 & 0.1350 & 151.89 \\
\hline 30 & 91 & 0.2074 & 252.07 & 0.1964 & 277.33 & 0.1966 & 266.68 \\
\hline
\end{tabular}




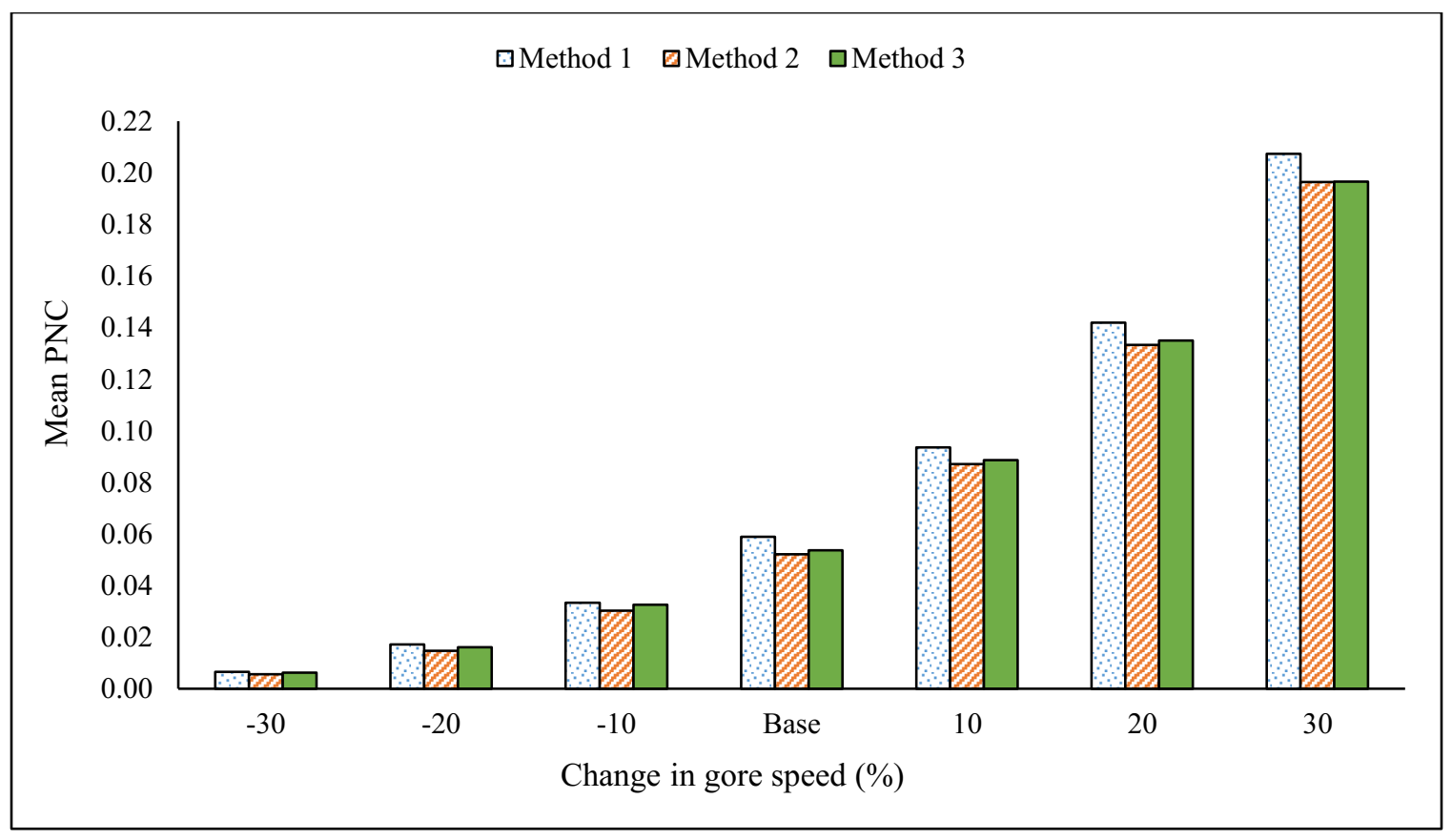

Figure 6.1: Mean PNC vs Change in Gore Speed.

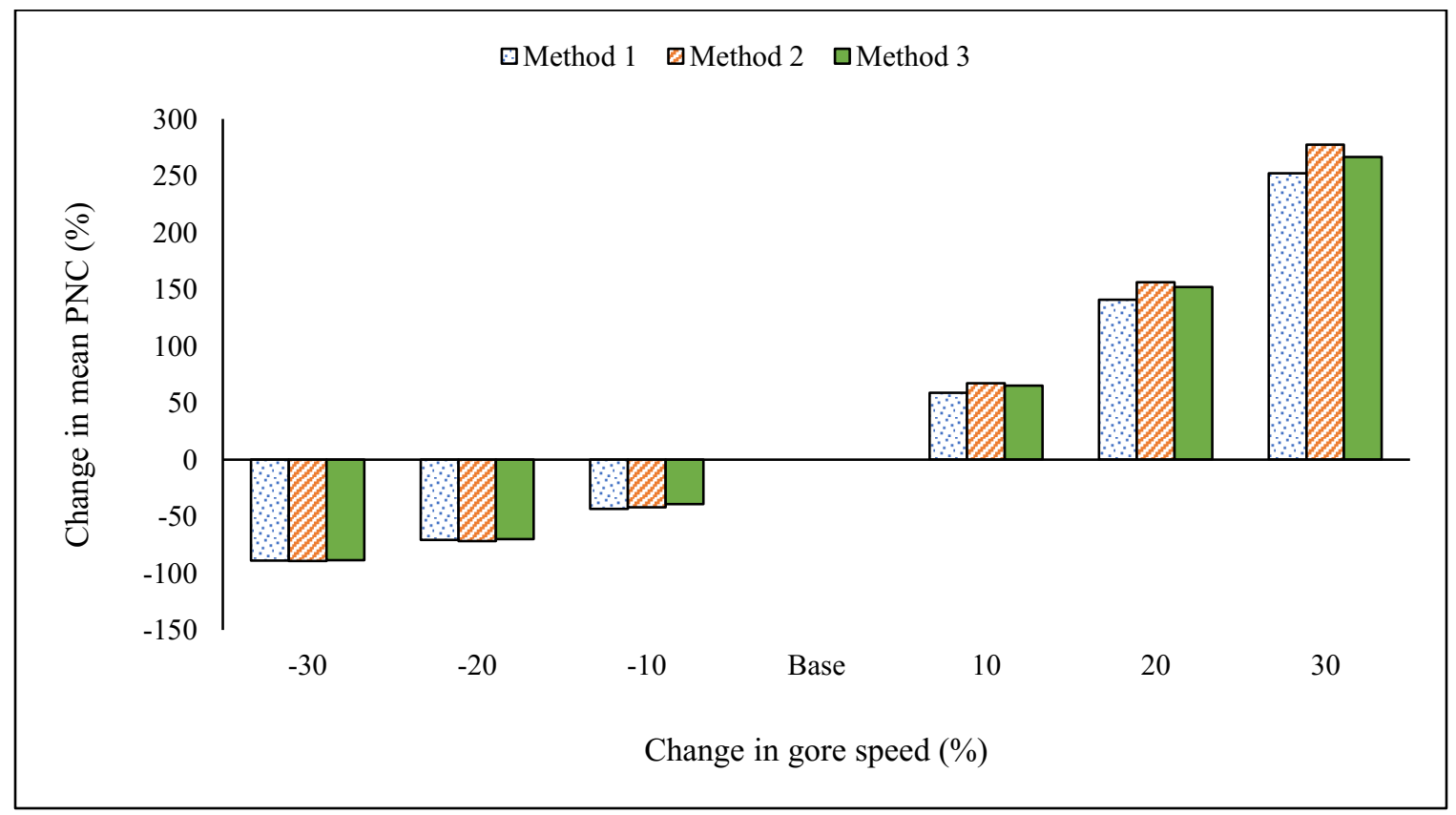

Figure 6.2: Change in Mean PNC vs Change in Gore Speed. 
An additional task was performed to analyse the same effect but with a different base value for the SCL length. The SCL length was changed as $200 \mathrm{~m}$ instead of $400 \mathrm{~m}$ and the results were presented in Table 6.11. It was observed that the mean PNC values increased compared to the longer value of SCL length, but the trend in mean PNC change remained the same. These results also indicate the effect of change in SCL length on mean PNC, which was analysed in Section 6.4.8. For the lower value of SCL length, the percentage changes in mean PNC were smaller than those with the higher value of SCL length.

Table 6.11: Mean PNC and Change in Mean PNC for the Change in Gore Speed.

\begin{tabular}{|c|c|c|c|c|c|c|c|}
\hline \multicolumn{8}{|c|}{ Gore Speed $(\mathrm{km} / \mathrm{h})$} \\
\hline \multirow{2}{*}{$\begin{array}{c}\text { Percentage } \\
\text { change in } \\
\text { parameter } \\
(\%)\end{array}$} & \multirow[b]{2}{*}{$\begin{array}{l}\text { Input } \\
\text { Value }\end{array}$} & \multicolumn{2}{|c|}{ Method 1} & \multicolumn{2}{|c|}{ Method 2} & \multicolumn{2}{|c|}{ Method 3} \\
\hline & & $\begin{array}{c}\text { Mean } \\
\text { PNC }\end{array}$ & $\begin{array}{c}\text { Percentage } \\
\text { change in } \\
\text { mean PNC } \\
(\%)\end{array}$ & $\begin{array}{c}\text { Mean } \\
\text { PNC }\end{array}$ & $\begin{array}{c}\text { Percentage } \\
\text { change in } \\
\text { mean PNC } \\
(\%)\end{array}$ & $\begin{array}{c}\text { Mean } \\
\text { PNC }\end{array}$ & $\begin{array}{c}\text { Percentage } \\
\text { change in } \\
\text { mean PNC } \\
(\%)\end{array}$ \\
\hline-30 & 49 & 0.1448 & -31.98 & 0.1263 & -33.40 & 0.1264 & -31.19 \\
\hline-20 & 56 & 0.1658 & -22.10 & 0.1450 & -23.51 & 0.1427 & -22.34 \\
\hline-10 & 63 & 0.1933 & -9.19 & 0.1646 & -13.21 & 0.1563 & -14.94 \\
\hline Base & 70 & 0.2129 & 0.00 & 0.1896 & 0.00 & 0.1837 & 0.00 \\
\hline 10 & 77 & 0.2464 & 15.78 & 0.2271 & 19.78 & 0.2145 & 16.76 \\
\hline 20 & 84 & 0.2927 & 37.50 & 0.2732 & 44.09 & 0.2677 & 45.71 \\
\hline 30 & 91 & 0.3468 & 62.92 & 0.3310 & 74.58 & 0.3316 & 80.47 \\
\hline
\end{tabular}

\subsubsection{Effect of Change in FRL Speed}

Table 6.12 displays the mean PNC values and percentage changes in the mean PNC values corresponding to the change in FRL speed. Figure 6.3 shows the mean PNC values for the change in FRL speed and Figure 6.4 shows the changes in mean PNC for the change in FRL speed. It was noted that the mean PNC decreased among the different methods. The 
maximum decrease in mean PNC occurred when the FRL speed was reduced by $30 \%$ and the maximum increase in mean PNC occurred when the FRL speed was increased by $30 \%$. However, the percentage change in mean PNC did not show any specific trend of increasing or decreasing with the change in FRL speed. These changes in mean PNC corresponding to the change in FRL speed were considered substantial. As explained in Section 5.2.2.c, headways distribution depends on the hourly traffic volume but not on the speed of the vehicles which means that the gap patterns on FRL will not change according to the speed of the FRL vehicles. If the FRL vehicles are moving at higher speed, the time provided to the SCL drivers to complete the merging action would be less when compared to the vehicles moving at lower speed even though there are adequate gaps available to accept. This could be attributed to the changes in mean PNC with the change in FRL speed.

Table 6.12: Mean PNC and Change in Mean PNC for the Change in FRL Speed.

\begin{tabular}{|c|c|c|c|c|c|c|c|}
\hline \multicolumn{7}{|c|}{ Mean FRL Speed (km/h) } \\
\hline $\begin{array}{c}\text { Change in } \\
\text { Parameter } \\
(\%)\end{array}$ & $\begin{array}{c}\text { Input } \\
\text { Value }\end{array}$ & $\begin{array}{c}\text { Mean } \\
\text { PNC }\end{array}$ & $\begin{array}{c}\text { Change in } \\
\text { Mean } \\
\text { PNC (\%) }\end{array}$ & $\begin{array}{c}\text { Mean } \\
\text { PNC }\end{array}$ & $\begin{array}{c}\text { Change in } \\
\text { Mean } \\
\text { PNC (\%) }\end{array}$ & $\begin{array}{c}\text { Mean } \\
\text { PNC }\end{array}$ & $\begin{array}{c}\text { Change in } \\
\text { Mean } \\
\text { PNC (\%) }\end{array}$ \\
\hline-30 & 71.51 & 0.0399 & -33.01 & 0.0386 & -28.25 & 0.0373 & -31.15 \\
\hline-20 & 81.73 & 0.0538 & -9.57 & 0.0470 & -12.56 & 0.0461 & -14.83 \\
\hline-10 & 91.94 & 0.0591 & -0.71 & 0.0533 & -0.90 & 0.0569 & 4.94 \\
\hline Base & 102.16 & 0.0595 & 0.00 & 0.0537 & 0.00 & 0.0542 & 0.00 \\
\hline 10 & 112.38 & 0.0582 & -2.19 & 0.0530 & -1.31 & 0.0548 & 1.06 \\
\hline 20 & 122.59 & 0.0590 & -0.91 & 0.0542 & 0.77 & 0.0556 & 2.58 \\
\hline 30 & 132.81 & 0.0639 & 7.30 & 0.0564 & 4.95 & 0.0591 & 9.01 \\
\hline
\end{tabular}




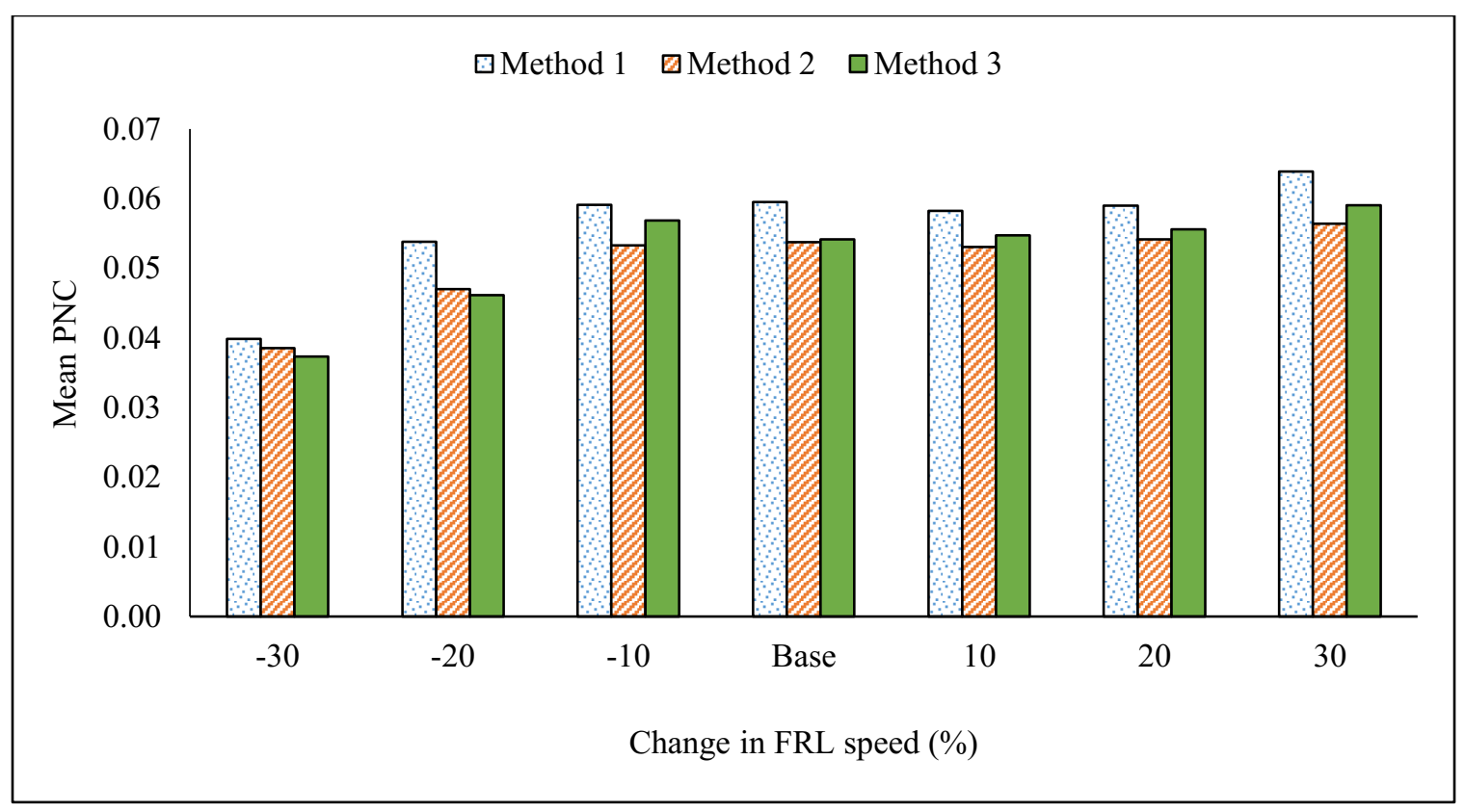

Figure 6.3: Mean PNC vs Change in FRL Speed.

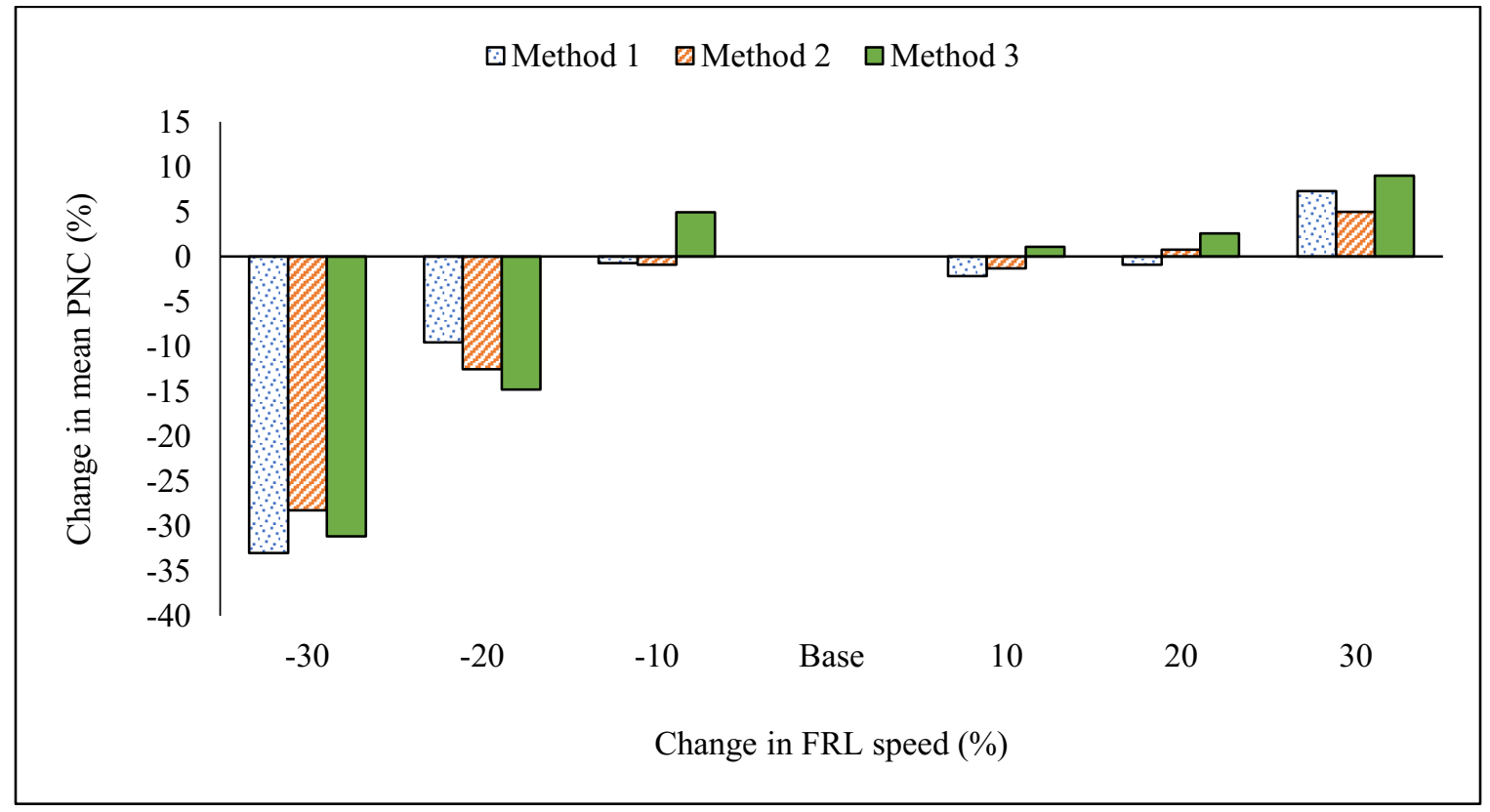

Figure 6.4: Change in Mean PNC vs Change in FRL Speed. 


\subsubsection{Effect of Change in F2L Speed}

Table 6.13 displays the mean PNC values and percentage changes in the mean PNC values corresponding to the change in F2L speed. Figure 6.5 shows the mean PNC values for the change in F2L speed and Figure 6.6 shows the changes in mean PNC for the change in F2L speed. It should be noted that the F2L was not decreased from the base value and only considered the increase from the base value up to $60 \%$. This is to ensure that the F2L speed is always equal to or higher than the FRL speed. It was observed from Table 6.13 that the change in mean PNC is relatively very little and did not show any trend of increase or decrease. These changes could be attributed to the randomization effects of the different input parameters that were randomly generated in the simulation and the same effect was already addressed when deciding the optimum number of model runs in Section 6.1.

Table 6.13: Mean PNC and Change in Mean PNC for the Change in F2L Speed.

\begin{tabular}{|c|c|c|c|c|c|c|c|}
\hline \multicolumn{2}{|c|}{} & \multicolumn{2}{|c|}{ Method 1 } & \multicolumn{2}{c|}{ Method 2 } & \multicolumn{2}{c|}{ Method 3 } \\
\cline { 4 - 8 } $\begin{array}{c}\text { Change in } \\
\text { Parameter } \\
(\%)\end{array}$ & \multirow{2}{*}{$\begin{array}{c}\text { Input } \\
\text { Value }\end{array}$} & $\begin{array}{c}\text { Mean } \\
\text { PNC }\end{array}$ & $\begin{array}{c}\text { Change in } \\
\text { Mean PNC } \\
(\%)\end{array}$ & $\begin{array}{c}\text { Mean } \\
\text { PNC }\end{array}$ & $\begin{array}{c}\text { Change in } \\
\text { Mean PNC } \\
(\%)\end{array}$ & $\begin{array}{c}\text { Mean } \\
\text { PNC }\end{array}$ & $\begin{array}{c}\text { Change in } \\
\text { Mean PNC } \\
(\%)\end{array}$ \\
\hline Base & 102.16 & 0.0578 & 0.00 & 0.0515 & 0.00 & 0.0545 & 0.00 \\
\hline 10 & 112.38 & 0.0575 & -0.57 & 0.0534 & 3.65 & 0.0554 & 1.56 \\
\hline 20 & 122.59 & 0.0579 & 0.12 & 0.0522 & 1.37 & 0.0559 & 2.52 \\
\hline 30 & 132.81 & 0.0571 & -1.18 & 0.0533 & 3.54 & 0.0556 & 2.02 \\
\hline 40 & 143.02 & 0.0575 & -0.58 & 0.0543 & 5.33 & 0.0566 & 3.72 \\
\hline 50 & 153.24 & 0.0552 & -4.54 & 0.0539 & 4.69 & 0.0559 & 2.44 \\
\hline 60 & 163.46 & 0.0574 & -0.67 & 0.0542 & 5.24 & 0.0527 & -3.26 \\
\hline
\end{tabular}




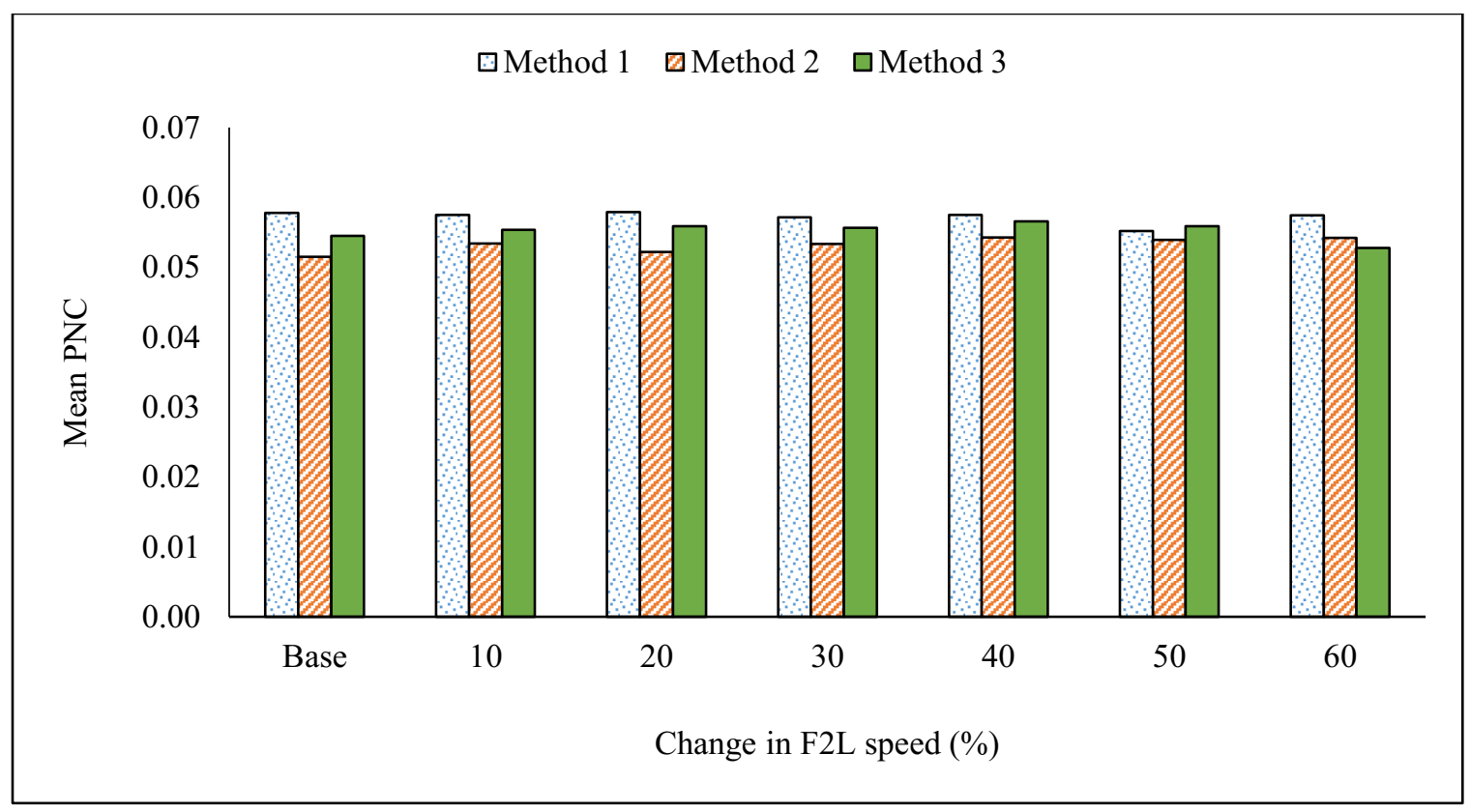

Figure 6.5: Mean PNC vs Change in F2L Speed.

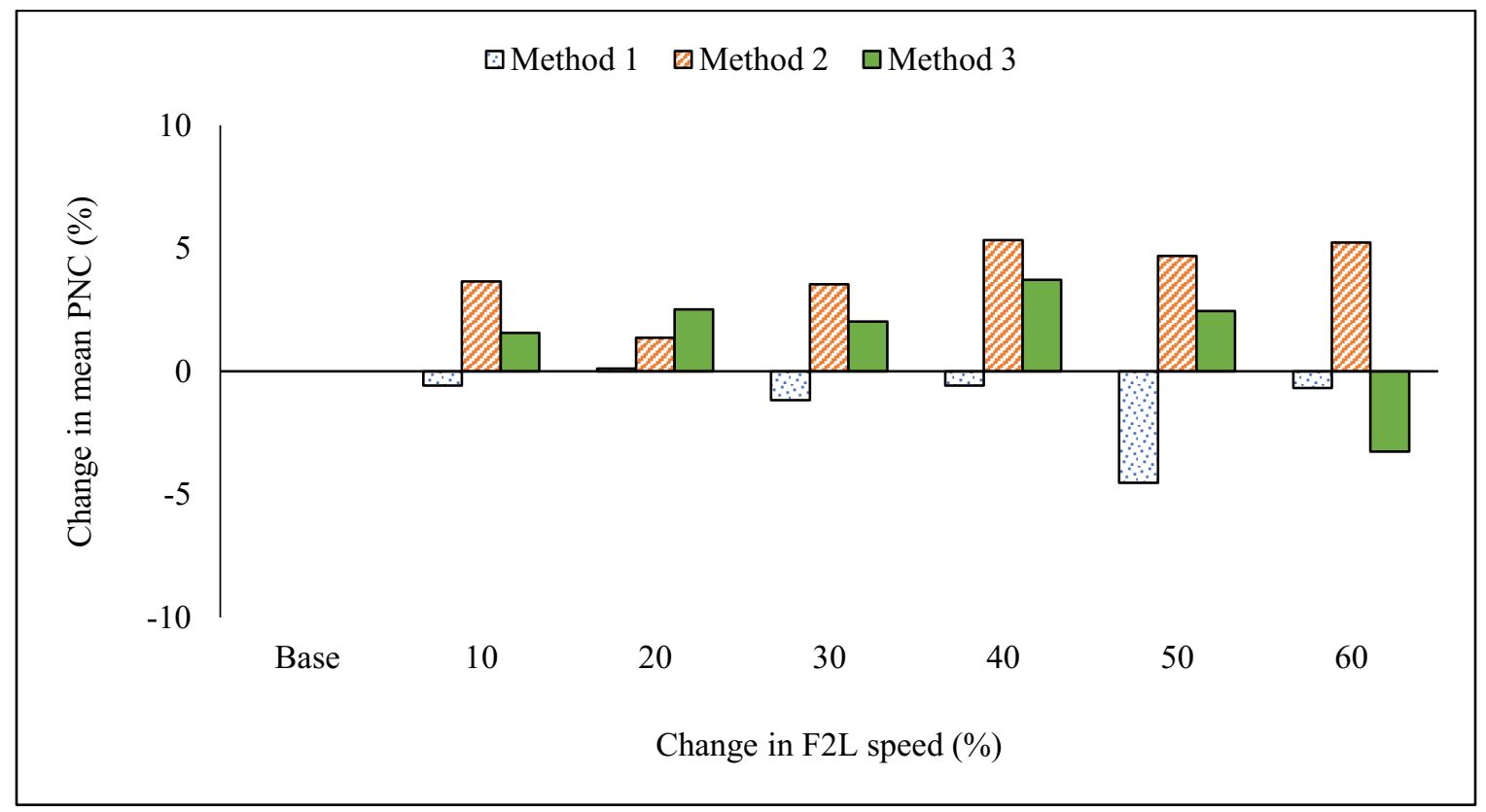

Figure 6.6: Change in Mean PNC vs Change in F2L Speed. 


\subsubsection{Effect of Change in Percentage of HV at FRL}

Percentages of HV on FRL considered for sensitivity analysis are 10\%, 20\%, 30\% and 40\%. Table 6.14 displays the mean PNC values and percentage changes in the mean PNC values corresponding to each percentage of HV on FRL. Figure 6.7 shows the mean PNC values for the change in percentage of HV on FRL and Figure 6.8 shows the changes in mean PNC for the change in percentage of HV on FRL. It was observed from Table 6.14 that the change in mean PNC is relatively much less and the mean PNC increased with the increase in the percentage of $\mathrm{HV}$, except in one scenario. Even though, the percentage changes in mean PNC in some scenarios were increasing, the mean PNC values were very low. The highest change was observed when the percentage of HV on FRL is $40 \%$, which is not a most common situation in the real world. Also, these percentage changes in mean PNC were comparatively low when compared to the percentage changes in mean PNC with the changes in other parameters like gore speed or FRL volume or SCL length.

Table 6.14: Mean PNC and Change in Mean PNC with Change in Percentage of HV on FRL.

\begin{tabular}{|c|c|c|c|c|c|c|}
\hline \multicolumn{7}{|c|}{ Percentage of HV on FRL (\%) } \\
\hline \multirow{2}{*}{$\begin{array}{c}\text { Input } \\
\text { Value }\end{array}$} & \multicolumn{2}{|c|}{ Method 1 } & \multicolumn{2}{c|}{ Method 2 } & \multicolumn{2}{c|}{ Method 3 } \\
\cline { 2 - 7 } & $\begin{array}{c}\text { Mean } \\
\text { PNC }\end{array}$ & $\begin{array}{c}\text { Percentage } \\
\text { change in } \\
\text { mean PNC } \\
\mathbf{( \% )}\end{array}$ & $\begin{array}{c}\text { Mean } \\
\text { PNC }\end{array}$ & $\begin{array}{c}\text { Percentage } \\
\text { change in } \\
\text { mean PNC } \\
\mathbf{( \% )}\end{array}$ & $\begin{array}{c}\text { Mean } \\
\text { PNC }\end{array}$ & $\begin{array}{c}\text { Percentage } \\
\text { change in } \\
\text { mean PNC } \\
\mathbf{( \% )}\end{array}$ \\
\hline 0.10 & 0.0580 & 0.00 & 0.0537 & 0.00 & 0.0535 & 0.00 \\
\hline 0.20 & 0.0585 & 1.00 & 0.0536 & -0.17 & 0.0582 & 8.77 \\
\hline 0.30 & 0.0589 & 1.57 & 0.0555 & 3.31 & 0.0593 & 10.75 \\
\hline 0.40 & 0.0639 & 10.25 & 0.0574 & 6.87 & 0.0597 & 11.61 \\
\hline
\end{tabular}




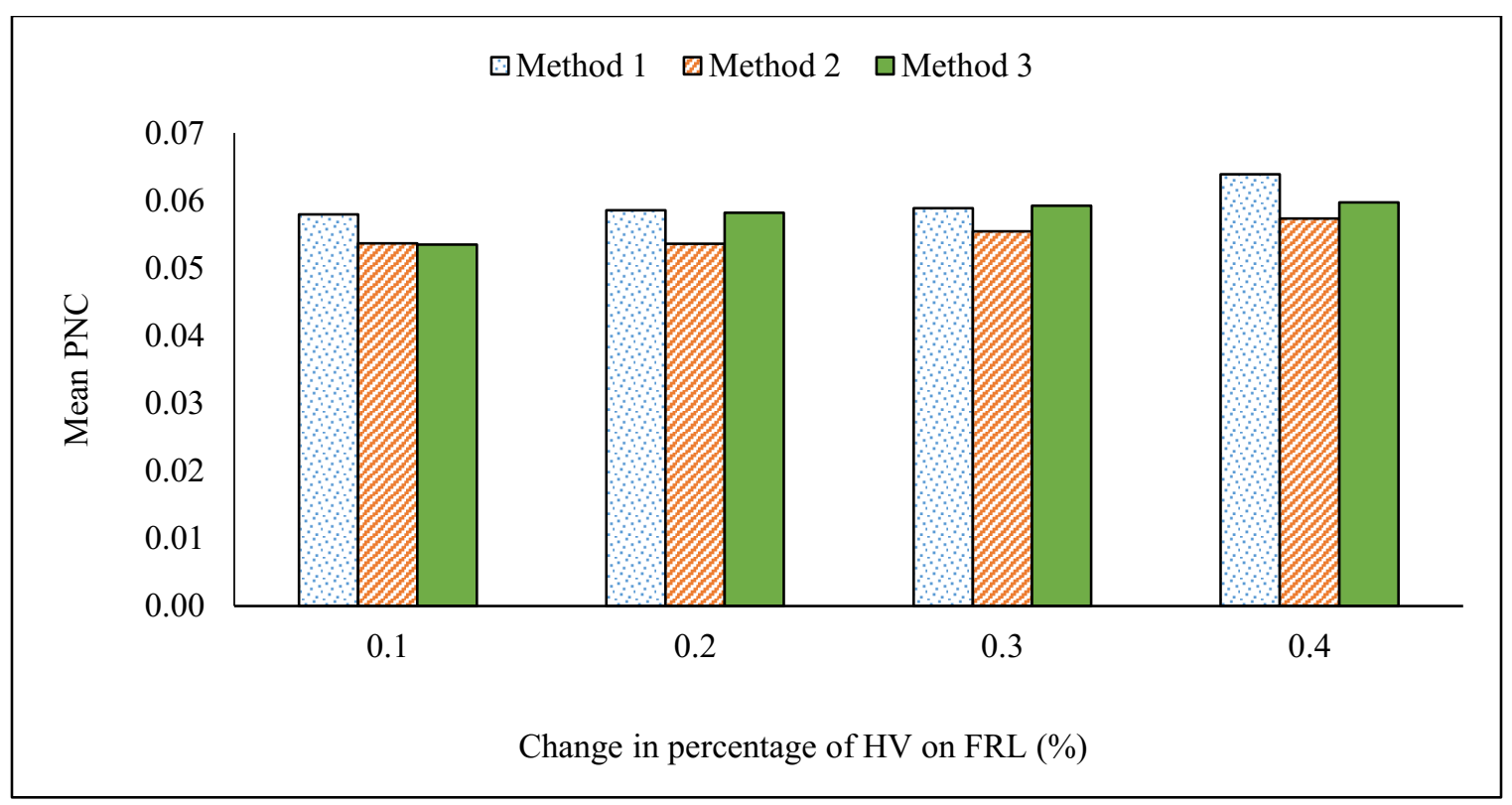

Figure 6.7: Mean PNC vs Change in Percentage of HV on FRL.

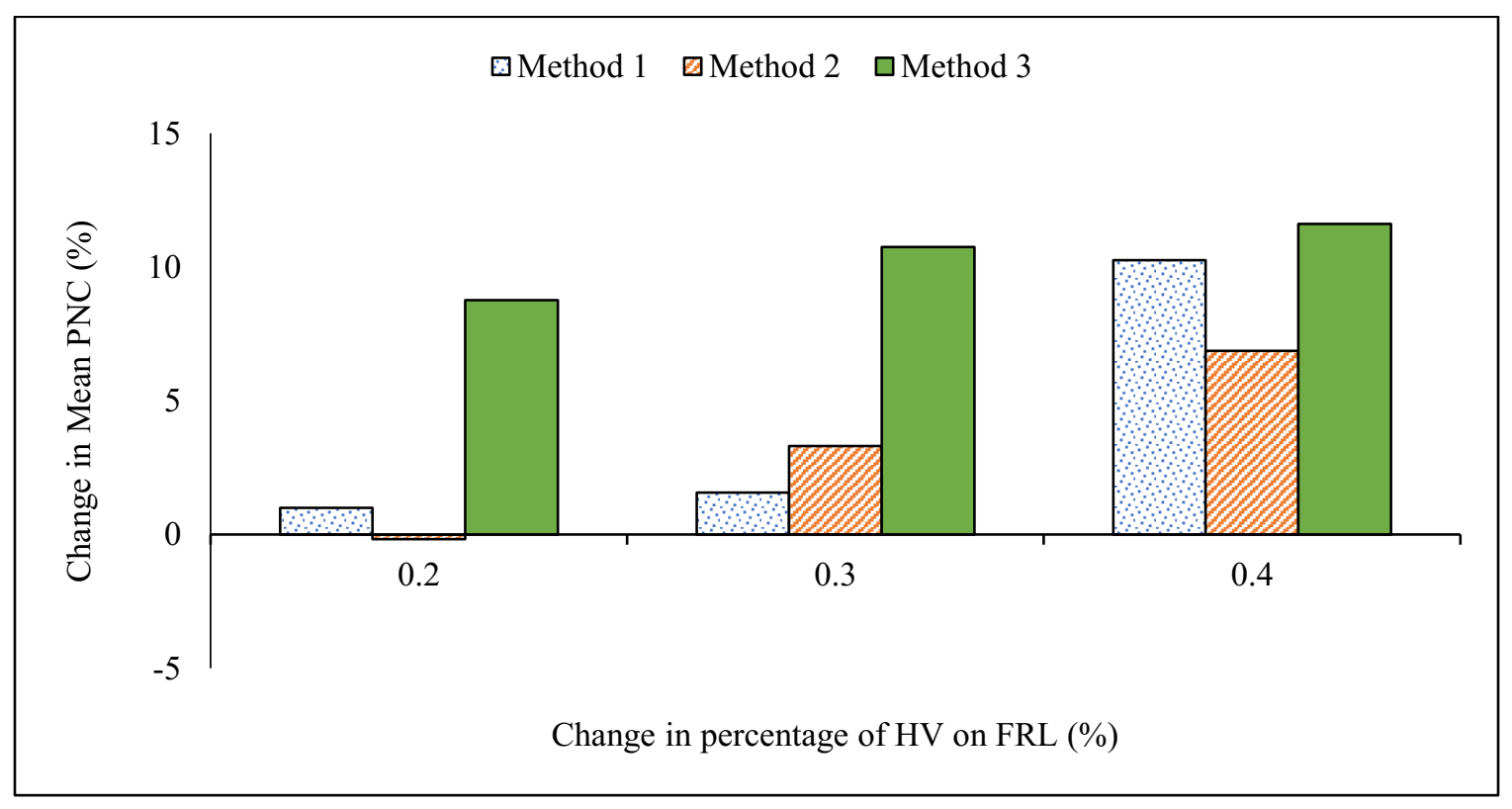

Figure 6.8: Change in Mean PNC vs Change in Percentage of HV on FRL. 


\subsubsection{Effect of Change in Percentage of $\mathrm{HV}$ at F2L}

Percentages of $\mathrm{HV}$ on F2L considered for sensitivity analysis are 10\%, 20\%, 30\% and $40 \%$. Table 6.15 displays the mean PNC values and percentage changes in the mean PNC values corresponding to the change in percentage of $\mathrm{HV}$ on F2L. Figure 6.9 shows the mean PNC values for the change in percentage of $\mathrm{HV}$ on F2L and Figure 6.10 shows the changes in mean PNC for the change in percentage of HV on F2L. It was noticed from Table 6.15 that the change in mean PNC is relatively very little and did not show any trend of increase or decrease. All the percentage changes in mean PNC were less than 5\% and these changes could be attributed to the randomization effects of the different input parameters that were randomly generated in the simulation. The same effect was considered to be accounted for when deciding the optimum number of model runs for all the methods.

Table 6.15: Mean PNC and change in mean PNC with change in percentage of HV on F2L.

\begin{tabular}{|c|c|c|c|c|c|c|}
\hline \multicolumn{7}{|c|}{ Percentage of HV on F2L (\%) } \\
\hline \multirow[b]{2}{*}{$\begin{array}{l}\text { Input } \\
\text { Value }\end{array}$} & \multicolumn{2}{|c|}{ Method 1} & \multicolumn{2}{|c|}{ Method 2} & \multicolumn{2}{|c|}{ Method 3} \\
\hline & $\begin{array}{c}\text { Mean } \\
\text { PNC }\end{array}$ & $\begin{array}{c}\text { Percentage } \\
\text { change in } \\
\text { mean PNC } \\
(\%)\end{array}$ & $\begin{array}{c}\text { Mean } \\
\text { PNC }\end{array}$ & $\begin{array}{c}\text { Percentage } \\
\text { change in } \\
\text { mean PNC } \\
(\%)\end{array}$ & $\begin{array}{l}\text { Mean } \\
\text { PNC }\end{array}$ & $\begin{array}{c}\text { Percentage } \\
\text { change in } \\
\text { mean PNC } \\
(\%)\end{array}$ \\
\hline 0.10 & 0.0581 & 0.00 & 0.0533 & 0.00 & 0.0543 & 0.00 \\
\hline 0.20 & 0.0569 & -2.13 & 0.0534 & 0.15 & 0.0550 & 1.25 \\
\hline 0.30 & 0.0570 & -1.85 & 0.0518 & -2.91 & 0.0551 & 1.38 \\
\hline 0.40 & 0.0595 & 2.42 & 0.0534 & 0.20 & 0.0541 & -0.46 \\
\hline
\end{tabular}




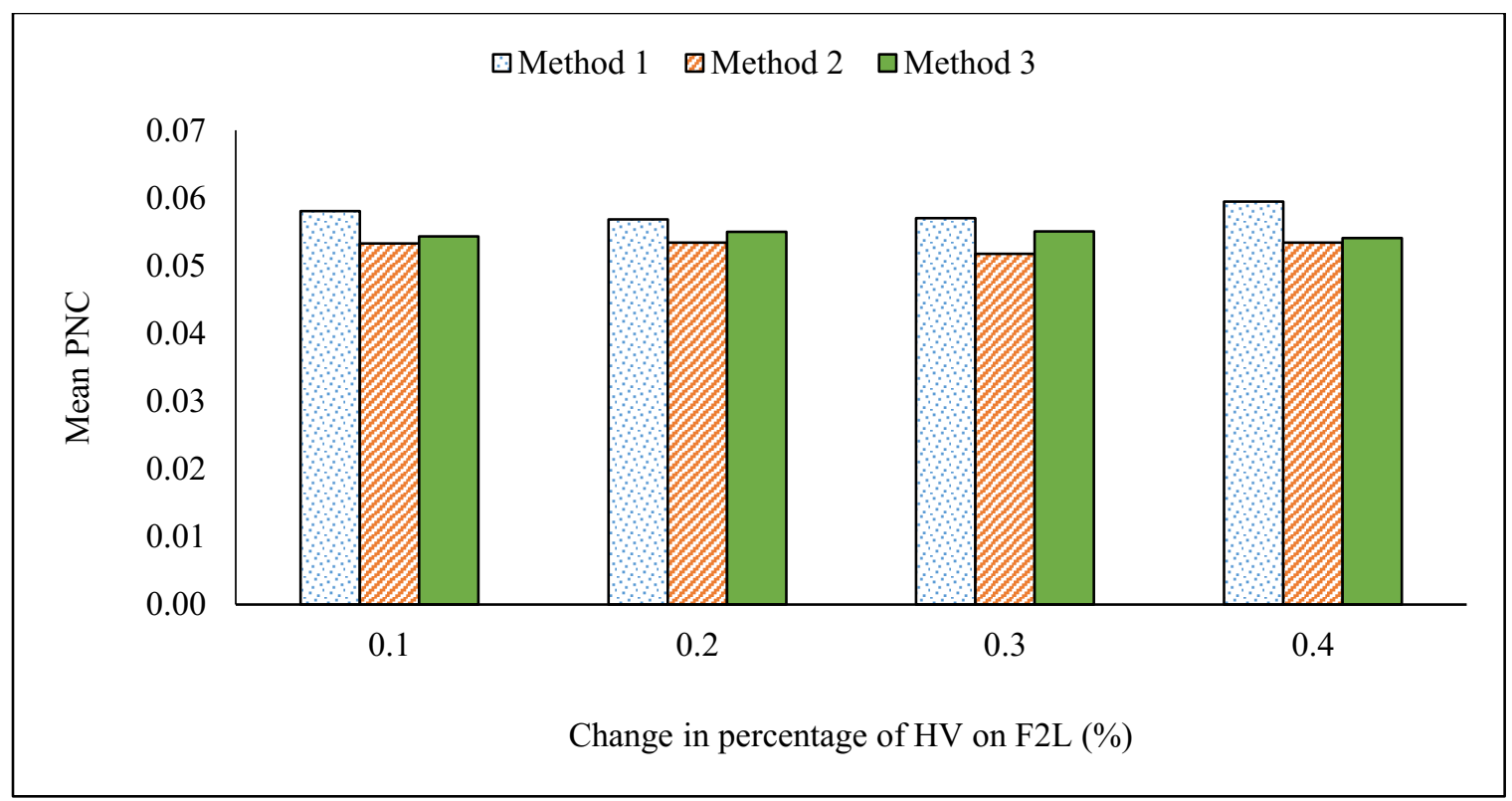

Figure 6.9: Mean PNC vs Change in Percentage of $\mathrm{HV}$ on F2L.

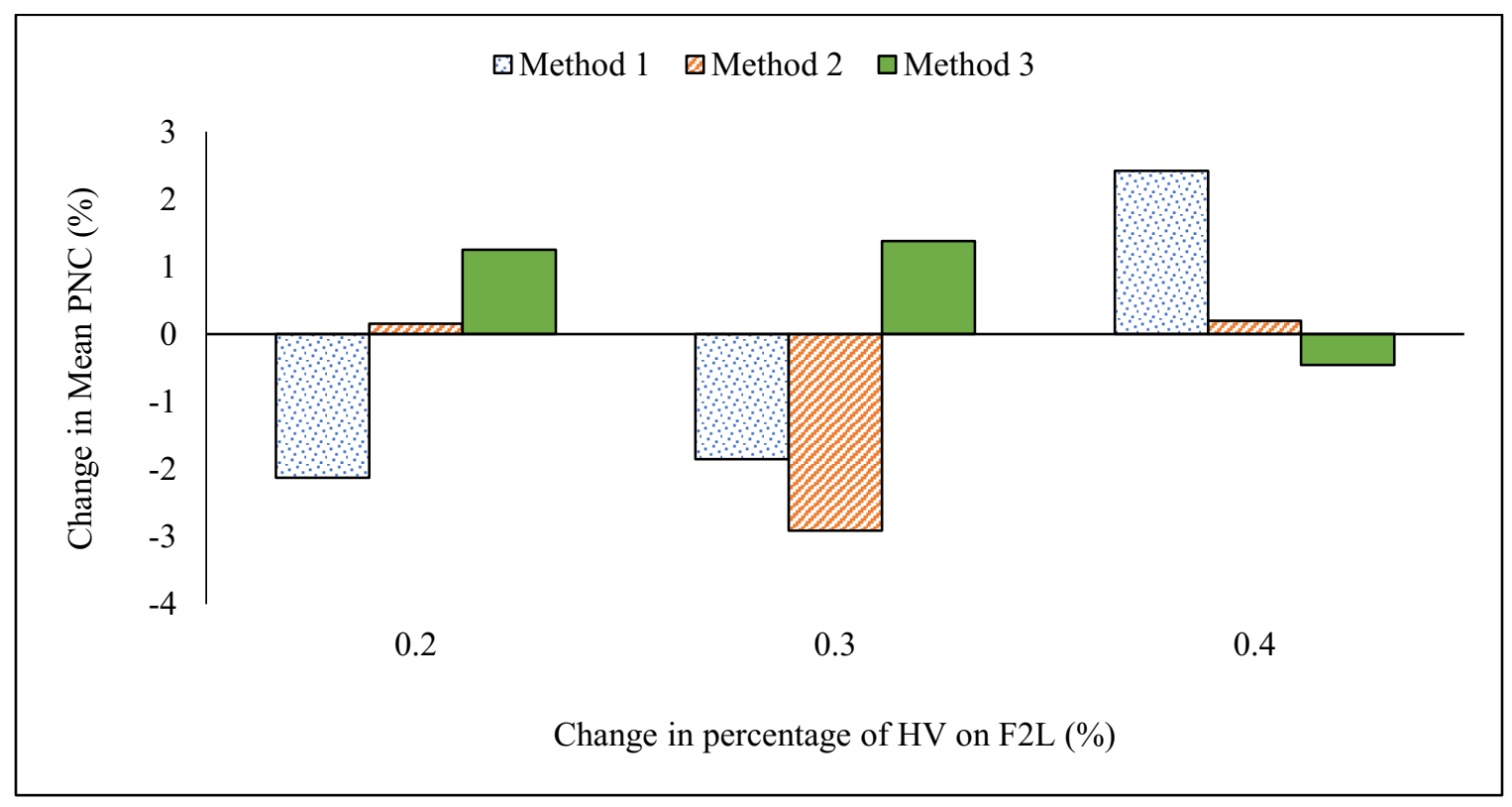

Figure 6.10: Change in Mean PNC vs Change in Percentage of HV on F2L. 


\subsubsection{Effect of Change in FRL Traffic Volume}

Table 6.16 displays the mean PNC values and percentage changes in the mean PNC values corresponding to the change in FRL traffic volume. Figure 6.11 shows the mean PNC values for the change in FRL traffic volume and Figure 6.12 shows the changes in mean PNC for the change in FRL traffic volume. It was noticed from Table 6.16 that the mean PNC was decreased when FRL traffic volume was decreased and increased when the FRL traffic volume was increased. If the FRL traffic volume was high, the available time gaps would decrease and might be not sufficient for performing a safe merging. This could be attributed to increase in the probability of uncomfortable merging of SCL drivers and vice versa in the case of lower traffic volumes.

Table 6.16: Mean PNC and Change in Mean PNC for the Change in FRL Traffic Volume.

\begin{tabular}{|c|c|c|c|c|c|c|c|}
\hline \multicolumn{8}{|c|}{ FRL Traffic Volume (veh/h) } \\
\hline \multirow{2}{*}{$\begin{array}{c}\text { Change in } \\
\text { Parameter } \\
(\%)\end{array}$} & \multirow[b]{2}{*}{$\begin{array}{l}\text { Input } \\
\text { Value }\end{array}$} & \multicolumn{2}{|c|}{ Method 1} & \multicolumn{2}{|c|}{ Method 2} & \multicolumn{2}{|c|}{ Method 3} \\
\hline & & $\begin{array}{c}\text { Mean } \\
\text { PNC }\end{array}$ & $\begin{array}{l}\text { Change } \\
\text { in Mean } \\
\text { PNC }(\%)\end{array}$ & $\begin{array}{c}\text { Mean } \\
\text { PNC }\end{array}$ & $\begin{array}{l}\text { Change } \\
\text { in Mean } \\
\text { PNC }(\%)\end{array}$ & $\begin{array}{l}\text { Mean } \\
\text { PNC }\end{array}$ & $\begin{array}{l}\text { Change } \\
\text { in Mean } \\
\text { PNC }(\%)\end{array}$ \\
\hline-30 & 560 & 0.0389 & -32.62 & 0.0350 & -34.99 & 0.0361 & -35.08 \\
\hline-20 & 640 & 0.0434 & -24.81 & 0.0408 & -24.15 & 0.0417 & -24.98 \\
\hline-10 & 720 & 0.0505 & -12.60 & 0.0459 & -14.67 & 0.0487 & -12.44 \\
\hline Base & 800 & 0.0578 & 0.00 & 0.0538 & 0.00 & 0.0556 & 0.00 \\
\hline 10 & 880 & 0.0656 & 13.63 & 0.0602 & 11.75 & 0.0633 & 13.80 \\
\hline 20 & 960 & 0.0741 & 28.25 & 0.0711 & 32.08 & 0.0717 & 28.99 \\
\hline 30 & 1040 & 0.0884 & 53.01 & 0.0780 & 44.77 & 0.0827 & 48.63 \\
\hline
\end{tabular}




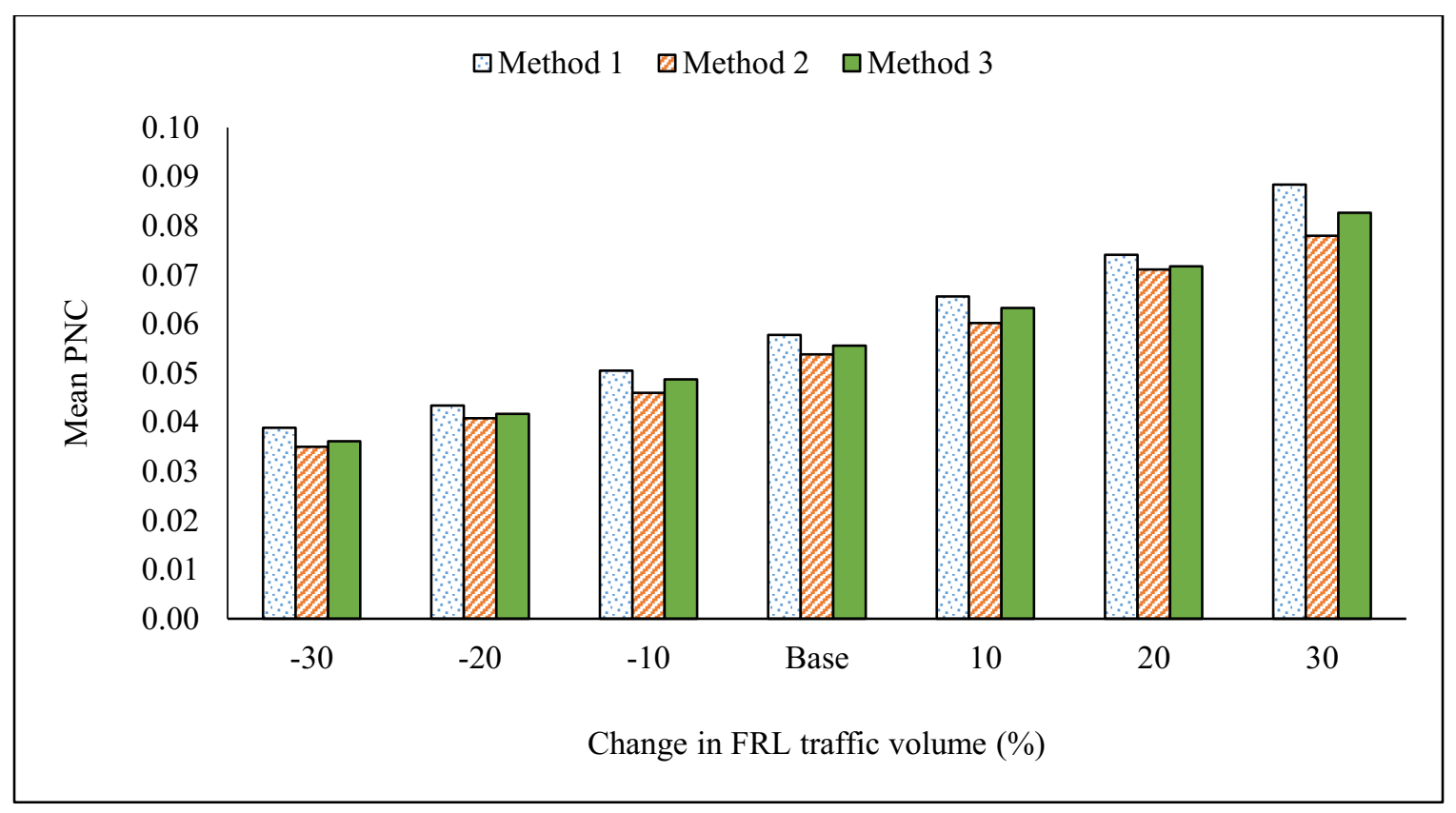

Figure 6.11: Mean PNC vs Change in FRL Traffic Volume.

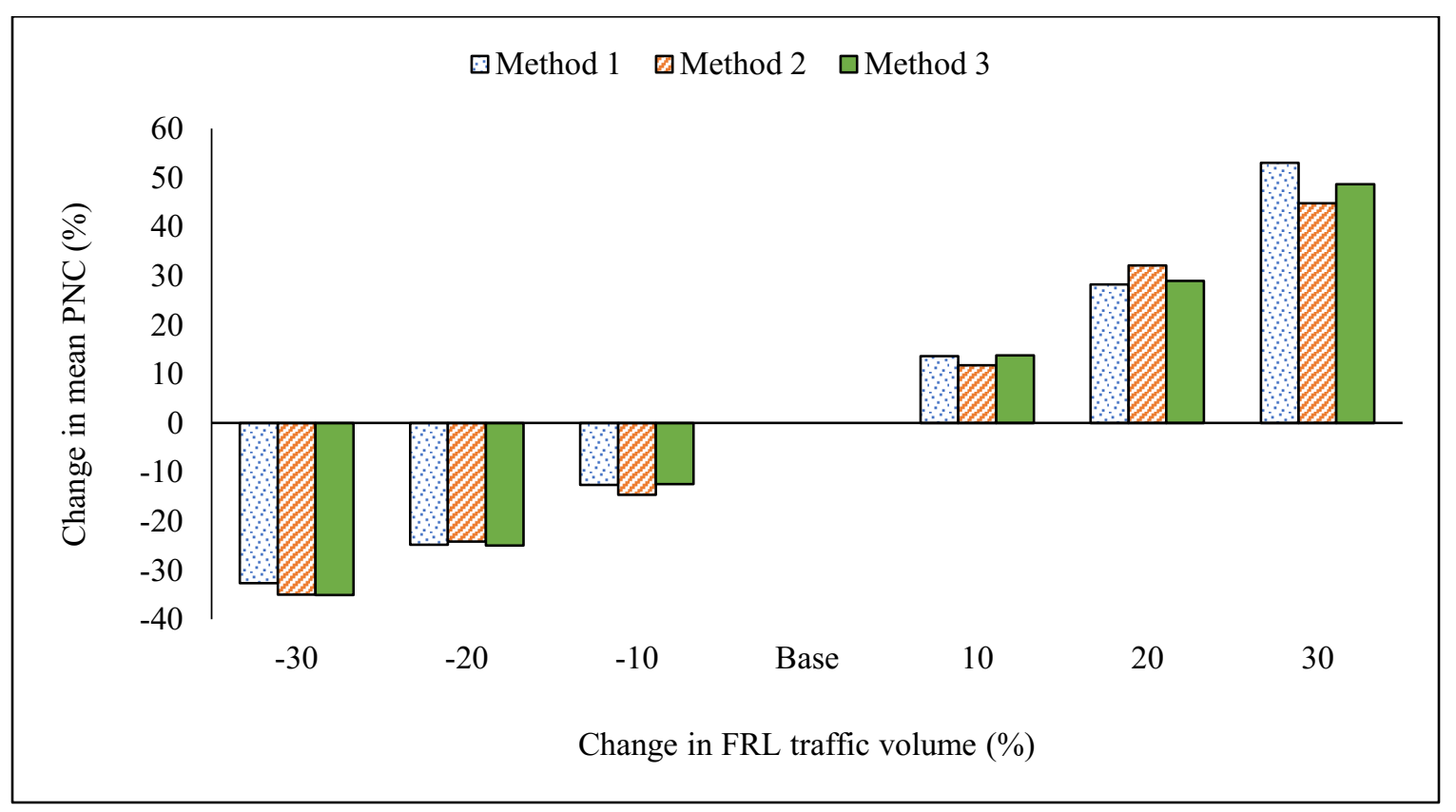

Figure 6.12: Change in Mean PNC vs Change in FRL Traffic Volume. 


\subsubsection{Effect of Change in Lane Ratio}

As mentioned earlier, lane ratio in this study is the ratio between traffic volume on F2L and traffic volume on FRL. Table 6.17 provides the mean PNC values and percentage changes in the mean PNC values corresponding to the change in lane ratio. Figure 6.13 shows the mean PNC values for the change in lane ratio and Figure 6.14 shows the changes in mean PNC for the change in lane ratio. It was noticed from Table 6.17 that the change in mean PNC is relatively very little and did not show any trend of increase or decrease which is similar to the effect of change in percentage of HV on FRL and percentage of HV on F2L. All the percentage changes in mean PNC were less than 5\% and these changes could be attributed to the randomization effects of the different input parameters that were randomly generated in the simulation of SCL vehicles.

Table 6.17: Mean PNC and Change in Mean PNC for the Change in Lane Ratio.

\begin{tabular}{|c|c|c|c|c|c|c|c|}
\hline \multicolumn{8}{|c|}{ Mean Lane Ratio } \\
\hline \multirow{2}{*}{$\begin{array}{c}\text { Change in } \\
\text { Parameter } \\
(\%)\end{array}$} & \multirow[b]{2}{*}{$\begin{array}{l}\text { Mean } \\
\text { Lane } \\
\text { Ratio }\end{array}$} & \multicolumn{2}{|c|}{ Method 1} & \multicolumn{2}{|c|}{ Method 2} & \multicolumn{2}{|c|}{ Method 3} \\
\hline & & $\begin{array}{c}\text { Mean } \\
\text { PNC }\end{array}$ & $\begin{array}{l}\text { Change } \\
\text { in Mean } \\
\text { PNC (\%) }\end{array}$ & $\begin{array}{c}\text { Mean } \\
\text { PNC }\end{array}$ & $\begin{array}{l}\text { Change } \\
\text { in Mean } \\
\text { PNC }(\%)\end{array}$ & $\begin{array}{l}\text { Mean } \\
\text { PNC }\end{array}$ & $\begin{array}{l}\text { Change } \\
\text { in Mean } \\
\text { PNC }(\%)\end{array}$ \\
\hline-30 & 1.05 & 0.0576 & 0.16 & 0.0516 & -3.80 & 0.0543 & -3.25 \\
\hline-20 & 1.20 & 0.0575 & -0.08 & 0.0545 & 1.56 & 0.0539 & -3.86 \\
\hline-10 & 1.35 & 0.0580 & 0.87 & 0.0538 & 0.17 & 0.0535 & -4.64 \\
\hline Base & 1.50 & 0.0575 & 0.00 & 0.0537 & 0.00 & 0.0561 & 0.00 \\
\hline 10 & 1.65 & 0.0575 & 0.05 & 0.0526 & -1.99 & 0.0553 & -1.49 \\
\hline 20 & 1.80 & 0.0572 & -0.61 & 0.0526 & -2.01 & 0.0556 & -0.98 \\
\hline 30 & 1.95 & 0.0577 & 0.34 & 0.0547 & 1.95 & 0.0550 & -2.00 \\
\hline
\end{tabular}




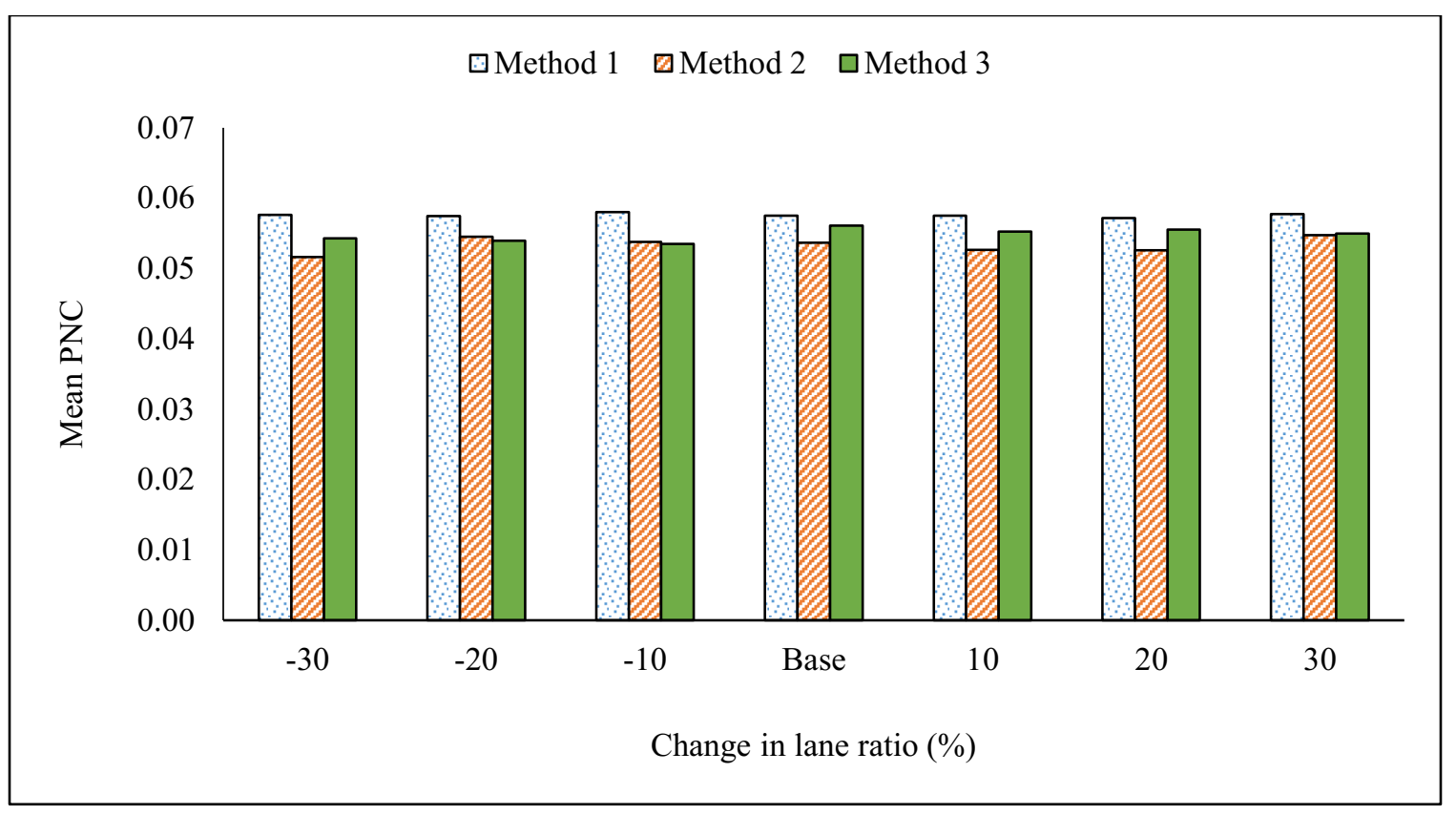

Figure 6.13: Mean PNC vs Change in Lane Ratio.

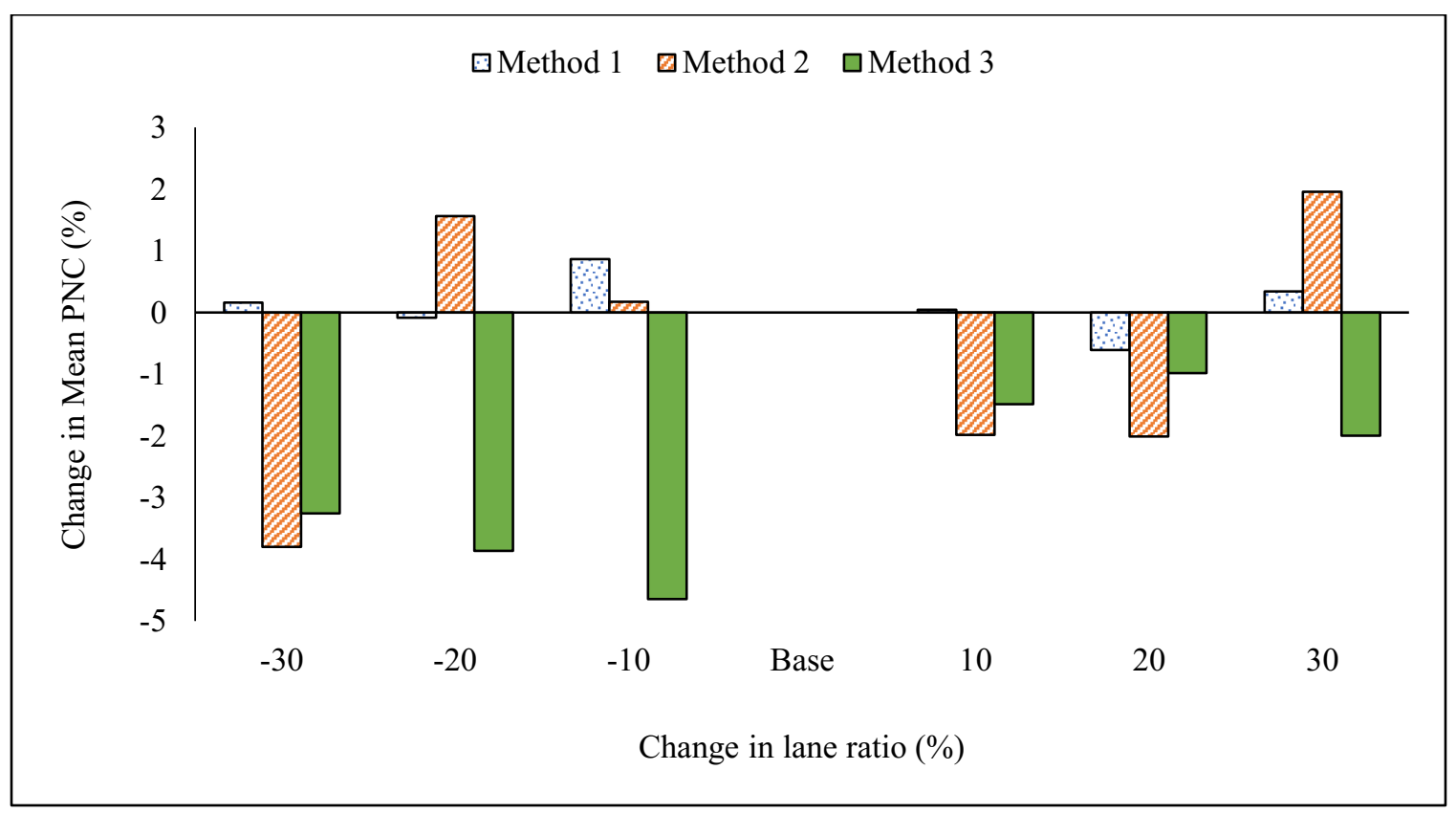

Figure 6.14: Change in Mean PNC vs Change in Lane Ratio. 


\subsubsection{Effect of Change in SCL Length}

Table 6.18 displays the mean PNC values and percentage changes in the mean PNC values corresponding to the change in SCL length. Figure 6.15 shows the mean PNC values for the change in SCL length and Figure 6.16 shows the changes in mean PNC for the change in SCL length. It was noticed from Table 6.18 that the mean PNC was decreased when SCL length was increased and increased when the SCL length was decreased. Longer SCL lengths would provide more time and distance for the SCL drivers for accelerating and gap searching purposes and to merge safely into freeway through traffic. This could be attributed to the decrease in the probability of uncomfortable merging of SCL drivers and vice versa in the case of lower SCL length values.

Table 6.18: Mean PNC and Change in Mean PNC with the Change in SCL Length.

\begin{tabular}{|c|c|c|c|c|c|c|c|}
\hline \multicolumn{8}{|c|}{ SCL Length (m) } \\
\hline \multirow{2}{*}{$\begin{array}{c}\text { Change in } \\
\text { Parameter } \\
(\%)\end{array}$} & \multirow[b]{2}{*}{$\begin{array}{l}\text { Input } \\
\text { Value }\end{array}$} & \multicolumn{2}{|c|}{ Method 1} & \multicolumn{2}{|c|}{ Method 2} & \multicolumn{2}{|c|}{ Method 3} \\
\hline & & $\begin{array}{c}\text { Mean } \\
\text { PNC }\end{array}$ & $\begin{array}{l}\text { Change } \\
\text { in Mean } \\
\text { PNC }(\%)\end{array}$ & $\begin{array}{c}\text { Mean } \\
\text { PNC }\end{array}$ & $\begin{array}{l}\text { Change } \\
\text { in Mean } \\
\text { PNC }(\%)\end{array}$ & $\begin{array}{l}\text { Mean } \\
\text { PNC }\end{array}$ & $\begin{array}{l}\text { Change } \\
\text { in Mean } \\
\text { PNC }(\%)\end{array}$ \\
\hline-30 & 280 & 0.1163 & 95.46 & 0.0988 & 79.81 & 0.0983 & 81.05 \\
\hline-20 & 320 & 0.0904 & 51.93 & 0.0811 & 47.69 & 0.0794 & 46.27 \\
\hline-10 & 360 & 0.0711 & 19.60 & 0.0627 & 14.11 & 0.0647 & 19.17 \\
\hline Base & 400 & 0.0595 & 0.00 & 0.0549 & 0.00 & 0.0543 & 0.00 \\
\hline 10 & 440 & 0.0495 & -16.76 & 0.0450 & -18.02 & 0.0464 & -14.54 \\
\hline 20 & 480 & 0.0414 & -30.37 & 0.0388 & -29.30 & 0.0416 & -23.29 \\
\hline 30 & 520 & 0.0376 & -36.78 & 0.0355 & -35.30 & 0.0368 & -32.26 \\
\hline
\end{tabular}




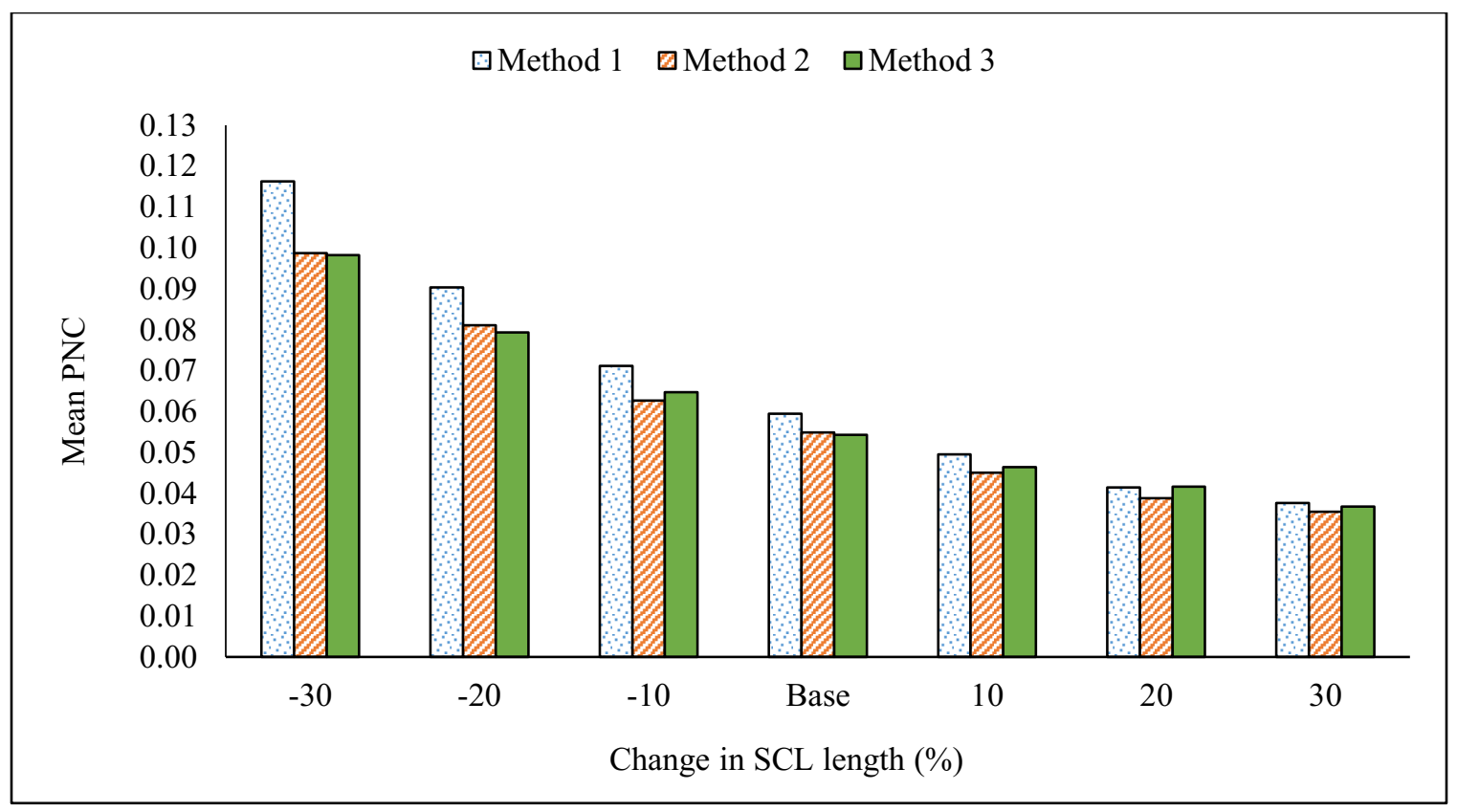

Figure 6.15: Mean PNC vs Change in SCL Length.

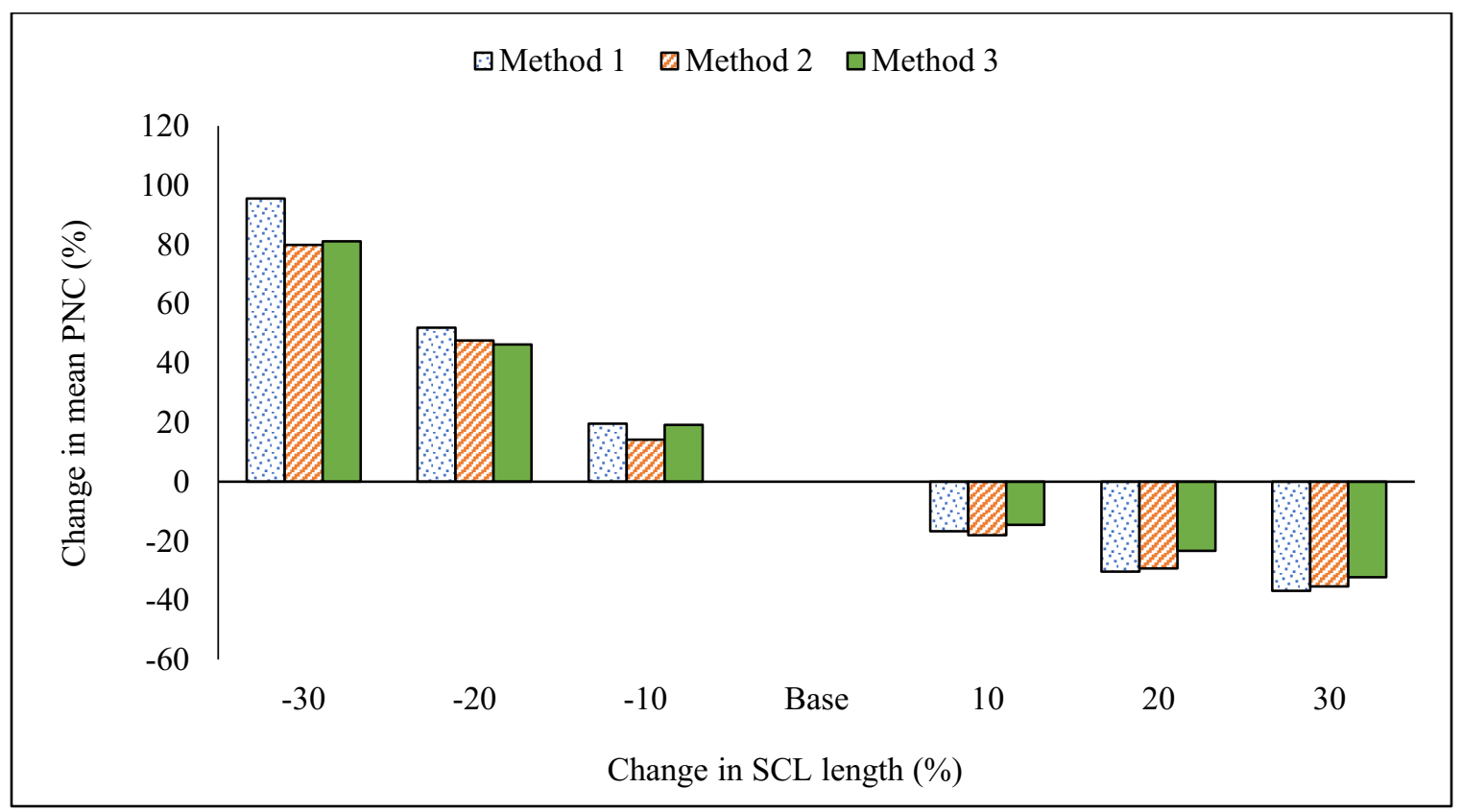

Figure 6.16: Change in Mean PNC vs Change in SCL Length. 


\subsubsection{Effect of Change in SCL Traffic Volume}

Table 6.19 displays the mean PNC values and percentage changes in the mean PNC values corresponding to the change in traffic volume on SCL. Figure 6.17 shows the mean PNC values for the change in SCL traffic volume and Figure 6.18 shows the changes in mean PNC for the change in SCL traffic volume. It was noticed from the Table 6.19 that the percentage change in mean PNC is relatively very less and did not show any trend of increasing or decreasing. All the percentage changes in mean PNC were less than 5\% and these changes could be attributed to the randomization effects of the different input parameters that were randomly generated in the simulation. The same effect had been already accounted for when deciding the optimum number of model runs for all the methods.

Table 6.19: Mean PNC and Change in Mean PNC with the Change in SCL Traffic Volume.

\begin{tabular}{|c|c|c|c|c|c|c|c|}
\hline \multicolumn{8}{|c|}{ SCL Traffic Volume (veh/h) } \\
\hline \multirow{2}{*}{$\begin{array}{c}\text { Change in } \\
\text { Parameter } \\
(\%)\end{array}$} & \multirow[b]{2}{*}{$\begin{array}{l}\text { Input } \\
\text { Value }\end{array}$} & \multicolumn{2}{|c|}{ Method 1} & \multicolumn{2}{|c|}{ Method 2} & \multicolumn{2}{|c|}{ Method 3} \\
\hline & & $\begin{array}{l}\text { Mean } \\
\text { PNC }\end{array}$ & $\begin{array}{l}\text { Change } \\
\text { in Mean } \\
\text { PNC (\%) }\end{array}$ & $\begin{array}{c}\text { Mean } \\
\text { PNC }\end{array}$ & $\begin{array}{l}\text { Change } \\
\text { in Mean } \\
\text { PNC (\%) }\end{array}$ & $\begin{array}{c}\text { Mean } \\
\text { PNC }\end{array}$ & $\begin{array}{l}\text { Change } \\
\text { in Mean } \\
\text { PNC (\%) }\end{array}$ \\
\hline-30 & 280 & 0.0590 & -0.50 & 0.0542 & 0.67 & 0.0556 & 0.27 \\
\hline-20 & 320 & 0.0591 & -0.37 & 0.0564 & 4.76 & 0.0560 & 1.09 \\
\hline-10 & 360 & 0.0579 & -2.34 & 0.0528 & -2.03 & 0.0555 & 0.12 \\
\hline Base & 400 & 0.0593 & 0.00 & 0.0539 & 0.00 & 0.0554 & 0.00 \\
\hline 10 & 440 & 0.0579 & -2.40 & 0.0536 & -0.44 & 0.0554 & 0.03 \\
\hline 20 & 480 & 0.0586 & -1.17 & 0.0536 & -0.46 & 0.0551 & -0.49 \\
\hline 30 & 520 & 0.0574 & -3.18 & 0.0547 & 1.47 & 0.0571 & 3.02 \\
\hline
\end{tabular}




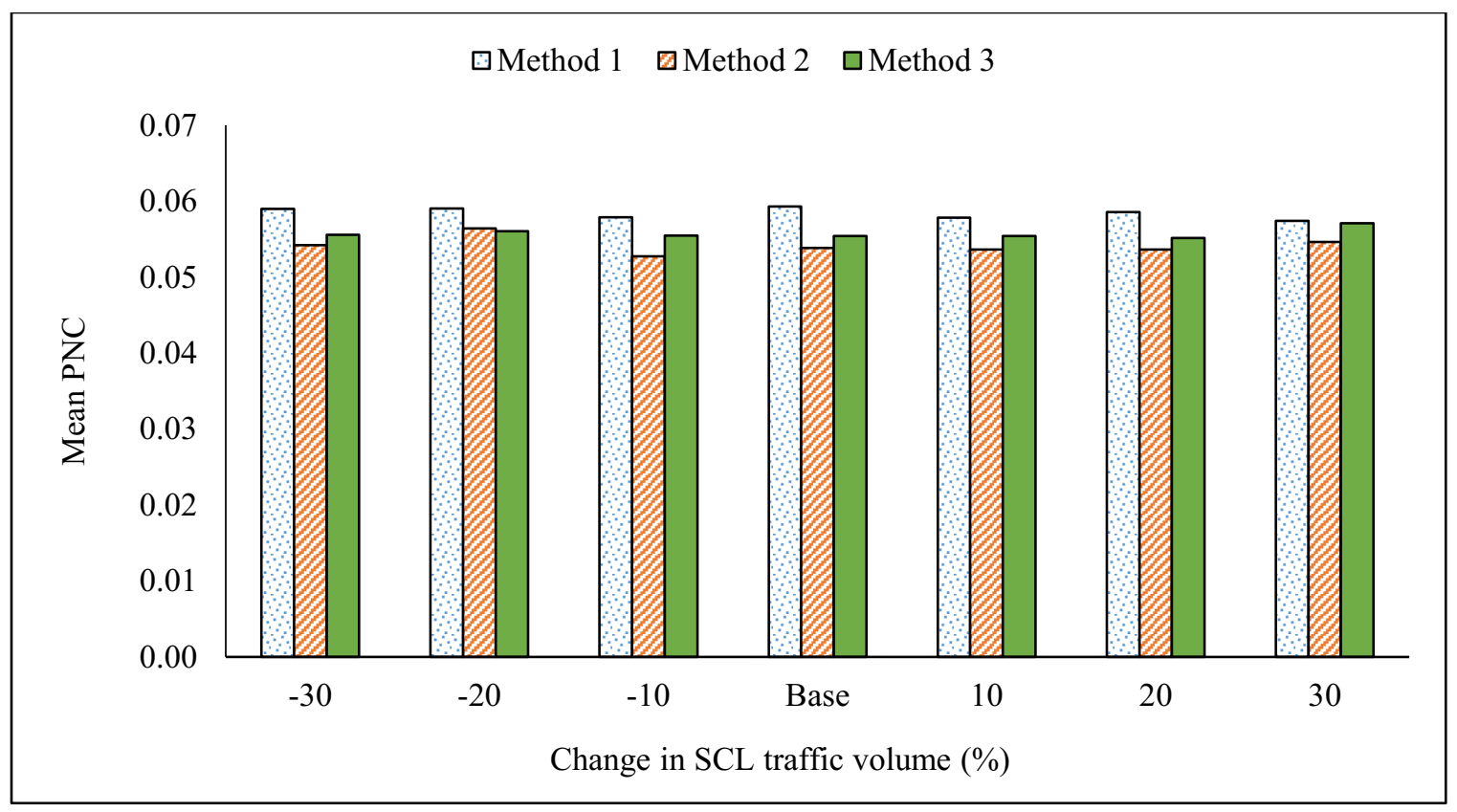

Figure 6.17: Mean PNC vs Change in SCL Traffic Volume.

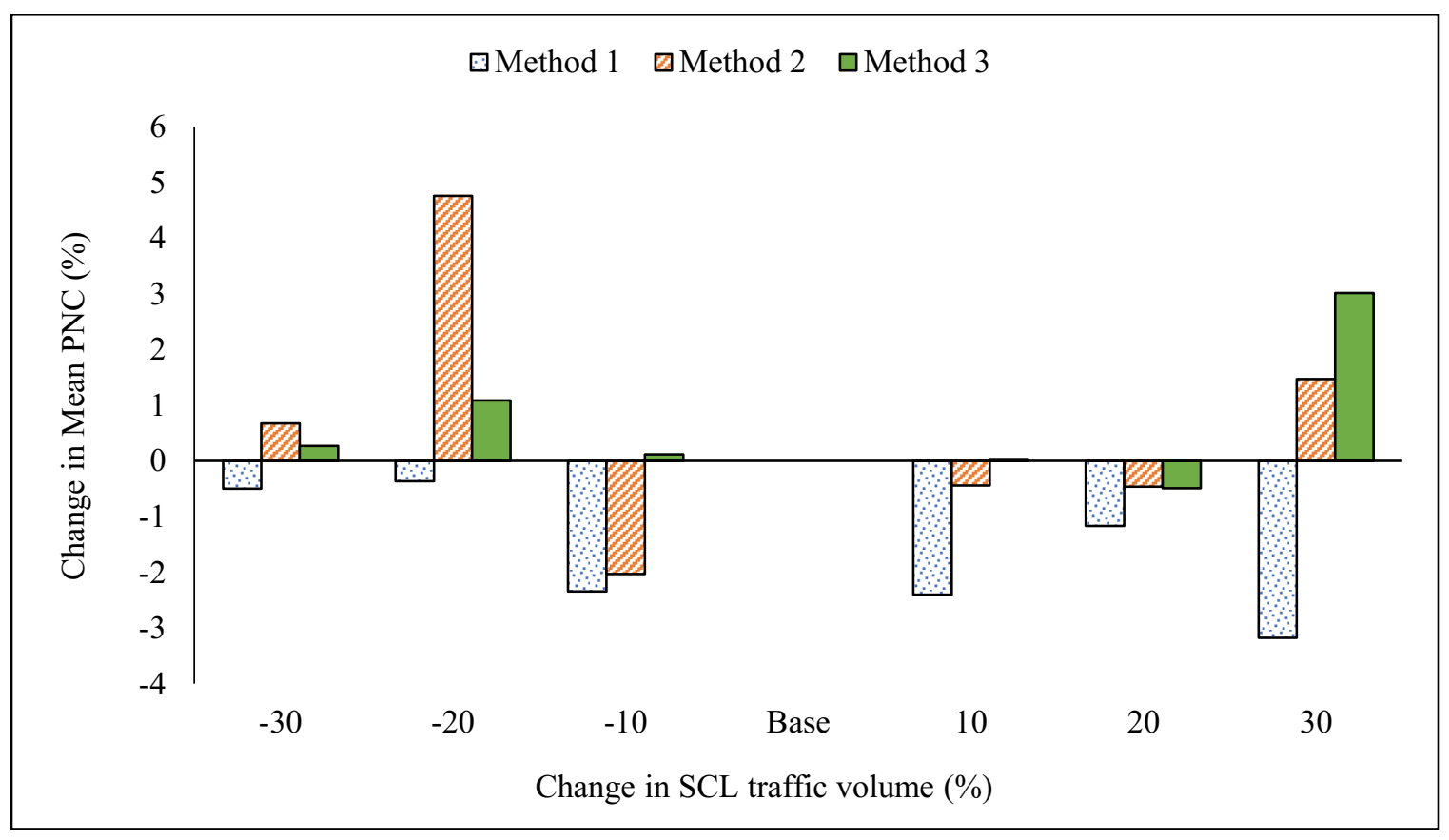

Figure 6.18: Change in Mean PNC vs Change in SCL Traffic Volume. 
From the sensitivity analysis of various parameters, it was observed that the mean PNC was sensitive to the parameters gore speed, FRL speed, FRL volume and SCL length. The other input parameters F2L speed, percentage of HV on FRL and F2L, traffic volume on F2L and SCL did not show any considerable effect on the mean PNC of SCL vehicles.

\subsection{Application of Proposed Methodologies to the Design Guides}

An additional task was performed to examine the changes in mean PNC values for the SCL length values recommended by the design guides AASHTO (2011) and TAC (2017) corresponding to the specific ramp controlling speeds. The input parameters which exhibited sensitivity for mean PNC were considered as variables in this task as shown in and Table 6.21. The other input parameters were assumed based on the average values of each parameter from the field data available because their effect on the mean PNC was considerably low. Assumed values for other input parameters are listed below:

- According to MTO, it is generally a desirable practice to choose a design speed which is $20 \mathrm{~km} / \mathrm{h}$ more than the posted highway speed (Geometric Design Standards for Ontario Highways, 1994). Therefore, design speed of the freeway was chosen as $120 \mathrm{~km} / \mathrm{h}$ for this task.

- Three different values of FRL speed were used for evaluating the PNC: 90, 100, $100 \mathrm{~km} / \mathrm{h}$.

- A constant value of $102.16 \mathrm{~km} / \mathrm{h}$ was assumed for F2L speed which was based on the available field data.

- Three different traffic volumes were taken into consideration: $400,800,1200 \mathrm{veh} / \mathrm{h}$. 
- From the sensitivity analysis, there was no relation found between the percentage of HV on FRL, F2L and the mean PNC. Therefore, the percentage of HV on FRL and F2L was taken as $10 \%$ based on the available field data.

- Since there was no relation found between the lane ratio and the mean PNC, the input value for lane ratio was taken as 1.5 based on the available data of F2L traffic volume.

- Traffic volume on the SCL was chosen as $400 \mathrm{veh} / \mathrm{h}$ based on the data available at 16 SCL sites.

- Three ramp controlling curve speeds and their corresponding recommended SCL lengths were chosen from both AASHTO (2011) and TAC (2017).

Table 6.20 and Table 6.21 provide the mean PNC values corresponding to the SCL lengths recommended by AASHTO (2011) and TAC (2017) respectively. Few comparisons and observations made from Table 6.20 and Table 6.21 are

- The SCL lengths recommended by TAC (2017) were higher than those recommended by AASHTO (2011) for the same entrance curve design speed. Due to this reason, same values of entrance curve design speed, FRL speed, and FRL traffic volume, the SCL lengths recommended by TAC (2017) provide lower mean PNC values than those recommended by AASHTO (2011).

- For all the combinations of input parameters in all three methods, the mean PNC increased with the increase in the FRL traffic volume and ramp controlling curve design speed. 
- Even though, the change in mean PNC was considerably low, there were few combinations for which the mean PNC was reduced from Method 1 to Method 2 and from Method 1 to Method 3.

Table 6.20: PNC Values for the Recommended SCL Lengths by AASHTO (2011) Guide.

\begin{tabular}{|c|c|c|c|c|c|c|c|c|c|c|}
\hline \multirow{3}{*}{$\begin{array}{c}\text { Ramp } \\
\text { Controlling } \\
\text { Curve } \\
\text { Design } \\
\text { Speed } \\
(\mathbf{k m} / \mathbf{h})\end{array}$} & \multirow{3}{*}{$\begin{array}{c}\text { FRL } \\
\text { Speed } \\
(\mathbf{k m} / \mathbf{h})\end{array}$} & \multicolumn{9}{|c|}{ Traffic Volume (veh/h) } \\
\hline & & \multicolumn{3}{|c|}{400} & \multicolumn{3}{|c|}{800} & \multicolumn{3}{|c|}{1200} \\
\hline & & $\mathbf{M}_{1}$ & $\mathbf{M}_{2}$ & $\mathbf{M}_{3}$ & $\mathbf{M}_{1}$ & $\mathbf{M}_{2}$ & $\mathbf{M}_{3}$ & $\mathbf{M}_{1}$ & $\mathbf{M}_{2}$ & $\mathbf{M}_{3}$ \\
\hline \multicolumn{11}{|c|}{ Recommended SCL Length $(\mathrm{m})=460$} \\
\hline \multirow{3}{*}{50} & 90 & 0.00245 & 0.00237 & 0.00226 & 0.00646 & 0.00605 & 0.00605 & 0.01573 & 0.01339 & 0.01449 \\
\hline & 100 & 0.00197 & 0.00195 & 0.00216 & 0.00573 & 0.00483 & 0.00469 & 0.01588 & 0.01235 & 0.01527 \\
\hline & 110 & 0.00177 & 0.00197 & 0.00179 & 0.00395 & 0.00365 & 0.00402 & 0.01380 & 0.01066 & 0.01361 \\
\hline \multirow{3}{*}{60} & 90 & 0.00870 & 0.00862 & 0.00852 & 0.01919 & 0.01854 & 0.01910 & 0.03799 & 0.03548 & 0.03975 \\
\hline & 100 & 0.00824 & 0.00795 & 0.00802 & 0.01859 & 0.01763 & 0.01861 & 0.04224 & 0.03660 & 0.04319 \\
\hline & 110 & 0.00757 & 0.00753 & 0.00753 & 0.01716 & 0.01554 & 0.01610 & 0.03978 & 0.03244 & 0.03674 \\
\hline \multirow{3}{*}{70} & 90 & 0.02457 & 0.02413 & 0.02563 & 0.04626 & 0.04407 & 0.04557 & 0.08206 & 0.07333 & 0.08437 \\
\hline & 100 & 0.02441 & 0.02423 & 0.02461 & 0.04723 & 0.04337 & 0.04672 & 0.08590 & 0.07503 & 0.08705 \\
\hline & 110 & 0.02352 & 0.02366 & 0.02377 & 0.04426 & 0.04136 & 0.04330 & 0.08062 & 0.06855 & 0.07784 \\
\hline \multicolumn{11}{|c|}{ Recommended SCL Length $(\mathrm{m})=410$} \\
\hline \multirow{3}{*}{50} & 90 & 0.00251 & 0.00246 & 0.00260 & 0.00782 & 0.00717 & 0.00854 & 0.02327 & 0.02015 & 0.02273 \\
\hline & 100 & 0.00220 & 0.00223 & 0.00218 & 0.00763 & 0.00639 & 0.00688 & 0.02399 & 0.02042 & 0.02348 \\
\hline & 110 & 0.00205 & 0.00193 & 0.00197 & 0.00718 & 0.00620 & 0.00603 & 0.02425 & 0.01867 & 0.02161 \\
\hline \multirow{3}{*}{60} & 90 & 0.00951 & 0.00942 & 0.00937 & 0.02593 & 0.02183 & 0.02390 & 0.05457 & 0.04785 & 0.05260 \\
\hline & 100 & 0.00940 & 0.00923 & 0.00911 & 0.02537 & 0.02338 & 0.02377 & 0.06255 & 0.04959 & 0.05305 \\
\hline & 110 & 0.00913 & 0.00924 & 0.00919 & 0.02229 & 0.02121 & 0.02180 & 0.05808 & 0.04788 & 0.05193 \\
\hline \multirow{3}{*}{70} & 90 & 0.02665 & 0.02637 & 0.02607 & 0.05450 & 0.05110 & 0.05318 & 0.10238 & 0.09333 & 0.09832 \\
\hline & 100 & 0.02677 & 0.02662 & 0.02658 & 0.05727 & 0.05350 & 0.05489 & 0.10962 & 0.09533 & 0.10250 \\
\hline & 110 & 0.02667 & 0.02700 & 0.02698 & 0.05458 & 0.05082 & 0.05345 & 0.10609 & 0.09230 & 0.09671 \\
\hline \multicolumn{11}{|c|}{ Recommended SCL Length $(\mathrm{m})=325$} \\
\hline \multirow{3}{*}{50} & 90 & 0.00404 & 0.00380 & 0.00374 & 0.01567 & 0.01361 & 0.01523 & 0.04728 & 0.03988 & 0.04643 \\
\hline & 100 & 0.00402 & 0.00385 & 0.00377 & 0.01686 & 0.01409 & 0.01441 & 0.04998 & 0.04181 & 0.04442 \\
\hline & 110 & 0.00426 & 0.00398 & 0.00405 & 0.01881 & 0.01555 & 0.01723 & 0.05361 & 0.04625 & 0.04881 \\
\hline \multirow{3}{*}{60} & 90 & 0.01366 & 0.01273 & 0.01280 & 0.04162 & 0.03844 & 0.03964 & 0.09820 & 0.08162 & 0.08973 \\
\hline & 100 & 0.01450 & 0.01303 & 0.01300 & 0.04579 & 0.04013 & 0.04076 & 0.10135 & 0.08528 & 0.09672 \\
\hline & 110 & 0.01555 & 0.01391 & 0.01452 & 0.04761 & 0.04201 & 0.04350 & 0.10700 & 0.09170 & 0.09553 \\
\hline \multirow{3}{*}{70} & 90 & 0.03312 & 0.03094 & 0.03215 & 0.08380 & 0.07234 & 0.07863 & 0.15568 & 0.14285 & 0.14706 \\
\hline & 100 & 0.03577 & 0.03312 & 0.03247 & 0.08637 & 0.07681 & 0.07398 & 0.16284 & 0.14459 & 0.15563 \\
\hline & 110 & 0.03943 & 0.03588 & 0.03511 & 0.09019 & 0.08088 & 0.08200 & 0.17196 & 0.14869 & 0.15256 \\
\hline
\end{tabular}


Table 6.21: PNC Values for the Recommended SCL Lengths by TAC (2017) Guide.

\begin{tabular}{|c|c|c|c|c|c|c|c|c|c|c|}
\hline \multirow{3}{*}{$\begin{array}{c}\text { Ramp } \\
\text { Controlling } \\
\text { Curve } \\
\text { Design } \\
\text { Speed } \\
\text { (km/h) }\end{array}$} & \multirow{3}{*}{$\begin{array}{c}\text { FRL } \\
\text { Speed } \\
(\mathbf{k m} / \mathbf{h})\end{array}$} & \multicolumn{9}{|c|}{ Traffic Volume (veh/h) } \\
\hline & & \multicolumn{3}{|c|}{400} & \multicolumn{3}{|c|}{800} & \multicolumn{3}{|c|}{1200} \\
\hline & & $\mathbf{M}_{1}$ & $\mathbf{M}_{2}$ & $\mathbf{M}_{3}$ & $\mathbf{M}_{1}$ & $\mathbf{M}_{2}$ & $\mathbf{M}_{3}$ & $\mathbf{M}_{1}$ & $\mathbf{M}_{2}$ & $\mathbf{M}_{3}$ \\
\hline \multicolumn{11}{|c|}{ Recommended SCL Length $(\mathrm{m})=660$} \\
\hline \multirow{3}{*}{50} & 90 & 0.00172 & 0.00174 & 0.00178 & 0.00245 & 0.00238 & 0.00249 & 0.00304 & 0.00264 & 0.00279 \\
\hline & 100 & 0.00155 & 0.00150 & 0.00165 & 0.00211 & 0.00195 & 0.00177 & 0.00287 & 0.00271 & 0.00318 \\
\hline & 110 & 0.00143 & 0.00143 & 0.00137 & 0.00156 & 0.00157 & 0.00161 & 0.00275 & 0.00260 & 0.00300 \\
\hline \multirow{3}{*}{60} & 90 & 0.00711 & 0.00684 & 0.00718 & 0.00950 & 0.00956 & 0.00996 & 0.01141 & 0.01074 & 0.01126 \\
\hline & 100 & 0.00659 & 0.00663 & 0.00686 & 0.00864 & 0.00900 & 0.00976 & 0.01227 & 0.00983 & 0.01141 \\
\hline & 110 & 0.00597 & 0.00607 & 0.00627 & 0.00819 & 0.00735 & 0.00801 & 0.01122 & 0.00984 & 0.01122 \\
\hline \multirow{3}{*}{70} & 90 & 0.02109 & 0.02108 & 0.02178 & 0.02820 & 0.02802 & 0.02880 & 0.03473 & 0.03191 & 0.03342 \\
\hline & 100 & 0.02018 & 0.02085 & 0.02080 & 0.02651 & 0.02643 & 0.02703 & 0.03519 & 0.03123 & 0.03556 \\
\hline & 110 & 0.01978 & 0.01931 & 0.02007 & 0.02378 & 0.02437 & 0.02437 & 0.03380 & 0.02951 & 0.03157 \\
\hline \multicolumn{11}{|c|}{ Recommended SCL Length $(\mathrm{m})=\mathbf{5 9 0}$} \\
\hline \multirow{3}{*}{50} & 90 & 0.00198 & 0.00209 & 0.00190 & 0.00323 & 0.00319 & 0.00301 & 0.00523 & 0.00447 & 0.00496 \\
\hline & 100 & 0.00173 & 0.00173 & 0.00167 & 0.00255 & 0.00269 & 0.00243 & 0.00504 & 0.00451 & 0.00551 \\
\hline & 110 & 0.00144 & 0.00149 & 0.00142 & 0.00196 & 0.00184 & 0.00199 & 0.00518 & 0.00370 & 0.00447 \\
\hline \multirow{3}{*}{60} & 90 & 0.00747 & 0.00737 & 0.00761 & 0.01174 & 0.01186 & 0.01219 & 0.01709 & 0.01513 & 0.01659 \\
\hline & 100 & 0.00727 & 0.00676 & 0.00683 & 0.01070 & 0.01077 & 0.01091 & 0.01856 & 0.01533 & 0.01669 \\
\hline & 110 & 0.00625 & 0.00638 & 0.00641 & 0.00966 & 0.00928 & 0.00882 & 0.01500 & 0.01477 & 0.01539 \\
\hline \multirow{3}{*}{70} & 90 & 0.02226 & 0.02193 & 0.02266 & 0.03216 & 0.03249 & 0.03196 & 0.04621 & 0.04084 & 0.04373 \\
\hline & 100 & 0.02199 & 0.02121 & 0.02160 & 0.03136 & 0.03065 & 0.03204 & 0.04606 & 0.04065 & 0.04413 \\
\hline & 110 & 0.02094 & 0.02058 & 0.02091 & 0.02890 & 0.02790 & 0.02892 & 0.04302 & 0.03817 & 0.04025 \\
\hline \multicolumn{11}{|c|}{ Recommended SCL Length $(m)=515$} \\
\hline \multirow{3}{*}{50} & 90 & 0.00191 & 0.00180 & 0.00197 & 0.00439 & 0.00724 & 0.00421 & 0.00936 & 0.02210 & 0.00953 \\
\hline & 100 & 0.00145 & 0.00160 & 0.00157 & 0.00362 & 0.00318 & 0.00372 & 0.00832 & 0.00760 & 0.00841 \\
\hline & 110 & 0.00160 & 0.00161 & 0.00160 & 0.00267 & 0.00288 & 0.00287 & 0.00820 & 0.00664 & 0.00875 \\
\hline \multirow{3}{*}{60} & 90 & 0.00724 & 0.00392 & 0.00731 & 0.01475 & 0.01435 & 0.01445 & 0.02788 & 0.03676 & 0.02662 \\
\hline & 100 & 0.00681 & 0.00681 & 0.00665 & 0.01349 & 0.01306 & 0.01340 & 0.02922 & 0.02225 & 0.02458 \\
\hline & 110 & 0.00701 & 0.00692 & 0.00721 & 0.01203 & 0.01148 & 0.01259 & 0.02466 & 0.02164 & 0.02325 \\
\hline \multirow{3}{*}{70} & 90 & 0.02203 & 0.00767 & 0.02204 & 0.03825 & 0.02450 & 0.03752 & 0.06128 & 0.05552 & 0.06031 \\
\hline & 100 & 0.02048 & 0.02094 & 0.02136 & 0.03710 & 0.03450 & 0.03722 & 0.06302 & 0.05655 & 0.06240 \\
\hline & 110 & 0.02189 & 0.02145 & 0.02229 & 0.03457 & 0.03450 & 0.03504 & 0.06332 & 0.05340 & 0.05604 \\
\hline
\end{tabular}


From the findings of all the above sections, it could be noticed that CVs can decrease the PNC of SCL vehicles at the assumed conditions in this study. Collision frequencies on freeway acceleration SCLs were decreased with the advantages of CVs, thus improving the safety at freeway entrance ramps. Hence vehicle connectivity is capable of improving the safety performance of freeway entrance ramps after the deployment of CVs. 


\section{Chapter 7: CONCLUSIONS}

This study presented detailed description of simulation techniques to model the driver behaviour on freeway entrance SCLs merging into freeway through traffic, considering the gap searching behaviour of SCL drivers. Simulation methods were developed to replicate the vehicles movement in the absence of vehicle connectivity and in the presence of vehicle connectivity. Monte Carlo simulation method was used to account for the variability in various input parameters such as vehicle speed, acceleration, and acceptable gap characteristics based on the available field data. Probabilistic design approach was used in which the distributions of input parameters were considered instead of a single value. For the specific characteristics of each selected study site, the mean probability of uncomfortable merging was calculated for all SCL vehicles simulated in all methods and this was termed as the probability of non-compliance (PNC). The changes in mean PNC were examined in the CV environment, assuming a penetration rate of $100 \%$ for CVs.

\subsection{Findings}

The findings from this research work are listed below:

1. The benefits V2V applications, could be utilized to decrease the probability of uncomfortable merging of acceleration lane drivers, assuming the conditions described in this study.

2. Both methods, co-operative merging (Method-2) and lane changing request (Method -3) exhibited a decrease in the mean PNC at the study SCL sites, when compared with the mean PNC results from Method-1. 
3. From the sensitivity analysis, it was found that the mean PNC was more sensitive to the input parameters such as initial speed, SCL length, FRL traffic volume. This means that the increase or decrease in these parameters would have a considerable effect on the probability of uncomfortable merging at the freeway entrance areas, thus effecting the efficiency of the entrance acceleration SCLs.

4. A collision prediction model was developed relating the collision frequency to the traffic exposure and mean PNC. Predicted collisions at the study sites were calculated using the developed CPM and the mean PNC from the three methods. A decrease in the predicted collision frequency was observed in Method-2 and Method-3. Thus, demonstrating the benefit of using PNC in predicting the collision frequency. Considering the collision frequency as a surrogate safety measure, the decrease in the collision frequency indicates the improvement in the safety the freeway merging areas.

5. Mean PNC values for the acceleration lane lengths recommended by the design guides AASHTO (2011) and TAC (2017) for various combinations of input parameters were also evaluated and the results were found similar to the results of sensitivity analysis.

\subsection{Further Research Recommendations}

Following are the recommendations to be considered for future research work:

1. The present study considered only 16 SCL sites along Highway 417 located in the City of Ottawa. The number of study sites need to be increased and different 
locations along the same highway or different highways can be considered to properly understand the variations in driver's behaviour.

2. Speed data on the freeway second lane were not available and the same should be collected and used to improve the accuracy of the simulation.

3. At the selected study sites, speed data were available only from the gore area to the merging location. However, speed data from the beginning of the entrance curve would help in accurate representation of SCL driver's acceleration and gap searching behaviour.

4. Traffic volume data on freeway second lane at some of the study sites were not available. Traffic volume data on F2L would assist in accurately calculating the headways of F2L vehicles, and thus in accurately positioning the F2L vehicles.

5. In this study, the penetration rate of CVs was considered as $100 \%$. However, different percentage of penetration rates should be considered for evaluation purposes. Also, mixed environment of vehicles such as regular vehicles, connected vehicles, and autonomous vehicles should also be considered in the future research.

6. The data available from past research studies were used in this study. However, real-world data about CVs behaviour should be used to improve accuracy of the results and to observe the real benefits of $\mathrm{CVs}$ in improving the safety at freeway entrance ramps.

7. While sending the deceleration request or lane change request for the FRL vehicles, only interactions with the lag vehicle were considered. However, interactions with the lead vehicles should also be included in further research. 
8. Cooperative merging in regular vehicles was ignored and this could be considered in future research. In the simulation of CVs, when the FRL driver receives a deceleration request or a lane change request, the driver either can accept the request or reject it. In the simulation of merging process in $\mathrm{CV}$ environment, a request to decelerate or to change the lane was sent to FRL lag vehicle, only when it is safe to do so. Hence, it was assumed that there will be $100 \%$ compliance with the deceleration or lane change request with CVs and this can be varied in further research. 


\section{REFERENCES}

AASHTO. (1965). A Policy on Geometric Design of Highways and Streets. American Association of State Highway and Transportation Officials. Washington, D.C.

AASHTO. (2004). A Policy on Geometric Design of Highways and Streets. American Association of State Highway and Transportation Officials. Washington, D.C.

AASHTO. (2011). A Policy on Geometric Design of Highways and Streets. American Association of State Highway and Transportation Officials. Washington, D.C.

Ahammed, M. A. (2005). Freeway Merging Behaviour And Safety of Acceleration Lanes: Field Study. Carleton University, Ottawa, ON, Canada. https://doi.org/10.16953/deusbed.74839

Ahammed, M. A., Hassan, Y., \& Sayed, T. a. (2008). Modeling Driver Behavior and Safety on Freeway Merging Areas. Journal of Transportation Engineering, 134(September), 370-377. https://doi.org/10.1061/(ASCE)0733-947X(2008)134:9(370)

Ahmed, K. I. (1999). Modeling Drivers' Acceleration and Lane Changing Behavior. Massachusetts Institute of Technology.

Ben-Akiva, M., Hirsh, M., \& Prashker, J. (1985). Probabilistic and Economic Factors in Highway Geometric Design. Transportation Science, 19(1), 38-57. https://doi.org/10.1287/trsc.19.1.38

Brewer, M., Fitzpatrick, K., \& Stanley, J. (2011). Driver Behavior on Speed-Change Lanes at Freeway Ramp Terminals. Transportation Research Record: Journal of the Transportation Research Board, 2223(2223), 54-62. https://doi.org/10.3141/2223-07 
Campbell, R., Alexiadis, V., \& Krechmer, D. (2015). Connected Vehicle Impacts on Transportation Planning. Technical Memorandum\#3: Analysis of the Need for New and Enhanced Analysis Tools, Techniques, and Data.

Campbell, R., Alexiadis, V., \& Krechmer, D. (2016). Connected Vehicle Impacts on Transportation Planning. Analysis of the Need for New and Enhanced Analysis Tools, Techniques, and Data - Briefing for Traffic Simulation Models.

Chong, J. (2016). Automated and connected vehicles: Status of the technology and key policy issues for Canadian governments. Library of Parliament Research Publication. Retrieved from http://www.lop.parl.gc.ca/Content/LOP/ResearchPublications/201698-e.html

Dhahir, B. (2018). Reliability-Based, Safety-Explicit Horizontal Curve Design Using Naturalistic Driving Study. Carleton University, Ottawa, Ontario.

Easa, S. (2000). Reliability Approach to Intersection Sight Distance Design. Transportation Research Record: Journal of the Transportation Research Board, 1701(April), 42-52. https://doi.org/10.3141/1701-06

Fatema, T. (2012). Probabilistic Design of Freeway Entrance Speed Change Lanes Considering Acceleration and Gap Acceptance Behaviour. Carleton University, Ottawa, Ontario, Canada.

Fatema, T., \& Hassan, Y. (2013). Probabilistic Design of Freeway Entrance Speed-Change Lanes Considering Acceleration and Gap Acceptance Behavior. Transportation Research Record: Journal of the Transportation Research Board, 2348, 30-37. 
https://doi.org/10.3141/2348-04

Fatema, T., Ismail, K., \& Hassan, Y. (2014). Validation of Probabilistic Model for Design of Freeway Entrance Speed Change Lanes. Transportation Research Record: Journal of the Transportation Research Board, 2460, 97-106. https://doi.org/10.3141/246011

Fitzpatrick, K., \& Zimmerman, K. H. (2007). Potential Updates to 2004 Green Book’s Acceleration Lengths for Entrance Terminals. Transportation Research Record: Journal of the Transportation Research Board, (2023), pp 130-139. https://doi.org/10.3141/2023-14

Garber, N. J., \& Hoel, L. A. (2009). Traffic \& Highway Engineering (Fourth Edi). Cengage Learning.

Geometric Design Standards for Ontario Highways. (1994). Ann Arbor: ProQuest Micromedia.

Gipps, P. G. (1986). A MODEL FOR THE STRUCTURE OF LANE-CHANGING DECISIONS. Transportation Research Board, 20B, 403-414.

Goodall, N. J., Smith, B. L., \& Park, B. B. (2016). Microscopic Estimation of Freeway Vehicle Positions from the Behavior of Connected Vehicles. Journal of Intelligent Transportation Systems: Technology, Planning, and Operations, 20(1), 45-54. https://doi.org/10.1080/15472450.2014.889926

Guériau, M., Billot, R., El Faouzi, N. E., Monteil, J., Armetta, F., \& Hassas, S. (2016). How to assess the benefits of connected vehicles? A simulation framework for the 
design of cooperative traffic management strategies. Transportation Research Part C: Emerging Technologies, 67, 266-279. https://doi.org/10.1016/j.trc.2016.01.020

Hassan, Y., Sarhan, M., \& Salehi, M. (2012). Probabilistic Model for Design of Freeway Acceleration Speed-Change Lanes. Transportation Research Record: Journal of the Transportation Research Board, 2309(1), 3-11. https://doi.org/10.3141/2309-01

Hayat, M. T., Park, H., \& Smith, B. L. (2016). Investigating Driver Response Time to Freeway Merge Advisories in a Connected Vehicle Environment. Transportation Research Record: Journal of the Transportation Research Board, 2559, 131-140. https://doi.org/10.3141/2559-15

Hill, C., Hamilton, A., \& Krueger, G. (2013). Module 13: Connected Vehicles. Retrieved from https://www.pcb.its.dot.gov/eprimer/documents/module13.pdf

Hunter Michael, Machemehl Randy, \& Tsyganov Alexei. (2001). Operational Evaluation of Freeway Ramp Design. Transportation Research Record, 1751.

Hwang, S. Y., \& Park, C. H. (2005). Modeling of the Gap Acceptance Behavior at a Merging Section of Urban Freeway. Proceedings of the Eastern Asia Society for Tansportation Studies, 5, 1641-1656.

Ismail, K., \& Sayed, T. (2009). Risk-based framework for accommodating uncertainty in highway geometric design. Canadian Journal of Civil Engineering, 36(5), 743-753. https://doi.org/10.1139/L08-146

Jadaan, K., Zeater, S., \& Abukhalil, Y. (2017). Connected Vehicles: an Innovative Transport Technology. Procedia Engineering, 187, 641-648. 
https://doi.org/10.1016/j.proeng.2017.04.425

Khoury, J., \& Hobeika, A. (2007). Assessing the risk in the design of passing sight distances. Journal of Transportation Engineering, 133(June), 370-377. https://doi.org/10.1061/(ASCE)0733-947X(2007)133:6(370)

Kim, J.-T., Kim, J., \& Chang, M. (2008). Lane-changing gap acceptance model for freeway merging in simulation. Canadian Journal of Civil Engineering, 35(3), 301-311. https://doi.org/10.1139/L07-119

Kita, H. (1999). A merging-giveway interaction model of cars in a merging section: a game theoretic analysis.

Li, W., Wang, W., Chen, J., \& Li, X. (2007). Field study and modeling of vehicles' merging behavior from an acceleration lane to expressway traffic. Advances in Transportation Studies, 12(12), 59-70.

Liz, G., Janet, F., Drennan, H., Mike, M., \& Kathy, T. (2018). Intelligent Transportation Systems Benefits, Costs, Deployment, and Lessons Learned 2018 Update Report. Fhwa-Op (Vol. 05-002).

Maurya, A. K., \& Bokare, P. S. (2012). Study of Deceleration Behaviour of Different Vehicle Types. International Journal for Traffic and Transport Engineering, 2(3), 253-270. https://doi.org/10.7708/ijtte.2012.2(3).07

National Highway Traffic Safety Administration (NHSTA). (2018). Summary of motor vehicle crashes: 2016 data, (August), 1-8. Retrieved from https://crashstats.nhtsa.dot.gov/Api/Public/ViewPublication/812554 
Park, K. S., Lee, A. J., \& Koh, B. K. (2001). Drivers' Characteristics in the Perception of a Lead Vehicle's Deceleration Level. International Journal of Cognitive Ergonomics, 5(2), 125-136. https://doi.org/10.1207/S15327566IJCE0502_3

Polus, B. A., Livneh, M., \& Factor, J. (1985). Vehicle flow characteristics on acceleration lanes, $I(6), 595-606$.

Richl, L., \& Sayed, T. (2006). Evaluating the Safety Risk of Narrow Medians Using Reliability Analysis. Journal of Transportation Engineering, 132(5), 366-375. https://doi.org/10.1061/(ASCE)0733-947X(2006)132:5(366)

Salehi, M. (2010). Reliability-Based Design of Freeway Acceleration Speed - Change Lanes. Carleton University, Ottawa, ON, Canada.

Sarhan, M. (2004). Safety Performance of Freeway Merge and Diverge Areas. Carleton University, Ottawa, ON, Canada.

Sarhan, M. (2008). Risk-based approach for highway geometric design. Carleton University, Ottawa, Ontario.

Sayed, T., \& Leur, P. de. (2008). Collision Prediction Models For British Columbia. BC Ministry of Transportation \& Infrastructure Prepared, (December), 31.

Schrank, D., Lomax, T., \& Eisele, B. (2011). The 2011 urban mobility report, (September). Retrieved from http://rid.trb.org/view.aspx?id=1122263

Seneviratne, P. N., \& Islam, M. N. (1992). Speed Estimates for Roadway Design and Traffic Control. Transportation Research Record: Journal of the Transportation 
Research Board, 1375, 37-43.

Singh, V. P., Jain, S. K., \& Tyagi, A. (2007). Risk and Reliability Analysis: A handbook for Civil and Environmental Engineers. Virginia: ASCE Press.

Smith, B. L., Park, H., Malathi, V., \& Park, B. B. (2012). Advanced Freeway Merge Assistance: Harnessing the Potential of Connected Vehicles.

Smith, B. L., Park, H., \& Shize, S. (2014). A Prototype Freeway Merging Control Algorithm Under a Connected Vehicle Environment.

TAC. (2017). Geometric Design Guide for Canadian Roads. Transportation Association of Canada. Ottawa, Ontario, Canada.

Talebpour, A., \& Hani, M. S. (2014). MODELING ACCELERATION BEHAVIOR IN A CONNECTED ENVIRONMENT, (1), 1-4.

Thompson, A., \& Kent, G. (2018). Connect Vehicle Pilot Success in Ottawa Eco Drive I2V Proof of Concept Project. In ITS Canada Conference.

Transport Canada. (2018). Canadian Motor Vehicle Traffic Collision Statistics: 2016. https://doi.org/10.1016/S0306-4603(02)00297-6

US Census Bureau. (2004). 2002 Economic Census: Vehicle Inventory and Use Survey: United States : 2002. US Census Bureau, (December).

USDOT. (2018). Connected Vehicle Deployment Program. United States Department of Transportation - Intelligent Transportation Systems. Retrieved November 14, 2018, from https://www.its.dot.gov/pilots/pilots_overview.htm 
Xiaosi, Z., Kevin, B., \& Praprut, S. (2012). Potential Connected Vehicle Applications to Enhance Mobility, Safety and Environmental Security (Vol. 7). Texas.

Yang, G., Tian, Z., Xu, H., \& Wang, Z. (2016). Recommendations for Acceleration Lane Length for Metered On-Ramps. Transportation Research Record: Journal of the Transportation Research Board, 2588(December), 1-11. https://doi.org/10.3141/2588-01

Yang Qi, \& Haris, K. N. (1996). full-text. A Microscopic Traffic Simulator for Evaluation of Dynamic Traffic Mnagament Systems, 4(3), 113-129.

Zhang, G., Wang, Y., Wei, H., \& Chen, Y. (2007). Examining Headway Distribution Models with Urban Freeway Loop Event Data. Transportation Research Record: Journal of the Transportation Research Board, 1999, 141-149. https://doi.org/10.3141/1999-15

Zhao, D., Peng, H., Bao, S., Nobukawa, K., LeBlanc, D. J., Pan, C. S., ... Pan, C. S. (2014). Analysis of Mandatory and Discretionary Lane Change Behaviors for Heavy Trucks. 14th International Symposium on Advanced Vehicle Control, (Mlc), 1-6. https://doi.org/10.1161/01.RES.64.6.1158 


\section{APPENDIX A}

Freeway Lag Vehicle Critical Headway and Speed Adjustments (Equations 5.3 and 5.5) 


\section{Critical Headway Calculation for the Freeway Lag Vehicle:}

Figure A.1 provides the positions of lag and lead vehicles after the displacements of $\mathrm{X}_{1}$ and $\mathrm{X}_{2}$ respectively.

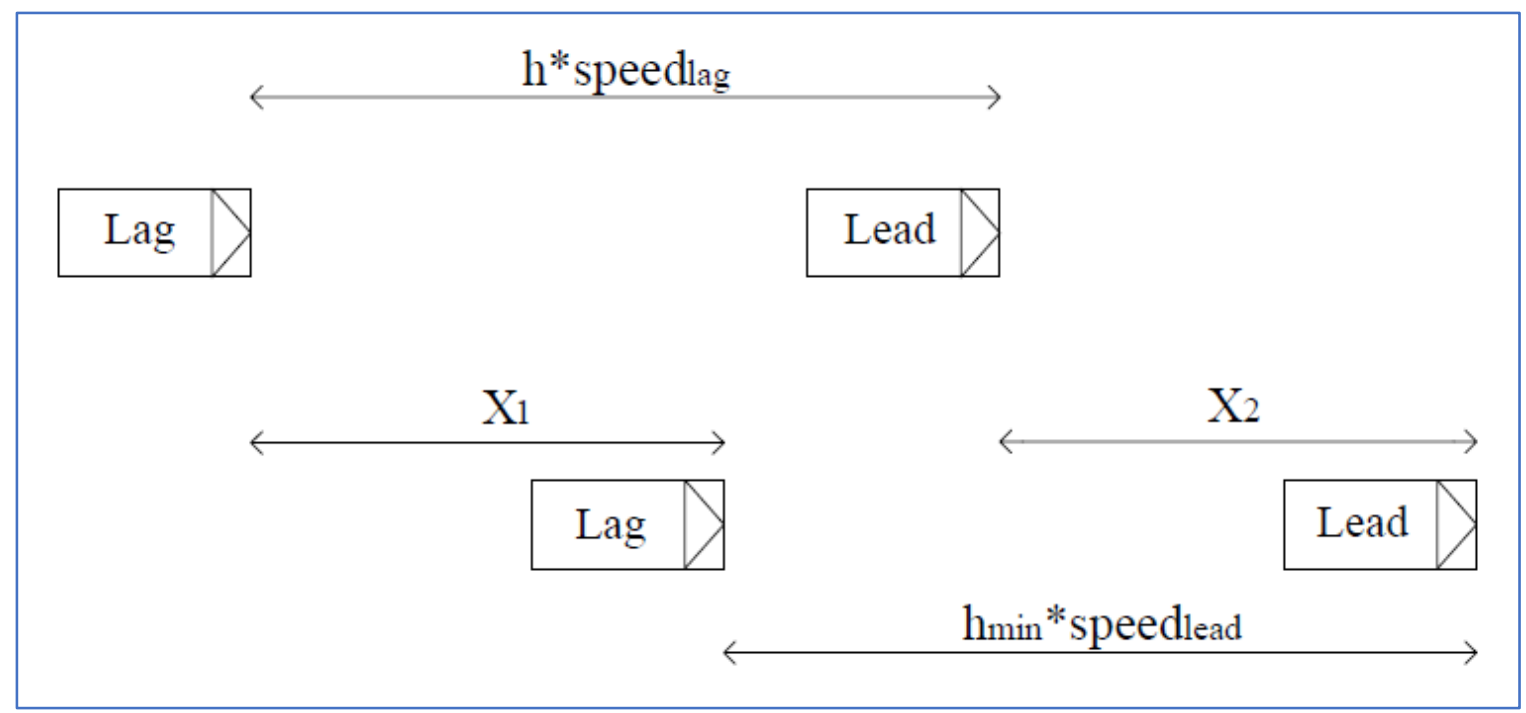

Figure A.1: Vehicle Positions for Critical Headway Calculation

$$
\begin{aligned}
& X_{1}+h_{\text {min }} \text { speed }_{\text {lag }}=X_{2}+h_{\text {crit }} \text { speed }_{\text {lag }} \\
& X_{1}=\frac{\text { speed }_{\text {lag }}^{2}-\text { speed }_{\text {lead }}^{2}}{2 * \text { decRate }_{\text {lag }}} ; X_{2}=\text { speed }_{\text {lead }}\left(\frac{\text { speed }_{\text {lag }}-\text { speed }_{\text {lead }}}{\text { decRate }_{\text {lag }}}\right)
\end{aligned}
$$

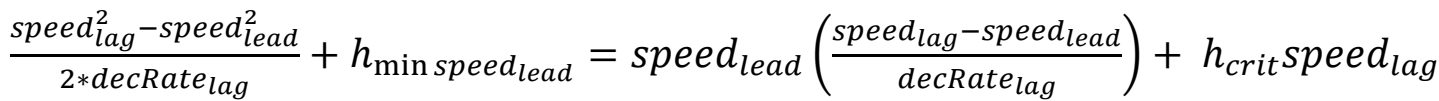

$$
\begin{aligned}
& \left(\frac{\left(\text { speed }_{\text {lag }}-\text { speed }_{\text {lead }}\right)\left(\text { speed }_{\text {lag }}+\text { speed }_{\text {lead }}\right)}{2 \text { decRate }_{\text {lag }}}\right)-2 \text { speed }_{\text {lead }}\left(\frac{\text { speed }_{\text {lag }}-\text { speed }_{\text {lead }}}{2 \text { decRate }_{\text {lag }}}\right)= \\
& h_{\text {crit }} \text { speed } d_{\text {lag }}-h_{\text {min }} \text { speed } d_{\text {lead }} \\
& \left(\frac{\text { speed }_{\text {lag }}-\text { speed }_{\text {lead }}}{2 \text { decRate }_{\text {lag }}}\right)\left(\text { speed }_{\text {lag }}-\text { speed }_{\text {lead }}\right)=h_{\text {crit }} \text { speed }_{\text {lag }}-h_{\min \text { speed }} \text { lead }
\end{aligned}
$$




$$
\frac{\left(\text { speed }_{\text {lag }}-\text { speed }_{\text {lead }}\right)^{2}}{2 \text { decRate }_{\text {lag }}}=h_{\text {crit }} \text { speed }_{\text {lag }}-h_{\text {min }} \text { speed }_{\text {lead }}
$$

$$
\begin{gathered}
\text { decRate }_{\text {lag }}=\frac{\left(\text { speed }_{\text {lag }}-\text { speed }_{\text {lead }}\right)^{2}}{2\left(h_{\text {crit }} \text { speed }_{\text {lag }}-h_{\text {min }} \text { speed }_{\text {lead }}\right)} \\
h_{\text {crit }}=\frac{\left(\text { speed }_{\text {lag }}-\text { speed }_{\text {lead }}\right)^{2}}{2 * \text { decRate }_{\text {lag }} \text { speed }_{\text {lag }}}+\frac{h_{\text {min }_{\text {speed }}} \text { lead }}{\text { speed }_{\text {lag }}}
\end{gathered}
$$

\section{Adjustment Required for the Speed of the Lag Vehicle:}

$$
\begin{aligned}
& X_{1}+h_{\text {min }} * \text { speed }_{\text {lead }}=X_{2}+h * \text { speed }_{\text {lag }} \\
& X_{1}+h_{\text {min }} * \text { speed }_{\text {lead }}=\text { speed }_{\text {lead }} * t+h * \text { speed }_{\text {lag }} \\
& X_{1}=\operatorname{speed}_{\text {lead }} * t-h_{\text {min }} * \text { speed }_{\text {lead }}+h * \text { speed }_{\text {lag }} \\
& X_{1}=\text { speed }_{\text {lead }}\left[\frac{\text { speed }_{\text {lag }}-\text { speed }_{\text {lead }}}{\text { decRate }_{\text {lag }}}\right]-h_{\text {min }} * \text { speed }_{\text {lead }}+h * \text { speed }_{\text {lag }} \\
& X_{1}=\frac{\text { speed }_{\text {lead }} * \text { speed }_{\text {lag }}}{\text { decRate }_{\text {lag }}}-\frac{\text { speed }_{\text {lead }}^{2}}{\text { decRate }_{\text {lag }}}-h_{\text {min }} * \operatorname{speed}_{\text {lead }}+h * \operatorname{speed}_{\text {lag }} \\
& \text { speed }_{\text {lag }}^{2}=\text { speed }_{\text {lead }}^{2}+2 * X_{1} * \text { decRate }_{\text {lag }} \\
& \operatorname{speed}_{\text {lag }}^{2}=\operatorname{speed}_{\text {lead }}^{2}+\left(2 * \text { speed }_{\text {lead }} * \text { speed }_{\text {lag }}\right)-2 * \operatorname{speed}_{\text {lead }}^{2}-\left(2 * h_{\text {min }}\right. \\
& \left.* \text { speed }_{\text {lead }}\right)+\left(2 * h * \text { decRate }_{\text {lag }} * \text { speed }_{\text {lag }}\right) \\
& \operatorname{speed}_{\text {lag }}^{2}=\operatorname{speed}_{\text {lag }}\left(2 * \operatorname{speed}_{\text {lead }}+2 * h * \text { decRate }_{\text {lag }}\right) \\
& -\left(\operatorname{speed}_{\text {lead }}^{2}+2 * \operatorname{decRate}_{\text {lag }} * h_{\text {min } \text { speed }_{\text {lead }}}\right)
\end{aligned}
$$




$$
\begin{aligned}
& \text { speed }_{\text {lag }}^{2}-\text { speed }_{\text {lag }}\left(2 * \text { speed }_{\text {lead }}+2 * h * \text { decRate }_{\text {lag }}\right) \\
& +\left(\text { speed }_{\text {lead }}^{2}+2 * \text { decRate }_{\text {lag }} * h_{\text {min }} \text { speed }_{\text {lead }}\right)=0
\end{aligned}
$$

Solving the quadratic equation with $a=1, b=-2\left(\operatorname{speed}_{\text {lead }}+h * d\right)$,

$c=\operatorname{speed}_{\text {lead }}\left(\operatorname{speed}_{\text {lead }}+2 * d * h_{\text {min }}\right)$ for the roots yields the following:

speed $_{\text {lag }}=\frac{-b \pm \sqrt{b^{2}-4 a c}}{2 a}$

The positive root was considered, which gives the scenario of lag vehicle moving at a higher speed than its lead vehicle. 


\section{APPENDIX B}

MATLAB Codes for Vehicles Simulation and PNC Evaluation 
clear

clc

global analMethod nRuns

global lengthPC lengthHV

global warmupTime timeStep

global meanFrlSpeed sdFrlSpeed minFrlHeadway

global meanF21Speed sdF21Speed minF21Headway

global maxReqGap minSclHeadway

global goreLocation sclLength sclRampCurveToGore distanceFromGore addonDist

$\operatorname{rng}($ 'shuffle')

$\% \% \% \% \% \%$.......Selecting the analysis method.......\%\% \% $\% \% \% \%$

$\%$ analMethod $=1:$ SCL vehicles are released at their expected cum headway;

$\%$ gaps are calculated as PNC for the vehicle is being estimated

$\%$ analMethod $=1$ : the gaps are found and stored in one matrix to be used by

$\%$ the function TotalPNC

codeVer $=23 ; \%$ version of code used

allAnalMethods = [lll $\left.\begin{array}{ll}1 & 3\end{array}\right]$; \%all analysis methods; can be one value or a vector allNRuns $=$ [2]; \%all number of runs; can be one value or a vector

outputOption $=0 ; \%$ option to write output in MAT file: 0 or -ve do not write; +ve value write it

$\% \% \% \% \% \%$...... Setting up geometric and simulation parameters ....... $\% \% \% \% \% \% \% \% \% \%$

sclLength $=[400] ; \%$ total SCL length

goreLocation $=0 ; \%$ setting gore point as the zero station; all other locations are relative to this point

sclRampCurveToGore $=50 ; \%$ part of SCL length from end of ramp controlling curve to gore assumed as $50 \mathrm{~m}$

distanceFromGore $=1500 ; \%$ distance considered behind gore location

addonDist $=500 ; \%$ an add-on distance after SCL where FRL vehicles are still simulated to capture their effect on vehicles within the SCL area

timeStep $=0.1 ; \%$ time step in the simulation

warmupTime $=5 * 60 ; \%$ warmup time in seconds ( $\mathrm{PNC}$ is not calculated during this time) 
$\% \% \% \% \% \% \% \% \% \% \% \% \%$

FRL Vehicles .$\% \% \% \% \% \% \% \% \% \% \% \% \% \% \% \% \%$

$\%$ FRL traffic volume \& characteristics

$\%$ Note 1: nSimFrlVeh is increased by warmuptime and $20 \%$ to ensure presence

$\%$ of FRL vehicles for all SCL vehicles

frlVolume $=[800] ; \%$ can be a vector of many values

minFrlHeadway $=0.5 ; \%$ min headway in FRL

meanFrlSpeed $=[102.16] / 3.6$; \%input mean FRL speed of all 16 sites $102.16 \mathrm{~km} / \mathrm{h}$

$\operatorname{sdFrlSpeed}=8.78 / 3.6 ; \%$ input mean SD of all 16 sites $8.78 \mathrm{~km} / \mathrm{h}$

$\mathrm{pHVFrl}=[0.1] ; \%$ proportion of heavy vehicles; can be a vector of many values

$\%$ FRL vehicle lengths

lengthHV $=12.5 ; \%$ all heavy vehicles have same length

lengthPC $=\left[\begin{array}{l}4.399 \\ 4.671 \\ 4.8364 .7894 .8774 .4354 .5974 .8724 .831 \ldots\end{array}\right.$

$5.0014 .4985 .094 .6714 .8315 .0014 .7894 .8774 .8344 .7894 .665 \ldots$

$4.7714 .7115 .2075 .0984 .8345 .1265 .131]$;

$\% \% \% \% \% \% \% \% \% \% \% \% \%$

$\%$ SCL traffic volume \& characteristics

sclVolume $=[400] ; \%$ can be a vector of many values

minSclHeadway $=0.5 ; \%$ min headway in SCL

gore85thSpeed=[70]./3.6; \%85th percentile gore speed $(\mathrm{km} / \mathrm{h})$; set it as the design speed of ramp controlling curve; can be a vector of many values

maxReqGap $=6 ; \%$ max gap that is likely to be accepted by all drivers

\%\%\%\%\%\%\%\%\%\%\%\%\%\%,...... F2L Vehicles .......\%\%\%\%\%\%\%\%\%\%\%\%\%\%\%\%

$\%$ Traffic volume \& characteristics of 2nd freeway lane

$\%$ Note 1: nSimF21Veh is increased by warmuptime and $20 \%$ to ensure presence

$\%$ of F2L vehicles for all SCL vehicles

\%Used only for analMethod 3

laneRatio = [1.5]; \%ratio of volume in F2L to FRL; can be a vector of many values

minF2lHeadway $=0.5 ; \%$ min headway in F2L

meanF21Speed $=[102.16] / 3.6$; \%input mean FRL speed of all 16 sites combined 102.16

$\mathrm{km} / \mathrm{h}$

sdF21Speed $=8.78 / 3.6 ; \%$ input SD of all 16 sites combined $8.78 \mathrm{~km} / \mathrm{h}$

pHVF2l $=[0.1] ; \%$ proportion of heavy vehicle; can be a vector of many values

\section{if outputOption}

outputFile = 'noRuns.mat';

Header $=\{$ 'Code Version',",", num2str(codeVer), ", ", ", ", ", ", "; ...

'SCL Length (m)',",", num2str(sclLength), ", ", ", ", ", ", "; ... 
'Mean FRL Speed (km/h)',",", num2str(meanFrlSpeed*3.6), ", ", ", ", ", ", "; ...

'Mean 2nd Lane Speed (km/h)',",", num2str(meanF21Speed*3.6), ", ", ", ", ", ", "; ... ", ", ", ", ", ", ", ", ", ", "; ...

'Index', 'analMethod', 'nRuns', 'FRL Vol', 'P_HV (FRL)', 'SCL Vol', 'V_G,85 (km/h)', $\cdots$

'Vol Ratio (2nd to RL)', 'P_HV (F2L)', 'meanPNC', 'sdPNC'\};

$\mathrm{N}=$

length(allAnalMethods)*length(allNRuns)*length(frlVolume)*length(pHVFrl)*length(sc IVolume)*length(gore85thSpeed)*length(laneRatio)*length(pHVF2l);

SummaryData $=\mathrm{NaN}(\mathrm{N}, 11)$;

end

iScen $=0$;

nScenarios $=$

length(allAnalMethods)*length(allNRuns)*length(frlVolume)*length(meanFrlSpeed)*le ngth(pHVFrl)*length(sclVolume)*length(gore85thSpeed)*length(laneRatio)*length(mea nF21Speed)*length(pHVF21);

totalNSclVeh $=\max ($ allNRuns) $* \max ($ sclVolume $)$;

AllData $=\mathrm{NaN}($ totalNSclVeh $+1, \mathrm{nScenarios})$;

$\operatorname{AllData}(1,:)=1: n S c e n a r i o s ;$

fprintf('Total number of analysis scenarios: \%d $\backslash n$ ', nScenarios);

for im $=1$ :length(allAnalMethods)

analMethod $=$ allAnalMethods $(\mathrm{im})$;

for ir $=1$ :length(allNRuns)

nRuns $=$ allNRuns(ir);

for ivr $=1$ :length(frlVolume)

for ihvr $=1$ :length $(\mathrm{pHVFrl})$

for ivs $=1$ :length(sclVolume)

for igs $=1$ :length(gore 85 thSpeed $)$

for ilr $=1$ :length(laneRatio)

for ihv2 = 1:length(pHVF21)

iScen $=$ iScen +1 ;

fprintf('\nScenario \%d: Analysis method \%d \& Total Runs \%d $\backslash n$ ',

iScen, analMethod, nRuns);

[nTotalSCLVeh, totalPNC] $=$ PncAccelerationCVs22

(frlVolume(ivr), pHVFrl(ihvr), sclVolume(ivs), gore85thSpeed(igs), laneRatio(ilr), pHVF21(ihv2));

$\operatorname{AllData}(2:$ length $($ totalPNC $)+1$, iScen $)=$ totalPNC; 


\section{if outputOption \\ meanPNC = mean (totalPNC);}

$\operatorname{sdPNC}=\operatorname{std}($ totalPNC);

SummaryData(iScen,:) $=$ [iScen, analMethod, nRuns,

frlVolume(ivr), pHVFrl(ihvr), sclVolume(ivs), gore85thSpeed(igs)*3.6, laneRatio(ilr), pHVF21(ihv2), meanPNC, sdPNC];

end

end

end
end
end
end
end
end
end

if outputOption

save(outputFile, 'Header', 'SummaryData', 'AllData', 'codeVer', 'sclLength', 'meanFr1Speed', 'meanF21Speed', '-v7.3');

end

fprintf ('end of script $\backslash n$ ') 
function [nTotalSCLVeh, totalPNC] = PncAccelerationCVs22 (frlVolume, pHVFrl, sclVolume, gore85thSpeed, laneRatio, pHVF21)

global analMethod nRuns

global warmupTime timeStep

global meanFrlSpeed sdFrlSpeed minFrlHeadway

global meanF21Speed sdF21Speed minF21Headway

global minSclHeadway

global goreLocation endPointScl sclLength sclRampCurveToGore distanceFromGore addonDist

tic

$\% \% \% \% \% \% \%$...... Setting up geometric parameters ....... \% \% \% \% \% \% \% \% $\% \% \%$

endPointScl $=$ [sclLength - sclRampCurveToGore]; \%last point for each SCL vehicle in the simulation

startPointFwl = goreLocation - distanceFromGore; \%first point for each FL vehicle to appear in simulation

endPointFwl = endPointScl + addonDist; \%last point for each FL vehicle in the simulation

f2lVolume $=$ round(frlVolume*laneRatio);

totalPNC $=$ zeros(sclVolume*nRuns, 1$) ; \%$ initialize vector to contain PNC results of all runs

nTotalSCLVeh $=0$;

iRun $=1 ; \% 1$ st run of the total of nRuns

while iRun $<=$ nRuns

fprintf('ไtRun \%d of \%d \n', iRun, nRuns)

$\%$ numbers of vehicles generated

nSimFrlVeh $=$ round $(1.2 *$ frlVolume*(1+warmupTime/3600) $) ; \%$ no. of simulated vehicles on FRL

nHVFrl $=$ round(nSimFrlVeh*pHVFrl);\% round the number of heavy vehicles

nSimSclVeh = sclVolume; \%no. of simulated vehicles on SCL 


$$
\begin{aligned}
& \text { if } \text { analMethod }==3 \\
& \text { nFrlInitial }=\text { nSimFrlVeh; }
\end{aligned}
$$

$\mathrm{nSimF} 2 \mathrm{lVeh}=\operatorname{round}(1.2 *$ f2lVolume $*(1+$ warmupTime $/ 3600)) ; \%$ no. of simulated vehicles on F2L

$$
\text { nHVF21 = round(nSimF2lVeh*pHVF21);\%round the number of heavy vehicles }
$$
end

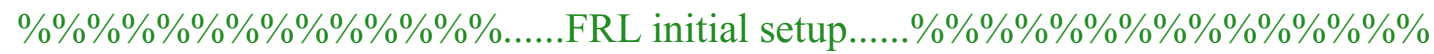

$\%$ call function to perform initial setup of FRL traffic: includes headway, speed, veh type (PC or HV)

\%calculate cummulative FRL headways: each vehicle will appear in the simulation when this cummulative headway is greater than or equal to the time elapsed

[frlSpeed, frlDecRate, frlHeadway, cumFrlHeadway, frlCritHeadway, ... frlVehLengths] = InitialSetupFwL_v5(frlVolume,meanFrlSpeed, ... sdFrlSpeed,minFrlHeadway,nSimFrlVeh, nHVFrl);

$\%$ initialize vectors for slow down or lane change requests for $\%$ cooperative merging for use in analMethod $=2 \& 3$

slowDownReq $=$ zeros $(1, \mathrm{nSimFrlVeh}) ; \%$ for use in analMethod $=2$

coopMergeTime $=$ zeros( $1, \mathrm{nSimFrlVeh}) ; \%$ for use in analMethod $=2$

$\%$ find max volume

$\mathrm{v} 1$ = find(cumFrlHeadway $>=$ warmupTime, 1$)$;

v2 $=$ find(cumFrlHeadway $<=(3600+$ warmupTime $), 1$,'last');

$\max \mathrm{Vol}=\mathrm{v} 2-\mathrm{v} 1+1$;

fprintf('Max volume on FRL is \%d veh/h \n', maxVol);

if analMethod $==3$

$\% \% \% \% \% \% \% \% \% \% \% \% \% \%$ \%.....F 2 L initial setup..... $\% \% \% \% \% \% \% \% \% \% \% \% \% \% \% \% \% \%$

$\%$ call function to perform initial setup of F2L traffic; similar to FRL

[f21Speed, f21DecRate, f21Headway, cumF21Headway, f21CritHeadway, ... f2lVehLengths] = InitialSetupFwL_v5(f2lVolume,meanF21Speed, ... sdF21Speed,minF21Headway,nSimF2lVeh, nHVF21);

$\%$ find max volume

$\mathrm{v} 1=$ find $($ cumF21Headway $>=$ warmupTime, 1$)$;

v2 = find(cumF2lHeadway $<=(3600+$ warmupTime $), 1$, 'last' $)$;

$\max \mathrm{Vol}=\mathrm{v} 2-\mathrm{v} 1+1$

fprintf('Max volume on F2L is \%d veh/h \n', maxVol);

firstSimF2LVeh $=1 ; \%$ first F2L vehicle in the simulation lastSimF2LVeh $=1 ; \%$ last F2L vehicle in the simulation 
f2lVehLocations $=$ ones( $1, \mathrm{nSimF} 2 \mathrm{lVeh}) *$ startPointFwl; \%initial locations of F2L vehicles

end

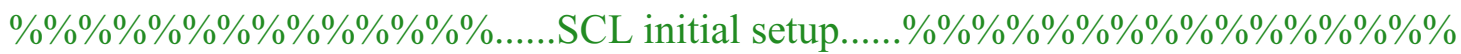

$\%$ call function to perform initial setup of SCL traffic: includes headway, gorespeed

\%cummulative FRL headways: each vehicle will appear in the simulation when

$\%$ this cummulative headway is greater than or equal to the time elapsed

[sclHeadway, cumSclHeadway, sclGoreSpeed, sclVehLengths] $=\ldots$

InitialSetupSCL_v12(warmupTime, sclVolume, nSimSclVeh, ...

gore85thSpeed, minSclHeadway);

if length(sclHeadway) $<$ nSimSclVeh

nSimSclVeh = length(sclHeadway);

fprintf('Only \%d SCL vehicles are generated in run \%d In', nSimSclVeh, iRun)

end

$\%$ find max volume

$\mathrm{v} 1=$ find $($ cumSclHeadway $>=$ warmupTime, 1$)$;

$\mathrm{v} 2=$ find $($ cumSclHeadway $<=(3600+$ warmupTime $), 1$, 'last' $)$;

$\max \mathrm{Vol}=\mathrm{v} 2-\mathrm{v} 1+1$;

fprintf('Max volume on SCL is \%d veh/h \n', maxVol);

$\% \% \% \% \% \% \% \% \% \% \% \% \% \% \ldots$.... Initializing Simulation ....... \% \% \% \% \% \% \% \% \% \% \% $\%$

simTime $=0 ; \%$ start time of simulation

firstSimFRLVeh $=1 ; \%$ first FRL vehicle in the simulation

lastSimFRLVeh $=1 ; \%$ last FRL vehicle in the simulation

frlVehLocations $=$ ones( $1, \mathrm{nSimFrlVeh}) *$ startPointFwl; \%initial locations of FRL vehicles

iSCLVeh $=0 ; \%$ start of SCL vehicle simulation

combinedPNC $=$ zeros $(1, \mathrm{nSimSclVeh})$;

$\% \quad$ if $\mathrm{iRun}==1$, iRun=iRun +1 ; continue, end

\%\%\%\%\%\%\%\%,......Start Simulation......\%\%\%\%\%\%\%\%

$\%$ Simulate vehicles as long as there are vehicles on the SCL and vehicles on

$\%$ the FRL are at the gore or upstream

while iSCLVeh<nSimSclVeh \&\& (lastSimFRLVeh<nSimFrlVeh \| frlVehLocations(lastSimFRLVeh) $<$ sclRampCurveToGore) 
simTime $=$ simTime + timeStep; $\%$ update simulation time

\%update vehicle locations, headways, and speeds of FRL vehicles in

$\%$ simulation; use a for loop to update the vehicles in sequence

rangeFrlVeh = firstSimFRLVeh:lastSimFRLVeh;

[vehLoc, headways, critHeadways, speeds, sdRequest $]=\ldots$

UpdateSpeedAndLocation_v8(minFrlHeadway, frlVehLocations(rangeFrlVeh), ... frlHeadway(rangeFrlVeh), frlCritHeadway(rangeFrlVeh), ... frlSpeed(rangeFrlVeh), frlDecRate(rangeFrlVeh), ...

frlVehLengths(rangeFrlVeh), slowDownReq(rangeFrlVeh));

frlVehLocations (rangeFrlVeh) $=$ vehLoc;

frlHeadway(rangeFrlVeh) = headways;

frlCritHeadway $($ rangeFrlVeh $)=$ critHeadways;

frlSpeed $($ rangeFrlVeh $)=$ speeds;

slowDownReq(rangeFrlVeh $)=$ sdRequest;

\%determine PNC for each SCL vehicle depending on the analysis method switch analMethod

case $\{0,1\}$

\%in this approach, release the SCL vehicle whose cumulative

$\%$ headway reached simulation time

if iSCLVeh $<$ nSimSclVeh \&\& simTime $>=$ cumSclHeadway (iSCLVeh+1)

iSCLVeh $=$ iSCLVeh +1 ;

$\%$ call function to calculate PNC total gap failure, PNC acceleration

\%failure and combined PNC

decRates $=$ frlDecRate $($ rangeFrlVeh $)$;

vehLen $=$ frlVehLengths (rangeFrlVeh);

sdRequest $=$ zeros $($ size $($ vehLen $))$;

cmMerge $=\operatorname{zeros}(\operatorname{size}(\operatorname{vehLen}))$;

[combinedPNC(iSCLVeh) $]=$ TotalPNC_v9 $($ simTime, iSCLVeh,.. sclGoreSpeed(iSCLVeh), sclVehLengths(iSCLVeh), cumSclHeadway(iSCLVeh), ... vehLoc, speeds, headways, critHeadways, decRates, vehLen, sdRequest, cmMerge);

end

case 2 
\%in this approach, release the SCL vehicle whose cumulative

\%headway reached simulation time, one FRL vehicle may be

\%asked to slow down to improve SCL vehicle merging

if iSCLVeh $<$ nSimSclVeh \&\& simTime $>=$ cumSclHeadway(iSCLVeh+1)

$\mathrm{iSCLVeh}=\mathrm{iSCLVeh}+1$;

\%call function to calculate PNC total gap failure, PNC acceleration

\%failure and combined PNC

decRates $=$ frlDecRate(rangeFrlVeh);

vehLen $=$ frlVehLengths(rangeFrlVeh);

sdRequest $=$ slowDownReq $($ rangeFrlVeh $)$;

cmMerge $=$ zeros $(\operatorname{size}($ vehLen $))$;

[combinedPNC(iSCLVeh), sdRequest $]=$ TotalPNC_v9(simTime, iSCLVeh, $\cdots$ sclGoreSpeed(iSCLVeh), sclVehLengths(iSCLVeh), cumSclHeadway(iSCLVeh), ... vehLoc, speeds, headways, critHeadways, decRates, vehLen, sdRequest, cmMerge);

\footnotetext{
slowDownReq(rangeFrlVeh $)=$ sdRequest; end
}

slowDownReq(frlVehLocations $>=$ endPointScl $)=0$;

case 3

\%in this approach, update the locations and speeds of

\%vehicles on 2nd FL

rangeF2lVeh $=$ firstSimF2LVeh:lastSimF2LVeh;

[vehLoc2, headways2, critHeadways 2 , speeds2] $=\ldots$

UpdateSpeedAndLocation_v8(minF21Headway,

f2lVehLocations(rangeF2lVeh), ...

f2lHeadway(rangeF2lVeh), f2lCritHeadway(rangeF2lVeh), ...

f21Speed(rangeF2lVeh), f2lDecRate(rangeF2lVeh), ...

f2lVehLengths(rangeF2lVeh));

f2lVehLocations $($ rangeF2lVeh $)=$ vehLoc2;

f2lHeadway $(($ rangeF2lVeh $))=$ headways 2 ;

f2lCritHeadway $($ (rangeF2lVeh $))=$ critHeadways2;

f21Speed $($ rangeF2lVeh $)=$ speeds 2 ; 
\%release the SCL vehicle whose cumulative headway reached

$\%$ simulation time, one FRL vehicle may be asked to change

\%lane to improve SCL vehicle merging

if iSCLVeh $<$ nSimSclVeh \&\& simTime $>=$ cumSclHeadway(iSCLVeh+1)

iSCLVeh $=$ iSCLVeh +1 ;

\%call function to calculate PNC total gap failure, PNC acceleration

\%failure and combined PNC

decRates $=$ frlDecRate $($ rangeFrlVeh $)$;

vehLen $=$ frlVehLengths(rangeFrlVeh);

sdRequest $=$ slowDownReq(rangeFrlVeh $)$;

cmMerge $=$ coopMergeTime $($ rangeFrlVeh $)$;

decRates2 = f21DecRate(rangeF2lVeh);

vehLen2 = f2lVehLengths(rangeF2lVeh);

iSCLVeh, ...

[combinedPNC(iSCLVeh), sdRequest, cmMerge] = TotalPNC_v9(simTime, cumSclHeadway(iSCLVeh), ...

sclGoreSpeed(iSCLVeh), sclVehLengths(iSCLVeh), cmMerge, ...

vehLoc, speeds, headways, critHeadways, decRates, vehLen, sdRequest,

vehLoc2, speeds2, headways2, critHeadways2, decRates2, vehLen2);

coopMergeTime $($ rangeFrlVeh $)=$ cmMerge;

end

\%check if FRL vehicle should shift to 2nd lane

lnChangVeh $=$ find $($ abs (coopMergeTime-simTime $)<$ timeStep/2,1);

if $\sim$ isempty(lnChangVeh)

$\%$ one vehicle will reach the time to change lane in the

$\%$ next time step

[frlVehLocations, frlHeadway, frlCritHeadway, frlSpeed, frlDecRate, frlVehLengths, slowDownReq, coopMergeTime, ...

f2lVehLocations, f2lHeadway, f2lCritHeadway, f21Speed, f2lDecRate, f2lVehLengths] $=$ LaneChange_v2 $(\operatorname{lnChangVeh}, \operatorname{minF} 21$ Headway,..

frlVehLocations, frlHeadway, frlCritHeadway, frlSpeed, frlDecRate, frlVehLengths, slowDownReq, coopMergeTime, ... f2lVehLengths);

f2lVehLocations, f2lHeadway, f2lCritHeadway, f21Speed, f21DecRate,

nSimFrlVeh $=$ nSimFrlVeh -1 ;

lastSimFRLVeh = lastSimFRLVeh - 1; 
end

\%update info of last F2L vehicle in simulation

if lastSimF2LVeh $<$ nSimF2lVeh \&\& simTime $>=$ cumF21Headway(lastSimF2LVeh+1)

lastSimF2LVeh = lastSimF2LVeh +1 ;

end

\%update info of 1st and F2L vehicle in simulation. check if

$\%$ firstSimF2LVeh reached the end of simulation. 1st vehicle is

$\%$ removed from the simulation only if there is at least one vehicle

$\%$ in the simulation area

if f2lVehLocations(firstSimF2LVeh) $>=$ endPointFwl \&\& firstSimF2LVeh $<$ lastSimF2LVeh end

end

\%update info of last FRL vehicle in simulation

if lastSimFRLVeh $<$ nSimFrlVeh \&\& simTime $>=$ cumFrlHeadway(lastSimFRLVeh+1)

lastSimFRLVeh $=$ lastSimFRLVeh +1 ;

end

\%update info of 1st and FRL vehicle in simulation. check if

$\%$ firstSimFRLVeh reached the end of simulation. 1st vehicle is

$\%$ removed from the simulation only if there is at least one vehicle

$\%$ in the simulation area

if frlVehLocations(firstSimFRLVeh) $>=$ endPointFwl \&\& firstSimFRLVeh $<$ lastSimFRLVeh

firstSimFRLVeh $=$ firstSimFRLVeh +1 ; end

end

if analMethod $==3$

fprintf('Total FRL vehicles shifting to 2nd lane is \%d 'n', (nFrlInitial-nSimFrlVeh)); end

totalPNC(nTotalSCLVeh+1:nTotalSCLVeh+nSimSclVeh) = combinedPNC';

nTotalSCLVeh $=$ nTotalSCLVeh + nSimSclVeh; 
clear frlSpeed frlDecRate frlHeadway cumFrlHeadway frlCritHeadway frlVehLengths switch analMethod

case 0

clear sclHeadway cumSclHeadway sclGoreSpeed sclVehLengths sclMergeSpeed sclMergeLength iMergeTime

case $\{1,2\}$

clear sclHeadway cumSclHeadway sclGoreSpeed sclVehLengths

case 3

clear sclHeadway cumSclHeadway sclGoreSpeed sclVehLengths

clear f21Speed f2lDecRate f2lHeadway cumF21Headway f2lCritHeadway

f2lVehLengths

end

$i$ Run $=i$ Run +1 ;

end

if nTotalSCLVeh $<$ length(totalPNC)

totalPNC(nTotalSCLVeh +1 :end $)=[]$;

end

meanPNC $=$ mean $($ totalPNC);

$\operatorname{sdPNC}=\operatorname{std}($ totalPNC);

fprintf('\nTotal number of simulated SCL vehicles is \%d \n', nTotalSCLVeh)

fprintf(' 'nMean and sd PNC are: \t \%8.6f and \%8.6f $\backslash n \backslash n '$, meanPNC, sdPNC)

toc 
function [headways, cumHeadway, goreSpeed, vehLengths] $=\ldots$

InitialSetupSCL_v12(initialHeadway,vehVolume,nVeh,gore85thSpeed,minSclHeadway)

global lengthPC

global multipleSD \%shared from the function initialSetupFRL

\%\%\%\%\%\%....... Initial Setup of SCL Vehicles......\%\%\%\%\%\%\%

\%assign normally distributed SCL speeds

\%first determine mean and SD of gore speeds based on 85th percentile

meanGoreSpeed $=-0.287+0.922 *$ gore 85 thSpeed; \% mean gore speed equation 4.2

sdGoreSpeed $=0.446+0.069 *$ gore 85 thSpeed; $\% \mathrm{SD}$ of gore speed equation 4.3

\%normally distributed random speeds

goreSpeed $=$ normrnd $($ meanGoreSpeed, sdGoreSpeed, $[1,2 * n V e h])$;

\%remove speeds that are higher or lower than mean plus or minus a specific multiple of $\mathrm{SD}$

maxGoreSpeed $=$ meanGoreSpeed + multipleSD $*$ sdGoreSpeed;

minGoreSpeed $=\max (0$, meanGoreSpeed - multipleSD*sdGoreSpeed $)$;

goreSpeed $($ goreSpeed $>$ maxGoreSpeed $)=[]$;

goreSpeed $($ goreSpeed $<$ minGoreSpeed $)=[]$;

goreSpeed $(\mathrm{nVeh}+1$ :end $)=[]$;

$\%$ setup SCL vehicle lengths considering all vehicles as passeneger cars

indexPassCarLength $=$ round $(($ length (lengthPC) -1$) *$ rand $(1, n V e h))+1 ; \%$ assign Passenger car length index considering uniform distribution

vehLengths $=$ lengthPC(indexPassCarLength);\%assign passenger car length index on different cars

$\%$ Random distribution of SCL vehicle headway

flowLambda $=$ vehVolume/3600; \%mean FRL headway is based on FRL veolume

headways $=(-1 /$ flowLambda $) * \log (\operatorname{rand}(1, \mathrm{nVeh})) ; \%$ headways generated correspond to number of simulated vehicles 


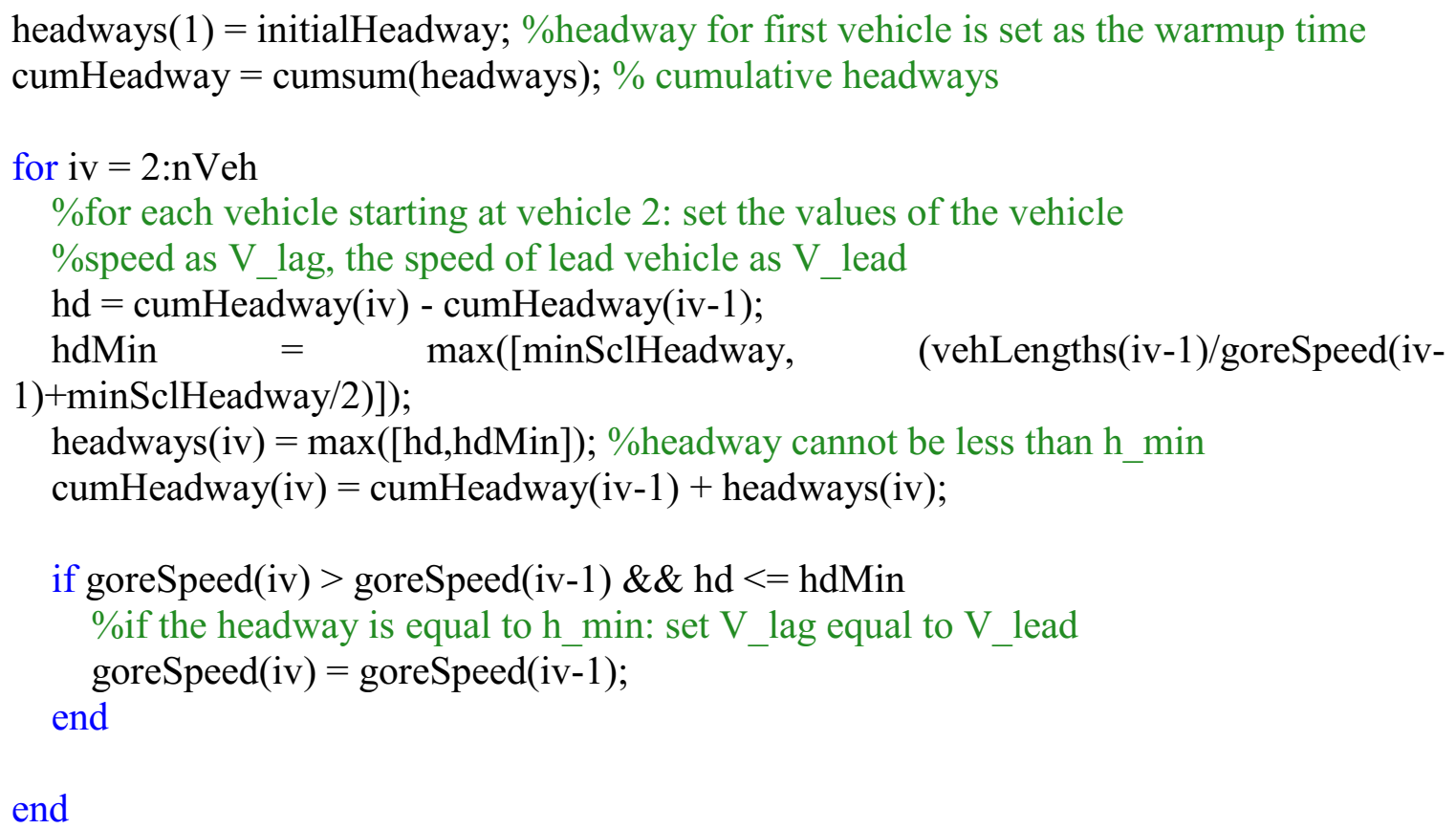


function [speeds, decelerations,headways,cumHeadway,critHeadway, vehLengths] ... $=$ InitialSetupFwL_v5(vehVolume,meanSpeed,sdSpeed,minHdVeh,nVeh,nHVeh)

global lengthPC lengthHV

global multipleSD \% to be shared with the function initialSetupSCL

$\% \% \% \% \% \%$.......Initial Setup of vehicles on a freeway lane ......\% $\% \% \% \% \% \%$

\section{\%Additional Inputs:}

multipleSD $=2.0 ; \%$ exclude extreme speed values that are mean plus or minus a specific multiple of SD

maxDecRate $=2 ; \%$ max deceleration rate for FRL vehicles if a lead vehicle is too slow decRange $=0.1 ; \%$ assumes that all vehicles are within + or- $10 \%$ of maxDecRate

\%assign normally distributed FRL speeds

for $\mathrm{i}=1$ : length(meanSpeed)

speeds $=$ normrnd(meanSpeed(i), sdSpeed, $[1,2 * n V e h])$;

$\%$ remove speeds that are higher or lower than mean plus or minus a specific multiple of $\mathrm{SD}$

maxSpeed $=$ meanSpeed(i) + multipleSD*sdSpeed;

$\operatorname{minSpeed}=\max (0$, meanSpeed $(\mathrm{i})-$ multipleSD $*$ sdSpeed $)$;

$\operatorname{speeds}(\operatorname{speeds}(:)>\max$ Speed $)=[]$;

speeds $(\operatorname{speeds}(:)<\operatorname{minSpeed})=[]$;

speeds $(\mathrm{nVeh}+1$ :end $)=[]$;

end

\%random distribution of FRL vehicle headway

flowLambda $=$ vehVolume $/ 3600 ; \%$ mean FRL headway is based on FRL veolume

headways $=(-1 /$ flowLambda $) * \log (\operatorname{rand}(1, \mathrm{nVeh})) ; \%$ headways generated correspond to number of simulated vehicles

$\%$ create unform distribution for deceleration rates for FRL vehicles

decelerations $=$ maxDecRate $*(1-$ decRange $)+2 *$ decRange*maxDecRate*rand $(1, n V e h)$;

\%setup vehicle lengths

\%first, assign random lengths assuming all vehicles are passenger cars

indexPassCarLength $=$ round $(($ length $($ lengthPC $)-1) *$ rand $(1, n V e h))+1 ; \%$ assign Passenger car length index considering uniform distribution

vehLengths = lengthPC(indexPassCarLength);\%assign passenger car length index on different cars 
\%then randomly assign nHVeh as heavy vehicles and change their lengths vehRandomOrder $=$ randperm $(\mathrm{nVeh}) ; \%$ randomly order the vehicles heavyVeh $=\operatorname{sort}(\operatorname{vehRandomOrder}(1: \mathrm{nHVeh})) ; \%$ first nHVeh in the randomly ordered vehicles are considered heavy vehicles

vehLengths(heavyVeh) = lengthHV;\%assign HV length

\%headway and speed adjustments:

$\% 1$ - any vehicle headway cannot be less than the min headway

$\%$ (front-to-front). It also cannot be less than the lead vehicle length

\%divided by its speed plus one half the min headway (back-to-front).

$\% 2$ - if a vehicle has a min headway, it cannot have a speed higher than its

$\%$ lead vehicle

\%use a for loop to ensure that the vehicle speeds are made in sequence and

$\%$ thus if 2 vehicles in succession need to change their speed, they both

$\%$ take the speed of the lead vehicle

$\% 3$ - every other lag vehicle that has greater speed than its lead vehicle will

$\%$ have to slow down once it reaches $\mathrm{h}$ min

$\%$ if the intitial headway is small enough, the speed of lag vehicle will

$\%$ have to be reduced in the initial setup

$\%$ this is checked that using the deceleration rate that needs to be adopted

\%by the lag vehicle to keep the headway equal to $\mathrm{h} \_$min

\%4- critHeadway is a vector of the critical headway values at which the fast

$\%$ lag vehicle will have to start deceleration

$\%$ initial values of critHeadway are zeros indicating that the default

$\%$ is that the lag vehicle does not decelerate

headways $(1)=0 ; \%$ headway for first vehicle is zero

cumHeadway $=$ cumsum(headways); \% cumulative headways

critHeadway $=$ zeros $($ size $($ speeds $))$;

for iv $=2: n V e h$

\%for each vehicle starting at vehicle 2: set the values of the vehicle

$\%$ speed as V_lag, the speed of lead vehicle as V_lead

$\%$ also set deceleration rate and headway

\%for min headway: take maximum of (1) hd_min and (2) length of lead

$\%$ vehicle divided by its speed plus $1 / 2$ hd_min

vLag $=\operatorname{speeds}(\mathrm{iv}) ; \mathrm{vLead}=\operatorname{speeds}(\mathrm{iv}-1)$;

decRate $=$ decelerations(iv);

hd = cumHeadway(iv) - cumHeadway(iv-1); 


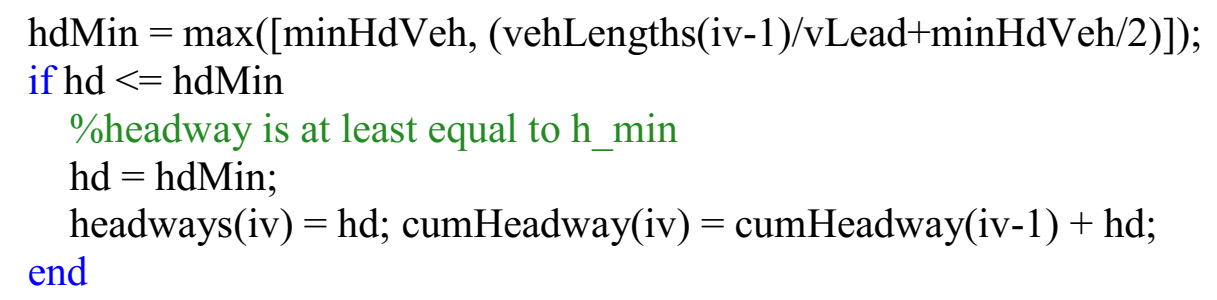

else

$\%$ the vehicle is faster than the lead vehicle but may not have

$\%$ to slow down at the initiation of simulation

$\%$ find the deceleration rate to maintain $\mathrm{h}$ _min when the lag

$\%$ vehicle reaches the speed of lead vehicle

\%formula: $d=0.5 *\left[\mathrm{~V} \_ \text {lag }-\mathrm{V} \_ \text {lead }\right]^{\wedge} 2 /\left[\mathrm{V} \_\right.$lag*h $-\mathrm{V} \_$lead $* \mathrm{~h} \_$min $]$

$\operatorname{decToVLead}=0.5 *\left((\overline{\mathrm{vLag}}-\overline{\mathrm{vLead}})^{\wedge} 2\right) /(\mathrm{v} \overline{\mathrm{Lag}} *$ hd $-\mathrm{vLead} *$ hdMin $) ; \%$ equation

if decToVLead $>$ decRate

$\%$ in this case, the initial speed of the lag vehicle is too

\%high; it needs to be adjusted at the beginning of the

$\%$ simulation

\%to find $\mathrm{V} \_$lag, solve a quadratic equation:

$\% V_{\_} l_{a g}^{\wedge} 2 \overline{+} b^{*} V_{-} l a g+c=0$

$\%$ solution gives 2 values of $V_{-}$lag: one higher than $\mathrm{V}$ lead

$\%$ (decelerating condition) and one lower than V_lead

$\%$ (accelerating ondition)

$\mathrm{b}=-2 *(\mathrm{vLead}+\mathrm{hd} *$ decRate $)$;

$\mathrm{c}=\operatorname{vLead} *(\mathrm{vLead}+2 *$ hdMin $*$ decRate $)$;

$\mathrm{xx}=\mathrm{b}^{\wedge} 2-4^{*} \mathrm{c}$;

if $\mathrm{xx}<0$, error('error (1) adjusting speed of FRL veh \% $\mathrm{d}$ ', iv); end

$\operatorname{vLagAdj}=(-\mathrm{b}+\operatorname{sqrt}(\mathrm{xx})) / 2 ; \%$ equation 5.5 ; solution of quadratic equation but selecting the higher solution

if $v$ LagAdj $<$ vLead $\|$ vLagAdj $>$ vLag

end

error('error (2) adjusting speed of FRL veh \%d', iv)

speeds $(\mathrm{iv})=$ vLagAdj;

critHeadway(iv) = hd; \%critHeadway is the current headway

else

\%find new vehicles that are faster than the lead but do not 
$\%$ have to decelerate from the beginning of simulation;

$\%$ critical headway for those vehicles is the headway at which

$\%$ deceleration should start

$\%$ equation: $h \_c r i t=\left[0.5 *\left(V \_l a g-V \_l e a d\right) \wedge 2 / d \_m a x+V \_l e a d * h \_m i n\right] / d \_m a x$

critHeadway(iv) $=\max ([\mathrm{hdMin}+(\mathrm{vLag}-\mathrm{vLead}) / \mathrm{decRate}, \quad((\mathrm{vLag}-$ vLead $\left.)^{\wedge} 2\right) /(2 *$ decRate $)+$ vLead $*$ hdMin $) / v$ Lag] $)$;

\author{
end \\ end
}

end

end 
function [vehLoc, headways, critHeadways, speeds, slowRequest] $=\ldots$ UpdateSpeedAndLocation_v8(minHdVeh, vehLoc, headways, critHeadways, ... speeds, decRates, vehLen, slowRequest)

global timeStep maxReqGap

$\% \% \% \%$...Update locations and speeds of vehicles on freeway lane...\%\%\%

$\mathrm{nVeh}=$ length(vehLoc);

if nargin $==7$

slowRequest $=$ zeros $(1, \mathrm{nVeh})$;

end

\%for first vehicle in the simulation, adjust speed only because there is no

$\%$ lead vehicle and headway is not updated

$\operatorname{vehLoc}(1)=\operatorname{vehLoc}(1)+\operatorname{speeds}(1) *$ timeStep;

$\%$ for all other vehicles, the update requires speed location of lead

$\%$ vehicle and the headway is updated

for iv $=2: \mathrm{nVeh}$

if slowRequest(iv) $==0 \|$ (headways(iv) $>=\operatorname{maxReqGap}+$ vehLen(iv-1)/speeds(iv))... $\|$ (speeds(iv) $>$ speeds(iv-1) $\& \&$ headways(iv) $<=$ critHeadways(iv))

\%regular update of the vehicle speed and location

if speeds(iv) $<=$ speeds(iv-1)

$\%$ vehicle is slower than its lead vehicle; headway is increasing

vehLoc(iv) $=\operatorname{vehLoc(iv)~}+$ speeds(iv)*timeStep;

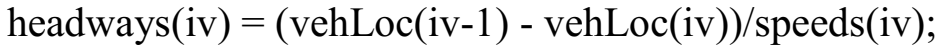

elseif headways(iv) $>$ critHeadways(iv)

$\%$ vehicle is slower than its lead vehicle but headway is greater

$\%$ than the critical headway

vehLoc(iv) $=\operatorname{vehLoc(iv)~}+$ speeds(iv)*timeStep;

headways(iv) $=(\operatorname{vehLoc}(i v-1)-\operatorname{vehLoc}(i v)) /$ speeds(iv);

hdMin $=\max ([\operatorname{minHdVeh},(\operatorname{vehLen}(\mathrm{iv}-1) / \operatorname{speeds}(\mathrm{iv}-1)+\operatorname{minHdVeh} / 2)])$;

\%if the diff between original headway and critical headway is too

$\%$ small, the new headway may drop below critical headway

if headways(iv) $<=$ critHeadways(iv)

headways(iv) $=$ critHeadways(iv); 


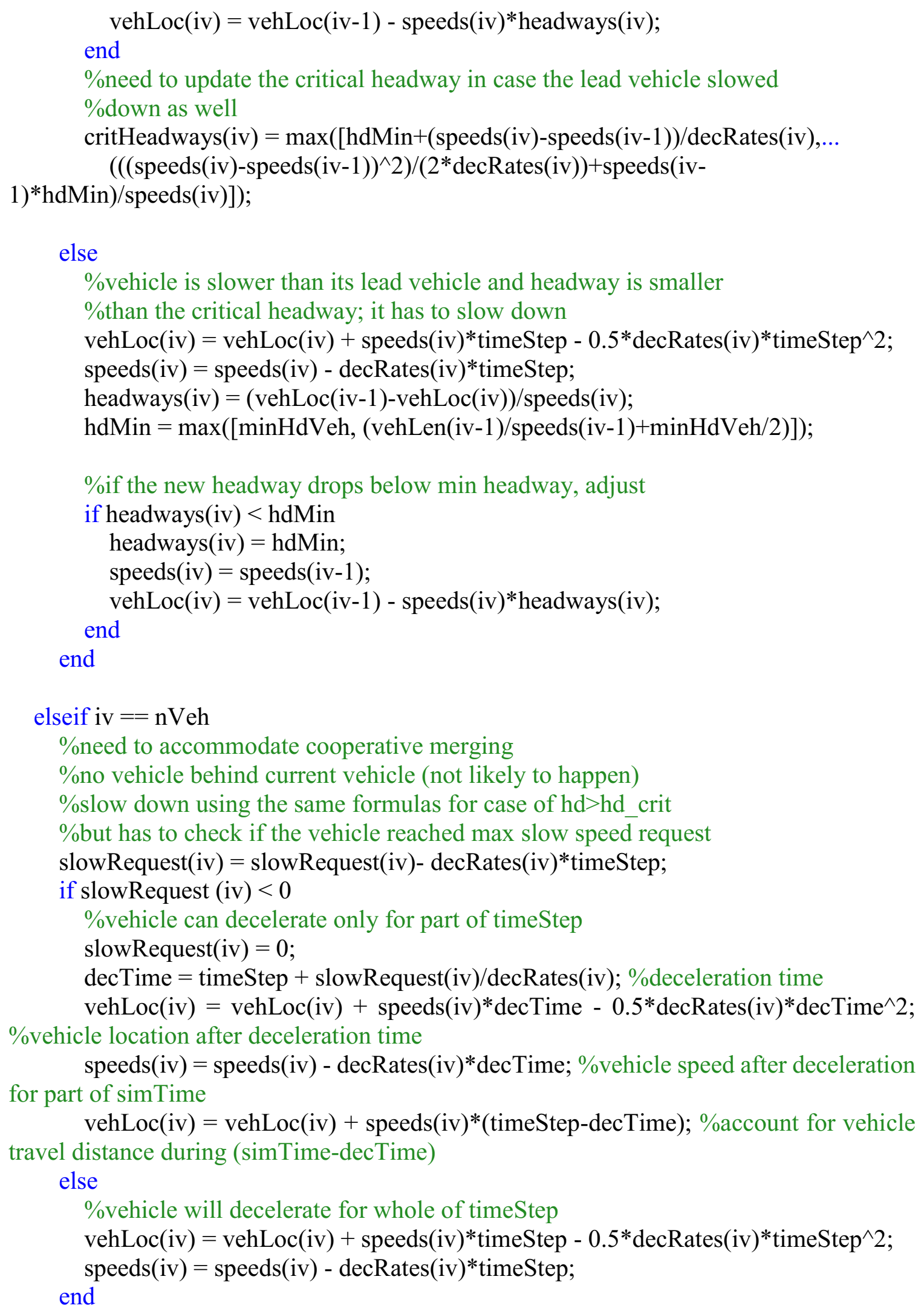


headways(iv) $=(\operatorname{vehLoc}(i v-1)-\operatorname{vehLoc}(i v)) /$ speeds(iv);

\%check headway in case the lead vehicle was decelerating at a

$\%$ higher rate

hdMin $=\max ([\operatorname{minHdVeh},(\operatorname{vehLen}(\mathrm{iv}-1) / \operatorname{speeds}(\mathrm{iv}-1)+\operatorname{minHdVeh} / 2)])$;

$\%$ if the new headway drops below min headway, adjust

if headways(iv) $<$ hdMin

headways(iv) $=$ hdMin;

speeds $($ iv $)=\operatorname{speeds}($ iv-1 $)$;

vehLoc(iv) $=\operatorname{vehLoc}(i v-1)-\operatorname{speeds}(i v) * h e a d w a y s(i v)$;

end

else

\%need to accommodate cooperative merging while accounting for

$\%$ impact on lag vehicle

\%calculate current headway and min headway for lag vehicle

hdLag $=(\operatorname{vehLoc}(i v)-\operatorname{vehLoc}(i v+1)) /$ speeds $(i v+1)$;

hdLagMin $=\max ([\operatorname{minHdVeh},(\operatorname{vehLen}(\mathrm{iv}) / \operatorname{speeds}(\mathrm{iv})+\operatorname{minHdVeh} / 2)])$;

if $\quad$ speeds(iv) $>$ speeds(iv +1$) \quad \| \quad$ (hdLag $>2 *$ hdLagMin $\quad \& \&$

headways $(\mathrm{iv}+1)>$ critHeadways $(\mathrm{iv}+1)$ )

$\%$ in this case, vehicle can slow down for cooperative merging

slowRequest(iv) = slowRequest(iv)- decRates(iv)*timeStep;

if slowRequest (iv) $<0$

slowRequest(iv) $=0$;

decTime $=$ timeStep + slowRequest(iv)/decRates(iv);

vehLoc(iv) $=\operatorname{vehLoc}($ iv $)+\operatorname{speeds}(\text { iv })^{*} \operatorname{dec}$ Time $-0.5 * \operatorname{decRates}(\mathrm{iv}) * \operatorname{dec} \operatorname{Time}^{\wedge} 2$;

speeds(iv) $=$ speeds(iv) - decRates(iv)*decTime;

vehLoc(iv) $=\operatorname{vehLoc}(\mathrm{iv})+\operatorname{speeds}(\mathrm{iv}) *($ timeStep-decTime $)$;

else

$\operatorname{vehLoc}($ iv $)=\operatorname{vehLoc}(i v)+\operatorname{speeds}($ iv $) *$ timeStep $-0.5 *$ decRates $(i v) *$ timeStep $^{\wedge} 2$

speeds(iv) $=$ speeds(iv) - decRates(iv)*timeStep; end

else

$\%$ vehicle's speed is less than or equal to the lag vehicle and

$\%$ lag vehicle headway is less than $2 \mathrm{~h}$ _min; vehicle cannot slow down

vehLoc(iv) $=\operatorname{vehLoc(iv)~}+$ speeds(iv)*timeStep;

end

headways(iv) $=(\operatorname{vehLoc}(i v-1)-\operatorname{vehLoc}(i v)) /$ speeds(iv);

$\%$ check headway in case the lead vehicle was decelerating at a

$\%$ higher rate

hdMin $=\max ([\operatorname{minHdVeh},(\operatorname{vehLen}(\mathrm{iv}-1) / \operatorname{speeds}(\mathrm{iv}-1)+\operatorname{minHdVeh} / 2)])$; 
\%if the new headway drops below min headway, adjust

if headways(iv) $<$ hdMin

headways(iv) $=$ hdMin;

speeds $(\mathrm{iv})=\operatorname{speeds}(\mathrm{iv}-1)$;

vehLoc(iv) $=\operatorname{vehLoc}(i v-1)-\operatorname{speeds}(i v) * h e a d w a y s(i v)$;

end

\%need to update the critical headway in case the lead vehicle slowed

$\%$ down as well and also update crittical headway for lag

$\%$ vehicle

critHeadways $(\mathrm{iv})=\max ([\mathrm{hdMin}+(\operatorname{speeds}(\mathrm{iv})-\operatorname{speeds}(\mathrm{iv}-1)) / \operatorname{decRates}(\mathrm{iv}), \ldots$

$\left(\left((\text { speeds(iv)-speeds(iv-1) })^{\wedge} 2\right) /(2 * \operatorname{decRates}(\mathrm{iv}))+\right.$ speeds(iv-

1)*hdMin)/speeds(iv)]);

end

\%finished updating vehicle iv; move to next vehicle

end 
function [availTotGaps, locGapBegin, gapSpeeds] =

TotalGap_v8(vehLoc,speeds,vehLen,laneCode)

global meanFrlSpeed goreLocation endPointScl distanceFromGore

if nargin $<4$

$\%$ default lane is FRL; code $=1$

laneCode $=1$;

end

$\% \% \% \% \% \%$..........Calculating available space gaps......\%\%\%\%\%\%

\%output:

$\%$ the total space gaps

\%location of the beginning of each gap

$\%$ speed of lag vehicle for each gap (if there is a gap with no lag vehicle,

$\%$ the mean FRL speed is used)

$\%$ gaps are found between loc1 \& endPointScl

switch laneCode

case 1

$\%$ FRL; gaps are found in the SCL area: loc 1 = goreLocation

$\operatorname{loc} 1$ = goreLocation;

case 2

$\% 2$ nd lane; gaps are found from beginning of simulation area to end

$\%$ of SCL

end

loc 1 = goreLocation - distanceFromGore;

i1 = find(vehLoc $>=$ endPointScl,1,'last'); \%last vehicle downstream SCL

i2 = find (vehLoc $<=1$ loc 1,1); \%first vehicle upstream SCL

iGaps $=\mathrm{i} 1: \mathrm{i} 2$;

if (isempty(i1) \&\& isempty(i2)) $\|$ (isempty(i1) \&\& i2 ==1) $\|$ (isempty(i2) \&\& i1 == length(vehLoc))

$\%$ this is a case where both the last vehicle downstream SCL and

$\%$ first vehicle upstream SCL are beyond the simulation limits or there

$\%$ are no FRL vehicles in the SCL area

\%may happen if FRL volume is very low

iGaps $=[1]$

availTotGaps $(1)=$ endPointScl-loc1;

gapSpeeds $=[$ meanFrlSpeed $]$; 


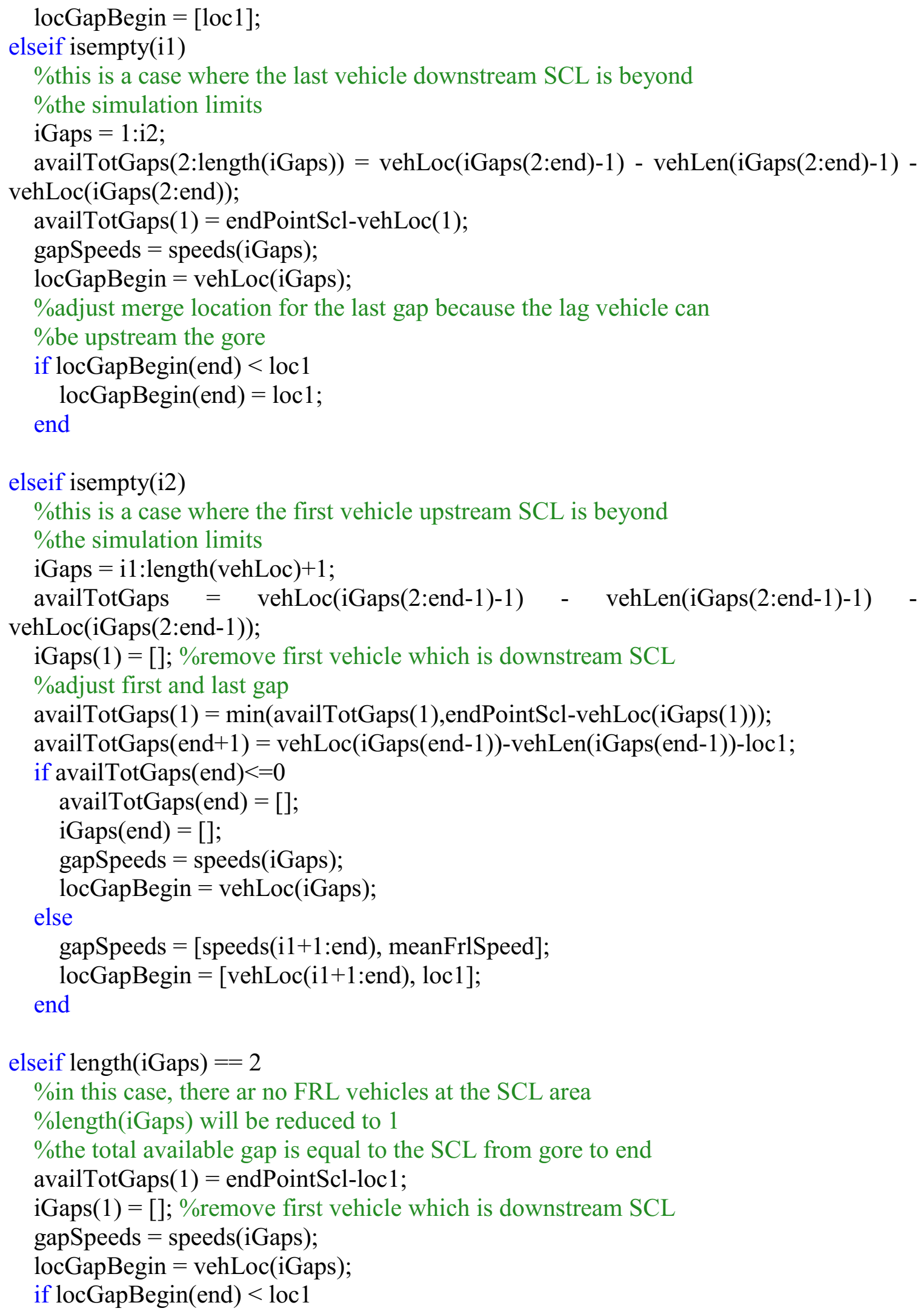




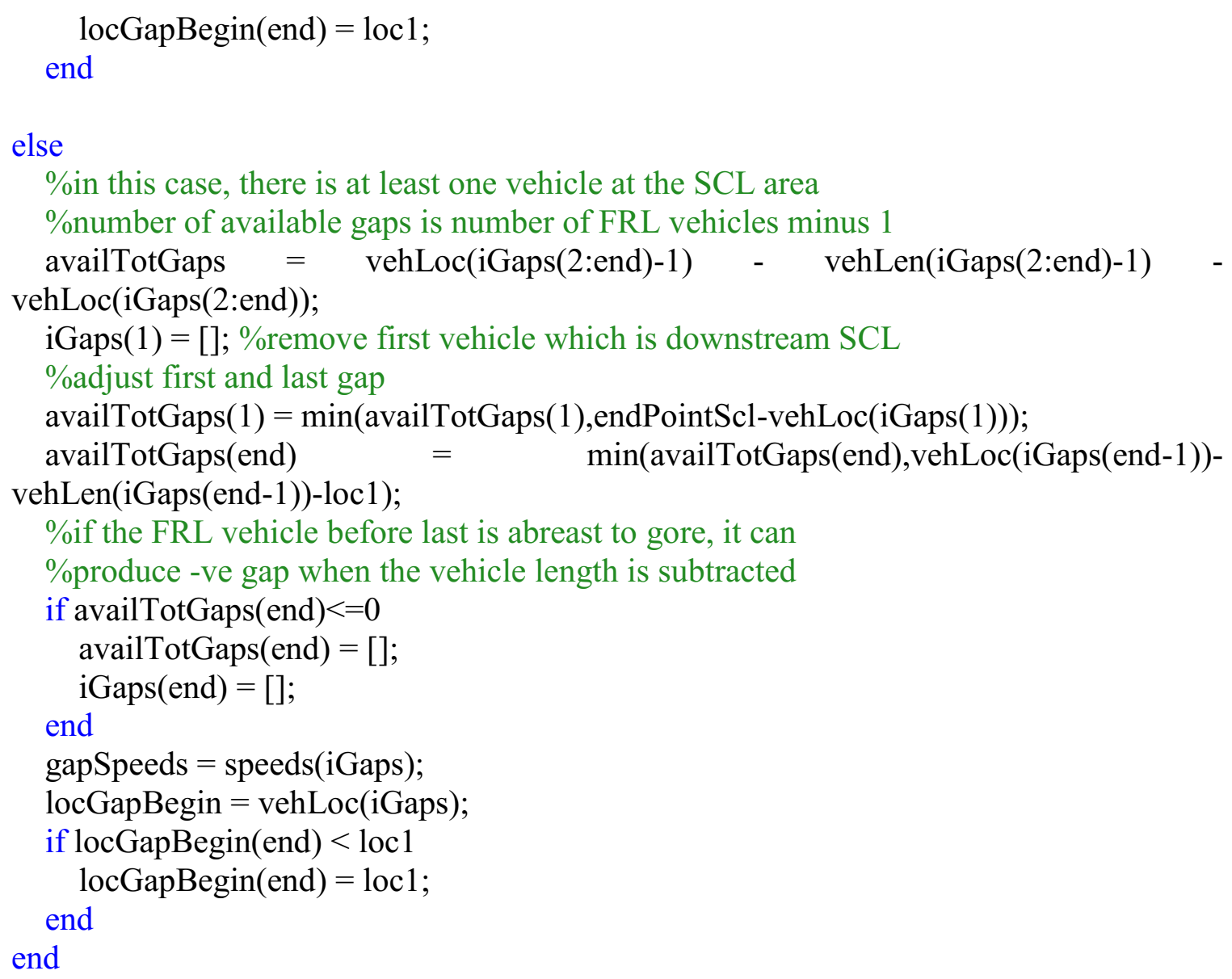


function [coopMerge, lagVeh] = DecideCoopMerge_v3(meanLoc2Merge, vehLoc, ... speeds, headways, vehLen)

global maxReqGap

global goreLocation endPointScl distanceFromGore

global analMethod

global meanFrlSpeed minFrlHeadway

$\% \% \% \%$...Decide whether cooperative merge is to be attempted... $\% \% \% \%$

simBegin $=$ goreLocation - distanceFromGore; \%first point in simulation

$\%$ find the lead FRL vehicle (first ahead of the SCL vehicle) and lag vehicle

leadVeh $=$ find(vehLoc $>$ meanLoc2Merge, 1 ,'last');

if isempty(leadVeh)

$\%$ the lead vehicle is downstream the end of simulation

$\%$ no lead vehicle; lag vehicle is vehicle \# 1

$\operatorname{lagVeh}=1$;

timeGapFrl $=($ endPointScl $-\operatorname{vehLoc}(\operatorname{lagVeh})) /$ speeds $(\operatorname{lagVeh})$;

elseif (vehLoc(leadVeh)-vehLen(leadVeh)) $>=$ endPointScl \&\& leadVeh $<$ length(vehLoc)

$\%$ lead vehicle is downstream end of SCL but still within

$\%$ simulation limits; there is a lag vehicle in the SCL area

lagVeh = leadVeh +1 ;

timeGapFrl = (vehLoc(leadVeh) $-\operatorname{vehLoc}(\operatorname{lagVeh})) /$ speeds $($ lagVeh $)$;

elseif (vehLoc(leadVeh)-vehLen(leadVeh)) >= endPointScl

$\%$ lead vehicle is downstream end of SCL but still within

$\%$ simulation limits; there is no lag vehicle in the SCL area

$\operatorname{lagVeh}=[]$

timeGapFrl $=(\operatorname{vehLoc}($ leadVeh $)-\operatorname{vehLen}($ leadVeh $)-\operatorname{simBegin}) /$ meanFrlSpeed;

elseif leadVeh $<$ length(vehLoc)

$\%$ at least end of the lead vehicle is within the SCL length

lagVeh = leadVeh +1 ;

timeGapFrl = (vehLoc(leadVeh) - vehLoc(lagVeh) $) /$ speeds $(\operatorname{lagVeh})$;

else

$\%$ no lag vehicle within the data

$\operatorname{lagVeh}=[]$

timeGapFrl $=(\operatorname{vehLoc}($ leadVeh $)-\operatorname{vehLen}($ leadVeh $)-\operatorname{simBegin}) /$ meanFrlSpeed; end

switch analMethod 
case 2

$\%$ find out whether the lag vehicle need and can decelerate to

$\%$ accommodate cooperative merging

if timeGapFrl >= maxReqGap $\|$ isempty(lagVeh)

$\%$ available time gap at mean merge is accepted by all drivers

$\%$ or there is no lag vehicle; no need to request speed adjustments

coopMerge $=0$;

elseif lagVeh $==$ length(vehLoc)

\%lag vehicle is the last vehicle on the FRL; it can decelerate

$\%$ freely

else

coopMerge $=1$;

$\%$ find actual and min headway between the lag vehicle and the

$\%$ vehicle after it

hdLag $=$ headways $(\operatorname{lagVeh}+1)$;

hdMin =

(vehLen(lagVeh)/speeds(lagVeh+1)+minFrlHeadway/2)]);

if hdLag $<=2 *$ hdMin

$\%$ vehicle will slow down only if the vehicle after has at

$\%$ least twice the min headway and has not reached

$\%$ critical headway

coopMerge $=0$;

else

coopMerge $=1$;

end

end

case 3

$\%$ find out whether the lag vehicle need and can change lane to

\%accommodate cooperative merging

if timeGapFrl >= maxReqGap $\|$ isempty(lagVeh)

\%available time gap at mean merge is accepted by all drivers

$\%$ or there is no lag vehicle; no need to request speed adjustments

coopMerge $=0$

else

coopMerge $=1$

end

end 
function [vehLocL1, hdwayL1, critHdL1, speedL1, decRateL1, vehLenL1, sdRequest, cmTime, ...

vehLocL2, hdwayL2, critHdL2, speedL2, decRateL2, vehLenL2] = LaneChange_v2(iv1, minHdVeh, ...

vehLocL1, hdwayL1, critHdL1, speedL1, decRateL1, vehLenL1, sdRequest, cmTime, ...

vehLocL2, hdwayL2, critHdL2, speedL2, decRateL2, vehLenL2)

$\% \% \% \%$...Find location, speed \& headway of a vehicle changing lane... $\% \% \% \%$

$\%$ Original lane: L1

$\%$ New lane: L2

vehLocOld $=$ vehLocL1(iv1);

vehLen $=$ vehLenL1(iv1);

decRate $=$ decRateL1(iv1);

$\%$ find index of lane changing vehicle in new lane

iv2 = find (vehLocL2 $<$ vehLocOld,1);

\%estimate min headway to lag and lead vehicles

if isempty(iv2)

$\%$ there is no lag vehicl in the 2nd lane

hdMinLead $=\max ([\operatorname{minHdVeh},(\operatorname{vehLenL} 2($ end $) / \operatorname{speedL2}($ end $)+\operatorname{minHdVeh} / 2)])$;

$\%$ find new veh location: set it as the veh location in the original lane but

$\%$ cannot be less than the old veh location and hd_min from the lead vehicle

maxNewVehLoc $=\operatorname{vehLocL2}($ end $)-$ hdMinLead $*$ speedL1(iv1);

newVehLoc $=\min ($ vehLocOld, $\operatorname{maxNewVehLoc})$;

$\%$ veh speed in the new lane does not change

newVehSpeed $=$ speedL1(iv1);

newHd = (vehLocL2(end) - newVehLoc)/newVehSpeed;

$\%$ calculate critical headways for lead vehicle

if newVehSpeed $>$ speedL2(end)

vLead $=$ speedL2(end);

$\mathrm{vLag}=$ newVehSpeed;

hdMin = hdMinLead; 


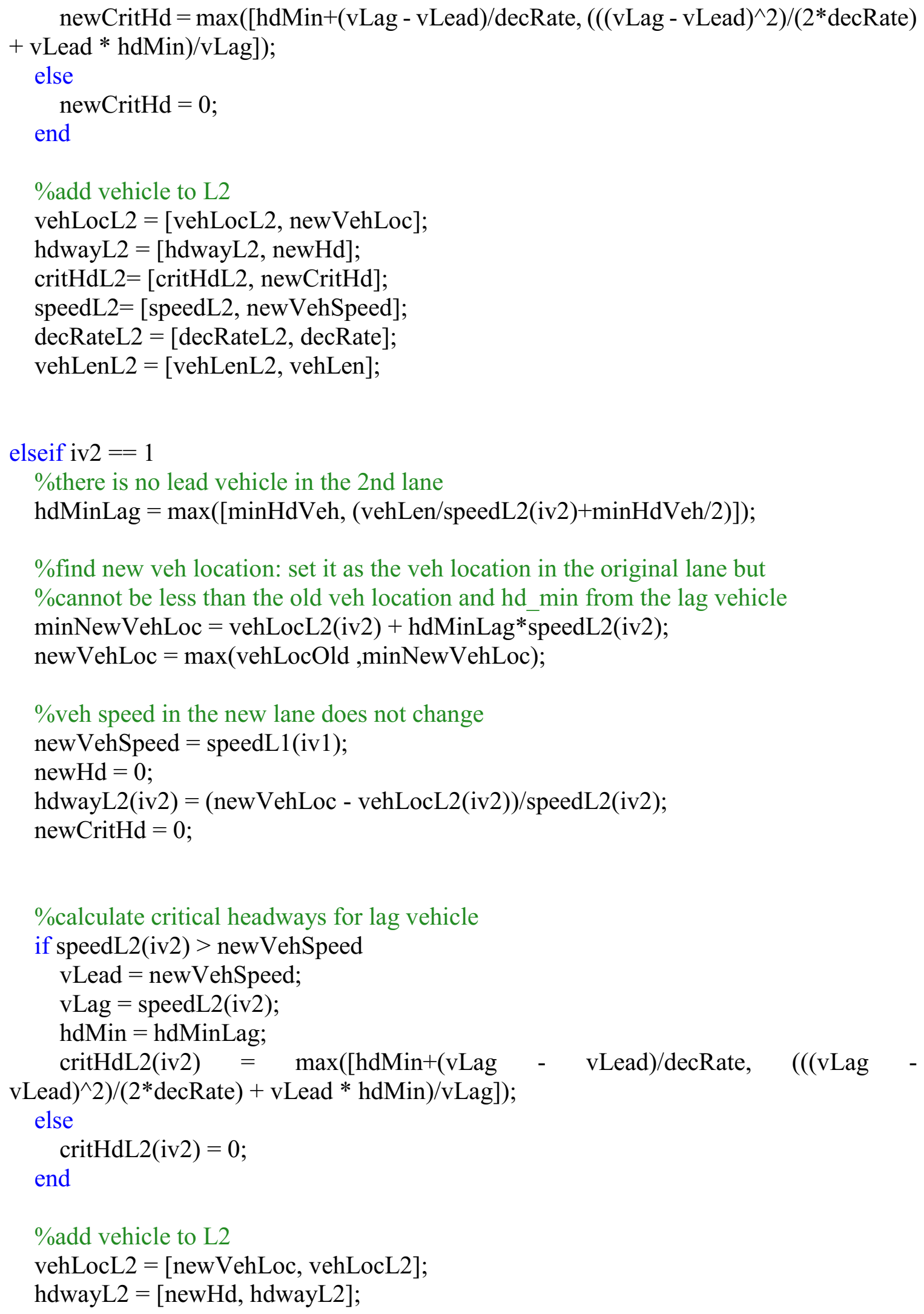


critHdL2 $=[$ newCritHd, critHdL2];

speedL2 $=$ [newVehSpeed, speedL2];

decRateL2 = [decRate, decRateL2];

vehLenL2 = [vehLen, vehLenL2];

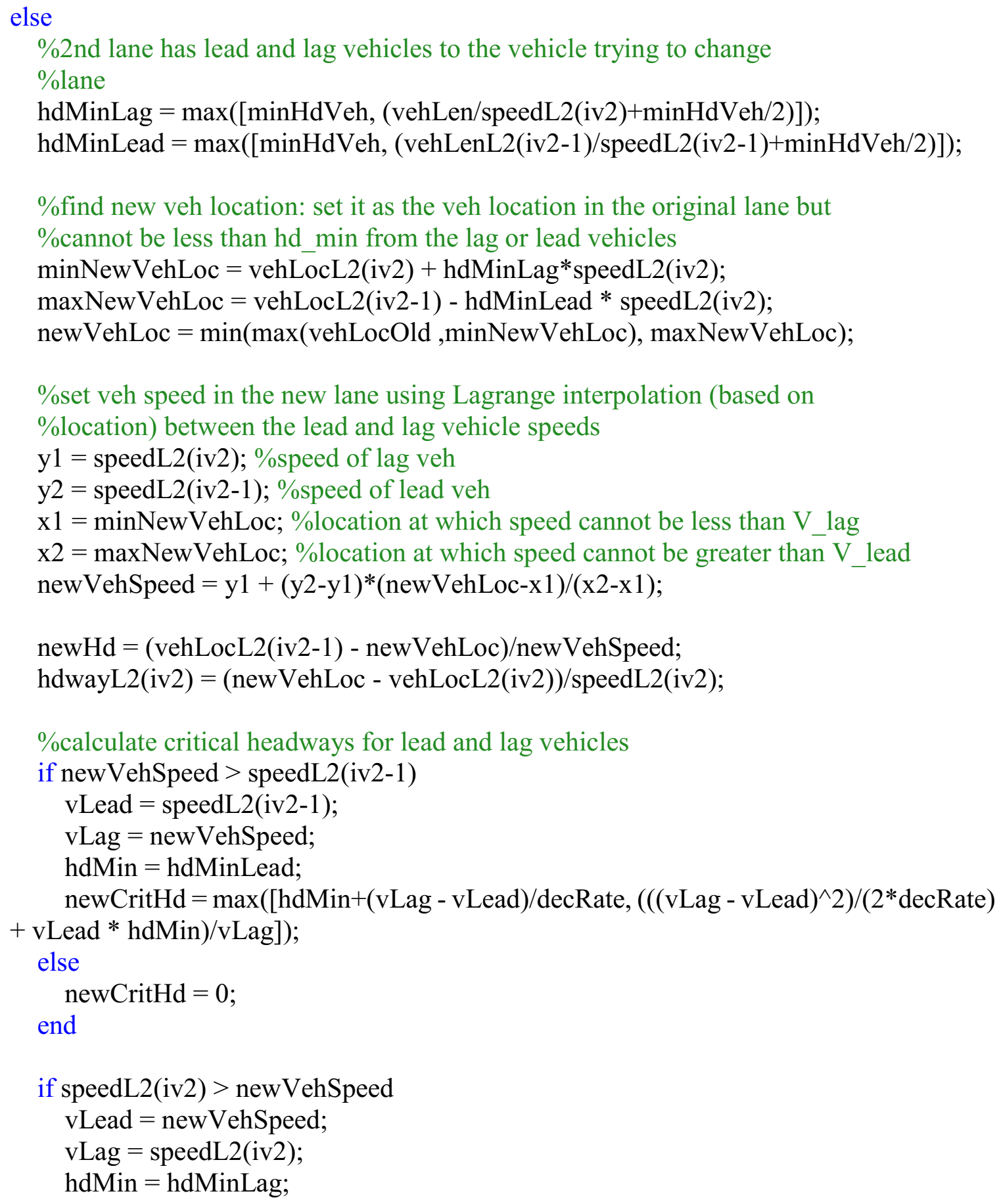




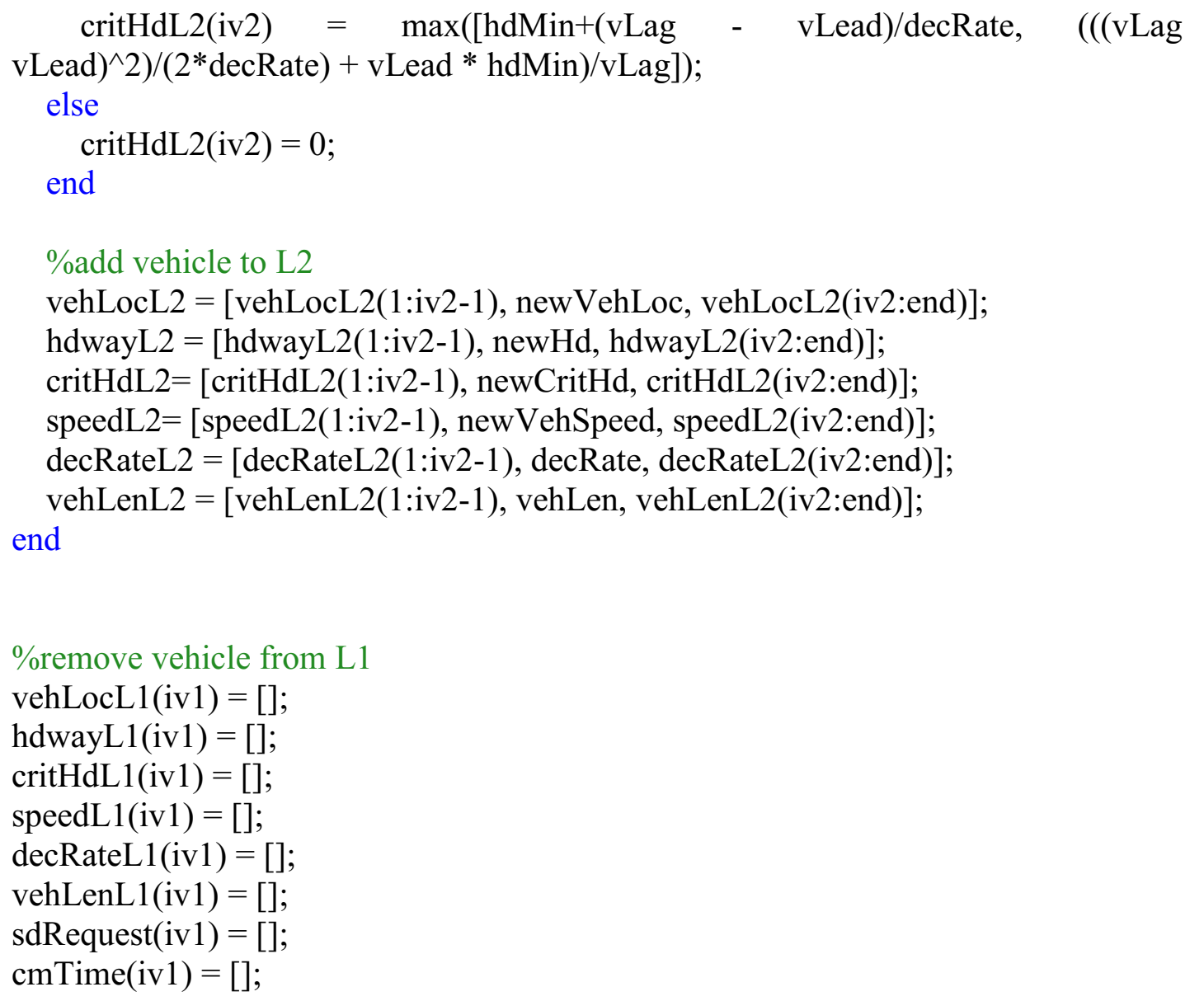




\section{TotalPNC_v9.m}

function [finalCombinedPNC, sdRequest, cmMergeRin] = TotalPNC_v9(simTime, iSCLVeh,... goreSpeed, sclVehLen, releaseTime, vehLocR, speedsR, headwaysR, ... critHeadwaysR, decRatesR, vehLenR, sdRequest, cmMerge, vehLoc2, speeds2, headways $2, \ldots$

critHeadways2, decRates2, vehLen2)

global timeStep maxReqGap

global sclLength sclRampCurveToGore

global analMethod

global minFrlHeadway

global minF2lHeadway

global sdAcceptTotGap \% shared to m0TotalPNC

$\% \% \% \% \%$....Calculate Min Combine PNC for a specific SCL Vehicle.... $\% \% \% \% \% \%$

$\% \% \% \% \% \% \% \% \% \% \% \%$...All Analysis Methods (1, 2 \& 3)...\%\%\%\%\%\%\%\%\%\%\%\%

$\%$ Method 1: search for best location and time for SCL vehicle to merge based

$\%$ on min combined PNC for acceleration and gap acceptance

\%Method 2: similar to Method 1 but FRL one vehicle may be required to slow

\%down to accommodate the SCL vehicle merging (cooperative merging)

\%Method 3: similar to Method 1 but FRL one vehicle may be required to

\%change lane to 2 nd fwy lane to accommodate the SCL vehicle merging (cooperative merging)

$\%$ Additional input:

ratioLagGap $=0.4 ; \%$ proportion of the total gap between SCL vehicle and lag FRL vehicle $\max$ SlowReq $=0.1 ; \% \max$ speed to be reduced when there is a slow request

acceptMergeGapLn2 =6; \% the gap size in 2nd lane to be accepted by FRL vehicle to shift lane

\%determine mean merge speed, sd of accepted gap, and meand \& sd acceleration

$\%$ only parameters that do not depend on merge location

$\%$ mean accepted gap depends on merge locations and to be determined later

if sclVehLen $>5.207$

vehType $=1$;

else

vehType $=0$

end

\%Initialize values that do not change for a specific SCL vehicle 
\%estimate mean merge speed from a driver-level regression model

\%in all methods, this is the mean merge speed for a vehicle with this

\%gore speed

meanMergeSpeed $=17.42+0.014 *\left(\right.$ goreSpeed $\left.^{\wedge} 2\right)-0.95 *$ vehType; \%equation 4.4

if meanMergeSpeed $<$ goreSpeed

meanMergeSpeed = goreSpeed;

end

sdAcceptTotGap $=0.963 ; \%$ Standard deviation of total gap $=0.963$

meanAccRate $=1.53-0.05 *$ goreSpeed- $0.19 *$ vehType; \%equation 4.5 ; mean acceleration rate from regression models

sdAccRate $=0.288 ; \%$ standard deviation of acceleration rate from regression models

\%initial and final time for possible merging by the SCL vehicle

\%assumes SCL vehicle travels at constant speed without acceleration

sclVehT1 $=$ releaseTime + sclRampCurveToGore/goreSpeed;

sclVehT2 $=$ releaseTime + sclLength/goreSpeed;

if analMethod $>=2$

$\%$ for cooperative merging, target the gap to be reached by mean acc rate and

$\%$ mean merge speed

\%estimate time for the SCL vehicle to reach acceptable merge speed

meanTime2Merge $=$ sclVehT1 $+($ meanMergeSpeed - goreSpeed $) /$ meanAccRate;

meanLoc2Merge $=\left(\right.$ meanMergeSpeed $^{\wedge} 2-$ goreSpeed $\left.^{\wedge} 2\right) /(2 *$ meanAccRate $) \quad-$ sclRampCurveToGore;

end

if analMethod $<=1$

analysisRun $=1 ; \%$ analysis will be run only once

else

analysisRun $=2 ; \%$ analysis may be run twice

$\%$ store original (initial) values of RL \& 2 ndLane speed variables that will

$\%$ change in calculating PNC for re-use in analMethod $2 \& 3$

vehLocRin = vehLocR;

headwaysRin = headwaysR;

critHeadwaysRin $=$ critHeadwaysR;

speedsRin = speedsR;

sdRequestRin $=$ sdRequest;

cmMergeRin $=$ cmMerge;

$\operatorname{lagVeh}=[]$ 


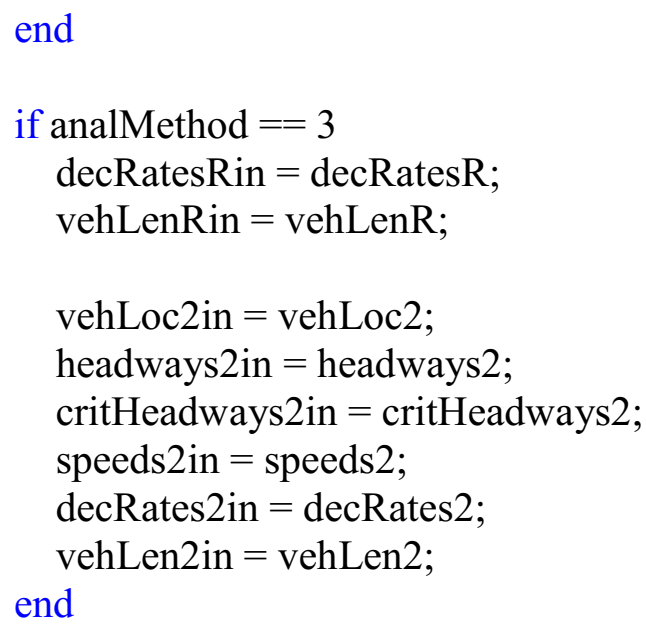


[vehLoc2, headways2, critHeadways 2 , speeds2] $=\ldots$

UpdateSpeedAndLocation_v8(minF2lHeadway, vehLoc2, headways2, ... critHeadways2, speeds2, decRates2, vehLen2);

end

end

$\%$ for analMethod $2 \& 3$, find the time closest to meanTime2Merge

if analysisRun $>1 \& \&$ abs(analTime - meanTime2Merge) $<=$ timeStep/2

[coopMerge, lagVeh] = DecideCoopMerge_v3(meanLoc2Merge, vehLocR,... speedsR, headwaysR, vehLenR);

lagVehRin $=$ vehIdx(lagVeh); \%adjust index of lag veh in case a vehicle has changed lane

if coopMerge $==1 \& \&$ cmMerge $($ lagVeh $)>0$

coopMerge $=0 ; \%$ lag vehilce will already change lane

end

end

\%for second run in analMethod 3, check if the lag vehicle can

$\%$ shift to 2nd lane

if analMethod $==3 \& \&$ analysisRun $==1 \& \&$ coopMerge $==1$

$\%$ call function to calculate the gaps in 2nd lane at SCL area and determine

$\%$ the corresponding time gaps

[availTotGaps2, locGapBegin2,

gapSpeeds2]

TotalGap_v8(vehLoc2,speeds2,vehLen2,2);

availTotTimeGaps $=$ availTotGaps2./gapSpeeds2;

\%determine the 2nd lane's gap corresponding to FRL lag vehicle

iGap $=$ find $($ vehLocR $(\operatorname{lagVeh})>=$ locGapBegin 2,1$)$;

\%the FRL lag vehicle is assumed to shift lane if the vehicle's rear is

$\%$ ahead of the beginning of the gap and the time gap is acceptable

if $\quad$ isempty(iGap) \&\& locGapBegin2(iGap) $<=($ vehLocR(lagVeh)-

vehLenR(lagVeh)) \&\& availTotTimeGaps(iGap) $>=$ acceptMergeGapLn2

coopMerge $=0$;

cmMergeRin(lagVehRin) = analTime;

[vehLocR，headwaysR， critHeadwaysR， speedsR， decRatesR，vehLenR, sdRequest, cmMerge, ...

vehLoc2, headways2, critHeadways2, speeds2, decRates2, vehLen2] = LaneChange_v2(lagVeh, minF2lHeadway, ...

vehLocR, headwaysR, critHeadwaysR, speedsR, decRatesR, vehLenR, sdRequest, cmMerge, ...

vehLoc2, headways2, critHeadways2, speeds2, decRates2, vehLen2);

$\operatorname{vehIdx}(\operatorname{lagVeh})=[]$; 
end

end

if analTime $>=\operatorname{sclVehT} 1$

\%call function to calculate the gaps at SCL area

[availTotGaps, locGapBegin,

gapSpeeds]

TotalGap_v8(vehLocR,speedsR,vehLenR);

\%calculate time gaps and merge locations to the beginning and

$\%$ end of gap (beginning is closest to gore) and middle (gap

$\%$ divided 40/60)

for $\mathrm{i}=1$ :length (gapSpeeds)

nGaps = length(availTotGaps);

availTotTimeGaps = availTotGaps./gapSpeeds(i);

end

dist2Merge $=$ zeros(3, nGaps); $\% 3$ rows correspond to begin, mid, and end dist2Merge $(1,:)=$ sclRampCurveToGore + locGapBegin + sclVehLen; dist2Merge $(2,:) \quad=\quad$ sclRampCurveToGore + locGapBegin + availTotGaps*ratioLagGap + sclVehLen*(1-ratioLagGap); dist2Merge $(3,:)=$ sclRampCurveToGore + locGapBegin + availTotGaps;

relMergeLoc $=$ dist2Merge $/$ sclLength;

$\%$ check that there are no -ve values in time gaps and merge

\%locations

if any(availTotTimeGaps) $<0$

error('At least one time gaps is negative at SCL Veh \%d', iSCLVeh); end

time2Merge $=$ analTime - simTime;

\%Calculating the PNC of SCL vehicle for each available total gap in FRL

\%using cummulative distribution function

$\% \mathrm{PNC}$ is determined at beginning, middle, and end of gap

$\% \mathrm{PNC}$ for acceleration

sclVehAccRate $=2 *($ dist2Merge/time2Merge - goreSpeed $) /$ time2Merge; accFailurePNC $=$ normcdf( $\operatorname{sclVehAccRate,meanAccRate,sdAccRate);~}$ 
$\% \mathrm{SCL}$ vehicles should not be required to decelerate to merge

accFailurePNC $(\operatorname{sclVehAccRate}<0)=1$;

$\% \mathrm{PNC}$ for gap

$\%$ mean total gap equation 10

meanAcceptTotalGap $\quad=9.563 \quad-\quad\left(0.216^{*}\right.$ meanMergeSpeed $)$

(1.322*relMergeLoc);\%equation 5.14

minAcceptTotGap = sclVehLen./gapSpeeds + minFrlHeadway;

gapFailurePNC

normcdf(availTotTimeGaps,meanAcceptTotalGap,sdAcceptTotGap,'upper');

gapFailurePNC(availTotTimeGaps $<$ minAcceptTotGap $)=1$;

gapFailurePNC(availTotTimeGaps $>=$ maxReqGap) $=0$;

$\%$ combinedPNC due to total gap failure and acceleration failure

combinedPNC $=$ gapFailurePNC + accFailurePNC

(gapFailurePNC.*accFailurePNC);\%equation 5.15

finalCombinedPNC $=\min ($ finalCombinedPNC, $\min ($ combinedPNC(:)) ;

end

if analMethod $==3$

lnChangVeh = find(abs (cmMerge-analTime $)<$ timeStep/2,1);

if $\sim$ isempty (lnChangVeh)

$\%$ one vehicle will reach the time to change lane in the

$\%$ next time step

[vehLocR， headwaysR， critHeadwaysR， speedsR， decRatesR， vehLenR, sdRequest, cmMerge, ...

vehLoc2, headways2, critHeadways2, speeds2, decRates2, vehLen2] = LaneChange_v2(lnChangVeh, minF2lHeadway, ...

vehLocR, headwaysR, critHeadwaysR， speedsR， decRatesR，vehLenR, sdRequest, cmMerge, ...

vehLoc2, headways2, critHeadways2, speeds2, decRates2, vehLen2);

if $\sim$ isempty (lagVeh) \&\& lnChangVeh $<$ lagVeh

$\%$ a vehicle in front of the lag vehicle changed lane.

lagVeh = lagVeh - 1;

end

$\operatorname{vehIdx}(\operatorname{lnChangVeh})=[]$;

end

end 
end

\%check if PNC is to be recalculated while accounting for coopMerge

if analysisRun $==1$

if analMethod $>1 \& \&$ finalCombinedPNC1 $<=$ finalCombinedPNC

$\%$ coop merge was tried but did not improve PNC

\%cancel the coop merge and keep PNC according to method 1

finalCombinedPNC $=$ finalCombinedPNC1;

if analMethod $==2$

$\operatorname{sdRequest}(\operatorname{lagVeh})=0$;

else

cmMergeRin $(\operatorname{lagVehRin})=0 ; \%$ this is the case of analMethod $=3$ end

end

return

elseif coopMerge $==0 \|$ finalCombinedPNC $==0$

$\%$ coopMerge is not recommended or not needed

$\operatorname{cmMergeRin}(\operatorname{lagVehRin})=0 ; \%$ this is the case of analMethod $=3$

return

else

analysisRun $=1$;

vehLocR = vehLocRin;

headwaysR = headwaysRin;

critHeadwaysR = critHeadwaysRin;

speedsR = speedsRin;

sdRequest $=$ sdRequestRin;

cmMerge $=$ cmMergeRin;

finalCombinedPNC1 $=$ finalCombinedPNC; $\% \mathrm{PNC}$ using method 1

if analMethod $==2$

sdRequest $($ lagVeh $)=$ maxSlowReq*speedsR(lagVeh $)$;

else

decRatesR = decRatesRin;

vehLenR = vehLenRin;

vehLoc2 = vehLoc 2 in;

headways 2 = headways 2 in;

critHeadways 2 = critHeadways 2 in;

speeds $2=$ speeds 2 in;

decRates 2 = decRates2in;

vehLen2 = vehLen2in;

end

end

end 